\title{
Catalytic $\alpha$-Hydroarylation of Acrylates and Acrylamides via an Interrupted Hydro-Dehalogenation Reaction
}

\author{
Alena M. Vasquez†, John A. Gurak, Jr.†, Candice L. Joeł, Emily C. Cherney $\S$, Keary M. \\ Engle†* \\ $\dagger$ Department of Chemistry, The Scripps Research Institute, 10550 North Torrey Pines Road, La Jolla, California \\ 92037, United States \\ †Chemistry Development, Bristol-Myers Squibb, 1 Squibb Drive, New Brunswick, New Jersey 08903, United \\ States \\ $\S$ Discovery Chemistry, Bristol Myers Squibb, US Rt. 206 \& Province Line Road, Princeton, New Jersey 08540, \\ United States
}

\section{Table of Contents}

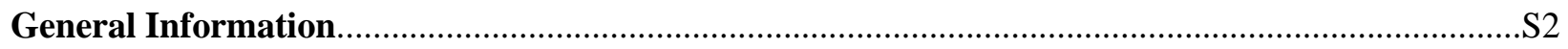

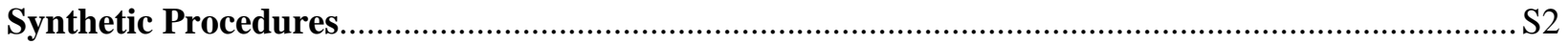

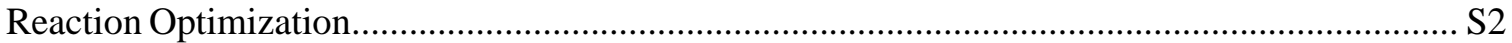

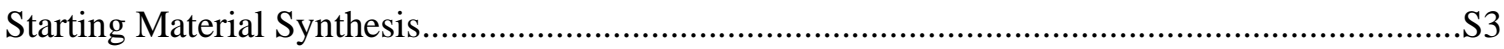

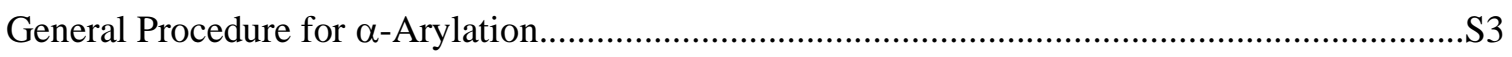

Characterization Data for $\alpha$-Arylated Products...................................................................... 5

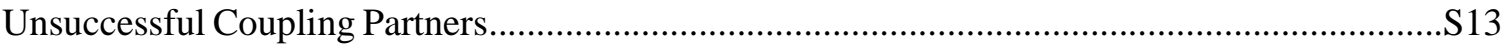

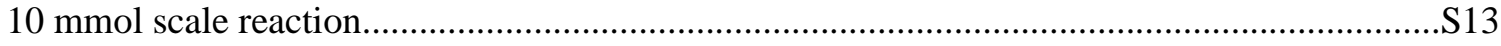

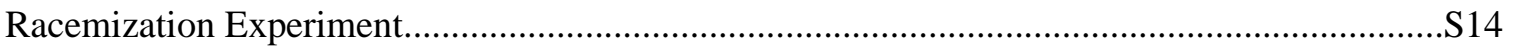

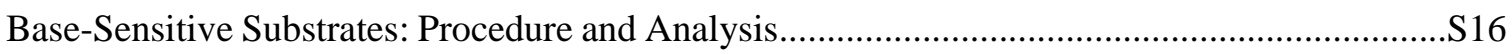

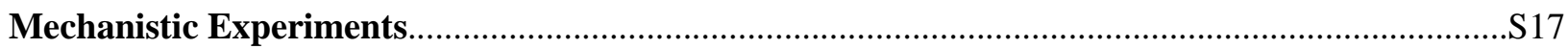

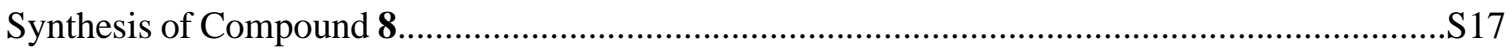

General Procedure for Stoichiometric Study ......................................................................... 17

General Procedure for Determination of Kinetic Isotope Effect................................................S17

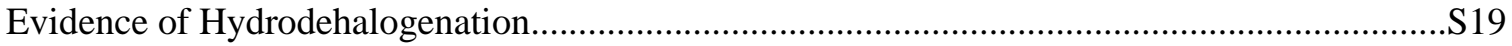

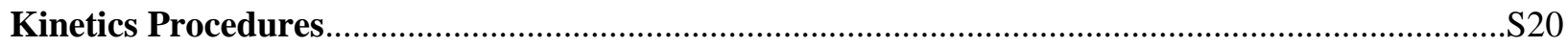

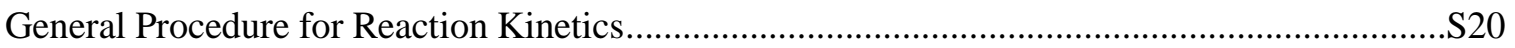

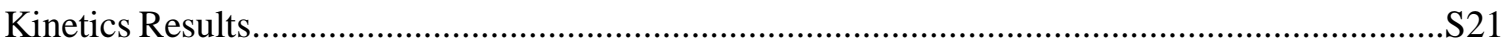

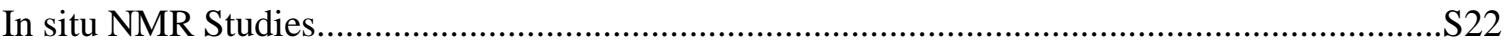

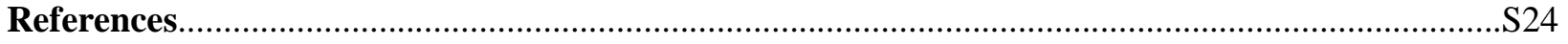

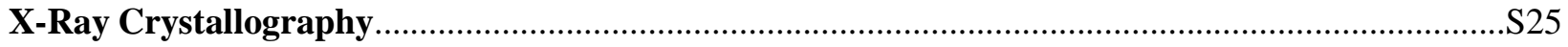

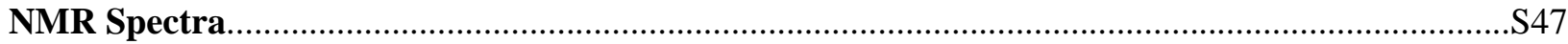




\section{GENERAL INFORMATION}

Unless otherwise noted, all materials were used as received from commercial sources without further purification. All chemicals were purchased from Aldrich, Alfa Aesar, Oakwood, and Combi-Blocks. ${ }^{1} \mathrm{H}$ and ${ }^{13} \mathrm{C}$ spectra were recorded on Bruker DRX-500 and AV-600 instruments. Spectra were internally referenced to $\mathrm{SiMe}_{4}$ or solvent signals. The following abbreviations (or combinations thereof) were used to explain multiplicities: $\mathrm{b}=$ broad, $\mathrm{s}=$ singlet, $\mathrm{d}=$ doublet, $\mathrm{t}=$ triplet, $\mathrm{q}=$ quartet, $\mathrm{p}=$ pentet, $\mathrm{m}=$ multiplet. High-resolution mass spectra (HRMS) for new compounds were recorded on an Agilent LC/MSD TOF mass spectrometer.

\section{SYNTHETIC PROCEDURES}

\section{Reaction Optimization}<smiles>C=CC(=O)N1CCOCC1</smiles><smiles>CC(C(=O)N1CCOCC1)c1ccccc1</smiles>

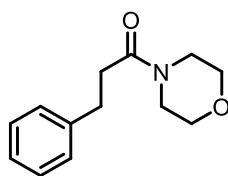

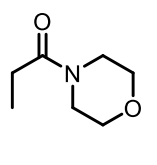

C

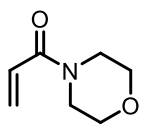

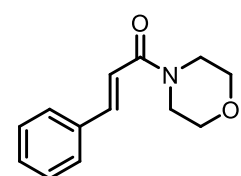

E

Table S1. Optimization of reaction conditions. ${ }^{\mathrm{a}}$

\begin{tabular}{|c|c|c|c|c|c|c|c|}
\hline Entry & Deviation from Written Conditions & $\boldsymbol{A}$ & $\boldsymbol{B}$ & $C$ & $D$ & $\boldsymbol{E}$ & $A: B$ \\
\hline 1 & None & $89 \%$ & $4 \%$ & $6 \%$ & $0 \%$ & $2 \%$ & $22: 1$ \\
\hline 2 & $0.5 \% \mathrm{Pd}_{2}(\mathrm{dba})_{3} / 5 \% \mathrm{P}\left(4-\mathrm{F}-\mathrm{C}_{6} \mathrm{H}_{4}\right)_{3}$ & $38 \%$ & $2 \%$ & $3 \%$ & $13 \%$ & $18 \%$ & 14:1 \\
\hline 3 & $1.0 \% \mathrm{Pd}_{2}(\mathrm{dba})_{3} / 5 \% \mathrm{P}(4-\mathrm{F}-\mathrm{Ph})_{3}$ & $11 \%$ & $1 \%$ & $1 \%$ & $11 \%$ & $55 \%$ & $11: 1$ \\
\hline 4 & $2.5 \% \mathrm{Pd}_{2}(\mathrm{dba})_{3} / 10 \% \mathrm{P}(4-\mathrm{F}-\mathrm{Ph})_{3}$ & $59 \%$ & $3 \%$ & $5 \%$ & $3 \%$ & $0 \%$ & 20:1 \\
\hline 5 & No ligand & $0 \%$ & $0 \%$ & $0 \%$ & $64 \%$ & $18 \%$ & - \\
\hline 6 & $\mathrm{PPh}_{3}$ instead of $\mathrm{P}(4-\mathrm{F}-\mathrm{Ph})_{3}$ & $62 \%$ & $5 \%$ & $6 \%$ & $0 \%$ & $2 \%$ & $12: 1$ \\
\hline 7 & $\mathrm{P}(3-\mathrm{Me}-\mathrm{Ph})_{3}$ instead of $\mathrm{P}(4-\mathrm{F}-\mathrm{Ph})_{3}$ & $85 \%$ & $7 \%$ & $11 \%$ & $0 \%$ & $0 \%$ & 11:1 \\
\hline 8 & $\mathrm{P}(4-\mathrm{OMe}-\mathrm{Ph})_{3}$ instead of $\mathrm{P}(4-\mathrm{F}-\mathrm{Ph})_{3}$ & $42 \%$ & $5 \%$ & $22 \%$ & $6 \%$ & $0 \%$ & $8: 1$ \\
\hline 9 & $\mathrm{P}\left(4-\mathrm{CF}_{3}-\mathrm{Ph}\right)_{3}$ instead of $\mathrm{P}(4-\mathrm{F}-\mathrm{Ph})_{3}$ & $36 \%$ & $2 \%$ & $1 \%$ & $25 \%$ & $5 \%$ & 18:1 \\
\hline 10 & $\mathrm{P}(3-\mathrm{Cl}-\mathrm{Ph})_{3}$ instead of $\mathrm{P}(4-\mathrm{F}-\mathrm{Ph})_{3}$ & $70 \%$ & $3 \%$ & $2 \%$ & $26 \%$ & $0 \%$ & 23:1 \\
\hline 11 & rac-binap instead of $\mathrm{P}(4-\mathrm{F}-\mathrm{Ph})_{3}$ & $36 \%$ & $4 \%$ & $10 \%$ & $36 \%$ & $0 \%$ & $15: 1$ \\
\hline 12 & dppe instead of $\mathrm{P}(4-\mathrm{F}-\mathrm{Ph})_{3}$ & $33 \%$ & $4 \%$ & $42 \%$ & $15 \%$ & $0 \%$ & $8: 1$ \\
\hline 13 & $\begin{array}{c}\mathrm{KHCO}_{2}+\mathrm{TBABF}_{4}(20 \%)+\mathrm{H}_{2} \mathrm{O}(30 \text { equiv. }) \\
\text { instead of TMA } \bullet \mathrm{HCO}_{2}\end{array}$ & $31 \%$ & $3 \%$ & $4 \%$ & $41 \%$ & $4 \%$ & $10: 1$ \\
\hline 14 & $\begin{array}{c}\mathrm{NaHCO}_{2}+\mathrm{TBABF}_{4}(20 \%)+\mathrm{H}_{2} \mathrm{O}(30 \text { equiv. }) \\
\text { instead of TMA } \bullet \mathrm{HCO}_{2}\end{array}$ & $29 \%$ & $2 \%$ & $6 \%$ & $45 \%$ & $5 \%$ & $15: 1$ \\
\hline 15 & $\begin{array}{c}\mathrm{NH}_{4} \mathrm{HCO}_{2}+\mathrm{TBABF}_{4}(20 \%)+\mathrm{H}_{2} \mathrm{O} \text { (30 equiv.) } \\
\text { instead of TMA } \bullet \mathrm{HCO}_{2}\end{array}$ & $59 \%$ & $3 \%$ & $4 \%$ & $9 \%$ & $0 \%$ & $20: 1$ \\
\hline 16 & $\mathrm{KOH}$ instead of $\mathrm{K}_{3} \mathrm{PO}_{4}$ & $14 \%$ & $24 \%$ & $4 \%$ & $0 \%$ & $0 \%$ & $1: 2$ \\
\hline 17 & $\mathrm{~K}_{2} \mathrm{CO}_{3}$ instead of $\mathrm{K}_{3} \mathrm{PO}_{4}$ & $67 \%$ & $4 \%$ & $8 \%$ & $0 \%$ & $4 \%$ & $17: 1$ \\
\hline 18 & DCM instead of 1,4-dioxane & $38 \%$ & $10 \%$ & $8 \%$ & $32 \%$ & $5 \%$ & $4: 1$ \\
\hline 19 & DMF instead of 1,4-dioxane & $63 \%$ & $3 \%$ & $11 \%$ & $4 \%$ & $6 \%$ & 21:1 \\
\hline 20 & $\mathrm{No} \mathrm{Pd}_{2}(\mathrm{dba})_{3}$ & $0 \%$ & $0 \%$ & $0 \%$ & $80 \%$ & $0 \%$ & - \\
\hline 21 & $\mathrm{No} \mathrm{TMA} \bullet \mathrm{HCO}_{2}$ & $0 \%$ & $0 \%$ & $0 \%$ & $90 \%$ & $4 \%$ & - \\
\hline 22 & No TMAHCO 2,30 equiv. $\mathrm{H}_{2} \mathrm{O}$ & 0 & 0 & $8 \%$ & $0 \%$ & $76 \%$ & - \\
\hline
\end{tabular}

${ }^{a}$ Reactions were performed on $0.05 \mathrm{mmol}$ scale following general procedure A. 


\section{Starting Material Synthesis}

Substrates $4 \mathbf{d},{ }^{1} 4 \mathbf{e},{ }^{1} 4 \mathbf{h},{ }^{2} 4 \mathbf{i},{ }^{2} 4 \mathbf{j},{ }^{3} 4 \mathbf{m},{ }^{4} 4 \mathbf{n},{ }^{5} 4 \mathbf{c o},{ }^{5} 4 \mathbf{p},{ }^{6} 4 \mathbf{q},{ }^{7} 4 \mathbf{r}^{10} 4 \mathbf{s},{ }^{11}$ and $9^{12}$ were prepared according to literature procedures. Substrates 1a, $\mathbf{4 a}, \mathbf{4 b}, \mathbf{4 c}, \mathbf{4 f}, \mathbf{4 k}, \mathbf{4 l}$, and $\mathbf{2 a - q}$ were purchased from MilliporeSigma.

Scheme S1: Synthesis of $\mathbf{4 g}$

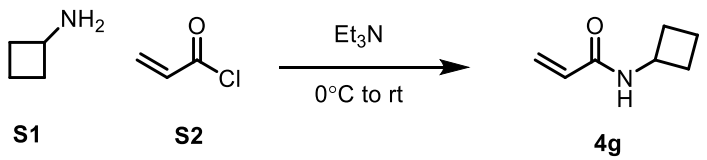

To a stirred solution of cyclobutylamine $(213 \mathrm{mg}, 3.00 \mathrm{mmol})$ in DCM $(10 \mathrm{~mL})$ was added triethylamine $(911 \mathrm{mg}, 1.2 \mathrm{~mL})$ and acroyl chloride $(272 \mathrm{mg}, 3.00 \mathrm{mmol})$ dropwise. The solution was allowed to warm to room temperature overnight. Sodium bicarbonate was added, and the aqueous solution was extracted with $\mathrm{Et}_{2} \mathrm{O}(10 \mathrm{~mL} \times 3)$. The organic layer was dried over $\mathrm{MgSO}_{4}$, filtered, and concentrated. The residue was purified by flash column chromatography to yield $\mathbf{4 g}$ as a white solid (330 $\mathrm{mg}, 89 \%)$. ${ }^{1} \mathbf{H}$ NMR (600 $\left.\mathrm{MHz}, \mathrm{CDCl}_{3}\right) \delta 6.29(\mathrm{dd}, J=17.0,1.4 \mathrm{~Hz}, 1 \mathrm{H}), 6.11-6.03(\mathrm{~m}, 1 \mathrm{H}), 5.72($ broad s, $1 \mathrm{H}) 5.65(\mathrm{dd}, J=10.3$, $1.4 \mathrm{~Hz}, 1 \mathrm{H}), 4.55-4.47(\mathrm{~m}, 1 \mathrm{H}), 2.40$ (tddd, $J=8.7,3.9,3.2,2.1 \mathrm{~Hz}, 2 \mathrm{H}), 1.96-1.86(\mathrm{~m}, 2 \mathrm{H}), 1.80-1.70$ $(\mathrm{m}, 2 \mathrm{H}) .{ }^{13} \mathrm{C} \mathrm{NMR}\left(150 \mathrm{MHz}, \mathrm{CDCl}_{3}\right) \delta 164.00,130.39,125.98,44.26,30.82,14.69$.

\section{General Procedure for $\alpha$-Arylation}

Preparation of Pd-Ligand Stock Solution:

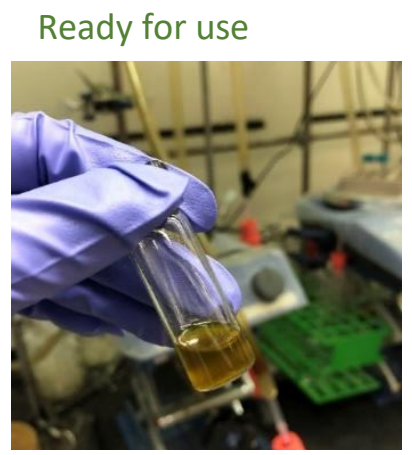

\section{Unsuitable for use}

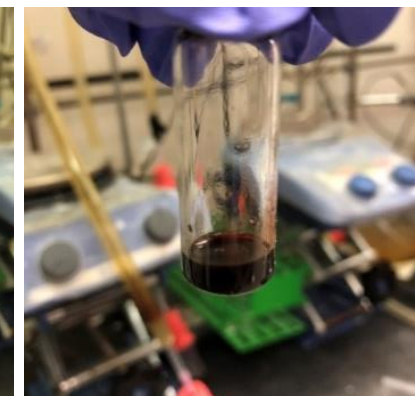

To a one-dram vial containing a stir bar were added $\mathrm{Pd}_{2}(\mathrm{dba})_{3}(45.8 \mathrm{mg}, 50 \mu \mathrm{mol}$ for $\mathrm{A} ; 18.3 \mathrm{mg}, 20$ $\mu \mathrm{mol}$ for B), tris(4-fluorophenyl)phosphine (127 mg, $0.400 \mathrm{mmol}$ for A; $31.6 \mathrm{mg}, 0.100 \mathrm{mmol}$ for B), and dioxane $(1.33 \mathrm{~mL})$. The suspension was capped and allowed to stir until all solids had dissolved (around $5 \mathrm{~min})$. An aliquot $(133 \mu \mathrm{L})$ was then transferred into the reaction vial.

Notes:

Figure S1. Pd-ligand stock solution freshly made and used immediately for each experiment.

- When solvent is initially added, the solution will appear dark red in color and will become brown/dark orange in color after stirring for a few minutes, at which point it should be used.

- If the solution changes color and becomes red again, it is no longer suitable for use.

- For a recommended workflow, we suggest first preparing the stock solution, and allowing it to mix while measuring the other liquid reaction components (aryl iodide, acrylamide formate). Upon completion of these operations, the stock solution is typically ready for use. 


\section{General Procedure A:}

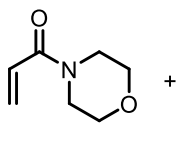

1a

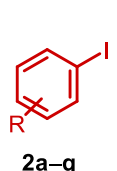

$2 a-q$

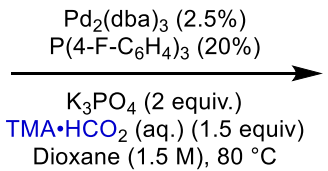

Dioxane $(1.5 \mathrm{M}), 80^{\circ} \mathrm{C}$

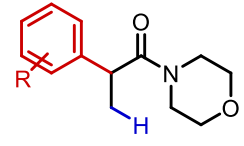

$3 \mathbf{a}-\mathbf{q}$
$\alpha: \beta$

Scheme S2. Gernal Procedure A

To a one-dram vial containing a stir bar was added $\mathrm{K}_{3} \mathrm{PO}_{4}(85 \mathrm{mg}, 0.40 \mathrm{mmol})$. Using a micropipette, aryl iodide $(0.240 \mathrm{mmol})$, 4-acryloylmorpholine $(28.2 \mathrm{mg}, 25.2 \mu \mathrm{L}, 0.200 \mathrm{mmol})$, tetramethylammonium formate $(30 \mathrm{wt} \%, 100 \mu \mathrm{L}, 0.290 \mathrm{mmol})$, and $\mathrm{Pd} / \mathrm{Ligand}$ stock solution $(133 \mu \mathrm{L})$ were added sequentially to the vial. The vial was sealed and placed in a pre-heated heating block at $80{ }^{\circ} \mathrm{C}$ for $4 \mathrm{~h}$. After $4 \mathrm{~h}$, the vial was cooled to room temperature. A small aliquot was removed and dissolved in $\mathrm{CDCl}_{3}$ for ${ }^{1} \mathrm{H} \mathrm{NMR}$ analysis $(600 \mathrm{MHz})$. Regioisomeric ratios $(\alpha: \beta)$ were determined by comparing the average integration of the $\beta$ $\mathrm{CH}_{2}$ triplets $(\sim 2.5-3 \mathrm{ppm})$ to the integration of methyl $\mathrm{CH}_{3}$ doublet $(\sim 1.5 \mathrm{ppm})$. Percentage yields represent the isolated yields of pure $\alpha$-arylated product unless otherwise specified.

General Procedure B:

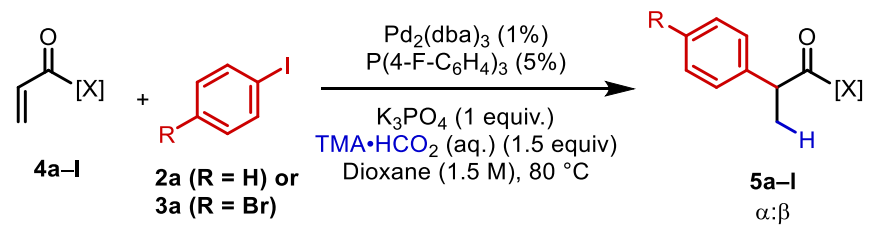

Scheme S3. General Procedure B

To a one-dram vial containing a stir bar was added $\mathrm{K}_{3} \mathrm{PO}_{4}(43 \mathrm{mg}, 0.2 \mathrm{mmol})$. Using a micropipette, $\mathrm{PhI}$ (49.0 mg, $26.9 \mu \mathrm{L}, 0.240 \mathrm{mmol})$, acrylate/acrylamide $(0.200 \mathrm{mmol})$, tetramethylammonium formate (30 $\mathrm{wt} \%, 100 \mu \mathrm{L}, 0.290 \mathrm{mmol})$, and Pd/Ligand stock solution $(133 \mu \mathrm{L})$ were added sequentially to the vial. The vial was sealed and placed in a pre-heated heating block at $80^{\circ} \mathrm{C}$ for $4 \mathrm{~h}$. After $4 \mathrm{~h}$ the vial was cooled to room temperature. A small aliquot was removed and dissolved in $\mathrm{CDCl}_{3}$ for ${ }^{1} \mathrm{H} \mathrm{NMR}$ analysis $(600 \mathrm{MHz})$. Regioisomeric ratios $(\alpha: \beta)$ were determined by comparing the average integration of the $\beta-\mathrm{CH}_{2}$ triplets $(\sim 2.5-3 \mathrm{ppm})$ to the integration of methyl $\mathrm{CH}_{3}$ doublet $(\sim 1.5 \mathrm{ppm})$. The NMR sample was recombined with the reaction mixture, and the solution was loaded directly onto a preparative TLC plate (40\% EtOAc/Hexanes). Percentage yields represent the isolated yields of pure $\alpha$-arylated product unless otherwise specified.

General Procedure C:

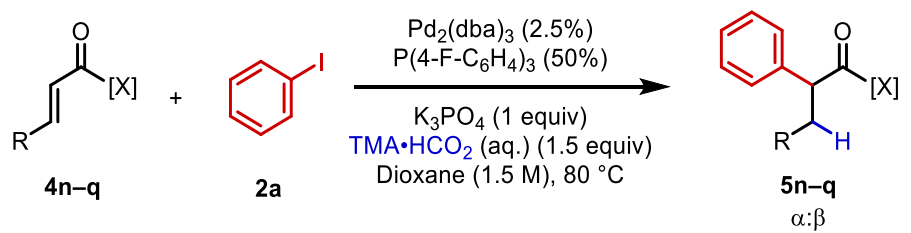

Scheme S4. Gernal Procedure C

To a one-dram vial containing a stir bar was added $\mathrm{K}_{3} \mathrm{PO}_{4}(85 \mathrm{mg}, 0.40 \mathrm{mmol})$. Using a micropipette, aryl iodide $(0.240 \mathrm{mmol})$, acrylamide $(0.200 \mathrm{mmol})$, tetramethylammonium formate $(30 \mathrm{wt} \%, 100 \mu \mathrm{L}, 0.290$ 
$\mathrm{mmol})$, and $\mathrm{Pd} /$ Ligand stock solution $(133 \mu \mathrm{L})$ were added sequentially to the vial. The vial was sealed and placed in a pre-heated heating block at $100{ }^{\circ} \mathrm{C}$ for $16 \mathrm{~h}$. After $16 \mathrm{~h}$ the vial was cooled to room temperature. A small aliquot was removed and dissolved in $\mathrm{CDCl}_{3}$ for ${ }^{1} \mathrm{H} \mathrm{NMR}$ analysis (600 $\mathrm{MHz}$ ). The NMR sample was recombined with the reaction mixture, and the sample was purified by prep LCMS. Percentage yields represent the isolated yields of pure $\alpha$-arylated product unless otherwise specified.

$\underline{\text { Representative }{ }^{1} \mathrm{H} \text { NMR Spectrum of Crude Reaction Mixture: }}$<smiles>COc1ccc(I)cc1</smiles><smiles>C=CC(=O)N1CCOCC1</smiles>

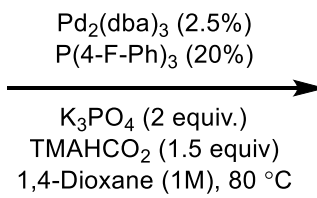<smiles>COc1ccc(CCC(=O)N2CCOCC2)cc1</smiles>

A

B<smiles>CCC(=O)N1CCOCC1</smiles>

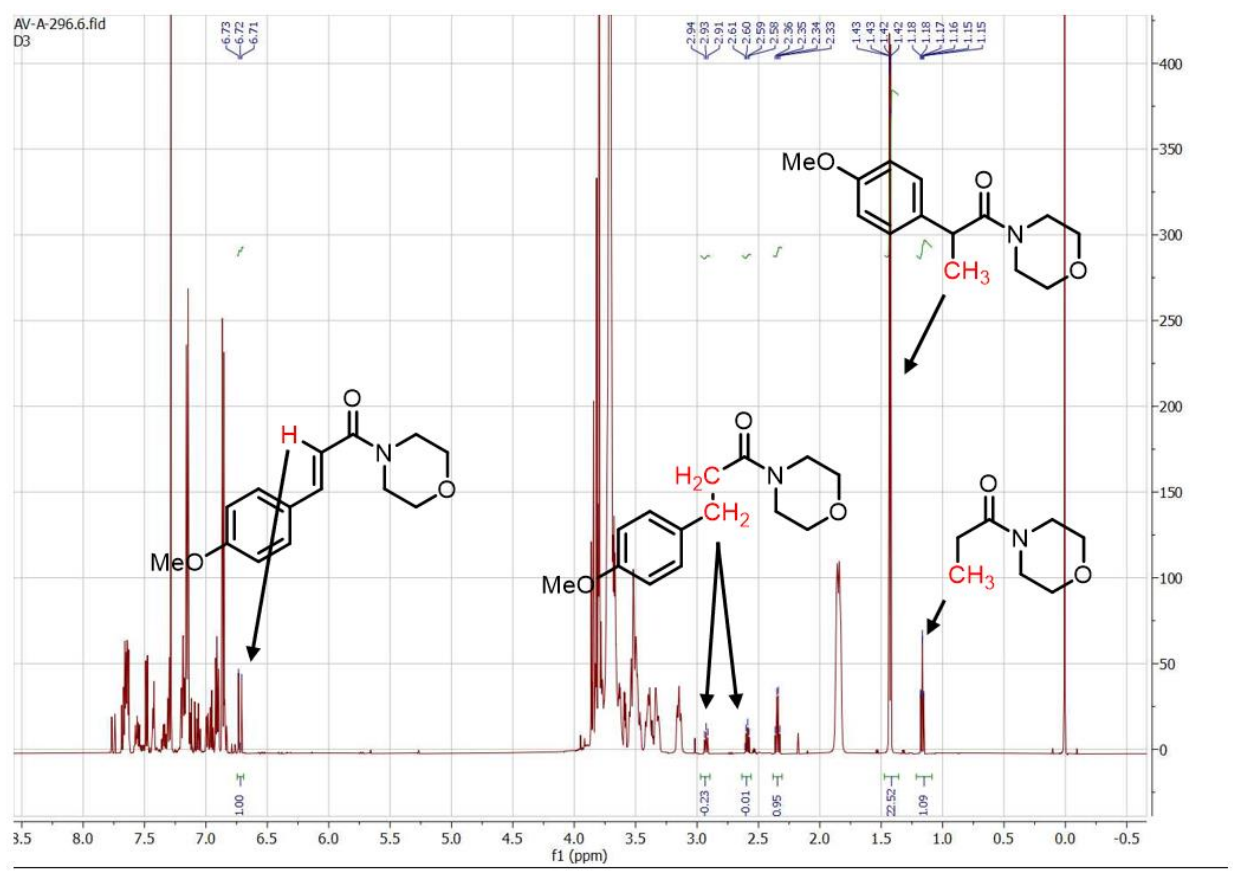

Figure S2. Representative NMR spectra.

\section{Characterization Data for $\alpha$-Arylated Products:}

1-morpholino-2-phenylpropan-1-one (3a): The title compound was prepared on a $0.200 \mathrm{mmol}$ scale<smiles>CC(C(=O)N1CCOCC1)c1ccccc1</smiles>
according to General Procedure A. The crude residue was purified by preparative TLC (40\% EtOAc/hexanes). Purification afforded 3a as an off-white solid (39.6 mg, 90\%). Analytical data was in agreement with literature values. ${ }^{14}{ }^{1} \mathbf{H}$ NMR $\left(600 \mathrm{MHz}, \mathrm{CDCl}_{3}\right) \delta$ ${ }^{1} \mathrm{H}$ NMR $\left(600 \mathrm{MHz}, \mathrm{CDCl}_{3}\right) \delta 7.37-7.31(\mathrm{~m}, 2 \mathrm{H}), 7.28-7.23(\mathrm{~m}, 3 \mathrm{H}), 3.89-3.78(\mathrm{~m}$, 2H), 3.69 (ddd, $J=13.6,9.4,5.2 \mathrm{~Hz}, 1 \mathrm{H}), 3.59-3.53(\mathrm{~m}, 2 \mathrm{H}), 3.53-3.47(\mathrm{~m}, 2 \mathrm{H}), 3.45$ - $3.38(\mathrm{~m}, 1 \mathrm{H}), 3.36-3.29(\mathrm{~m}, 1 \mathrm{H}), 3.13(\mathrm{ddd}, J=11.1,7.4,3.0 \mathrm{~Hz}, 1 \mathrm{H}), 1.48(\mathrm{~d}, J=6.9 \mathrm{~Hz}, 3 \mathrm{H}) .{ }^{13} \mathbf{C}$ NMR $\left(150 \mathrm{MHz}, \mathrm{CDCl}_{3}\right) \delta 171.71,141.39,128.55,126.69,126.44,66.33,65.83,45.54,42.81,41.92$, 20.17. HRMS calcd. for $\mathrm{C}_{13} \mathrm{H}_{17} \mathrm{NO}_{2}{ }^{+}[\mathrm{M}+\mathrm{H}]^{+}:$220.1338, Found: 220.1337 . 
1-morpholino-2-(4-(trifluoromethyl)phenyl)propan-1-one (3b): The title compound was prepared on a

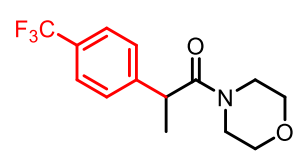

$0.200 \mathrm{mmol}$ scale according to General Procedure A. The crude residue was purified by preparative TLC (40\% EtOAc/hexanes). Purification afforded $\mathbf{3 b}$ as an off-white solid $(49.7 \mathrm{mg}, 87 \%)$. Analytical data was in agreement with literature values. ${ }^{15} \mathbf{H}$ NMR $\left(600 \mathrm{MHz}, \mathrm{CDCl}_{3}\right) \delta 7.61(\mathrm{~d}, J=8.1 \mathrm{~Hz}, 2 \mathrm{H}), 7.40(\mathrm{~d}, J=8.0 \mathrm{~Hz}, 2 \mathrm{H}), 3.95(\mathrm{q}$, $J=6.9 \mathrm{~Hz}, 1 \mathrm{H}), 3.80-3.63(\mathrm{~m}, 2 \mathrm{H}), 3.58(\mathrm{~h}, J=8.0 \mathrm{~Hz}, 3 \mathrm{H}), 3.50-3.36(\mathrm{~m}, 1 \mathrm{H}), 3.37-$ $3.17(\mathrm{~m}, 2 \mathrm{H}), 1.49(\mathrm{~d}, J=6.9 \mathrm{~Hz}, 3 \mathrm{H}) .{ }^{13} \mathbf{C} \mathbf{N M R}\left(150 \mathrm{MHz}, \mathrm{CDCl}_{3}\right) \delta 171.42,145.74$, $129.37\left(\mathrm{q}, J_{C-F}=35 \mathrm{~Hz}\right), 127.61,125.99\left(\mathrm{q}, J_{C-F}=2.8 \mathrm{~Hz}\right), 123.96\left(\mathrm{q}, J_{C-F}=274 \mathrm{~Hz}\right), 66.32,65.85,45.73$, 42.40, 41.97, 20.01. ${ }^{19} \mathbf{F}$ NMR $\left(376 \mathrm{MHz}, \mathrm{CDCl}_{3}\right) \delta-62.8$ HRMS calcd. for $\mathrm{C}_{14} \mathrm{H}_{16} \mathrm{~F}_{3} \mathrm{NO}_{2}{ }^{+}[\mathrm{M}+\mathrm{H}]^{+}$: 288.1211, Found: 288.1208.

2-(4-acetylphenyl)-1-morpholinopropan-1-one (3c): The title compound was prepared on a $0.200 \mathrm{mmol}$

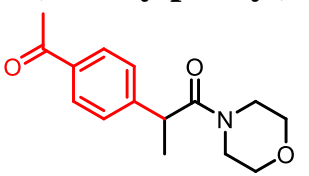

$3 c$ scale according to General Procedure A. The crude residue was purified by preparative TLC (40\% EtOAc/hexanes). Purification afforded $\mathbf{3 c}$ as an off-white solid (40.8 mg, 78\%). ${ }^{1} \mathbf{H}$ NMR $\left(600 \mathrm{MHz}, \mathrm{CDCl}_{3}\right) \delta$ 7.98-7.93 (m, 2H), 7.40-7.34 (m, $2 \mathrm{H}), 3.94(\mathrm{q}, J=6.9 \mathrm{~Hz}, 1 \mathrm{H}), 3.82-3.76(\mathrm{~m}, 1 \mathrm{H}), 3.70(\mathrm{t}, J=7.5 \mathrm{~Hz}, 1 \mathrm{H}), 3.55(\mathrm{p}, J$ $=9.0,8.3 \mathrm{~Hz}, 3 \mathrm{H}), 3.43(\mathrm{dd}, J=13.4,7.6 \mathrm{~Hz}, 1 \mathrm{H}), 3.29(\mathrm{~d}, J=13.4 \mathrm{~Hz}, 1 \mathrm{H}), 3.18(\mathrm{t}$, $J=10.1 \mathrm{~Hz}, 1 \mathrm{H}), 2.62(\mathrm{~s}, 3 \mathrm{H}), 1.50(\mathrm{~d}, J=6.9 \mathrm{~Hz}, 3 \mathrm{H}) .{ }^{13} \mathbf{C ~ N M R}\left(150 \mathrm{MHz}, \mathrm{CDCl}_{3}\right)$ $\delta 197.08,170.98,146.72,135.49,128.69,127.01,66.31,65.84,45.54,42.67,41.99,26.14,19.92$. HRMS calcd. for $\mathrm{C}_{15} \mathrm{H}_{19} \mathrm{NO}_{3}{ }^{+}[\mathrm{M}+\mathrm{H}]^{+}:$262.1443, Found: 262.1448 .

2-(4-bromophenyl)-1-morpholinopropan-1-one (3d): The title compound was prepared on a $0.200 \mathrm{mmol}$ scale according to General Procedure A. The crude residue was purified by preparative TLC (40\% EtOAc/hexanes). Purification afforded 3d as an off-white solid (55.6 mg, 93\%). ${ }^{1} \mathbf{H}$ NMR $\left(600 \mathrm{MHz}, \mathrm{CDCl}_{3}\right) \delta 7.44-7.39(\mathrm{~m}, 2 \mathrm{H}), 7.24-7.18(\mathrm{~m}, 2 \mathrm{H}), 3.84(\mathrm{q}$, $J=6.8 \mathrm{~Hz}, 1 \mathrm{H}), 3.80-3.75(\mathrm{~m}, 1 \mathrm{H}), 3.74-3.66(\mathrm{~m}, 1 \mathrm{H}), 3.57(\mathrm{~h}, J=8.3,7.6 \mathrm{~Hz}, 3 \mathrm{H})$, $3.47-3.40(\mathrm{~m}, 1 \mathrm{H}), 3.24(\mathrm{ddd}, J=10.6,7.1,2.9 \mathrm{~Hz}, 1 \mathrm{H}), 1.47(\mathrm{~d}, J=6.8 \mathrm{~Hz}, 3 \mathrm{H}) .{ }^{13} \mathbf{C}$ NMR $\left(150 \mathrm{MHz}, \mathrm{CDCl}_{3}\right) \delta 171.50,144.02,130.58,130.32,130.16,125.80,122.96,66.33,46.02,42.75$, 42.43, 20.53. HRMS calcd. for $\mathrm{C}_{13} \mathrm{H}_{16} \mathrm{BrNO}_{2}{ }^{+}[\mathrm{M}+\mathrm{H}]^{+}$: 298.0443, Found: 298.0439.

4-(1-morpholino-1-oxopropan-2-yl)benzaldehyde (3e): The title compound was prepared on a 0.200 $\mathrm{OHC}$

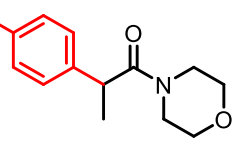

$3 e$ mmol scale according to General Procedure A. The crude residue was purified by preparative TLC (40\% EtOAc/hexanes). Purification afforded $3 \mathbf{e}$ as an off-white solid (32.2 mg, 65\%). ${ }^{1} \mathbf{H}$ NMR $\left(600 \mathrm{MHz}, \mathrm{CDCl}_{3}\right) \delta 10.01(\mathrm{~s}, 1 \mathrm{H}), 7.90-7.85(\mathrm{~m}$, 2H), 7.46-7.42 (m, 2H), $3.96(\mathrm{q}, J=6.8 \mathrm{~Hz}, 1 \mathrm{H}), 3.77(\mathrm{dt}, J=12.8,7.0 \mathrm{~Hz}, 1 \mathrm{H}), 3.69$ $(\mathrm{dd}, J=9.0,6.1 \mathrm{~Hz}, 1 \mathrm{H}), 3.55(\mathrm{t}, J=8.6 \mathrm{~Hz}, 3 \mathrm{H}), 3.43(\mathrm{ddd}, J=13.1,7.1,3.0 \mathrm{~Hz}$, $1 \mathrm{H}), 3.29$ (ddd, $J=13.4,5.8,2.9 \mathrm{~Hz}, 1 \mathrm{H}), 3.19$ (ddd, $J=10.8,7.2,2.9 \mathrm{~Hz}, 1 \mathrm{H}), 1.50$ (d, $J=6.9 \mathrm{~Hz}, 3 \mathrm{H})$. ${ }^{13} \mathbf{C ~ N M R}\left(150 \mathrm{MHz}, \mathrm{CDCl}_{3}\right) \delta 191.18,170.81,148.22,134.82,130.03,127.49,66.30,65.82,45.55,42.78$, 42.00, 19.89. HRMS calcd. for $\mathrm{C}_{14} \mathrm{H}_{17} \mathrm{NO}_{3}{ }^{+}[\mathrm{M}+\mathrm{H}]^{+}:$248.1287, Found: 248.1287.

1-morpholino-2-(p-tolyl)propan-1-one (3f): The title compound was prepared on a $0.200 \mathrm{mmol}$ scale Me according to General Procedure A. The crude residue was purified by preparative TLC (40\% EtOAc/hexanes). Purification afforded $\mathbf{3 f}$ as an off white solid. (38.8 $\mathrm{mg}, 83 \%)$. ${ }^{1} \mathbf{H}$ NMR $\left(600 \mathrm{MHz}, \mathrm{CDCl}_{3}\right) \delta 7.17-7.11(\mathrm{~m}, 4 \mathrm{H}), 3.81(\mathrm{dq}, J=12.7,6.8 \mathrm{~Hz}, 2 \mathrm{H})$, $3.71-3.66(\mathrm{~m}, 1 \mathrm{H}), 3.59-3.47(\mathrm{~m}, 3 \mathrm{H}), 3.41(\mathrm{ddd}, J=13.3,7.4,3.0 \mathrm{~Hz}, 1 \mathrm{H}), 3.36-$ 3f $\quad 3.29(\mathrm{~m}, 1 \mathrm{H}), 3.15(\mathrm{ddd}, J=11.2,7.4,3.2 \mathrm{~Hz}, 1 \mathrm{H}), 2.34(\mathrm{~s}, 3 \mathrm{H}), 1.45(\mathrm{~d}, J=6.8 \mathrm{~Hz}$, 3H). ${ }^{13} \mathrm{C}$ NMR $\left(150 \mathrm{MHz}, \mathrm{CDCl}_{3}\right) \delta 171.91,138.35,136.03,129.21,126.55,66.34,65.87,45.53,42.37$, 41.90, 20.56, 20.22. HRMS calcd. for $\mathrm{C}_{14} \mathrm{H}_{19} \mathrm{NO}_{2}{ }^{+}[\mathrm{M}+\mathrm{H}]^{+}: 234.1494$, Found: 234.1491. 
1-morpholino-2-(4-nitro-3-(trifluoromethyl)phenyl)propan-1-one (3g): The title compound was

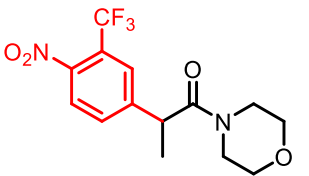

$3 \mathbf{g}$ prepared on an $0.200 \mathrm{mmol}$ scale according to General Procedure A. The crude residue was purified by preparative TLC (40\% EtOAc/hexanes). Purification afforded $3 \mathrm{~g}$ (containing 5\% tris(4-fluorophenyl)phosphine oxide) as an off white solid. (35.7 mg, 54\%). ${ }^{1} \mathbf{H}$ NMR $\left(600 \mathrm{MHz}, \mathrm{CDCl}_{3}\right){ }^{1} \mathrm{H}$ NMR $\left(600 \mathrm{MHz}, \mathrm{CDCl}_{3}\right) \delta 7.90(\mathrm{~d}, J=$ $8.3 \mathrm{~Hz}, 1 \mathrm{H}), 7.74(\mathrm{~d}, J=2.0 \mathrm{~Hz}, 1 \mathrm{H}), 7.68(\mathrm{dd}, J=8.3,2.0 \mathrm{~Hz}, 1 \mathrm{H}), 4.08(\mathrm{q}, J=6.9$ $\mathrm{Hz}, 1 \mathrm{H}), 3.68$ (tdd, $J=22.0,9.2,4.5 \mathrm{~Hz}, 5 \mathrm{H}), 3.53(\mathrm{ddd}, J=12.9,6.6,2.8 \mathrm{~Hz}, 1 \mathrm{H})$, 3.44 (ddd, $J=11.0,6.7,3.0 \mathrm{~Hz}, 1 \mathrm{H}), 3.36$ (ddd, $J=13.2,6.4,2.8 \mathrm{~Hz}, 1 \mathrm{H}), 1.54(\mathrm{~d}, J=6.9 \mathrm{~Hz}, 3 \mathrm{H}) .{ }^{13} \mathbf{C}$ NMR $\left(150 \mathrm{MHz}, \mathrm{CDCl}_{3}\right) \delta 170.45,147.20,146.88,132.00,127.11\left(\mathrm{q}, J_{C-F}=5.17 \mathrm{~Hz}\right), 125.76,124.29(\mathrm{q}$, $\left.J_{C-F}=33.93 \mathrm{~Hz}\right), 121.79\left(\mathrm{q}, J_{C-F}=275 \mathrm{~Hz}\right) 66.77,45,81,42.57,42.09,20.26 .{ }^{19} \mathbf{F ~ N M R}\left(564 \mathrm{MHz}, \mathrm{CDCl}_{3}\right)$ $\delta$-60.1193. HRMS calcd. for $\mathrm{C}_{14} \mathrm{H}_{15} \mathrm{~F}_{3} \mathrm{~N}_{2} \mathrm{O}_{4}{ }^{+}[\mathrm{M}+\mathrm{H}]^{+}: 333.1062$, Found: 333.1066 .

4-(1-morpholino-1-oxopropan-2-yl)-2-(trifluoromethyl)benzonitrile (3h): The title compound was

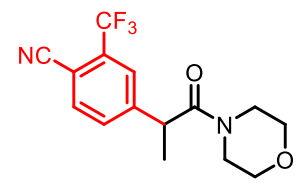

$3 \mathrm{~h}$

prepared on a $0.200 \mathrm{mmol}$ scale according to General Procedure A. The crude residue was purified by preparative TLC (40\% EtOAc/hexanes). Purification afforded $\mathbf{3 h}$ as an off white solid. (35.9 mg, 54\%). ${ }^{1} \mathbf{H}$ NMR $\left(600 \mathrm{MHz}, \mathrm{CDCl}_{3}\right) \delta 7.84(\mathrm{~d}, J=8.0 \mathrm{~Hz}$, $1 \mathrm{H}), 7.72(\mathrm{~d}, J=1.8 \mathrm{~Hz}, 1 \mathrm{H}), 7.65(\mathrm{dd}, J=8.0,1.8 \mathrm{~Hz}, 1 \mathrm{H}), 4.06(\mathrm{q}, J=6.9 \mathrm{~Hz}, 1 \mathrm{H})$, 3.74-3.61 (m, 5H), 3.55-3.49 (m, 1H), 3.45-3.38 (m, 1H), 3.38-3.31 (m, 1H), 1.54 $(\mathrm{d}, J=6.9 \mathrm{~Hz}, 3 \mathrm{H}) .{ }^{13} \mathbf{C} \mathbf{N M R}\left(150 \mathrm{MHz}, \mathrm{CDCl}_{3}\right) \delta 170.14,146.96,134.79,132.94$ $(\mathrm{q}, J=32.6 \mathrm{~Hz}), 130.85,125.51(\mathrm{q}, J=4.5 \mathrm{~Hz}), 121.75(\mathrm{~d}, J=274.0 \mathrm{~Hz}), 114.77,108.36,66.31,65.90$, 45.73, 42.21, 42.01, 19.68. ${ }^{19} \mathbf{F}$ NMR $\left(564 \mathrm{MHz}, \mathrm{CDCl}_{3}\right) \delta-62.08$. HRMS calcd. for $\mathrm{C}_{15} \mathrm{H}_{15} \mathrm{~F}_{3} \mathrm{~N}_{2} \mathrm{O}_{2}{ }^{+}$ $[\mathrm{M}+\mathrm{H}]^{+}:$313.1164, Found: 313.1165.

2-(benzo[d][1,3]dioxol-5-yl)-1-morpholinopropan-1-one (3i): The title compound was prepared on a

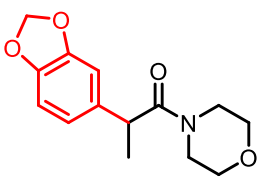

$3 \mathbf{i}$

$0.200 \mathrm{mmol}$ scale according to General Procedure A. The crude residue was purified by preparative TLC (60\% EtOAc/hexanes). Purification afforded $\mathbf{3 i}$ as an off-white solid $(30.3 \mathrm{mg}, 58 \%) .{ }^{1} \mathbf{H}$ NMR $\left(600 \mathrm{MHz}, \mathrm{CDCl}_{3}\right) \delta 6.79-6.74(\mathrm{~m}, 2 \mathrm{H}), 6.68(\mathrm{dd}, J=8.0$, $1.8 \mathrm{~Hz}, 1 \mathrm{H}), 5.96(\mathrm{~s}, 2 \mathrm{H}), 3.78(\mathrm{q}, J=6.7 \mathrm{~Hz}, 2 \mathrm{H}), 3.72-3.66(\mathrm{~m}, 1 \mathrm{H}), 3.55(\mathrm{dtdd}, J=$ 14.2, 10.7, 7.2, $2.7 \mathrm{~Hz}, 3 \mathrm{H}), 3.43$ (ddd, $J=13.2,7.0,2.8 \mathrm{~Hz}, 1 \mathrm{H}), 3.39-3.33(\mathrm{~m}, 1 \mathrm{H})$, 3.24 (ddd, $J=10.8,7.3,3.0 \mathrm{~Hz}, 1 \mathrm{H}), 1.43(\mathrm{~s}, 3 \mathrm{H}) .{ }^{13} \mathbf{C ~ N M R}\left(150 \mathrm{MHz}, \mathrm{CDCl}_{3}\right) 171.73$, 147.63, 145.97, 135.19, 119.80, 108.10, 107.10, 100.62, 66.35, 65.94, 45.55, 42.31, 20.28. HRMS calcd. for $\mathrm{C}_{14} \mathrm{H}_{17} \mathrm{NO}_{4}{ }^{+}[\mathrm{M}+\mathrm{H}]^{+}:$264.1236, Found: 264.1232 .

2-(3-acetylphenyl)-1-morpholinopropan-1-one (3j): The title compound was prepared on a $0.200 \mathrm{mmol}$

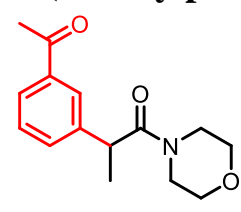

3j scale according to General Procedure A. The crude residue was purified by preparative TLC (40\% EtOAc/hexanes). Purification afforded $\mathbf{3 j}$ as an off-white solid. (47.2 $\mathrm{mg}$, 90\%). ${ }^{1} \mathbf{H}$ NMR $\left(600 \mathrm{MHz}, \mathrm{CDCl}_{3}\right) \delta 7.85(\mathrm{dt}, J=6.1,1.6 \mathrm{~Hz}, 2 \mathrm{H}), 7.51(\mathrm{dt}, J=7.7,1.5$ $\mathrm{Hz}, 1 \mathrm{H}), 7.49-7.43(\mathrm{~m}, 1 \mathrm{H}), 3.96(\mathrm{q}, J=6.9 \mathrm{~Hz}, 1 \mathrm{H}), 3.76(\mathrm{t}, J=8.5 \mathrm{~Hz}, 1 \mathrm{H}), 3.69(\mathrm{t}, J=$ $7.3 \mathrm{~Hz}, 1 \mathrm{H}), 3.61-3.51(\mathrm{~m}, 2 \mathrm{H}), 3.49-3.42(\mathrm{~m}, 1 \mathrm{H}), 3.32(\mathrm{~s}, 1 \mathrm{H}), 3.21(\mathrm{dd}, J=11.4,7.5$ $\mathrm{Hz}, 1 \mathrm{H}), 2.63(\mathrm{~s}, 3 \mathrm{H}), 1.50(\mathrm{~d}, J=6.9 \mathrm{~Hz}, 3 \mathrm{H}) .{ }^{13} \mathbf{C} \mathbf{N M R}\left(150 \mathrm{MHz}, \mathrm{CDCl}_{3}\right) \delta 197.44$, 171.27, 141.97, 137.29, 131.34, 128.87, 126.76, 126.57, 66.36, 65.88, 45.58, 42.39, 41.96, 26.26, 20.04. HRMS calcd. for $\mathrm{C}_{15} \mathrm{H}_{19} \mathrm{NO}_{3}{ }^{+}[\mathrm{M}+\mathrm{H}]^{+}:$262.1443, Found: 262.1440 .

tert-butyl (4-(1-morpholino-1-oxopropan-2-yl)phenyl)carbamate (3k): The title compound was BocHN prepared on a $0.200 \mathrm{mmol}$ scale according to General Procedure A. The crude residue was purified by preparative TLC (80\% EtOAc/hexanes). Purification afforded 3k as an off-white solid. (48.9 mg, $73 \%) .{ }^{1} \mathbf{H} \mathbf{~ N M R ~}\left(600 \mathrm{MHz}, \mathrm{CDCl}_{3}\right) \delta$ $7.34(\mathrm{~d}, J=8.1 \mathrm{~Hz}, 2 \mathrm{H}), 7.20-7.15(\mathrm{~m}, 2 \mathrm{H}), 6.47(\mathrm{~s}, 1 \mathrm{H}), 3.80(\mathrm{dq}, J=9.3,6.8$ $\mathrm{Hz}, 2 \mathrm{H}), 3.72-3.66(\mathrm{~m}, 1 \mathrm{H}), 3.58-3.48(\mathrm{~m}, 3 \mathrm{H}), 3.41(\mathrm{dd}, J=12.7,8.1 \mathrm{~Hz}, 1 \mathrm{H})$, $3.32(\mathrm{~d}, J=13.0 \mathrm{~Hz}, 1 \mathrm{H}), 3.20-3.14(\mathrm{~m}, 1 \mathrm{H}), 1.54(\mathrm{~s}, 9 \mathrm{H}), 1.44(\mathrm{~d}, J=6.8 \mathrm{~Hz}, 3 \mathrm{H}) .{ }^{13} \mathrm{C} \mathrm{NMR}(150 \mathrm{MHz}$, 
$\left.\mathrm{CDCl}_{3}\right) \delta 172.28,152.85,137.30,136.24,127.66,119.06,66.75,66.33,60.39,45.97,42.53,28.33,21.04$, 14.18. HRMS calcd. for $\mathrm{C}_{18} \mathrm{H}_{26} \mathrm{~N}_{2} \mathrm{O}_{4}{ }^{+}[\mathrm{M}+\mathrm{H}]^{+}:$335.1971, Found: 335.1978 .

2-(4-methoxyphenyl)-1-morpholinopropan-1-one (31): The title compound was prepared on a 0.200

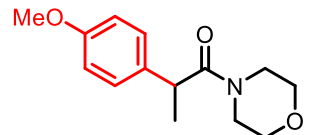
mmol scale according to General Procedure A. The crude residue was purified by preparative TLC (60\% EtOAc/hexanes). Purification afforded 31 as an off-white solid. (27.6 mg, 55\%). Analytical data was in agreement with literature values. ${ }^{15}{ }^{1} \mathbf{H}$ NMR $\left(600 \mathrm{MHz}, \mathrm{CDCl}_{3}\right) \delta 7.23-7.14(\mathrm{~m}, 2 \mathrm{H}), 6.90-6.85(\mathrm{~m}, 2 \mathrm{H}), 3.85-3.78(\mathrm{~m}$, 31 $5 \mathrm{H}), 3.73-3.64(\mathrm{~m}, 1 \mathrm{H}), 3.58-3.49(\mathrm{~m}, 3 \mathrm{H}), 3.45-3.38(\mathrm{~m}, 1 \mathrm{H}), 3.34(\mathrm{dd}, J=$ $10.8,6.2 \mathrm{~Hz}, 1 \mathrm{H}), 3.16(\mathrm{td}, J=8.4,7.6,3.9 \mathrm{~Hz}, 1 \mathrm{H}), 1.44(\mathrm{~d}, J=6.9 \mathrm{~Hz}, 3 \mathrm{H}) .{ }^{13} \mathbf{C ~ N M R}\left(150 \mathrm{MHz}, \mathrm{CDCl}_{3}\right)$ $\delta 172.01,157.97,133.44,127.72,113.89,66.36,65.91,54.80,45.55,41.89,41.85,20.25$. HRMS calcd. for $\mathrm{C}_{14} \mathrm{H}_{19} \mathrm{NO}_{3}{ }^{+}[\mathrm{M}+\mathrm{H}]^{+}: 250.1443$, Found: 250.1443 .

1-morpholino-2-(2-(trifluoromethyl)pyridin-4-yl)propan-1-one (3m): The title compound was prepared

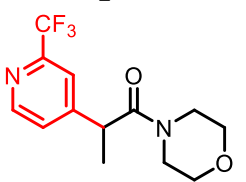
on a $0.200 \mathrm{mmol}$ scale according to General Procedure A. The crude residue was purified by preparative TLC (40\% EtOAc/hexanes). Purification afforded $\mathbf{3 m}$ as an off-white solid. (38.9 mg, 64\%). ${ }^{1} \mathbf{H}$ NMR $\left(600 \mathrm{MHz}, \mathrm{CDCl}_{3}\right) \delta 8.70$ (d, $\left.J=5.0 \mathrm{~Hz}, 1 \mathrm{H}\right), 7.64-7.59$ $(\mathrm{m}, 1 \mathrm{H}), 7.44(\mathrm{dd}, J=5.1,1.7 \mathrm{~Hz}, 1 \mathrm{H}), 4.00(\mathrm{q}, J=6.9 \mathrm{~Hz}, 1 \mathrm{H}), 3.75-3.58(\mathrm{~m}, 5 \mathrm{H}), 3.54$ $3.44(\mathrm{~m}, 1 \mathrm{H}), 3.43-3.30(\mathrm{~m}, 2 \mathrm{H}), 1.53(\mathrm{~d}, J=6.9 \mathrm{~Hz}, 3 \mathrm{H}) .{ }^{13} \mathbf{C ~ N M R}\left(150 \mathrm{MHz}, \mathrm{CDCl}_{3}\right)$ $3 m$ $\delta 169.75,150.93,150.09,148.48(\mathrm{q}, J=34.5 \mathrm{~Hz}), 124.79,120.03,119.00(\mathrm{q}, J=2.7 \mathrm{~Hz})$, 66.30, 65.87, 45.65, 42.09, 41.61, 19.41. ${ }^{19} \mathbf{F}$ NMR (564 MHz, $\mathrm{CDCl}_{3}$ ) $\delta$-68.1845. HRMS calcd. for $\mathrm{C}_{13} \mathrm{H}_{15} \mathrm{~F}_{3} \mathrm{~N}_{2} \mathrm{O}_{2}{ }^{+}[\mathrm{M}+\mathrm{H}]^{+}:$289.1164, Found: 289.1165 .

1-morpholino-2-(pyridin-2-yl)propan-1-one (3n): The title compound was prepared on a $0.200 \mathrm{mmol}$

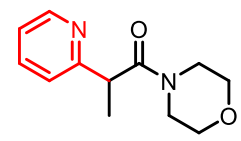
scale according to General Procedure A. The crude residue was purified by preparative TLC (40\% EtOAc/hexanes). Purification afforded $\mathbf{3 n}$ as an off-white solid. (30.8 $\mathrm{mg}$, $70 \%) .{ }^{1} \mathbf{H}$ NMR $\left(600 \mathrm{MHz}, \mathrm{CDCl}_{3}\right) \delta 8.53(\mathrm{ddd}, J=4.9,1.9,0.9 \mathrm{~Hz}, 1 \mathrm{H}), 7.69(\mathrm{td}, J=$ $7.7,1.9 \mathrm{~Hz}, 1 \mathrm{H}), 7.34(\mathrm{dt}, J=7.9,1.1 \mathrm{~Hz}, 1 \mathrm{H}), 7.19(\mathrm{ddd}, J=7.4,4.9,1.1 \mathrm{~Hz}, 1 \mathrm{H}), 4.15$ $3 n$ $(\mathrm{q}, J=7.0 \mathrm{~Hz}, 1 \mathrm{H}), 3.83-3.76(\mathrm{~m}, 1 \mathrm{H}), 3.74-3.65(\mathrm{~m}, 1 \mathrm{H}), 3.60-3.45(\mathrm{~m}, 5 \mathrm{H}), 3.21-3.14$ $(\mathrm{m}, 1 \mathrm{H}), 1.54(\mathrm{~d}, J=6.9 \mathrm{~Hz}, 3 \mathrm{H}) .{ }^{13} \mathbf{C}$ NMR $\left(150 \mathrm{MHz}, \mathrm{CDCl}_{3}\right) \delta 171.50,161.40,149.23,137.17,122.00$, 121.32, 66.79, 66.52, 46.08, 45.79, 42.49, 18.62. HRMS calcd. for $\mathrm{C}_{12} \mathrm{H}_{16} \mathrm{~N}_{2} \mathrm{O}_{2}{ }^{+}[\mathrm{M}+\mathrm{H}]^{+}: 221.1290$, Found: 221.1286.

1-morpholino-2-(pyridin-4-yl)propan-1-one (3o): The title compound was prepared on a $0.200 \mathrm{mmol}$<smiles>CC(C(=O)N1CCOCC1)c1ccncc1</smiles>
scale according to General Procedure A. The crude residue was purified by preparative TLC (40\% EtOAc/hexanes). Purification afforded 3o as an off-white solid (34.8 $\mathrm{mg}$, $80 \%) .{ }^{1} \mathbf{H}$ NMR $\left(600 \mathrm{MHz}, \mathrm{CDCl}_{3}\right) \delta 8.61-8.57(\mathrm{~m}, 2 \mathrm{H}), 7.23-7.19(\mathrm{~m}, 2 \mathrm{H}), 3.87(\mathrm{q}, J=$ $6.9 \mathrm{~Hz}, 1 \mathrm{H}), 3.76(\mathrm{dd}, J=10.5,6.1 \mathrm{~Hz}, 1 \mathrm{H}), 3.70(\mathrm{dd}, J=8.8,6.3 \mathrm{~Hz}, 1 \mathrm{H}), 3.62-3.54(\mathrm{~m}$, 3o $\quad 3 \mathrm{H}), 3.47-3.40(\mathrm{~m}, 1 \mathrm{H}), 3.28(\mathrm{qd}, J=10.7,10.0,2.9 \mathrm{~Hz}, 2 \mathrm{H}), 1.49(\mathrm{~d}, J=6.9 \mathrm{~Hz}, 3 \mathrm{H})$. ${ }^{13} \mathrm{C} \mathrm{NMR}\left(150 \mathrm{MHz}, \mathrm{CDCl}_{3}\right) \delta 170.33,150.03,150.00$ 122.00, 66.30, 65.83, 45.55, $41.98(\mathrm{~d}, J=6.4 \mathrm{~Hz})$, 19.49. HRMS calcd. for $\mathrm{C}_{12} \mathrm{H}_{16} \mathrm{~N}_{2} \mathrm{O}_{2}^{+}[\mathrm{M}+\mathrm{H}]^{+}:$221.1290, Found: 221.1285.

1-morpholino-2-(pyrazin-2-yl)propan-1-one (3p): The title compound was prepared on a $0.200 \mathrm{mmol}$

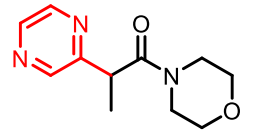
scale according to General Procedure A. The crude residue was purified by preparative TLC (60\% EtOAc/hexanes). Purification afforded $\mathbf{3 p}$ as an off-white solid (26.0 $\mathrm{mg}$, $59 \%) .{ }^{1} \mathbf{H}$ NMR $\left(600 \mathrm{MHz}, \mathrm{CDCl}_{3}\right) \delta 8.67(\mathrm{~s}, 1 \mathrm{H}), 8.50(\mathrm{~s}, 2 \mathrm{H}), 4.21(\mathrm{q}, J=7.0 \mathrm{~Hz}, 1 \mathrm{H})$, $3.81-3.67(\mathrm{~m}, 2 \mathrm{H}), 3.60(\mathrm{t}, J=8.3 \mathrm{~Hz}, 3 \mathrm{H}), 3.53(\mathrm{dq}, J=9.7,4.8,3.8 \mathrm{~Hz}, 2 \mathrm{H}), 3.38-3.31$

3p $\quad(\mathrm{m}, 1 \mathrm{H}), 1.61(\mathrm{~d}, J=7.0 \mathrm{~Hz}, 3 \mathrm{H}) .{ }^{13} \mathbf{C ~ N M R}\left(150 \mathrm{MHz}, \mathrm{CDCl}_{3}\right) \delta 170.15,156.19,143.45$, 143.29, 142.62, 66.34, 66.08, 45.72, 42.92, 42.10, 17.63. HRMS calcd. for $\mathrm{C}_{11} \mathrm{H}_{15} \mathrm{~N}_{3} \mathrm{O}_{2}{ }^{+}[\mathrm{M}+\mathrm{H}]^{+}: 222.1243$, Found: 222.1242. 
2-(7-chloroquinolin-4-yl)-1-morpholinopropan-1-one (3q): The title compound was prepared on an

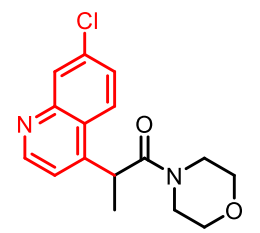
$0.200 \mathrm{mmol}$ scale according to General Procedure A. The crude residue was purified by preparative TLC (60\% EtOAc/hexanes). Purification afforded 3q as an off-white solid $(27.2 \mathrm{mg}, 45 \%) .{ }^{1} \mathbf{H} \mathbf{N M R}\left(600 \mathrm{MHz}, \mathrm{CDCl}_{3}\right) \delta 8.89(\mathrm{~d}, J=4.6 \mathrm{~Hz}, 1 \mathrm{H}), 8.19(\mathrm{~d}, J=2.1$ $\mathrm{Hz}, 1 \mathrm{H}), 8.04(\mathrm{~d}, J=9.0 \mathrm{~Hz}, 1 \mathrm{H}), 7.61(\mathrm{dd}, J=9.0,2.2 \mathrm{~Hz}, 1 \mathrm{H}), 7.31(\mathrm{~d}, J=4.6 \mathrm{~Hz}, 1 \mathrm{H})$, $4.53(\mathrm{q}, J=6.9 \mathrm{~Hz}, 1 \mathrm{H}), 3.84-3.76(\mathrm{~m}, 1 \mathrm{H}), 3.74-3.68(\mathrm{~m}, 1 \mathrm{H}), 3.67-3.58(\mathrm{~m}, 2 \mathrm{H}), 3.44$ (ddd, $J=11.4,6.0,3.0 \mathrm{~Hz}, 1 \mathrm{H}), 3.28$ (ddd, $J=13.2,7.1,2.9 \mathrm{~Hz}, 1 \mathrm{H}), 3.12$ (ddd, $J=11.1$, 3q $\quad 6.9,2.9 \mathrm{~Hz}, 1 \mathrm{H}), 3.05-2.99(\mathrm{~m}, 1 \mathrm{H}), 1.60(\mathrm{~d}, J=6.9 \mathrm{~Hz}, 3 \mathrm{H}) .{ }^{13} \mathbf{C}$ NMR $(150 \mathrm{MHz}$, $\left.\mathrm{CDCl}_{3}\right) \delta 170.60,150.14,148.76,147.11,135.06,129.38,127.85,123.68,123.02,118.77,66.27,65.67$, 45.40, 42.07, 38.40, 18.71. HRMS calcd. for $\mathrm{C}_{16} \mathrm{H}_{17} \mathrm{ClN}_{2} \mathrm{O}_{2}^{+}[\mathrm{M}+\mathrm{H}]^{+}:$305.1057, Found: 305.1056.

3-methyl-7-(1-morpholino-1-oxopropan-2-yl)quinazolin-4(3H)-one $(3 \mathbf{r})$ : The title compound was

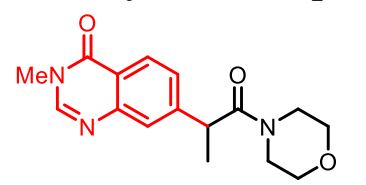
prepared on a $0.200 \mathrm{mmol}$ scale according to General Procedure A. The crude residue was purified by preparative TLC $(10 \% \mathrm{MeOH} / \mathrm{DCM})$. Purification afforded $3 \mathbf{r}$ as an off-white solid (44.7 $\mathrm{mg}, 74 \%)$. ${ }^{1} \mathbf{H}$ NMR $\left(600 \mathrm{MHz}, \mathrm{CDCl}_{3}\right) \delta 8.31(\mathrm{~d}, J$ $=8.3 \mathrm{~Hz}, 1 \mathrm{H}), 8.07(\mathrm{~s}, 1 \mathrm{H}), 7.59(\mathrm{~d}, J=1.7 \mathrm{~Hz}, 1 \mathrm{H}), 7.45(\mathrm{dd}, J=8.3,1.7 \mathrm{~Hz}, 1 \mathrm{H})$, $3 r$ $4.03(\mathrm{q}, J=6.9 \mathrm{~Hz}, 1 \mathrm{H}), 3.79(\mathrm{dt}, J=12.3,7.0 \mathrm{~Hz}, 1 \mathrm{H}), 3.69(\mathrm{q}, J=9.8,8.6 \mathrm{~Hz}$, $1 \mathrm{H}), 3.62(\mathrm{~s}, 3 \mathrm{H}), 3.61-3.48(\mathrm{~m}, 3 \mathrm{H}), 3.49-3.38(\mathrm{~m}, 1 \mathrm{H}), 3.32(\mathrm{~d}, J=13.5 \mathrm{~Hz}$, $1 \mathrm{H}), 3.17(\mathrm{q}, J=8.0 \mathrm{~Hz}, 1 \mathrm{H}), 1.55(\mathrm{~s}, 3 \mathrm{H}) .{ }^{13} \mathrm{C}$ NMR $\left(150 \mathrm{MHz}, \mathrm{CDCl}_{3}\right) \delta 171.18,161.21,148.76,148.50$, 147.29, 127.48, 126.38, 125.87, 120.74, 66.75, 66.29, 46.01, 43.18, 42.44, 34.05, 20.29. HRMS calcd. for $\mathrm{C}_{16} \mathrm{H}_{20} \mathrm{~N}_{3} \mathrm{O}_{3}{ }^{+}[\mathrm{M}+\mathrm{H}]^{+}:$302.1505, Found: 302.1505.

2-methyl-6-(1-morpholino-1-oxopropan-2-yl)isoindolin-1-one (3s): The title compound was prepared

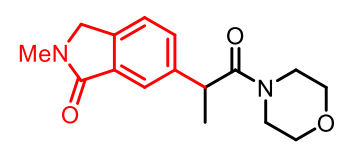
on a $0.200 \mathrm{mmol}$ scale according to General Procedure A. The crude residue was purified by preparative TLC (10\% MeOH/DCM). Purification afforded 3s as an offwhite solid (46.1 mg, 80\%). ${ }^{1} \mathbf{H}$ NMR $\left(600 \mathrm{MHz}, \mathrm{CDCl}_{3}\right) \delta 7.69(\mathrm{~d}, J=1.7 \mathrm{~Hz}, 1 \mathrm{H})$, $7.45(\mathrm{dd}, J=7.9,1.7 \mathrm{~Hz}, 1 \mathrm{H}), 7.41(\mathrm{~d}, J=7.8 \mathrm{~Hz}, 1 \mathrm{H}), 4.36(\mathrm{~s}, 2 \mathrm{H}), 3.96(\mathrm{q}, J=$ $3 \mathbf{s}$ $6.9 \mathrm{~Hz}, 1 \mathrm{H}), 3.76-3.68(\mathrm{~m}, 1 \mathrm{H}), 3.68-3.58(\mathrm{~m}, 2 \mathrm{H}), 3.58-3.45(\mathrm{~m}, 3 \mathrm{H}), 3.42$ (dtd, $J=13.7,6.7,6.3,4.1 \mathrm{~Hz}, 1 \mathrm{H}), 3.28(\mathrm{ddd}, J=13.2,6.1,2.9 \mathrm{~Hz}, 1 \mathrm{H}), 3.19(\mathrm{~s}, 3 \mathrm{H})$, $3.14(\mathrm{ddt}, J=10.8,7.3,3.8 \mathrm{~Hz}, 1 \mathrm{H}), 1.46(\mathrm{~d}, J=6.9 \mathrm{~Hz}, 3 \mathrm{H}) .{ }^{13} \mathbf{C ~ N M R}\left(150 \mathrm{MHz}, \mathrm{CDCl}_{3}\right) \delta 171.78$, 168.31, 142.12, 139.70, 133.60, 129.87, 123.36, 122.56, 66.76, 66.32, 51.85, 45.99, 42.92, 42.38, 29.50, 20.67. HRMS calcd. for $\mathrm{C}_{16} \mathrm{H}_{21} \mathrm{~N}_{2} \mathrm{O}_{3}{ }^{+}[\mathrm{M}+\mathrm{H}]^{+}: 289.1552$, Found: 289.1556.

$N, N$-dibenzyl-2-phenylpropanamide (5a): The title compound was prepared on a $0.200 \mathrm{mmol}$ scale

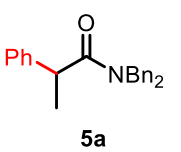
according to General Procedure B. The crude residue was purified by preparative TLC (40\% EtOAc/hexanes). Purification afforded $\mathbf{5 a}$ as an off-white solid (58.0 mg, 88\%). Analytical data was in agreement with literature values. ${ }^{14}{ }^{1} \mathbf{H}$ NMR $\left(600 \mathrm{MHz}, \mathrm{CDCl}_{3}\right) \delta 7.39-7.22(\mathrm{~m}$, $11 \mathrm{H}), 7.17-7.13(\mathrm{~m}, 2 \mathrm{H}), 7.10-7.05(\mathrm{~m}, 2 \mathrm{H}), 5.14(\mathrm{~d}, J=14.8 \mathrm{~Hz}, 1 \mathrm{H}), 4.58(\mathrm{~d}, J=17.1 \mathrm{~Hz}$, $1 \mathrm{H}), 4.16(\mathrm{dd}, J=26.0,16.0 \mathrm{~Hz}, 2 \mathrm{H}), 3.91(\mathrm{q}, J=6.8 \mathrm{~Hz}, 1 \mathrm{H}), 1.52(\mathrm{~d}, J=6.8 \mathrm{~Hz}, 3 \mathrm{H}) .{ }^{13} \mathbf{C} \mathbf{N M R}(150$ $\left.\mathrm{MHz}, \mathrm{CDCl}_{3}\right) \delta 174.36,141.81,137.46,136.70,128.94,128.93,128.75,128.51,128.12,127.56,127.36$, 127.28, 126.97, 126.32, 49.61, 48.41, 43.38, 21.08. HRMS calcd. for $\mathrm{C}_{23} \mathrm{H}_{24} \mathrm{NO}^{+}[\mathrm{M}+\mathrm{H}]^{+}: 330.1858$, Found: 330.1862.

$N, N$-dimethyl-2-phenylpropanamide (5b): The title compound was prepared on a 0.200 mmol scale II according to General Procedure B. The crude residue was purified by preparative TLC (40\% EtOAc/hexanes). Purification afforded $\mathbf{5 b}$ as an off-white solid $(22.7 \mathrm{mg}, 64 \%)$. Analytical data was in agreement with literature values. ${ }^{16}{ }^{1} \mathbf{H} \mathbf{~ N M R}\left(600 \mathrm{MHz}, \mathrm{CDCl}_{3}\right) \delta 7.33(\mathrm{t}, J=7.5$ $\mathrm{Hz}, 2 \mathrm{H}), 7.29(\mathrm{~d}, J=1.6 \mathrm{~Hz}, 1 \mathrm{H}), 7.28(\mathrm{~d}, J=1.3 \mathrm{~Hz}, 1 \mathrm{H}), 7.27-7.20(\mathrm{~m}, 1 \mathrm{H}), 3.90$ (q, $J=$ $6.8 \mathrm{~Hz}, 1 \mathrm{H}), 2.98(\mathrm{~s}, 3 \mathrm{H}), 2.91(\mathrm{~s}, 3 \mathrm{H}), 1.46(\mathrm{~d}, J=6.9 \mathrm{~Hz}, 3 \mathrm{H}) .{ }^{13} \mathrm{C} \mathrm{NMR}\left(150 \mathrm{MHz}, \mathrm{CDCl}_{3}\right) \delta 173.67$, $141.93,128.83,127.33,126.72,43.29,37.15,35.93,20.75$. HRMS calcd. for $\mathrm{C}_{11} \mathrm{H}_{16} \mathrm{NO}^{+}[\mathrm{M}+\mathrm{H}]^{+}$: 178.1232, Found: 178.1234. 
N,2-diphenylpropanamide (5c): The title compound was prepared on a $0.200 \mathrm{mmol}$ scale according to If General Procedure B. The crude residue was purified by preparative TLC (60\% EtOAc/hexanes). Purification afforded $\mathbf{5 c}$ as an off-white solid $(25.0 \mathrm{mg}, 56 \%)$. Analytical data was in agreement with literature values. ${ }^{14} \mathbf{1} \mathbf{H} \mathbf{~ N M R}\left(600 \mathrm{MHz}, \mathrm{CDCl}_{3}\right) \delta 7.48-7.38(\mathrm{~m}$, $6 \mathrm{H}), 7.38-7.27(\mathrm{~m}, 3 \mathrm{H}), 7.12-7.05(\mathrm{~m}, 2 \mathrm{H}), 3.74(\mathrm{q}, J=7.1 \mathrm{~Hz}, 1 \mathrm{H}), 1.63(\mathrm{~d}, J=7.3 \mathrm{~Hz}$, $3 \mathrm{H}) .{ }^{13} \mathrm{C}$ NMR $\left(150 \mathrm{MHz}, \mathrm{CDCl}_{3}\right) \delta 171.78,140.46,137.38,128.73,128.46,127.27,127.15,123.79$, 119.21, 47.71, 18.10. HRMS calcd. For $\mathrm{C}_{15} \mathrm{H}_{15} \mathrm{NO}[\mathrm{M}+\mathrm{H}]^{+}:$:226.1232, Found: 226.1232.

2-phenyl- $N$-(o-tolyl)propenamide (5d): The title compound was prepared on a $0.200 \mathrm{mmol}$ scale

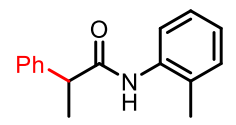

$5 d$ according to General Procedure B. The crude residue was purified by preparative TLC (60\% EtOAc/hexanes). Purification afforded 5d as an off-white solid. (28.5 mg, 60\%) Analytical data was in agreement with literature values. ${ }^{17} \mathbf{H} \mathbf{~ N M R}\left(600 \mathrm{MHz}, \mathrm{CDCl}_{3}\right) \delta$ $7.90(\mathrm{~d}, J=8.1 \mathrm{~Hz}, 1 \mathrm{H}), 7.43(\mathrm{~d}, J=6.3 \mathrm{~Hz}, 4 \mathrm{H}), 7.40-7.30(\mathrm{~m}, 2 \mathrm{H}), 7.20(\mathrm{td}, J=7.8,1.6$ $\mathrm{Hz}, 1 \mathrm{H}), 7.10(\mathrm{~d}, J=7.4 \mathrm{~Hz}, 1 \mathrm{H}), 7.06-7.01(\mathrm{~m}, 1 \mathrm{H}), 6.90(\mathrm{~s}, 1 \mathrm{H}), 3.82(\mathrm{q}, J=7.2 \mathrm{~Hz}, 1 \mathrm{H}), 1.91(\mathrm{~s}, 3 \mathrm{H})$, $1.68(\mathrm{~d}, J=7.3 \mathrm{~Hz}, 3 \mathrm{H}) .{ }^{13} \mathbf{C} \mathbf{N M R}\left(150 \mathrm{MHz}, \mathrm{CDCl}_{3}\right) \delta 171.83,140.53,135.27,129.84,128.80,127.66$, 127.43, 127.30, 126.31, 124.33, 121.63, 47.64, 17.56, 16.66. HRMS calcd. for $\mathrm{C}_{15} \mathrm{H}_{15} \mathrm{NO}^{+}[\mathrm{M}+\mathrm{H}]^{+}$: 240.1388, Found: 240.1387.

$N$-(4-methoxyphenyl)-2-phenylpropanamide (5e): The title compound was prepared on a $0.200 \mathrm{mmol}$

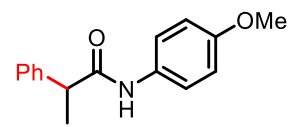

$5 e$ scale according to General Procedure B. The crude residue was purified by preparative TLC (60\% EtOAc/hexanes). Purification afforded 5e as an off-white solid (29.4 mg, $58 \%)$. Analytical data was in agreement with literature values. ${ }^{17} \mathbf{H}$ NMR $(600 \mathrm{MHz}$, $\left.\mathrm{CDCl}_{3}\right) \delta 7.42-7.36(\mathrm{~m}, 4 \mathrm{H}), 7.36-7.30(\mathrm{~m}, 3 \mathrm{H}), 7.06($ broad s, $1 \mathrm{H}), 6.84-6.79(\mathrm{~m}$, 2H), $3.78(\mathrm{~s}, 3 \mathrm{H}), 3.72(\mathrm{q}, J=7.1 \mathrm{~Hz}, 1 \mathrm{H}), 1.62(\mathrm{~d}, J=7.2 \mathrm{~Hz}, 3 \mathrm{H}) .{ }^{13} \mathbf{C} \mathbf{N M R}\left(150 \mathrm{MHz}, \mathrm{CDCl}_{3}\right) \delta 171.74$, $155.93,140.64,130.53,128.66,127.16(\mathrm{~d}, J=31.1 \mathrm{~Hz}), 121.18,113.59,55.01,47.45,18.15$. HRMS calcd. for $\mathrm{C}_{16} \mathrm{H}_{17} \mathrm{NO}_{2}^{+}[\mathrm{M}+\mathrm{H}]^{+}:$256.1338, Found: 256.1336.

$N$-isopropyl-2-phenylpropanamide (5f): The title compound was prepared on an $0.200 \mathrm{mmol}$ scale<smiles>CC(C)NC(=O)C(C)c1ccccc1</smiles>
according to General Procedure B. The crude residue was purified by preparative LCMS. Purification afforded $\mathbf{5 f}$ as an off white solid. (x mg, x\%). ${ }^{1} \mathbf{H}$ NMR ${ }^{1} \mathrm{H}$ NMR $(600 \mathrm{MHz}$, $\left.\mathrm{CDCl}_{3}\right) \delta 7.39-7.33(\mathrm{~m}, 2 \mathrm{H}), 7.33-7.24(\mathrm{~m}, 3 \mathrm{H}), 5.29(\mathrm{~d}, J=22.2 \mathrm{~Hz}, 1 \mathrm{H}), 4.06$ (dhept, $J=$ $8.2,6.5 \mathrm{~Hz}, 1 \mathrm{H}), 3.58(\mathrm{q}, J=7.2 \mathrm{~Hz}, 1 \mathrm{H}), 1.53(\mathrm{~d}, J=7.2 \mathrm{~Hz}, 3 \mathrm{H}), 1.09(\mathrm{~d}, J=6.6 \mathrm{~Hz}, 3 \mathrm{H})$, $1.04(\mathrm{~d}, J=6.6 \mathrm{~Hz}, 3 \mathrm{H}) .{ }^{13} \mathrm{C}$ NMR $\left(150 \mathrm{MHz}, \mathrm{CDCl}_{3}\right) \delta 173.21,141.65,128.87,127.60,127.15,47.21$, 41.39, 22.64, 22.55, 18.66. HRMS calcd. for $\mathrm{C}_{12} \mathrm{H}_{17} \mathrm{NO}^{+}[\mathrm{M}+\mathrm{H}]^{+}:$192.1388, Found: 192.1388.

$N$-cyclobutyl-2-phenylpropanamide (5g): The title compound was prepared on a 0.200 mmol scale II $\square$ according to General Procedure B. The crude residue was purified by preparative TLC (20\% EtOAc/hexanes). Purification afforded $\mathbf{5 g}$ as an off-white solid. (22.8 $\mathrm{mg}, 56 \%) .{ }^{1} \mathbf{H} \mathbf{~ N M R}$ $\left(600 \mathrm{MHz}, \mathrm{CDCl}_{3}\right) \delta 7.39-7.34(\mathrm{~m}, 2 \mathrm{H}), 7.31-7.26(\mathrm{~m}, 2 \mathrm{H}), 5.48(\mathrm{~s}, 1 \mathrm{H}), 4.46-4.25(\mathrm{~m}$, $1 \mathrm{H}), 3.52(\mathrm{q}, J=7.2 \mathrm{~Hz}, 1 \mathrm{H}), 2.36-2.20(\mathrm{~m}, 2 \mathrm{H}), 1.80-1.59(\mathrm{~m}, 4 \mathrm{H}), 1.52(\mathrm{~d}, J=7.2 \mathrm{~Hz}$, 3H). ${ }^{13} \mathrm{C}$ NMR $\left(150 \mathrm{MHz}, \mathrm{CDCl}_{3}\right) \delta 172.70,141.05,128.44,127.18,126.75,46.59,44.37,30.67,30.61$, 18.16, 14.50. HRMS calcd. for $\mathrm{C}_{13} \mathrm{H}_{17} \mathrm{NO}^{+}[\mathrm{M}+\mathrm{H}]^{+}: 204.1388$, Found: 204.1389.

2-phenyl- $N$-(thiophen-2-ylmethyl)propenamide (5h): The title compound was prepared on a $0.200 \mathrm{mmol}$ scale according to General Procedure B. The crude residue was purified by preparative TLC (60\% EtOAc/hexanes). Purification afforded $\mathbf{5 h}$ as an off-white solid (23.3 $\mathrm{mg}$, 48\%). ${ }^{1} \mathbf{H}$ NMR $\left(600 \mathrm{MHz}, \mathrm{CDCl}_{3}\right) \delta$ 7.39-7.33 (m, 2H), 7.33-7.26 (m, 3H), 7.20 (dd, $J$ $=5.1,1.2 \mathrm{~Hz}, 1 \mathrm{H}), 6.92(\mathrm{dd}, J=5.1,3.4 \mathrm{~Hz}, 1 \mathrm{H}), 6.87(\mathrm{dt}, J=3.5,1.1 \mathrm{~Hz}, 1 \mathrm{H}), 5.74(\mathrm{~s}$, $1 \mathrm{H}), 4.64-4.52(\mathrm{~m}, 2 \mathrm{H}), 3.61(\mathrm{q}, J=7.2 \mathrm{~Hz}, 1 \mathrm{H}), 1.57(\mathrm{~s}, 3 \mathrm{H}) .{ }^{13} \mathbf{C} \mathbf{N M R}(150 \mathrm{MHz}$, $\left.\mathrm{CDCl}_{3}\right) \delta^{13} \mathrm{C} \mathrm{NMR}\left(150 \mathrm{MHz}, \mathrm{CDCl}_{3}\right) \delta 173.36,140.65(\mathrm{~d}, J=3.3 \mathrm{~Hz}), 128.51,128.33,127.26,126.90$, 
126.33, 125.15, 124.57, 46.62, 38.04, 18.03 . HRMS calcd. For $\mathrm{C}_{14} \mathrm{H}_{15} \mathrm{NOS}^{+}[\mathrm{M}+\mathrm{H}]^{+}:$246.0953, Found: 246.0952.

$N$-(furan-2-ylmethyl)-2-phenylpropanamide (5i): The title compound was prepared on a $0.200 \mathrm{mmol}$

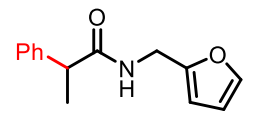

$5 \mathbf{i}$ scale according to General Procedure B. The crude residue was purified by preparative TLC (60\% EtOAc/hexanes). Purification afforded 5i as an off-white solid. (34.5 $\mathrm{mg}$, 75\%). ${ }^{1} \mathbf{H}$ NMR $\left(600 \mathrm{MHz}, \mathrm{CDCl}_{3}\right) \delta 7.39-7.33(\mathrm{~m}, 2 \mathrm{H}), 7.33-7.25(\mathrm{~m}, 4 \mathrm{H}), 6.29(\mathrm{dd}, J$ $=3.2,1.8 \mathrm{~Hz}, 1 \mathrm{H}), 6.12(\mathrm{dd}, J=3.2,0.9 \mathrm{~Hz}, 1 \mathrm{H}), 5.73(\mathrm{~d}, J=6.9 \mathrm{~Hz}, 1 \mathrm{H}), 4.44(\mathrm{dd}, J=$ $15.6,5.7 \mathrm{~Hz}, 1 \mathrm{H}), 4.39-4.33(\mathrm{~m}, 1 \mathrm{H}), 3.60(\mathrm{q}, J=7.2 \mathrm{~Hz}, 1 \mathrm{H}), 1.55(\mathrm{~d}, J=7.2 \mathrm{~Hz}, 3 \mathrm{H})$.

${ }^{13}$ C NMR $\left(150 \mathrm{MHz}, \mathrm{CDCl}_{3}\right) \delta 173.45,150.88,141.61,140.75,128.48,127.21,126.86,109.91,106.67$, 46.59, 36.23, 18.10. HRMS calcd. for $\mathrm{C}_{14} \mathrm{H}_{15} \mathrm{NO}_{2}{ }^{+}[\mathrm{M}+\mathrm{H}]^{+}: 230.1181$, Found: 230.1179 .

$N$-(2-(1H-indol-3-yl)ethyl)-2-phenylpropanamide (5j): The title compound was prepared on a 0.200

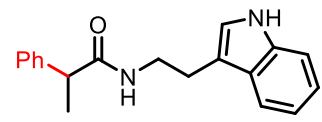

$5 \mathbf{j}$ mmol scale according to General Procedure B. The crude residue was purified by preparative TLC (60\% EtOAc/hexanes). Purification afforded $\mathbf{5 j}$ as an off-white solid. (30.2 mg, 52\%). ${ }^{1} \mathbf{H}$ NMR $\left(600 \mathrm{MHz}, \mathrm{CDCl}_{3}\right) \delta 7.95(\mathrm{~s}, 1 \mathrm{H}), 7.55$ (dq, $J=7.8$, $0.9 \mathrm{~Hz}, 1 \mathrm{H}), 7.37(\mathrm{dt}, J=8.3,0.9 \mathrm{~Hz}, 1 \mathrm{H}), 7.34-7.25(\mathrm{~m}, 3 \mathrm{H}), 7.22(\mathrm{ddt}, J=9.2$, $6.9,1.5 \mathrm{~Hz}, 3 \mathrm{H}), 7.12(\mathrm{ddd}, J=8.0,7.0,1.0 \mathrm{~Hz}, 1 \mathrm{H}), 6.72(\mathrm{~d}, J=2.3 \mathrm{~Hz}, 1 \mathrm{H}), 5.41$ (s, $1 \mathrm{H}), 3.58(\mathrm{dq}, J=12.9,6.4 \mathrm{~Hz}, 1 \mathrm{H}), 3.54-3.46(\mathrm{~m}, 2 \mathrm{H}), 2.95-2.84(\mathrm{~m}, 2 \mathrm{H}), 1.52(\mathrm{~d}, J=7.2 \mathrm{~Hz}, 3 \mathrm{H})$. ${ }^{13} \mathrm{C}$ NMR $\left(150 \mathrm{MHz}, \mathrm{CDCl}_{3}\right.$ 174.04, 141.52, 136.31, 128.84, 127.69, 127.22, 127.13, 122.17, 121.99, $119.49,118.71,112.88,111.14,47.21,39.70,25.10,18.40$. HRMS calcd. for $\mathrm{C}_{19} \mathrm{H}_{20} \mathrm{~N}_{2} \mathrm{O}^{+}[\mathrm{M}+\mathrm{H}]^{+}$: 293.1654, Found: 293.1654.

ethyl 2-(4-bromophenyl)phenylpropanoate (5k): The title compound was prepared on a $0.200 \mathrm{mmol}$ scale according to General Procedure B. The crude residue was purified by preparative TLC (10\% EtOAc/hexanes). Purification afforded 5k as an off-white solid. (32.7 $\mathrm{mg}$, 64\%). ${ }^{1} \mathbf{H}$ NMR $\left(600 \mathrm{MHz}, \mathrm{CDCl}_{3}\right) \delta 7.49-7.44(\mathrm{~m}, 2 \mathrm{H}), 7.23-7.18(\mathrm{~m}, 2 \mathrm{H}), 4.20-4.08$ $(\mathrm{m}, 2 \mathrm{H}), 3.68(\mathrm{q}, J=7.2 \mathrm{~Hz}, 1 \mathrm{H}), 1.50(\mathrm{~d}, J=7.2 \mathrm{~Hz}, 3 \mathrm{H}), 1.23(\mathrm{t}, J=7.1 \mathrm{~Hz}, 3 \mathrm{H}) .{ }^{13} \mathbf{C}$ NMR $\left(150 \mathrm{MHz}, \mathrm{CDCl}_{3}\right) \delta 174.07,139.64,131.68,129.27,121.01,60.92,45.04,18.47$, 14.10. HRMS calcd. for $\mathrm{C}_{12} \mathrm{H}_{13} \mathrm{BrO}_{2}{ }^{+}[\mathrm{M}+\mathrm{H}]^{+}:$257.0177, Found: 257.0182.

tert-butyl 2-(4-bromophenyl)phenylpropanoate (5l): The title compound was prepared on a $0.200 \mathrm{mmol}$ scale according to General Procedure B. The crude residue was purified by preparative TLC (10\% EtOAc/hexanes). Purification afforded $\mathbf{5 l}$ as an off-white solid in a 16:1 mixture of isomers. (33.6 mg, 59\%). ${ }^{1} \mathbf{H}$ NMR $\left(600 \mathrm{MHz}, \mathrm{CDCl}_{3}\right) \delta 7.48-7.43(\mathrm{~m}, 2 \mathrm{H})$, $7.22-7.16(\mathrm{~m}, 2 \mathrm{H}), 3.59(\mathrm{q}, J=7.1 \mathrm{~Hz}, 1 \mathrm{H}), 1.44(\mathrm{~d}, J=3.9 \mathrm{~Hz}, 3 \mathrm{H}), 1.41(\mathrm{~s}, 9 \mathrm{H}) .{ }^{13} \mathbf{C}$ NMR $\left(150 \mathrm{MHz}, \mathrm{CDCl}_{3}\right) \delta 173.34,140.15,131.56,129.21,120.76,80.78,45.97,27.91$, 18.39. HRMS calcd. for $\mathrm{C}_{9} \mathrm{H}_{8} \mathrm{BrO}_{2}{ }^{+}[\mathrm{M}-\mathrm{tBu}]^{+}:$228.9864, Found: 228.9861 .

1-morpholino-2-phenylbutan-1-one (5m): The title compound was prepared on a $0.100 \mathrm{mmol}$ scale

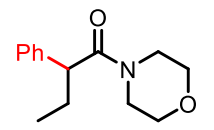
according to General Procedure C. The crude residue was purified by preparative LCMS. Purification afforded $\mathbf{5 m}$ as an off-white solid. (11 $\mathrm{mg}, 47 \%)$. Analytical data was in agreement with literature values. ${ }^{18}{ }^{1} \mathbf{H}$ NMR $\left(600 \mathrm{MHz}, \mathrm{CDCl}_{3}\right) \delta$ 7.37-7.29 (m, 2H), 7.26 $(\mathrm{dtd}, J=7.2,2.1,1.1 \mathrm{~Hz}, 3 \mathrm{H}), 3.80(\mathrm{dd}, J=11.1,5.7 \mathrm{~Hz}, 1 \mathrm{H}), 3.67(\mathrm{ddd}, J=12.1,9.1,4.4$ $5 m$ $\mathrm{Hz}, 1 \mathrm{H}), 3.57$ (t, $J=7.2 \mathrm{~Hz}, 1 \mathrm{H}), 3.54-3.48(\mathrm{~m}, 3 \mathrm{H}), 3.48-3.43(\mathrm{~m}, 1 \mathrm{H}), 3.41-3.34(\mathrm{~m}, 1 \mathrm{H})$, 3.12 (ddd, $J=10.9,7.3,2.9 \mathrm{~Hz}, 1 \mathrm{H}), 2.14$ (dt, $J=13.7,7.3 \mathrm{~Hz}, 1 \mathrm{H}), 1.78$ (dt, $J=13.6,7.2 \mathrm{~Hz}, 1 \mathrm{H}$ ), 0.90 $(\mathrm{t}, J=7.4 \mathrm{~Hz}, 3 \mathrm{H}) .{ }^{13} \mathrm{C}$ NMR $\left(150 \mathrm{MHz}, \mathrm{CDCl}_{3}\right) \delta 171.69,140.10,128.84,127.77,126.96,66.83,66.38$, 50.63, 46.05, 42.39, 27.90, 12.39. HRMS calcd. for $\mathrm{C}_{14} \mathrm{H}_{19} \mathrm{NO}_{2}{ }^{+}[\mathrm{M}+\mathrm{H}]^{+}:$234.1494, Found: 234.1498. 
1-morpholino-2-phenylhexan-1-one (5n): The title compound was prepared on a $0.100 \mathrm{mmol}$ scale

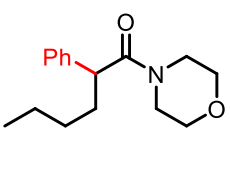
according to General Procedure C. The crude residue was purified by preparative LCMS. Purification afforded $\mathbf{5 n}$ as an off-white solid. (11 mg, 42\%). Analytical data was in agreement with literature values. ${ }^{18}{ }^{1} \mathbf{H}$ NMR $\left(600 \mathrm{MHz}, \mathrm{CDCl}_{3}\right) \delta 7.35-7.30(\mathrm{~m}, 2 \mathrm{H})$, 7.27-7.24 (m, 3H), $3.79(\mathrm{dd}, J=11.2,5.7 \mathrm{~Hz}, 1 \mathrm{H}), 3.71-3.61(\mathrm{~m}, 2 \mathrm{H}), 3.58-3.43(\mathrm{~m}, 4 \mathrm{H})$, 3.38 (ddd, $J=13.5,5.9,3.0 \mathrm{~Hz}, 1 \mathrm{H}), 3.12$ (ddd, $J=10.9,7.3,2.9 \mathrm{~Hz}, 1 \mathrm{H}), 2.12$ (dddd, $J$ $=13.5,10.6,7.5,4.6 \mathrm{~Hz}, 1 \mathrm{H}), 1.83-1.62(\mathrm{~m}, 2 \mathrm{H}), 1.43-1.24(\mathrm{~m}, 3 \mathrm{H}), 1.24-1.10(\mathrm{~m}, 1 \mathrm{H}), 0.93-0.84(\mathrm{~m}$, 3H). ${ }^{13} \mathrm{C}$ NMR $\left(150 \mathrm{MHz}, \mathrm{CDCl}_{3}\right) \delta 171.78,140.34,128.84,127.71,126.91,66.82,66.38,48.83,46.06$, 42.41, 34.59, 30.01, 22.69, 13.98. HRMS calcd. for $\mathrm{C}_{16} \mathrm{H}_{23} \mathrm{NO}_{2}{ }^{+}[\mathrm{M}+\mathrm{H}]^{+}:$262.1807, Found: 262.1813.

4-methyl-1-morpholino-2-phenylpentan-1-one (5o): The title compound was prepared on a $0.100 \mathrm{mmol}$<smiles>CC(C)CC(C(=O)N1CCOCC1)c1ccccc1</smiles>
scale according to General Procedure $\mathrm{C}$ from the corresponding $Z$-alkene starting material. The crude residue was purified by preparative LCMS. Purification afforded 50 as an offwhite solid. (4.2 mg, 16\%). Analytical data was in agreement with literature values. ${ }^{18}{ }^{1} \mathbf{H}$ NMR $\left(600 \mathrm{MHz}, \mathrm{CDCl}_{3}\right) \delta 7.33(\mathrm{td}, J=7.4,1.4 \mathrm{~Hz}, 2 \mathrm{H}), 7.28-7.22(\mathrm{~m}, 3 \mathrm{H}), 3.79(\mathrm{q}, J=$ 8.4, 7.8 Hz, 2H), $3.68(\mathrm{~d}, J=11.0 \mathrm{~Hz}, 1 \mathrm{H}), 3.52(\mathrm{ddd}, J=16.3,11.3,7.0 \mathrm{~Hz}, 4 \mathrm{H}), 3.42(\mathrm{~d}$, $J=13.4 \mathrm{~Hz}, 1 \mathrm{H}), 3.15(\mathrm{dd}, J=13.3,5.7 \mathrm{~Hz}, 1 \mathrm{H}), 1.62(\mathrm{dt}, J=13.7,6.9 \mathrm{~Hz}, 1 \mathrm{H}), 1.51(\mathrm{dp}, J=13.5,6.7$ $\mathrm{Hz}, 2 \mathrm{H}), 0.93(\mathrm{dd}, J=26.0,6.6 \mathrm{~Hz}, 6 \mathrm{H}) .{ }^{13} \mathbf{C} \mathbf{N M R}\left(150 \mathrm{MHz}, \mathrm{CDCl}_{3}\right) \delta 171.75,140.38,128.87,127.71$, 126.90, 66.83, 66.40, 46.23, 46.07, 43.88, 42.43, 25.62, 22.74, 22.56. HRMS calcd. for $\mathrm{C}_{16} \mathrm{H}_{23} \mathrm{NO}_{2}{ }^{+}$ $[\mathrm{M}+\mathrm{H}]^{+}:$262.1807, Found: 262.1815 .

4-methyl-1-morpholino-2-phenylpentan-1-one (5p): The title compound was prepared on a $0.100 \mathrm{mmol}$

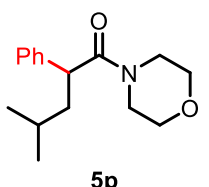
scale according to General Procedure $\mathrm{C}$ from the corresponding $E$-alkene starting material. The crude residue was purified by preparative LCMS. Purification afforded $\mathbf{5 p}$ as an offwhite solid. (4.8 mg, 18\%). Analytical data was in agreement with literature values. ${ }^{18}{ }^{1} \mathbf{H}$ NMR $\left(600 \mathrm{MHz}, \mathrm{CDCl}_{3}\right) \delta 7.33(\mathrm{td}, J=7.4,1.4 \mathrm{~Hz}, 2 \mathrm{H}), 7.28-7.22(\mathrm{~m}, 3 \mathrm{H}), 3.79(\mathrm{q}, J=$ 8.4, 7.8 Hz, 2H), $3.68(\mathrm{~d}, J=11.0 \mathrm{~Hz}, 1 \mathrm{H}), 3.52(\mathrm{ddd}, J=16.3,11.3,7.0 \mathrm{~Hz}, 4 \mathrm{H}), 3.42(\mathrm{~d}$, $J=13.4 \mathrm{~Hz}, 1 \mathrm{H}), 3.15(\mathrm{dd}, J=13.3,5.7 \mathrm{~Hz}, 1 \mathrm{H}), 1.62(\mathrm{dt}, J=13.7,6.9 \mathrm{~Hz}, 1 \mathrm{H}), 1.51(\mathrm{dp}, J=13.5,6.7$ $\mathrm{Hz}, 2 \mathrm{H}), 0.93(\mathrm{dd}, J=26.0,6.6 \mathrm{~Hz}, 6 \mathrm{H}) .{ }^{13} \mathbf{C} \mathbf{~ N M R}\left(150 \mathrm{MHz}, \mathrm{CDCl}_{3}\right) \delta 171.75,140.38,128.87,127.71$, 126.90, 66.83, 66.40, 46.23, 46.07, 43.88, 42.43, 25.62, 22.74, 22.56. HRMS calcd. for $\mathrm{C}_{16} \mathrm{H}_{23} \mathrm{NO}_{2}{ }^{+}$ $[\mathrm{M}+\mathrm{H}]^{+}:$262.1807, Found: 262.1814.

1-morpholino-2,3-diphenylpropan-1-one (5q): The title compound was prepared on a $0.100 \mathrm{mmol}$ scale

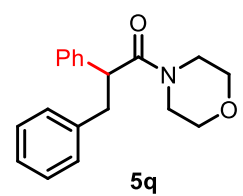
according to General Procedure C. The crude residue was purified by preparative LCMS. Purification afforded $\mathbf{5 q}$ as an off-white solid. (7.0 $\mathrm{mg}, 24 \%)$. Analytical data was in agreement with literature values. ${ }^{18}{ }^{1} \mathbf{H}$ NMR $\left(600 \mathrm{MHz}, \mathrm{CDCl}_{3}\right) \delta 7.35-7.29(\mathrm{~m}, 3 \mathrm{H})$, $7.28-7.15(\mathrm{~m}, 5 \mathrm{H}), 7.14-7.05(\mathrm{~m}, 2 \mathrm{H}), 3.97(\mathrm{td}, J=7.1,3.1 \mathrm{~Hz}, 1 \mathrm{H}), 3.70(\mathrm{dt}, J=11.4$, $5.2 \mathrm{~Hz}, 1 \mathrm{H}), 3.54(\mathrm{tq}, J=16.6,8.0 \mathrm{~Hz}, 4 \mathrm{H}), 3.40-3.22(\mathrm{~m}, 3 \mathrm{H}), 3.14-3.05(\mathrm{~m}, 1 \mathrm{H}), 2.99$ $(\mathrm{dt}, J=13.2,5.0 \mathrm{~Hz}, 1 \mathrm{H}) .{ }^{13} \mathbf{C} \mathbf{N M R}\left(150 \mathrm{MHz}, \mathrm{CDCl}_{3}\right) \delta 171.15,139.81,139.51,129.22,128.81,128.25$, 127.88, 127.16, 126.24, 66.74, 66.24, 50.84, 46.13, 42.48, 40.98. HRMS calcd. for $\mathrm{C}_{19} \mathrm{H}_{21} \mathrm{NO}_{2}{ }^{+}[\mathrm{M}+\mathrm{H}]^{+}$: 296.1651, Found: 296.1653. 


\section{Unsuccessful Coupling Partners}

The following substrates gave less than $15 \%$ yield and/or poor selectivity for $\alpha$-hydroarylation. (With methyl vinyl ketone, reduction to methyl ethyl ketone was observed as the major pathway).

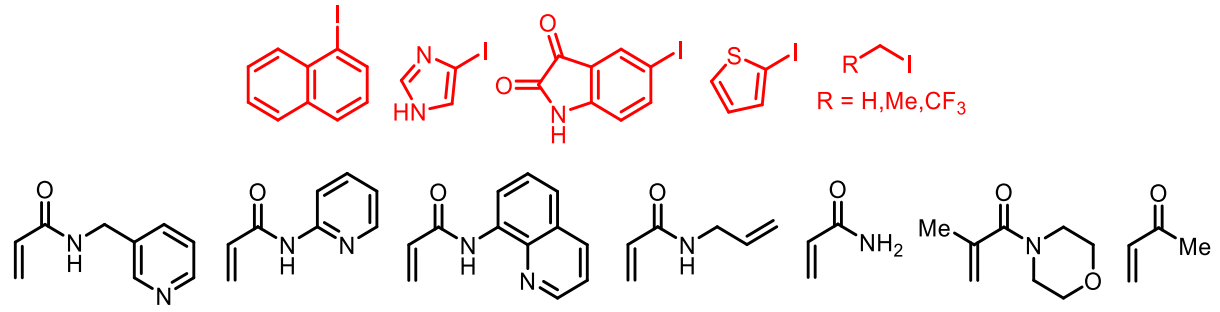

Figure S3. Unsuccessful coupling partners.

\section{0-mmol-Scale Reaction}

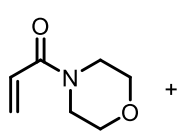

$1 \mathrm{a}$

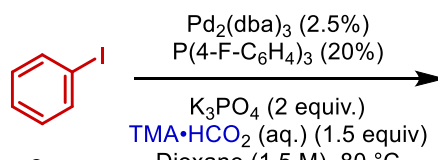

$2 a$

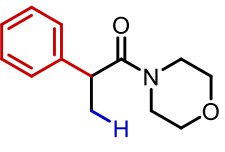

$3 \mathbf{a}$

$\alpha: \beta$

Scheme S5. 10-mmol-scale reaction

To a 50-mL flask containing a stir bar were added $\mathrm{K}_{3} \mathrm{PO}_{4}(85 \mathrm{mg}, 0.40 \mathrm{mmol}), \mathrm{Pd}_{2}(\mathrm{dba})_{2}(230 \mathrm{mg}, 0.25$ $\mathrm{mmol})$, tris(4-fluorophenyl)phosphane $(630 \mathrm{mg}, 2.0 \mathrm{mmol})$, and 1,4-dioxane $(6.6 \mathrm{~mL})$. This solution was allowed to stir briefly ( $<3 \mathrm{~min})$ at room temperature until the solid components dissolved. To this solution were added iodobenzene $(2.4 \mathrm{~g}, 1.3 \mathrm{~mL}, 12 \mathrm{mmol})$, 4-acryloylmorpholine (1.4 g, $1.3 \mathrm{~mL}, 10.0 \mathrm{mmol})$, and tetramethylammonium formate $(30 \mathrm{wt} \%, 5.3 \mathrm{~mL}, 15.0 \mathrm{mmol})$. The flask was sealed with a septum and placed in an oil bath that was pre-heated to $80{ }^{\circ} \mathrm{C}$ for $2 \mathrm{~h}$. After $2 \mathrm{~h}$, the flask was cooled to room temperature. A small aliquot was removed and dissolved in $\mathrm{CDCl}_{3}$ for ${ }^{1} \mathrm{H} \mathrm{NMR}$ analysis $(600 \mathrm{MHz})$, which showed a crude product ratio of $\alpha: \beta=23: 1$. The solution was added to a separatory funnel and water ( 5 $\mathrm{mL})$ was added. The reaction mixture was extracted with EtOAc $(3 \times 10 \mathrm{~mL})$. The combined organic extracts were washed once with brine $(10 \mathrm{~mL})$, dried over $\mathrm{Mg}_{2} \mathrm{SO}_{4}$, and concentrated under vacuum to give a bright yellow oil. This was purified by column chromatography ( $40 \% \mathrm{EtOAc/} \mathrm{hexanes)} \mathrm{to} \mathrm{give} \mathbf{3 a}$ as an orange oil (1.59 g, 73\%). 


\section{Racemization Experiment}
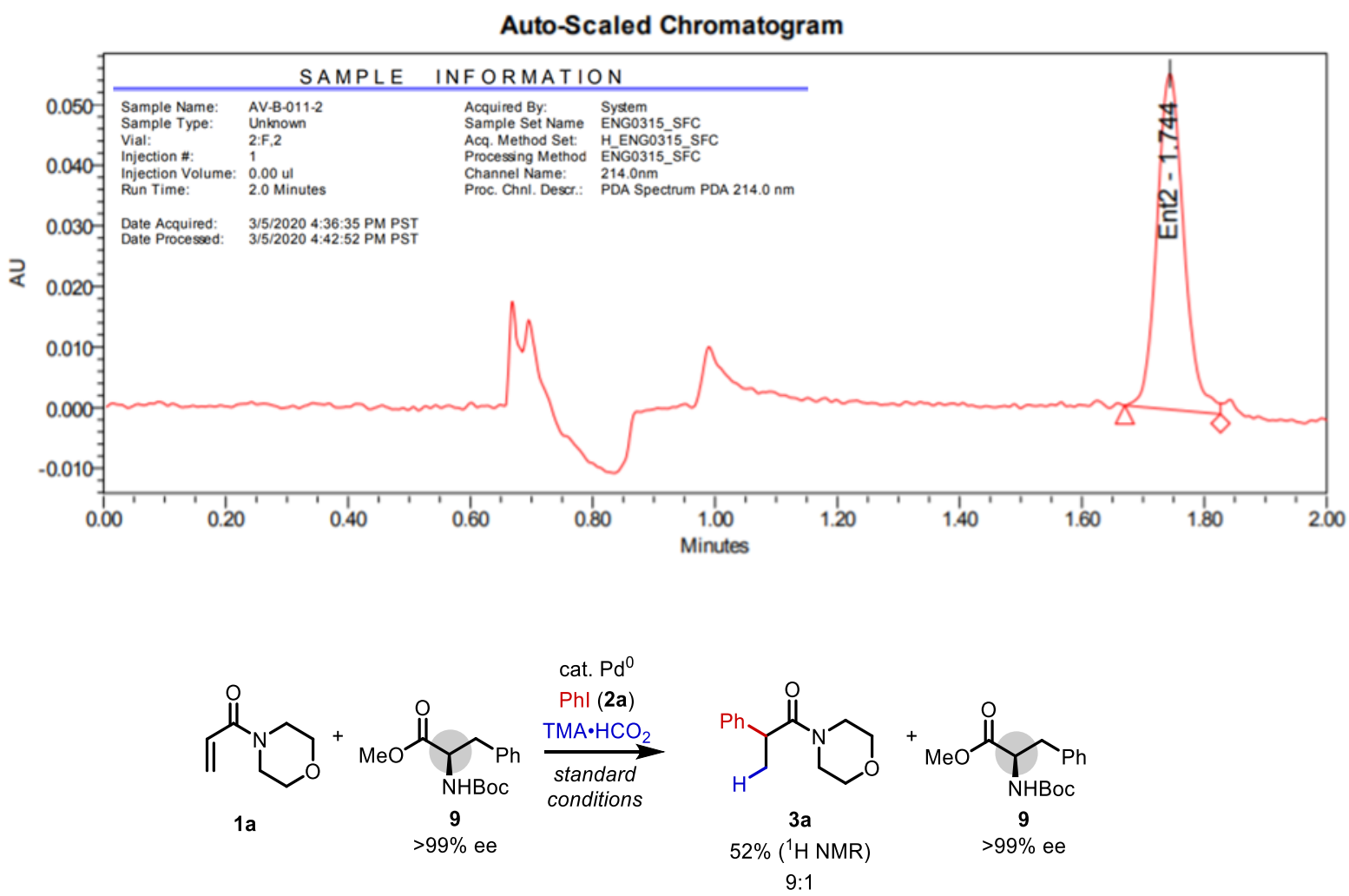

Scheme S6. Racemization test with additive 9

General Procedure: To a two-dram vial containing a stir bar was added $\mathrm{K}_{3} \mathrm{PO}_{4}$ ( $85 \mathrm{mg}, 0.40 \mathrm{mmol}$ ). Using a micropipette, aryl iodide $(0.240 \mathrm{mmol})$, 4-acryloylmorpholine $(28.2 \mathrm{mg}, 25.2 \mu \mathrm{L}, 0.200 \mathrm{mmol})$, tetramethylammonium formate $(30 \mathrm{wt} \%, 100 \mu \mathrm{L}, 0.290 \mathrm{mmol})$, and premixed solution of palladium (4.58 $\mathrm{mg}, 0.005 \mathrm{mmol}$ ) and tris(4-fluoro-phenyl)phosphane $(12.7 \mathrm{mg}, 0.040 \mathrm{mmol})$ in 1,4-dioxane $(0.133 \mathrm{~mL})$ were added sequentially to the vial. To this vial was added $9(27.9 \mathrm{mg}, 0.100 \mathrm{mmol},>99 \%$ ee $)$. The vial was sealed and placed in a pre-heated heating block at $60^{\circ} \mathrm{C}$ for $1 \mathrm{~h}$. After $1 \mathrm{~h}$, the vial was cooled to room temperature. A small aliquot was removed and dissolved in $\mathrm{CDCl}_{3}$ for ${ }^{1} \mathrm{H} \mathrm{NMR}$ analysis $(600 \mathrm{MHz})$. The NMR sample was recombined with the reaction mixture, and the solution was diluted with MeCN to approximately $1 \mathrm{mg} / \mathrm{mL}$ and run on $2 \mathrm{D}$ chiral-SFC to determine ee. Retention time $1.61 \mathrm{~min}$ (minor) and 1.74 min (major). 
A

Auto-Scaled Chromatogram



B

Auto-Scaled Chromatogram

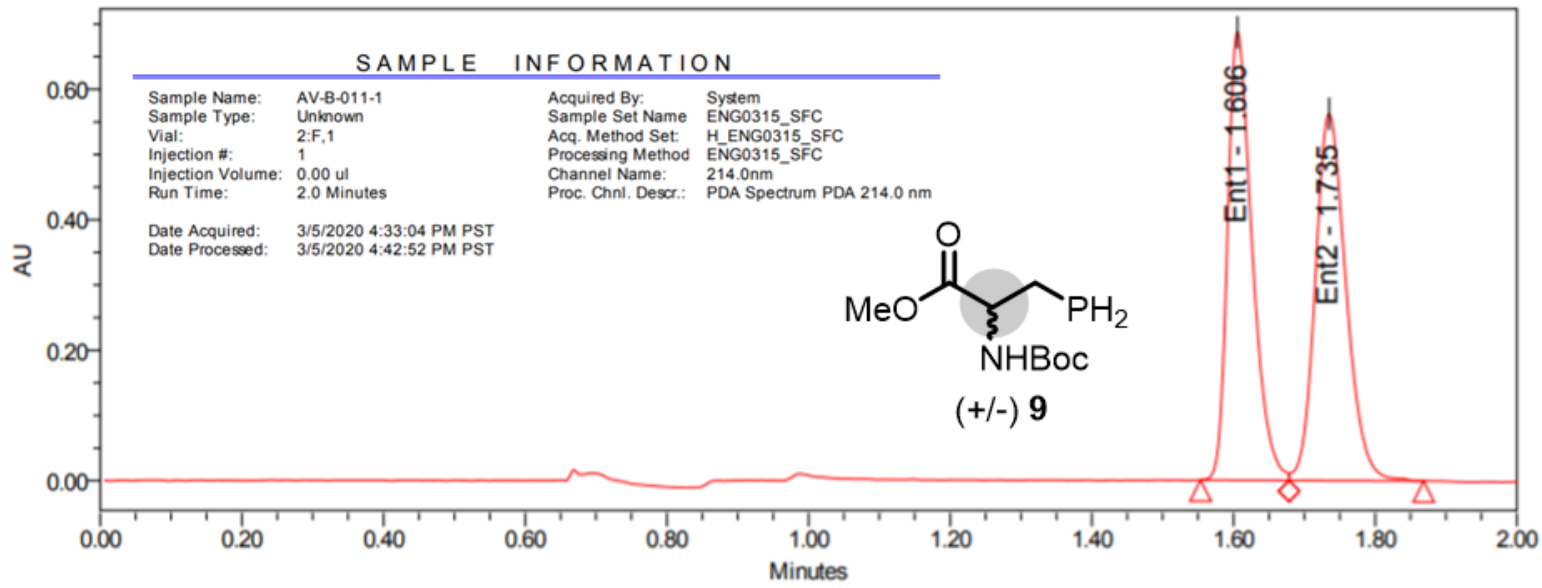

C Auto-Scaled Chromatogram

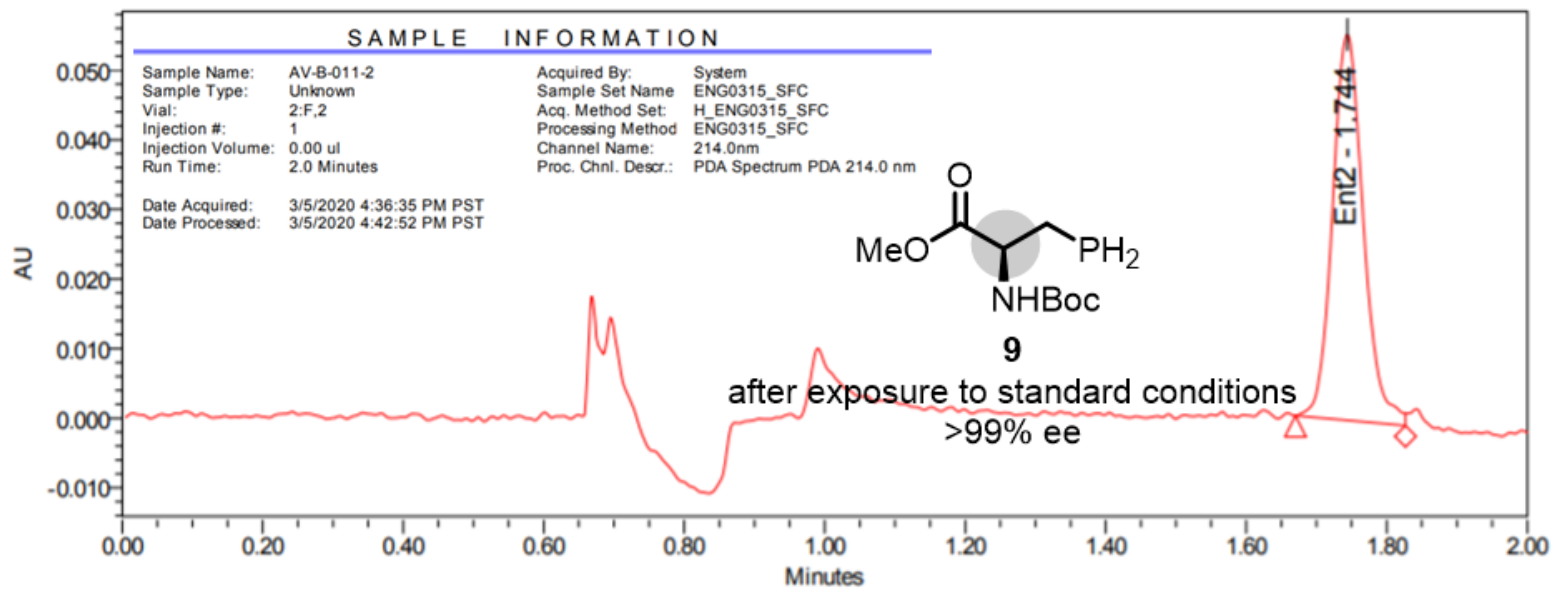

Figure S4. SFC traces for racemic 9 (A), enantiopure 9 (B), and enantioenriched recovered 9 after reaction conditions $(\mathrm{C})$. 


\section{Base-Sensitive Procedure and NMR Spectra}

General Procedure for Base-Sensitive Substrates

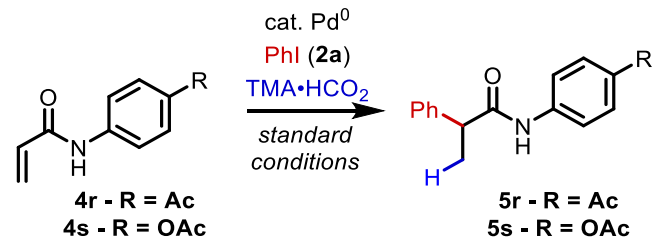

Scheme S7. General procedure for base-sensitive substrates $4 \mathbf{s}$ and $\mathbf{4 t}$

To a one-dram vial containing a stir bar was added $\mathrm{K}_{3} \mathrm{PO}_{4}(43 \mathrm{mg}, 0.20 \mathrm{mmol})$ and 1,3,5-trimethoxybenzene (11.2 mg, $0.067 \mathrm{mmol})$. Using a micropipette, aryl iodide $(0.240 \mathrm{mmol})$, alkene $(0.200 \mathrm{mmol})$, tetramethylammonium formate $(30 \mathrm{wt} \%, 100 \mu \mathrm{L}, 0.290 \mathrm{mmol})$, and Pd/Ligand stock solution $(133 \mu \mathrm{L})$ were added sequentially to the vial. The vial was sealed and placed in a pre-heated heating block at $80{ }^{\circ} \mathrm{C}$ for $1 \mathrm{~h}$. After $1 \mathrm{~h}$, the vial was cooled to room temperature and the contents were dissolved in $\mathrm{CDCl}_{3}$ for ${ }^{1} \mathrm{H}$ NMR analysis (600 MHz). Yields are referenced to 1,3,5-trimethoxybenzene as internal standard.

\section{NMR Spectra of Base Sensitive Substrates}
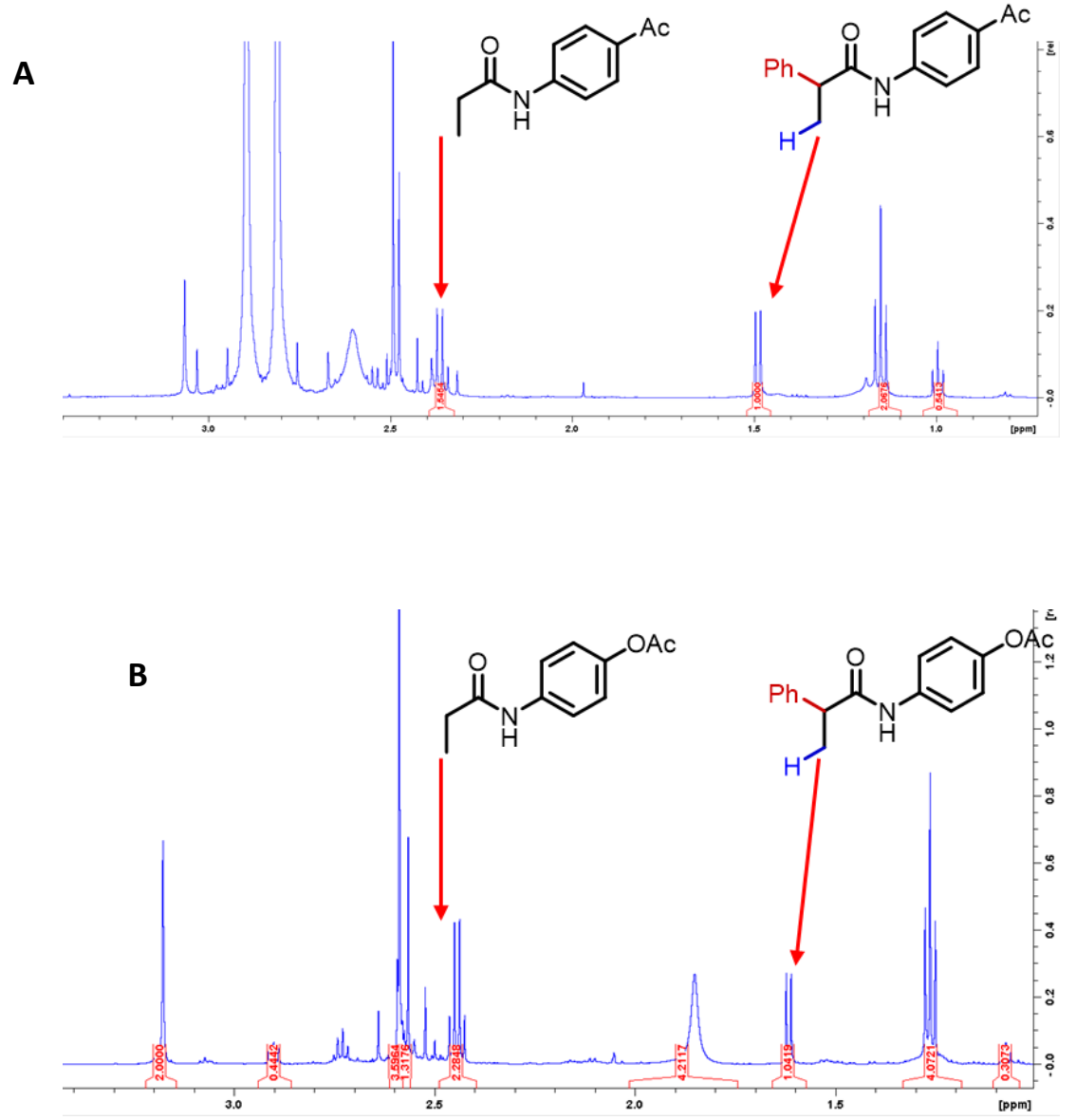

Figure S5. Crude NMR spectra of (A) $5 r$ and (B) $5 s$ 


\section{MECHANISTIC EXPERIMENTS}

\section{Synthesis of Compounds 6-8}

$6^{8}$ and $7^{9}$ were prepared according to literature procedures.

Synthesis and Characterization of Complex $\mathbf{8}$

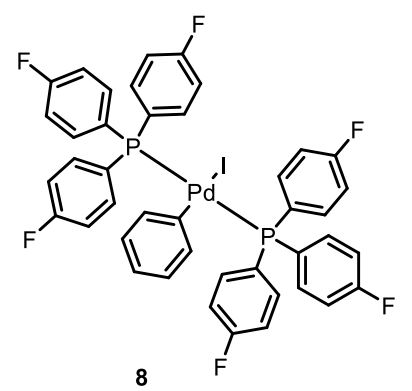

To a $20-\mathrm{mL}$ screw cap vial was added $\mathrm{Pd}_{2}(\mathrm{dba})_{3}(458 \mathrm{mg}, 0.500 \mathrm{mmol})$, tris(4-fluorophenyl)phosphane (648 mg, $2.05 \mathrm{mmol}$ ), and DCM (12 mL). Once homogeneous, this solution was layered with a solution of iodobenzene $(613 \mu \mathrm{L}, 5.50 \mathrm{mmol})$ in $\mathrm{Et}_{2} \mathrm{O}$ and kept in a $-10{ }^{\circ} \mathrm{C}$ freezer overnight. The precipitate was filtered and dried under vacuum to give $187 \mathrm{mg}(0.20 \mathrm{mmol}$, $40 \%$ yield) of monomeric oxidative addition complex as yellow crystals of $\mathrm{x}$ ray diffraction quality. ${ }^{1} \mathbf{H} \mathbf{~ N M R}\left(600 \mathrm{MHz}, \mathrm{CDCl}_{3}\right) \delta 7.44$ (ddt, $J=10.4,5.3$, $3.7 \mathrm{~Hz}, 11 \mathrm{H}), 7.02-6.95(\mathrm{~m}, 11 \mathrm{H}), 6.56(\mathrm{dtd}, J=7.8,2.3,1.1 \mathrm{~Hz}, 2 \mathrm{H}), 6.53-$ $6.46(\mathrm{~m}, 1 \mathrm{H}), 6.36(\mathrm{t}, J=7.4 \mathrm{~Hz}, 2 \mathrm{H}) .{ }^{13} \mathbf{C ~ N M R}\left(150 \mathrm{MHz}, \mathrm{CDCl}_{3}\right) \delta 163.82$ $(\mathrm{d}, J=252.4 \mathrm{~Hz}), 159.34,136.70(\mathrm{q}, J=7.5 \mathrm{~Hz}), 135.76(\mathrm{t}, J=5.0 \mathrm{~Hz}), 128.32,127.26(\mathrm{td}, J=24.5,3.4$ $\mathrm{Hz}), 122.53,115.41(\mathrm{dt}, J=21.3,5.8 \mathrm{~Hz}) .{ }^{19} \mathbf{F} \mathbf{N M R}\left(376 \mathrm{MHz}, \mathrm{CDCl}_{3}\right) \delta-109.52$. HRMS calcd. for $\mathrm{C}_{42} \mathrm{H}_{29} \mathrm{~F}_{6} \mathrm{P}_{2} \mathrm{Pd}^{+}[\mathrm{M}-\mathrm{I}]^{+}: 811.0705$, Found: 811.0712. X-ray (single-crystal) If insufficiently pure, crystals of X-ray quality can be grown by recrystallization from hexanes (CCDC 1987658). ${ }^{13}$

\section{General Procedure for Stoichiometric Study}
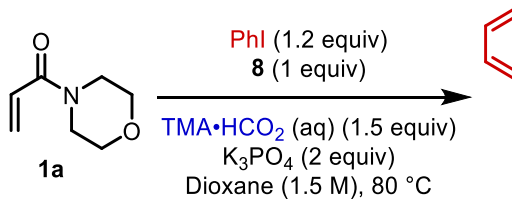

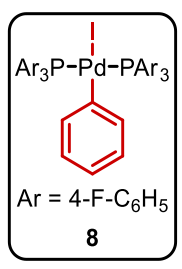

Scheme S8. Stoiciometric study.

To a one-dram vial containing a stir bar was added $\mathrm{K}_{3} \mathrm{PO}_{4}(43 \mathrm{mg}, 0.20 \mathrm{mmol})$ and 1,3,5-trimethoxybenzene $(5.6 \mathrm{mg}, 0.033 \mathrm{mmol})$. Using a micropipette, aryl iodide $(0.140 \mathrm{mmol})$, 4-acryloylmorpholine $(14.1 \mathrm{mg}$, $12.6 \mu \mathrm{L}, 0.100 \mathrm{mmol})$, tetramethylammoniumformate $(30 \mathrm{wt} \%, 53 \mu \mathrm{L}, 0.150 \mathrm{mmol})$, and $8(106 \mathrm{mg}, 0.110$ $\mathrm{mmol}$ in $66 \mathrm{uL}$ Dioxane) was added sequentially to the vial. The vial was sealed and placed in a pre-heated heating block at $80{ }^{\circ} \mathrm{C}$ for 60 minutes. After 60 minutes, the vial was cooled to room temperature and the contents were dissolved in $\mathrm{CDCl}_{3}$ for ${ }^{1} \mathrm{H}$ NMR analysis $(600 \mathrm{MHz})$. Yields are referenced to 1,3,5trimethoxybenzene as internal standard.

\section{General Procedure for Determination of Kinetic Isotope Effect}

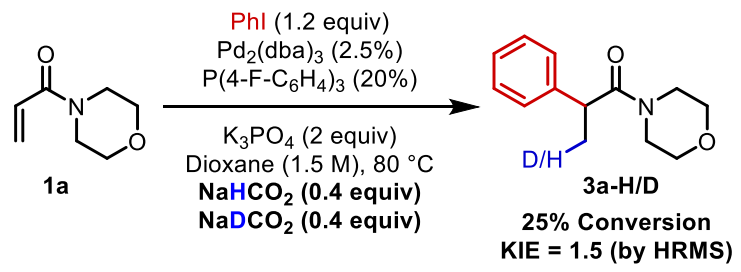

Scheme S9. Determination of kinetic isotope effect 
To a one-dram vial containing a stir bar was added $\mathrm{K}_{3} \mathrm{PO}_{4}(43 \mathrm{mg}, 0.20 \mathrm{mmol})$, sodium formate $(2.72 \mathrm{mg}$, $0.040 \mathrm{mmol})$, sodium formate-D $(2.73 \mathrm{mg}, 0.040 \mathrm{mmol}), \mathrm{TBABF}_{4}(16.5 \mathrm{mg}, 0.050 \mathrm{mmol})$, and 1,3,5trimethoxybenzene $(5.6 \mathrm{mg}, 0.033 \mathrm{mmol})$. Using a micropipette, aryl iodide $(0.140 \mathrm{mmol})$, 4acryloylmorpholine $(14.1 \mathrm{mg}, 12.6 \mu \mathrm{L}, 0.100 \mathrm{mmol})$, tetramethylammonium formate $(30 \mathrm{wt} \%, 53 \mu \mathrm{L}, 0.150$ $\mathrm{mmol})$, and $\mathrm{Pd} /$ Ligand stock solution $(66.5 \mu \mathrm{L})$ was added sequentially to the vial. The vial was sealed and placed in a pre-heated heating block at $80{ }^{\circ} \mathrm{C}$ for 20 minutes. After 20 minutes, the vial was cooled to room temperature and the contents were dissolved in $\mathrm{CDCl}_{3}$ for ${ }^{1} \mathrm{H}$ NMR analysis $(600 \mathrm{MHz})$. The NMR sample was recombined with the reaction mixture, and the solution was loaded directly onto a preparative TLC plate $(40 \%$ EtOAc/Hexanes) to yield 3a $(2.7 \mathrm{mg}, 12 \%)$. This was analyzed by high resolution mass spectrometry to determine a KIE of 1.5 .

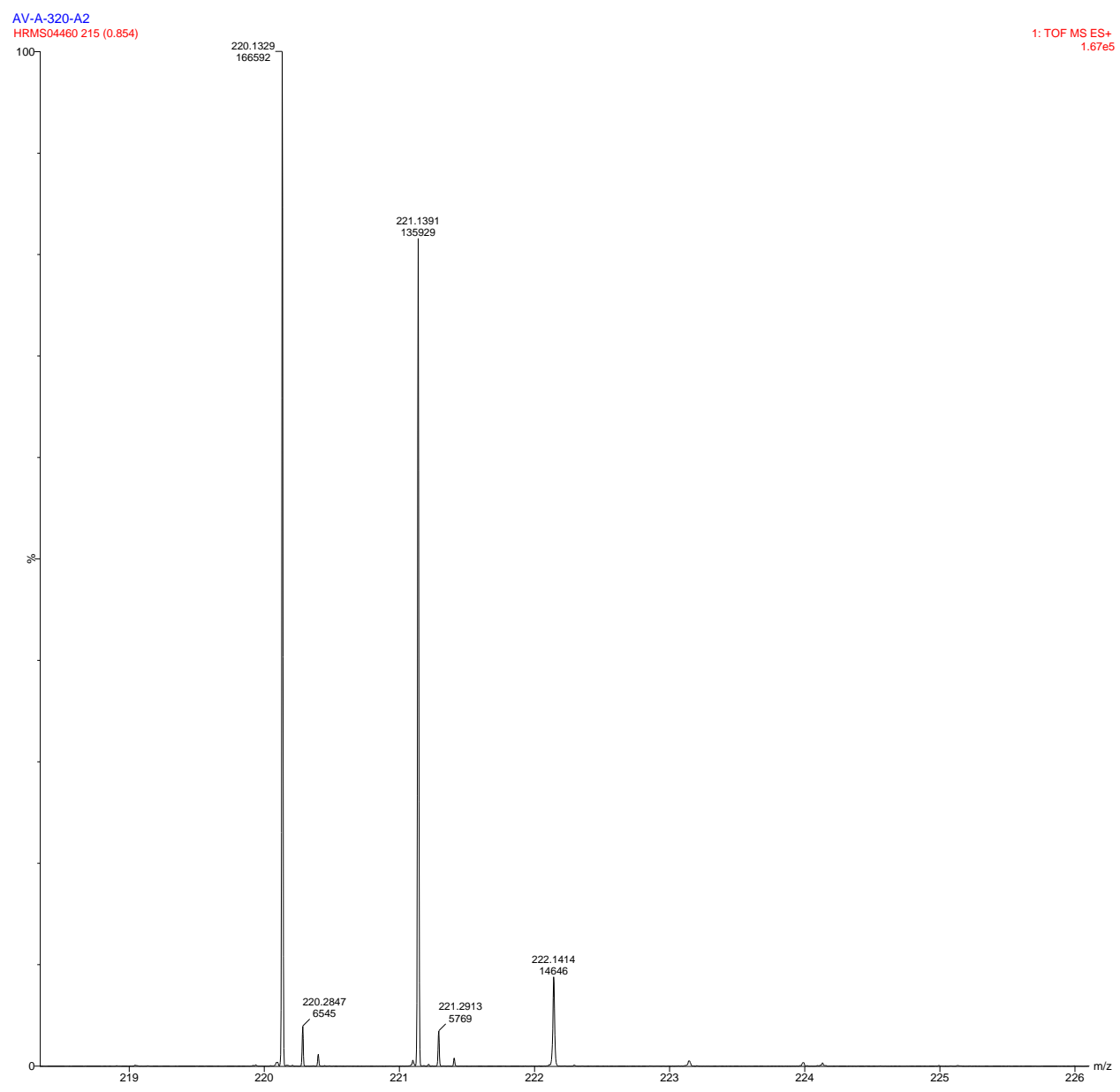

MassWorks 5.0 idealized Gaussian peaks (black) and best fit of 0-2 deuteriums (green). 


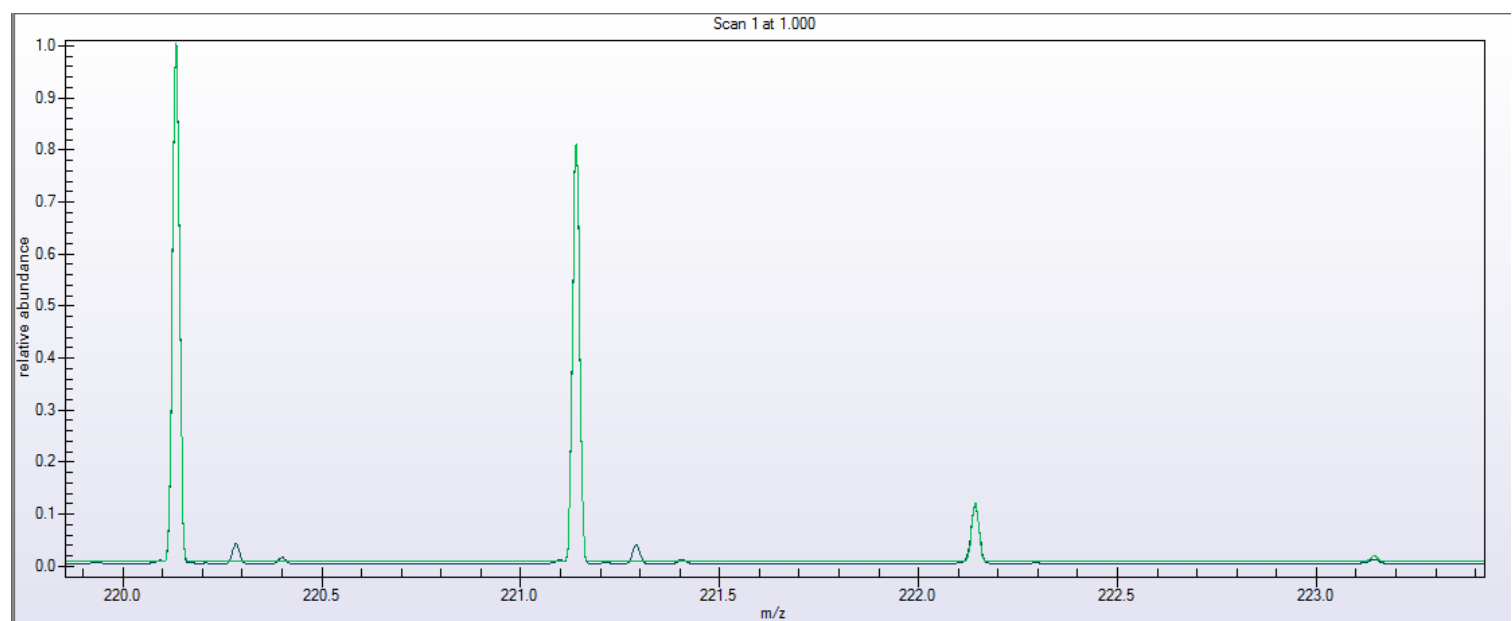

MassWorks calculated mixture composition:

Mixture Results:

$0.598 \mathrm{C}_{13} \mathrm{H}_{18} \mathrm{NO}_{2}$

$0.400 \mathrm{C}_{13} \mathrm{H}_{17} \mathrm{NO}_{2}[2 \mathrm{H}]$

$0.001 \mathrm{C}_{13} \mathrm{H}_{16} \mathrm{NO}_{2}[2 \mathrm{H}]_{2}$

Overall Mass Error (mDa): 0.3822

Figure S6. MassWorks determination of kinetic isotope effect

Evidence of Hydrohalogenation under Standard Reaction Conditions

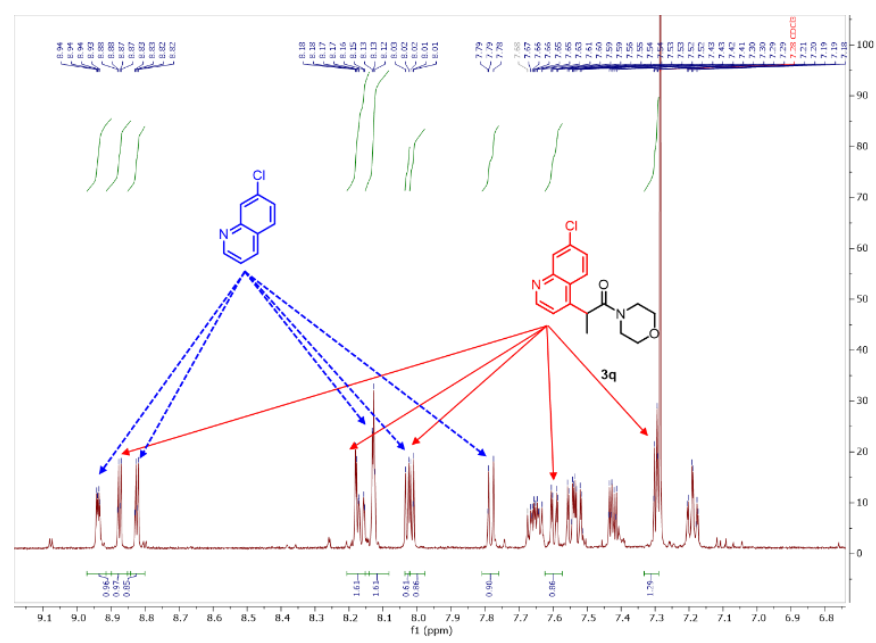

Figure S7: Evidence of hydrodehalogenation in ${ }^{1} \mathrm{H}$ NMR of crude reaction mixture of $\mathbf{3 q}$. 


\section{KINETICS PROCEDURES}

\section{General Procedure for Reaction Kinetics}

All analysis unless otherwise noted is performed via LCMS.

Different Excess:

Pd. Stock: $57 \mathrm{mg} \mathrm{Pd}$ (dba) 3 , $158 \mathrm{mg}$ ligand, and $420 \mathrm{mg}$ 1,3,5-trimethoxybenzene (internal standard, IS) in $1.17 \mathrm{~mL}$ DMF.

Alkene Stock: $315 \mu \mathrm{L}$ alkene in $250 \mu \mathrm{L}$ DMF.

$212 \mathrm{mg}$ of tripotassium phosphate was added to a 2-dram vial followed by alkene stock, iodobenzene, and formate in that order. Pd/ligand/IS stock was added and the vial was then placed in a preheated block. 10 $\mu \mathrm{L}$ aliquots were taken at their respective time points and diluted with $700 \mu \mathrm{L}$ MeCN directly into a LCMS plate.

Standard: $113 \mu \mathrm{L}$ alkene stock, $250 \mu \mathrm{L}$ formate, $67 \mu \mathrm{L}$ PhI, $234 \mu \mathrm{L}$ palladium stock, $100 \mu \mathrm{L}$ DMF

DE-1: $113 \mu \mathrm{L}$ alkene stock, $125 \mu \mathrm{L}$ formate, $100 \mu \mathrm{L}$ DI water, $67 \mu \mathrm{L}$ PhI, $234 \mu \mathrm{L}$ palladium stock, 100 $\mu \mathrm{L} \mathrm{DMF}$

DE-2: $223 \mu \mathrm{L}$ alkene stock, $250 \mu \mathrm{L}$ formate, $67 \mu \mathrm{L}$ PhI, $234 \mu \mathrm{L}$ palladium stock

DE-3: $113 \mu \mathrm{L}$ alkene stock, $250 \mu \mathrm{L}$ formate, $134 \mu \mathrm{L}$ PhI, $234 \mu \mathrm{L}$ palladium stock, $100 \mu \mathrm{L}$ DMF

\section{$\underline{\text { Same Excess: }}$}

Pd. Stock: $45.6 \mathrm{mg} \mathrm{Pd}_{2}(\mathrm{dba})_{3}, 126.4 \mathrm{mg}$ ligand, and $336.4 \mathrm{mg}$ IS in $1.33 \mathrm{~mL} \mathrm{DMF}$.

Standard: $62.9 \mu \mathrm{L}$ alkene (neat), $260 \mu \mathrm{L}$ formate, $66.9 \mu \mathrm{L} \mathrm{PhI,} 332 \mu \mathrm{L}$ stock

SE-1: $41.2 \mu \mathrm{L}$ alkene (neat), $195 \mu \mathrm{L}$ formate, $65 \mu \mathrm{L}$ DI water, $50.2 \mu \mathrm{L}$ PhI, $332 \mu \mathrm{L}$ stock

SE-2: $41.2 \mu \mathrm{L}$ alkene (neat), $195 \mu \mathrm{L}$ formate, $65 \mu \mathrm{L}$ DI water, $50.2 \mu \mathrm{L}$ PhI, $332 \mu \mathrm{L}$ stock , 27.4 mg product

Palladium Order:

A palladium ligand stock solution was prepared using $183.2 \mathrm{mg} \mathrm{Pd} 2(\mathrm{dba})_{3}, 508 \mathrm{mg}$ ligand, and $0.664 \mathrm{~mL}$ of DMF. This was allowed to stir for 5 minutes until dissolved. An acrylamide, iodobenzene, internal standard stock was prepared using $315 \mu \mathrm{L}$ of alkene, $335 \mu \mathrm{L}$ of PhI, and $420 \mathrm{mg}$ of 1,3,5-trimethoxybenzene in $830 \mu \mathrm{L}$ of DMF. $212 \mathrm{mg}$ of tripotassium phosphate was weighed into a 2-dram vial and $296 \mu \mathrm{L}$ of alkene stock was added followed by $260 \mu \mathrm{L}$ of formate. Pd/ligand stock was added followed by DMF to dilute to the proper concentration. The vial was then placed in a preheated block. $5 \mu \mathrm{L}$ aliquots were taken at their respective time points and diluted with $450 \mu \mathrm{L} \mathrm{MeCN}$ directly into a filter vial.

A: to this vial was added $66 \mu \mathrm{L}$ of palladium stock followed by $200 \mu \mathrm{L}$ of DMF to reach palladium concentration of $0.046 \mathrm{M}$ 
B: to this vial was added $166 \mu \mathrm{L}$ of palladium stock followed by $100 \mu \mathrm{L}$ of DMF to reach palladium concentration of $0.116 \mathrm{M}$

C: to this vial was added $266 \mu \mathrm{L}$ of palladium stock to reach palladium concentration of $0.185 \mathrm{M}$

\section{Kinetics Results}

$\underline{\text { Same/Different Excess }}$

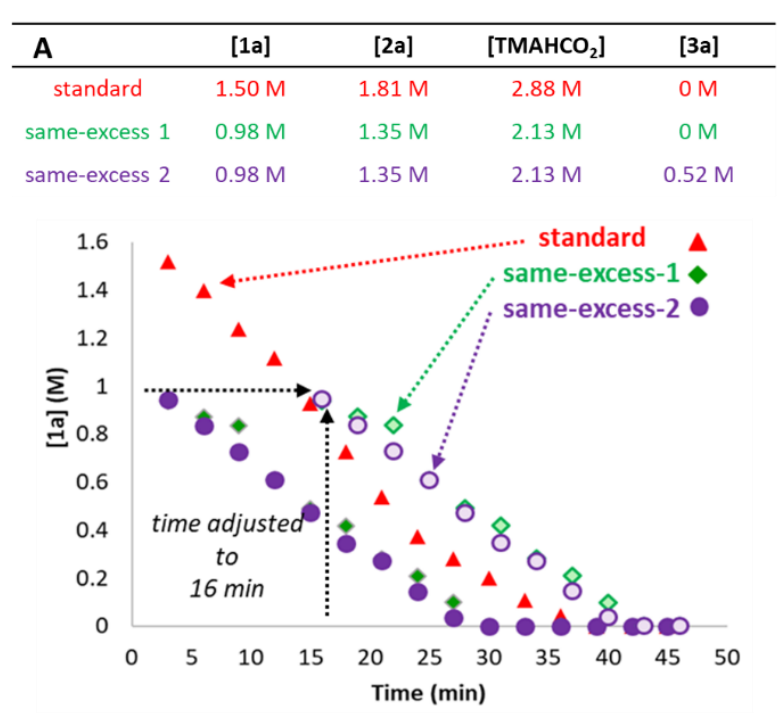

\begin{tabular}{ccccc}
\hline B & [1a] & [2a] & [TMAHCO $]$ & [3a] \\
\hline standard & $1.30 \mathrm{M}$ & $1.56 \mathrm{M}$ & $2.88 \mathrm{M}$ & $0 \mathrm{M}$ \\
diff.-excess 1 & $1.30 \mathrm{M}$ & $1.56 \mathrm{M}$ & $1.44 \mathrm{M}$ & $0 \mathrm{M}$ \\
diff.-excess 2 & $\mathbf{2 . 6 0 \mathrm { M }}$ & $1.56 \mathrm{M}$ & $2.88 \mathrm{M}$ & $0 \mathrm{M}$ \\
diff.-excess 3 & $1.30 \mathrm{M}$ & $\mathbf{3 . 1 2} \mathrm{M}$ & $2.88 \mathrm{M}$ & $0 \mathrm{M}$
\end{tabular}

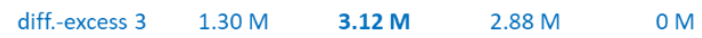

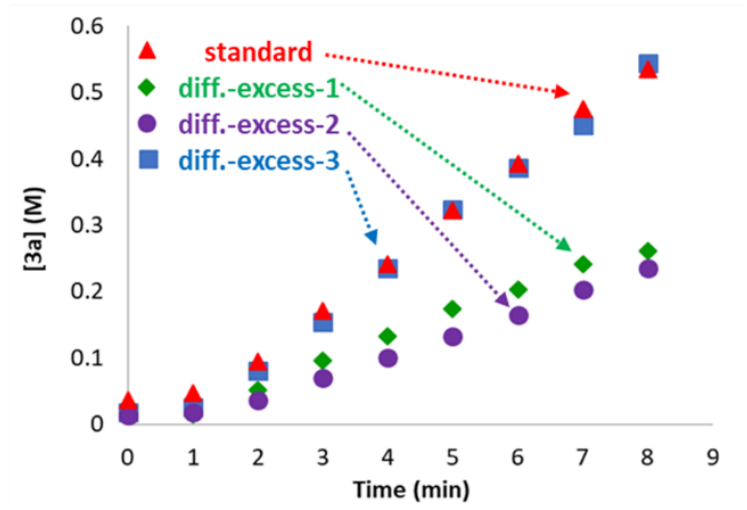

Figure S8. (A) Same excess experiment for reaction shown in eq. 1. (B) Different excess experiments of the reaction shown in eq. 1 .

\begin{tabular}{l|cccc} 
& $\begin{array}{c}\text { [3a] vs } \\
\text { Time }\end{array}$ & $\mathbf{0 . 0 4 6} \mathbf{M}$ & $\mathbf{0 . 1 1 6} \mathbf{M}$ & $\mathbf{0 . 1 8 5} \mathbf{M}$ \\
\cline { 2 - 5 } & $0 \mathrm{~min}$ & $0 \mathrm{M}$ & $0 \mathrm{M}$ & $0 \mathrm{M}$ \\
induction & $1 \mathrm{~min}$ & $0 \mathrm{M}$ & $0.006 \mathrm{M}$ & $0.006 \mathrm{M}$ \\
period & $2 \mathrm{~min}$ & $0.005 \mathrm{M}$ & $0.012 \mathrm{M}$ & $0.018 \mathrm{M}$ \\
& $3 \mathrm{~min}$ & $0.015 \mathrm{M}$ & $0.023 \mathrm{M}$ & $0.074 \mathrm{M}$ \\
& $4 \mathrm{~min}$ & $0.065 \mathrm{M}$ & $0.215 \mathrm{M}$ & $0.145 \mathrm{M}$
\end{tabular}




\begin{tabular}{l|llll}
5 min & $0.048 \mathrm{M}$ & $0.302 \mathrm{M}$ & $0.489 \mathrm{M}$ & Determining Order in Palladium \\
7 min & $0.155 \mathrm{M}$ & $0.492 \mathrm{M}$ & $0.695 \mathrm{M}$ &
\end{tabular}

Figure S9. Determination of order in palladium using initial rates.

$\underline{\text { Full Reaction Profile }}$

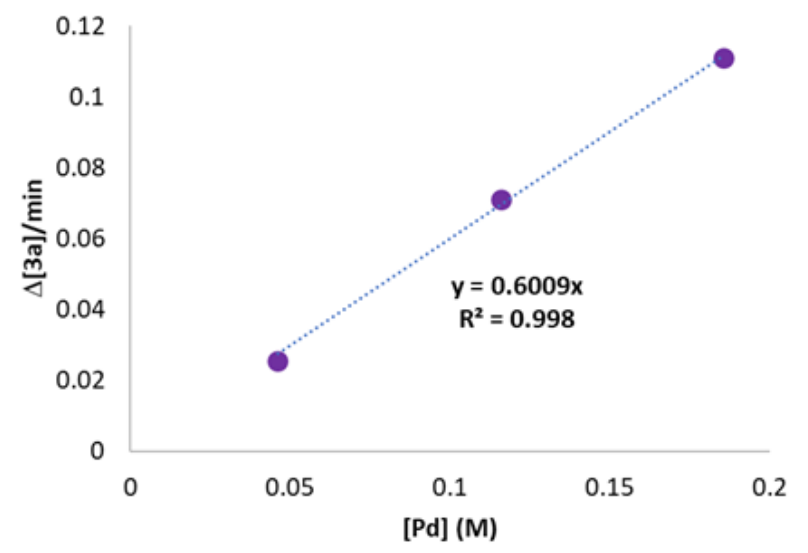




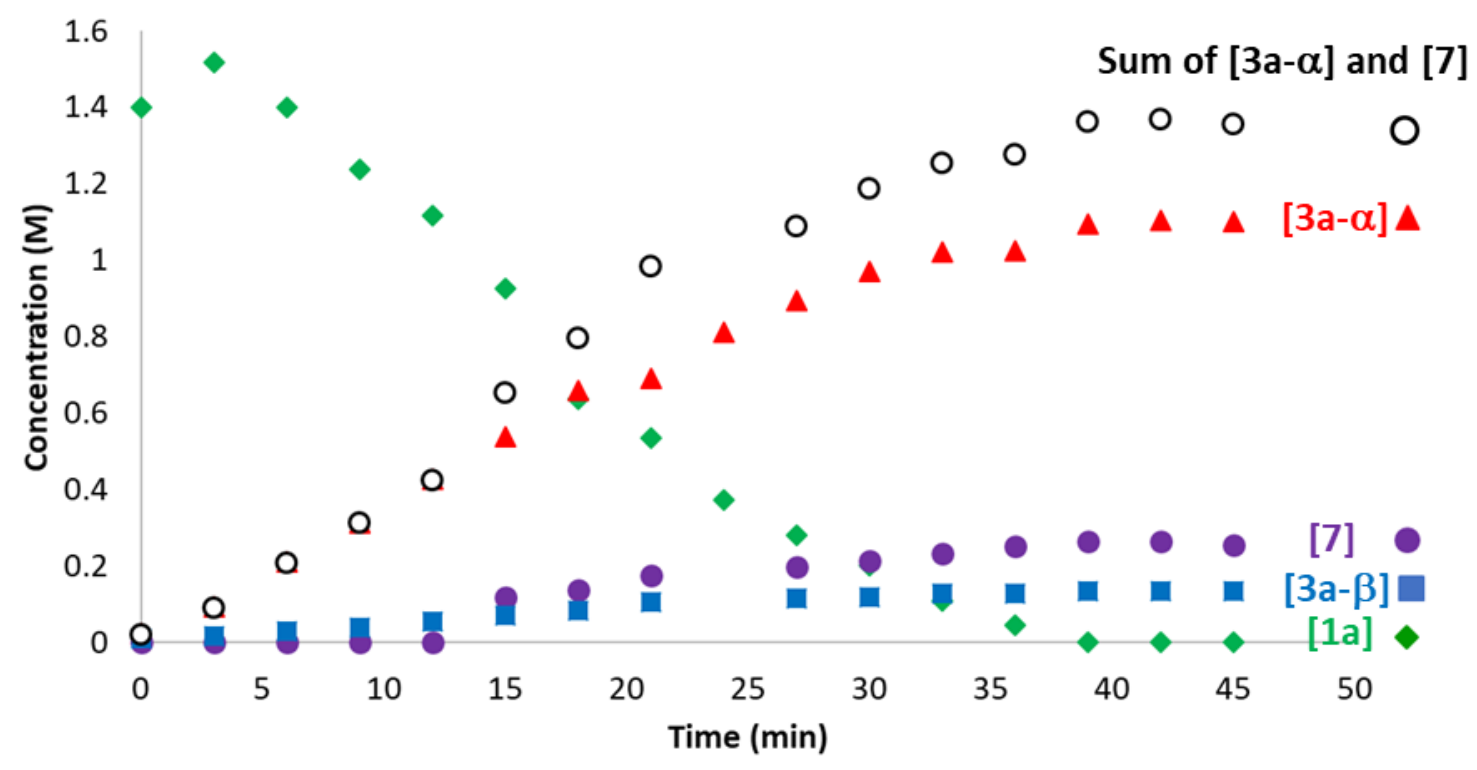

Figure S10. Graphical representation the speciation during the course of the reaction.

\section{Ligand Loadings}

Kinetics performed followed Palladium Order general procedure with the exception that palladium, ligand, and base are weighed individually into each vial, allowed to premix, and then charged with alkene/ArI/IS stock, and formate.

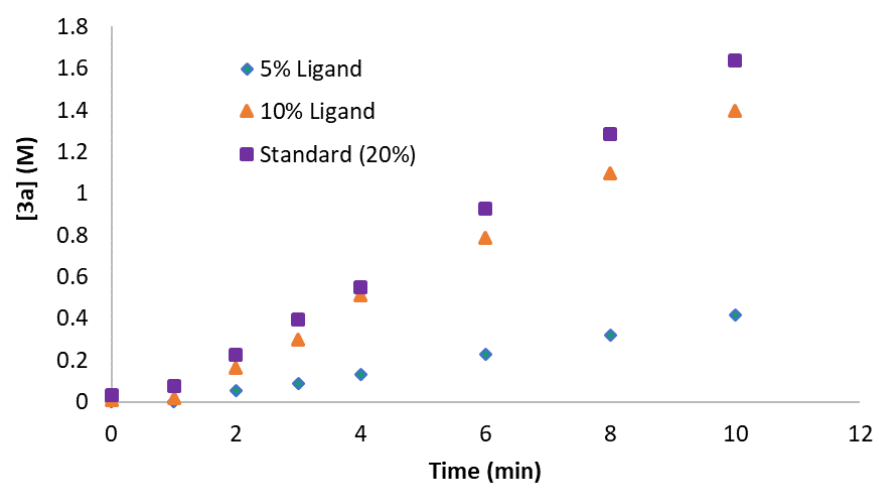

Figure S11. Different excess experiment varying ligand loadings.

\section{In Situ NMR Protocol}

To an NMR tube was added 4-acryloylmorpholine $(62.9 \mu \mathrm{L}, 0.500 \mathrm{mmol})$, iodobenzene $(66.9 \mu \mathrm{L}, 0.600$ $\mathrm{mmol})$, tetramethylammonium formate $(260 \mu \mathrm{L}, 30 \mathrm{wt} \%, 0.750 \mathrm{mmol})$, tripotassium phosphate $(212 \mathrm{mg}$, $1.00 \mathrm{mmol}$ ), and $400 \mu \mathrm{L}$ of DMF-d7. This was placed in a $400 \mathrm{MHz}$ spectrometer and brought to $70{ }^{\circ} \mathrm{C}$. Once at temperature, the sample was locked and shimmed. To this warm NMR tube was added premixed 
$\mathrm{Pd}_{2}(\mathrm{dba})_{3}(11.4 \mathrm{mg}, 0.0125 \mathrm{mmol})$ and triphenylphosphine $(26.2 \mathrm{mg}, 0.100 \mathrm{mmol})$ in $100 \mu \mathrm{L} \mathrm{DMF}-\mathrm{d} 7$. This was placed back in the spectrometer and spectra was collected every two minutes for 18 minutes.
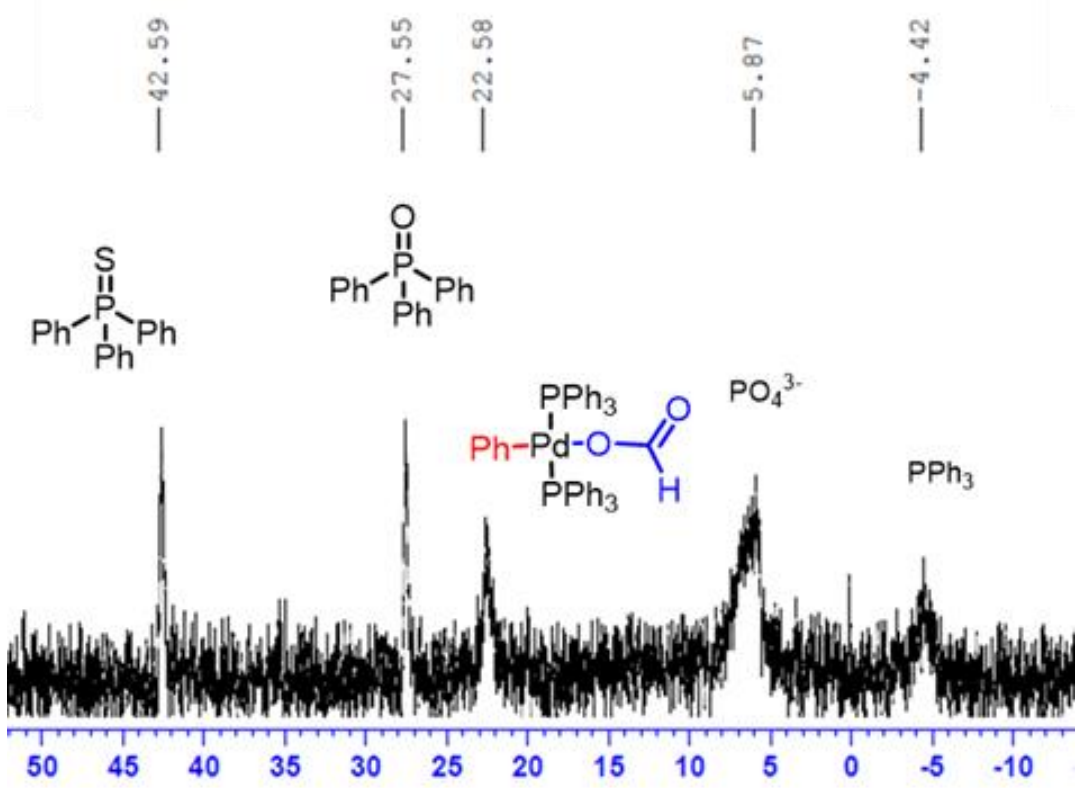

Figure S12. In situ NMR spectrum.

\section{NMR Control Experiments}

To an NMR tube was added 4-acryloylmorpholine $(12.6 \mu \mathrm{L}, 0.100 \mathrm{mmol})$, iodobenzene $(13.4 \mu \mathrm{L}, 0.120$ $\mathrm{mmol}$ ), 8 (106 mg, $0.110 \mathrm{mmol}$ ), and $500 \mu \mathrm{L}$ of DMF-d7. ${ }^{19} \mathrm{~F}$ NMR spectra was collected (spectra A). To this NNMR tube was added $100 \mu \mathrm{L}$ of aq. TMA $\bullet \mathrm{HCO}_{2}$ and an NMR spectra was collected (spectra B).

This was compared to an in-situ measurement following the in situ NMR Protocol using tris(4fluorophenyl)phosphine as ligand (spectra C).

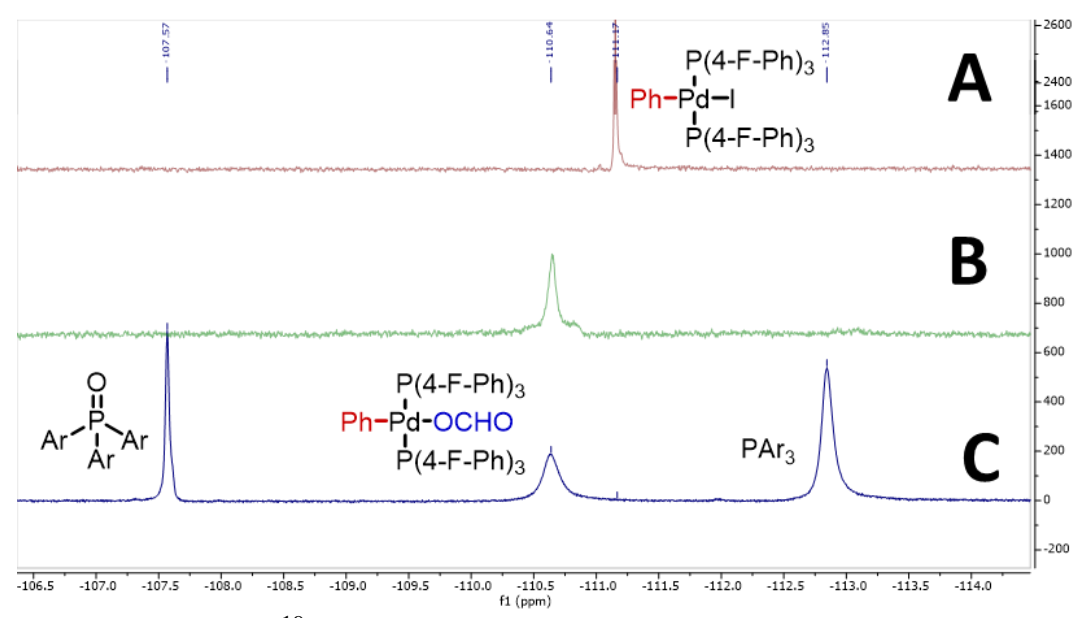

Figure S13: (A) ${ }^{19}$ F NMR spectra of complex 8, 1a, and 2a in DMF-d7. (B) NMR spectra resulting from adding aq. formate to the mixture in A. (C) in situ NMR spectra of the reaction under standard conditions using tris(4-fluorophenyl)phosphine as ligand. 


\section{REFERENCES:}

1. Eriksson, J.; Åberg, O.; Långström, B. Synthesis of [11C]/[13C]Acrylamides by Palladium-Mediated Carbonylation. Eur. J. Org. Chem. 2007, 455-461.

2. Ikoma, M.; Oikawa, M.; Sasaki, M. Synthesis and Domino Metathesis of Functionalized 7Oxanorbornene Analogs toward cis-Fused Heterocycles. Tetrahedron 2008, 64, 2740-2749.

3. Abarca, B.; Custodio, R.; Cuadro, A. M.; Sucunza, D.; Domingo, A.; Mendicuti, F.; Alvarez-Builla, J.; Vaquero, J. J. Efficient Synthesis of an Indoloquinolizinium Alkaloid Selective DNA-Binder by RingClosing Metathesis. Org. Lett. 2014, 16, 3464-3467.

4. Rodríguez-Fernández, M.; Yan, X.; Collados, J. F.; White, P. B.; Harutyunyan, S. R. Lewis Acid Enabled Copper-Catalyzed Asymmetric Synthesis of Chiral $\beta$-Substituted Amides. J. Am. Chem. Soc 2017, 139, 14224-14231.

5. Wang, Z.-X.; Bai, X.-Y.; Yao, H.-C.; Li, B.-J, Synthesis of Amides with Remote Stereocenters by Catalytic Asymmetric $\gamma$-Alkynylation of $\alpha, \beta$-Unsaturated Amides. J. Am. Chem. Soc. 2016, 138, 1487214875

6. Grzyb, J. A.; Shen, M.; Yoshina-Ishii, C.; Chi, W.; Brown, R. S.; Batey, R. A. Carbamoylimidazolium and Thiocarbamoylimidazolium Salts: Novel Reagents for the Synthesis of Ureas, Thioureas, Carbamates, Thiocarbamates and Amides. Tetrahedron 2005, 61, 7153-7175.

7. Ito, K.; Iida, T.; Fujita, T.; Tsuji, S. A Simple Method for the Synthesis of Amides by Use of 2,2'Dibenzothiazolyl Disulfide as an Oxidant. Synthesis 1981, 287-287.

8. Besset, T.; Cahard, D.; Pannecoucke, X. Regio- and Diastereoselective Cu-Mediated Trifluoromethylation of Functionalized Alkenes. J. Org. Chem. 2014, 79, 413-418.

9. Grzyb, J. A.; Batey, R. A. Achieving Functional Group Diversity in Parallel Synthesis: Solution-Phase Synthesis of a Library of Ureas, Carbamates, Thiocarbamates, and Amides Using Carbamoylimidazolium Salts. Tetrahedron Lett. 2008, 49, 5279-5282.

10. Zhang, X. K.; Chuang, W.-L.; Elbin, C. S.; Scheidegger, D.; Beauregard, C.; Pickering, S.; Pacheco, J.; Keutzer, J. Assay Mixtures for Determining Lysosomal Enzymes and Uses for Screening for Lysosomal Storage Disease. U.S. Patent WO2008033427, March 20, 2008.

11. Gholinejad, M.; Dasvarz, N.; Nájera, C. Novel Oxime-Palladacycle Supported on Clay Composite as an Efficient Heterogeneous Catalyst for Sonogashira Reaction. Inorg. Chim. Acta 2018, 483, 262-270.

12. Sinha, M.; Dola, V. R.; Agarwal, P.; Srivastava, K.; Haq, W.; Puri, S. K.; Katti, S. B. Antiplasmodial Activity of New 4-Aminoquinoline Derivatives Against Chloroquine Resistant Strain. Bioorg. Med. Chem. 2014, 22, 3573-3586.

13. CCDC 1987658 (8) contains the supplementary crystallographic data for this paper. This data can be obtained free of charge from The Cambridge Crystallographic Data Centre via www.ccdc.cam.ac.uk/data_request/cif.

14. Zhu, J.; Gao, B.; Huang, H. Palladium-Catalyzed Highly Regioselective Hydroaminocarbonylation of Aromatic Alkenes to Branched Amides. Org. Biomol. Chem. 2017, 15, 2910-2913.

15. Hama, T.; Culkin, D. A.; Hartwig, J. F. Palladium-Catalyzed Intermolecular $\alpha$-Arylation of Zinc Amide Enolates under Mild Conditions. J. Am. Chem. Soc. 2006, 128, 4976-4985.

16. Vedejs, E.; Kruger, A. W.; Lee, N.; Sakata, S. T.; Stec, M.; Suna, E. Enantioselective Enolate Protonation with Chiral Anilines: Scope, Structural Requirements, and Mechanistic Implications. J. Am. Chem. Soc. 2000, 122, 4602-4607.

17. Ye, W.; Mo, J.; Zhao, T.; Xu, B. Palladium-Catalyzed Amidation-Hydrolysis Reaction of gemDihaloolefins: Efficient Synthesis of Homologated Carboxamides from Ketones. Chem. Commun. 2009, 3246-3248.

18. van Leeuwen, M.; McKillop, A. A Study of the Ferrous Ion-Initiated $S_{\mathrm{RN}} 1$ Reactions of Halogenoarenes with tert-Butyl Acetate and N-AcylmorpholineEnolates. J. Chem. Soc., Perkin Trans. 1 1993, 2433-2440. 


\section{X-RAY CRYSTALLOGRAPHY}

\section{Experimental Summary for Crystal_8 (CCDC 1987658)}

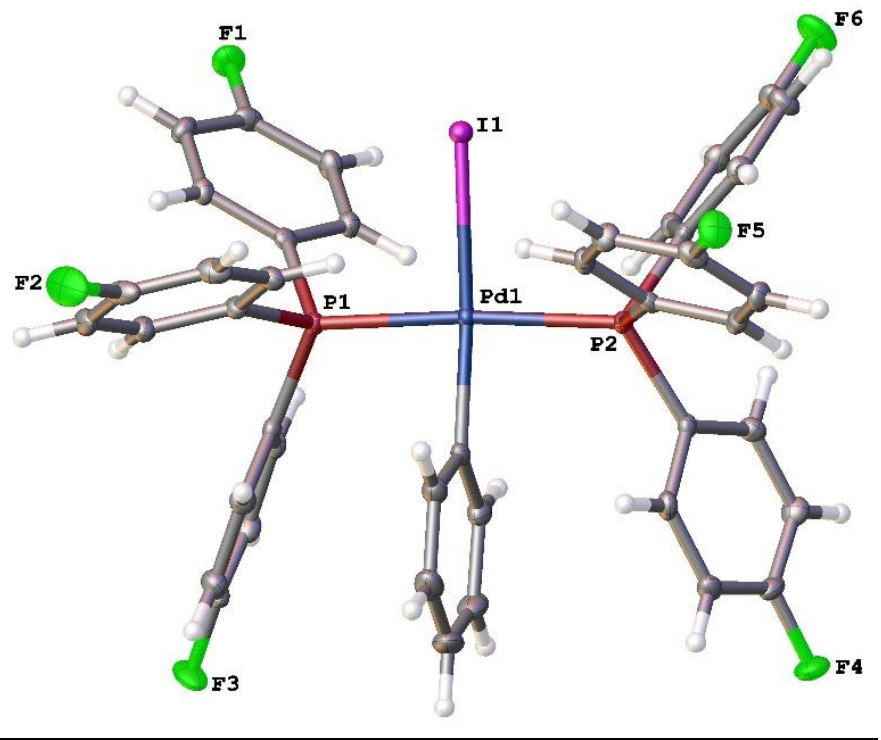

Figure S14. Single crystal X-ray structure of 8

The single crystal X-ray diffraction studies were carried out on a Bruker APEX II ULTRA CCD diffractometer equipped with $\mathrm{Mo} \mathrm{K}_{\alpha}$ radiation $(\lambda=0.71073 \AA)$. Crystals of the subject compound were used as received (grown from $\mathrm{DCM} / \mathrm{Et}_{2} \mathrm{O}$ ). A 0.170 × $0.060 \times 0.050 \mathrm{~mm}$ colorless block crystal was mounted on a Cryoloop with Paratone $\mathrm{N}$ oil.

Data were collected in a nitrogen gas stream at 100(2) K using $\varpi$ scans. Crystal-to-detector distance was $45 \mathrm{~mm}$ using an exposure time of 1.0 second with a scan width of $0.65^{\circ}$. Data collection was $100.0 \%$ complete to $25.242^{\circ}$ in $\theta$. A total of 32138 reflections were collected. 7932 reflections were found to be symmetry independent, with an $\mathrm{R}_{\text {int }}$ of 0.0419 . Indexing and unit cell refinement indicated a Primitive Monoclinic lattice. The space group was found to be $P 2_{l} / c$. The data were integrated using the Bruker SAINT Software program and scaled using the SADABS software program. Solution by direct methods (SHELXT) produced a complete phasing model consistent with the proposed structure. 
All non-hydrogen atoms were refined anisotropically by full-matrix least-squares (SHELXL-2014).

All carbon bonded hydrogen atoms were placed using a riding model. Their positions were constrained relative to their parent atom using the appropriate HFIX command in SHELXL-2014.

Notes: Excellent data and refinement. The crystal lattice contains one molecule of $\mathrm{CH}_{2} \mathrm{Cl}_{2}$ (disordered) per formula unit.

Table S2. Crystal data and structure refinement for Engle213.

Report date

Identification code

Empirical formula

Formula weight

Temperature

Wavelength

Crystal system

Space group

Unit cell dimensions

Volume

$\mathrm{Z}$

Density (calculated)

Absorption coefficient

$\mathrm{F}(000)$

Crystal size

Theta range for data collection

Index ranges

Reflections collected

Independent reflections

Completeness to theta $=25.242^{\circ}$

Absorption correction

Max. and min. transmission

Refinement method
2019-10-03

Engle213

C43 H31 Cl2 F6 I P2 Pd

1027.82

$100.0 \mathrm{~K}$

$0.71073 \AA$

Monoclinic

P 1 21/c 1

$\mathrm{a}=14.9867(6) \AA \quad \alpha=90^{\circ}$.

$\mathrm{b}=11.4710(5) \AA \quad \beta=104.3650(10)^{\circ}$.

$\mathrm{c}=24.2026(10) \AA \quad \gamma=90^{\circ}$.

4030.6(3) $\AA^{3}$

4

$1.694 \mathrm{Mg} / \mathrm{m}^{3}$

$1.497 \mathrm{~mm}^{-1}$

2024

$0.17 \times 0.06 \times 0.05 \mathrm{~mm}^{3}$

1.737 to $26.021^{\circ}$.

$-18<=\mathrm{h}<=18,-14<=\mathrm{k}<=14,-29<=\mathrm{l}<=29$

32138

$7932[\mathrm{R}($ int $)=0.0419]$

$100.0 \%$

Semi-empirical from equivalents

0.4908 and 0.4379

Full-matrix least-squares on $\mathrm{F}^{2}$ 
Data / restraints / parameters

Goodness-of-fit on $\mathrm{F}^{2}$

Final R indices [I $>2 \operatorname{sigma}(\mathrm{I})]$

$\mathrm{R}$ indices (all data)

Extinction coefficient

Largest diff. peak and hole
7932 / 2 / 503

1.030

$\mathrm{R} 1=0.0239, \mathrm{wR} 2=0.0541$

$\mathrm{R} 1=0.0288, \mathrm{wR} 2=0.0565$

$\mathrm{n} / \mathrm{a}$

0.475 and -0.338 e. $\AA^{-3}$ 
Table S3. Atomic coordinates ( x 104) and equivalent isotropic displacement parameters $\left(\AA^{2} \times 10^{3}\right)$ for Engle213. U(eq) is defined as one third of the trace of the orthogonalized $U^{i j}$ tensor.

\begin{tabular}{|c|c|c|c|c|}
\hline & $\mathrm{x}$ & $\mathrm{y}$ & $\mathrm{z}$ & $\mathrm{U}(\mathrm{eq})$ \\
\hline $\mathrm{I}(1)$ & $4142(1)$ & $3606(1)$ & $2402(1)$ & $15(1)$ \\
\hline $\operatorname{Pd}(1)$ & $2784(1)$ & $5052(1)$ & $2543(1)$ & $10(1)$ \\
\hline $\mathrm{P}(1)$ & $3264(1)$ & 4899(1) & $3527(1)$ & $11(1)$ \\
\hline $\mathrm{P}(2)$ & 2271(1) & $5408(1)$ & $1568(1)$ & $11(1)$ \\
\hline $\mathrm{F}(1)$ & $7290(1)$ & $5593(1)$ & $4397(1)$ & $25(1)$ \\
\hline $\mathrm{F}(2)$ & 1951(1) & $473(1)$ & $4308(1)$ & $38(1)$ \\
\hline $\mathrm{F}(3)$ & $1739(1)$ & $8403(1)$ & $4850(1)$ & $28(1)$ \\
\hline $\mathrm{F}(4)$ & $-118(1)$ & $9565(1)$ & 932(1) & $25(1)$ \\
\hline $\mathrm{F}(5)$ & $448(1)$ & $1405(1)$ & 204(1) & $24(1)$ \\
\hline $\mathrm{F}(6)$ & $5494(1)$ & $6225(1)$ & $609(1)$ & $32(1)$ \\
\hline $\mathrm{C}(1)$ & $4508(2)$ & $5036(2)$ & $3798(1)$ & $13(1)$ \\
\hline$C(2)$ & $5023(2)$ & $4367(2)$ & $4244(1)$ & $15(1)$ \\
\hline$C(3)$ & $5967(2)$ & $4541(2)$ & $4442(1)$ & $18(1)$ \\
\hline$C(4)$ & $6370(2)$ & $5399(2)$ & 4194(1) & $17(1)$ \\
\hline$C(5)$ & $5891(2)$ & $6087(2)$ & $3752(1)$ & $18(1)$ \\
\hline$C(6)$ & $4955(2)$ & $5884(2)$ & $3550(1)$ & $16(1)$ \\
\hline$C(7)$ & $2915(2)$ & $3518(2)$ & $3780(1)$ & $14(1)$ \\
\hline$C(8)$ & $2558(2)$ & $2642(2)$ & $3388(1)$ & $16(1)$ \\
\hline $\mathrm{C}(9)$ & $2235(2)$ & $1608(2)$ & $3564(1)$ & $22(1)$ \\
\hline$C(10)$ & $2277(2)$ & $1473(2)$ & $4137(1)$ & $24(1)$ \\
\hline
\end{tabular}




\begin{tabular}{|c|c|c|c|c|}
\hline $\mathrm{C}(11)$ & $2616(2)$ & $2316(2)$ & $4534(1)$ & $23(1)$ \\
\hline $\mathrm{C}(12)$ & $2936(2)$ & $3339(2)$ & $4354(1)$ & $18(1)$ \\
\hline $\mathrm{C}(13)$ & $2832(2)$ & $5959(2)$ & $3958(1)$ & $13(1)$ \\
\hline$C(14)$ & $3304(2)$ & $6996(2)$ & $4142(1)$ & $16(1)$ \\
\hline$C(15)$ & $2937(2)$ & $7822(2)$ & $4439(1)$ & $20(1)$ \\
\hline$C(16)$ & 2098(2) & $7608(2)$ & $4553(1)$ & 19(1) \\
\hline $\mathrm{C}(17)$ & $1610(2)$ & $6603(2)$ & $4380(1)$ & $20(1)$ \\
\hline $\mathrm{C}(18)$ & $1978(2)$ & $5789(2)$ & $4078(1)$ & $17(1)$ \\
\hline$C(19)$ & $1533(2)$ & $6671(2)$ & $1328(1)$ & $12(1)$ \\
\hline$C(20)$ & $645(2)$ & $6673(2)$ & $1414(1)$ & $17(1)$ \\
\hline$C(21)$ & $82(2)$ & $7649(2)$ & $1279(1)$ & $18(1)$ \\
\hline $\mathrm{C}(22)$ & $422(2)$ & $8603(2)$ & $1056(1)$ & $17(1)$ \\
\hline$C(23)$ & $1275(2)$ & $8626(2)$ & $949(1)$ & $19(1)$ \\
\hline $\mathrm{C}(24)$ & $1835(2)$ & $7652(2)$ & 1089(1) & $16(1)$ \\
\hline$C(25)$ & $1636(2)$ & $4207(2)$ & $1160(1)$ & $12(1)$ \\
\hline$C(26)$ & $1134(2)$ & $4367(2)$ & $595(1)$ & $16(1)$ \\
\hline$C(27)$ & $724(2)$ & $3423(2)$ & $272(1)$ & $18(1)$ \\
\hline $\mathrm{C}(28)$ & $827(2)$ & $2335(2)$ & $521(1)$ & $18(1)$ \\
\hline $\mathrm{C}(29)$ & $1296(2)$ & $2150(2)$ & $1078(1)$ & $18(1)$ \\
\hline $\mathrm{C}(30)$ & 1711(2) & $3099(2)$ & $1397(1)$ & $15(1)$ \\
\hline $\mathrm{C}(31)$ & $3246(2)$ & $5641(2)$ & $1258(1)$ & $13(1)$ \\
\hline $\mathrm{C}(32)$ & $3918(2)$ & $6453(2)$ & $1512(1)$ & $14(1)$ \\
\hline $\mathrm{C}(33)$ & $4668(2)$ & $6665(2)$ & 1291(1) & $17(1)$ \\
\hline $\mathrm{C}(34)$ & $4753(2)$ & $6018(2)$ & $827(1)$ & 19(1) \\
\hline$C(35)$ & $4129(2)$ & $5185(2)$ & $575(1)$ & $20(1)$ \\
\hline
\end{tabular}




\begin{tabular}{lrrrr}
$\mathrm{C}(36)$ & $3364(2)$ & $5004(2)$ & $794(1)$ & $17(1)$ \\
$\mathrm{C}(37)$ & $1662(2)$ & $5876(2)$ & $2668(1)$ & $14(1)$ \\
$\mathrm{C}(38)$ & $895(2)$ & $5205(2)$ & $2683(1)$ & $18(1)$ \\
$\mathrm{C}(39)$ & $103(2)$ & $5735(3)$ & $2771(1)$ & $27(1)$ \\
$\mathrm{C}(40)$ & $76(2)$ & $6923(3)$ & $2848(1)$ & $34(1)$ \\
$\mathrm{C}(41)$ & $840(2)$ & $7588(2)$ & $2838(1)$ & $29(1)$ \\
$\mathrm{C}(42)$ & $1632(2)$ & $7072(2)$ & $2746(1)$ & $19(1)$ \\
$\mathrm{C}(43)$ & $3628(2)$ & $10182(2)$ & $2694(1)$ & $26(1)$ \\
$\mathrm{Cl}(1)$ & $3638(5)$ & $9253(5)$ & $3276(2)$ & $27(1)$ \\
$\mathrm{Cl}(2)$ & $2536(3)$ & $10198(5)$ & $2192(1)$ & $37(1)$ \\
$\mathrm{Cl}(1 \mathrm{~A})$ & $3622(14)$ & $9089(11)$ & $3205(5)$ & $27(1)$ \\
$\mathrm{Cl}(2 \mathrm{~A})$ & $2675(5)$ & $9890(8)$ & $2130(3)$ & $37(1)$ \\
& & & & \\
\hline
\end{tabular}


Table S4. Bond lengths $[\AA]$ and angles $\left[{ }^{\circ}\right]$ for Engle 213 .

\begin{tabular}{ll}
\hline $\mathrm{I}(1)-\mathrm{Pd}(1)$ & $2.7114(2)$ \\
$\mathrm{Pd}(1)-\mathrm{P}(1)$ & $2.3175(6)$ \\
$\mathrm{Pd}(1)-\mathrm{P}(2)$ & $2.3302(6)$ \\
$\mathrm{Pd}(1)-\mathrm{C}(37)$ & $2.016(2)$ \\
$\mathrm{P}(1)-\mathrm{C}(1)$ & $1.824(2)$ \\
$\mathrm{P}(1)-\mathrm{C}(7)$ & $1.821(2)$ \\
$\mathrm{P}(1)-\mathrm{C}(13)$ & $1.823(2)$ \\
$\mathrm{P}(2)-\mathrm{C}(19)$ & $1.828(2)$ \\
$\mathrm{P}(2)-\mathrm{C}(25)$ & $1.820(2)$ \\
$\mathrm{P}(2)-\mathrm{C}(31)$ & $1.378(3)$ \\
$\mathrm{F}(1)-\mathrm{C}(4)$ & $1.369(2)$ \\
$\mathrm{F}(2)-\mathrm{C}(10)$ & $1.363(3)$ \\
$\mathrm{F}(3)-\mathrm{C}(16)$ & $1.35(3)$ \\
$\mathrm{F}(4)-\mathrm{C}(22)$ & $1.35(3)$ \\
$\mathrm{F}(5)-\mathrm{C}(28)$ & \\
$\mathrm{C}(6)-\mathrm{C}(34)$ & $1.35(3)$ \\
\hline
\end{tabular}




\begin{tabular}{|c|c|}
\hline $\mathrm{C}(5)-\mathrm{H}(5)$ & 0.9500 \\
\hline$C(5)-C(6)$ & $1.386(3)$ \\
\hline $\mathrm{C}(6)-\mathrm{H}(6)$ & 0.9500 \\
\hline$C(7)-C(8)$ & $1.394(3)$ \\
\hline$C(7)-C(12)$ & $1.399(3)$ \\
\hline $\mathrm{C}(8)-\mathrm{H}(8)$ & 0.9500 \\
\hline$C(8)-C(9)$ & $1.387(3)$ \\
\hline $\mathrm{C}(9)-\mathrm{H}(9)$ & 0.9500 \\
\hline$C(9)-C(10)$ & $1.381(4)$ \\
\hline$C(10)-C(11)$ & $1.370(4)$ \\
\hline $\mathrm{C}(11)-\mathrm{H}(11)$ & 0.9500 \\
\hline$C(11)-C(12)$ & $1.378(3)$ \\
\hline $\mathrm{C}(12)-\mathrm{H}(12)$ & 0.9500 \\
\hline$C(13)-C(14)$ & $1.400(3)$ \\
\hline$C(13)-C(18)$ & $1.393(3)$ \\
\hline $\mathrm{C}(14)-\mathrm{H}(14)$ & 0.9500 \\
\hline$C(14)-C(15)$ & $1.383(3)$ \\
\hline $\mathrm{C}(15)-\mathrm{H}(15)$ & 0.9500 \\
\hline$C(15)-C(16)$ & $1.374(3)$ \\
\hline $\mathrm{C}(16)-\mathrm{C}(17)$ & $1.374(3)$ \\
\hline $\mathrm{C}(17)-\mathrm{H}(17)$ & 0.9500 \\
\hline$C(17)-C(18)$ & $1.383(3)$ \\
\hline $\mathrm{C}(18)-\mathrm{H}(18)$ & 0.9500 \\
\hline $\mathrm{C}(19)-\mathrm{C}(20)$ & $1.397(3)$ \\
\hline $\mathrm{C}(19)-\mathrm{C}(24)$ & $1.391(3)$ \\
\hline
\end{tabular}




\begin{tabular}{|c|c|}
\hline $\mathrm{C}(20)-\mathrm{H}(20)$ & 0.9500 \\
\hline $\mathrm{C}(20)-\mathrm{C}(21)$ & $1.391(3)$ \\
\hline $\mathrm{C}(21)-\mathrm{H}(21)$ & 0.9500 \\
\hline $\mathrm{C}(21)-\mathrm{C}(22)$ & $1.374(3)$ \\
\hline$C(22)-C(23)$ & $1.365(3)$ \\
\hline $\mathrm{C}(23)-\mathrm{H}(23)$ & 0.9500 \\
\hline $\mathrm{C}(23)-\mathrm{C}(24)$ & $1.388(3)$ \\
\hline $\mathrm{C}(24)-\mathrm{H}(24)$ & 0.9500 \\
\hline$C(25)-C(26)$ & $1.399(3)$ \\
\hline$C(25)-C(30)$ & $1.388(3)$ \\
\hline $\mathrm{C}(26)-\mathrm{H}(26)$ & 0.9500 \\
\hline$C(26)-C(27)$ & $1.387(3)$ \\
\hline $\mathrm{C}(27)-\mathrm{H}(27)$ & 0.9500 \\
\hline$C(27)-C(28)$ & $1.378(3)$ \\
\hline $\mathrm{C}(28)-\mathrm{C}(29)$ & $1.373(3)$ \\
\hline $\mathrm{C}(29)-\mathrm{H}(29)$ & 0.9500 \\
\hline $\mathrm{C}(29)-\mathrm{C}(30)$ & $1.389(3)$ \\
\hline $\mathrm{C}(30)-\mathrm{H}(30)$ & 0.9500 \\
\hline $\mathrm{C}(31)-\mathrm{C}(32)$ & $1.397(3)$ \\
\hline $\mathrm{C}(31)-\mathrm{C}(36)$ & $1.389(3)$ \\
\hline $\mathrm{C}(32)-\mathrm{H}(32)$ & 0.9500 \\
\hline $\mathrm{C}(32)-\mathrm{C}(33)$ & $1.381(3)$ \\
\hline $\mathrm{C}(33)-\mathrm{H}(33)$ & 0.9500 \\
\hline $\mathrm{C}(33)-\mathrm{C}(34)$ & $1.378(3)$ \\
\hline $\mathrm{C}(34)-\mathrm{C}(35)$ & $1.371(3)$ \\
\hline
\end{tabular}




\begin{tabular}{|c|c|}
\hline $\mathrm{C}(35)-\mathrm{H}(35)$ & 0.9500 \\
\hline$C(35)-C(36)$ & $1.392(3)$ \\
\hline $\mathrm{C}(36)-\mathrm{H}(36)$ & 0.9500 \\
\hline $\mathrm{C}(37)-\mathrm{C}(38)$ & $1.392(3)$ \\
\hline$C(37)-C(42)$ & $1.388(3)$ \\
\hline $\mathrm{C}(38)-\mathrm{H}(38)$ & 0.9500 \\
\hline $\mathrm{C}(38)-\mathrm{C}(39)$ & $1.396(3)$ \\
\hline C(39)-H(39) & 0.9500 \\
\hline$C(39)-C(40)$ & $1.376(4)$ \\
\hline $\mathrm{C}(40)-\mathrm{H}(40)$ & 0.9500 \\
\hline $\mathrm{C}(40)-\mathrm{C}(41)$ & $1.381(4)$ \\
\hline $\mathrm{C}(41)-\mathrm{H}(41)$ & 0.9500 \\
\hline $\mathrm{C}(41)-\mathrm{C}(42)$ & $1.393(4)$ \\
\hline $\mathrm{C}(42)-\mathrm{H}(42)$ & 0.9500 \\
\hline $\mathrm{C}(43)-\mathrm{H}(43 \mathrm{~A})$ & 0.9900 \\
\hline $\mathrm{C}(43)-\mathrm{H}(43 \mathrm{~B})$ & 0.9900 \\
\hline $\mathrm{C}(43)-\mathrm{H}(43 \mathrm{C})$ & 0.9900 \\
\hline $\mathrm{C}(43)-\mathrm{H}(43 \mathrm{D})$ & 0.9900 \\
\hline $\mathrm{C}(43)-\mathrm{Cl}(1)$ & $1.762(5)$ \\
\hline $\mathrm{C}(43)-\mathrm{Cl}(2)$ & $1.779(4)$ \\
\hline $\mathrm{C}(43)-\mathrm{Cl}(1 \mathrm{~A})$ & $1.762(12)$ \\
\hline $\mathrm{C}(43)-\mathrm{Cl}(2 \mathrm{~A})$ & $1.746(8)$ \\
\hline $\mathrm{P}(1)-\mathrm{Pd}(1)-\mathrm{I}(1)$ & $91.800(16)$ \\
\hline $\mathrm{P}(1)-\mathrm{Pd}(1)-\mathrm{P}(2)$ & $174.10(2)$ \\
\hline
\end{tabular}




\begin{tabular}{|c|c|}
\hline $\mathrm{P}(2)-\mathrm{Pd}(1)-\mathrm{I}(1)$ & $92.824(16)$ \\
\hline $\mathrm{C}(37)-\mathrm{Pd}(1)-\mathrm{I}(1)$ & $170.22(7)$ \\
\hline $\mathrm{C}(37)-\mathrm{Pd}(1)-\mathrm{P}(1)$ & $86.52(6)$ \\
\hline $\mathrm{C}(37)-\mathrm{Pd}(1)-\mathrm{P}(2)$ & $89.53(6)$ \\
\hline $\mathrm{C}(1)-\mathrm{P}(1)-\mathrm{Pd}(1)$ & $113.08(8)$ \\
\hline $\mathrm{C}(7)-\mathrm{P}(1)-\mathrm{Pd}(1)$ & $111.68(8)$ \\
\hline $\mathrm{C}(7)-\mathrm{P}(1)-\mathrm{C}(1)$ & $107.93(11)$ \\
\hline $\mathrm{C}(7)-\mathrm{P}(1)-\mathrm{C}(13)$ & $102.43(11)$ \\
\hline $\mathrm{C}(13)-\mathrm{P}(1)-\mathrm{Pd}(1)$ & $118.30(7)$ \\
\hline $\mathrm{C}(13)-\mathrm{P}(1)-\mathrm{C}(1)$ & $102.33(10)$ \\
\hline$C(19)-P(2)-P d(1)$ & $118.77(7)$ \\
\hline $\mathrm{C}(25)-\mathrm{P}(2)-\mathrm{Pd}(1)$ & $114.45(8)$ \\
\hline $\mathrm{C}(25)-\mathrm{P}(2)-\mathrm{C}(19)$ & $103.56(10)$ \\
\hline $\mathrm{C}(25)-\mathrm{P}(2)-\mathrm{C}(31)$ & $104.70(10)$ \\
\hline $\mathrm{C}(31)-\mathrm{P}(2)-\mathrm{Pd}(1)$ & $110.20(7)$ \\
\hline $\mathrm{C}(31)-\mathrm{P}(2)-\mathrm{C}(19)$ & $103.75(10)$ \\
\hline $\mathrm{C}(2)-\mathrm{C}(1)-\mathrm{P}(1)$ & $123.50(18)$ \\
\hline$C(2)-C(1)-C(6)$ & $119.0(2)$ \\
\hline $\mathrm{C}(6)-\mathrm{C}(1)-\mathrm{P}(1)$ & $117.47(17)$ \\
\hline $\mathrm{C}(1)-\mathrm{C}(2)-\mathrm{H}(2)$ & 119.7 \\
\hline$C(3)-C(2)-C(1)$ & $120.6(2)$ \\
\hline $\mathrm{C}(3)-\mathrm{C}(2)-\mathrm{H}(2)$ & 119.7 \\
\hline $\mathrm{C}(2)-\mathrm{C}(3)-\mathrm{H}(3)$ & 120.8 \\
\hline$C(4)-C(3)-C(2)$ & $118.3(2)$ \\
\hline $\mathrm{C}(4)-\mathrm{C}(3)-\mathrm{H}(3)$ & 120.8 \\
\hline
\end{tabular}




\begin{tabular}{|c|c|}
\hline $\mathrm{F}(1)-\mathrm{C}(4)-\mathrm{C}(3)$ & $118.5(2)$ \\
\hline $\mathrm{F}(1)-\mathrm{C}(4)-\mathrm{C}(5)$ & 118.2(2) \\
\hline$C(3)-C(4)-C(5)$ & $123.3(2)$ \\
\hline $\mathrm{C}(4)-\mathrm{C}(5)-\mathrm{H}(5)$ & 121.1 \\
\hline$C(4)-C(5)-C(6)$ & $117.7(2)$ \\
\hline $\mathrm{C}(6)-\mathrm{C}(5)-\mathrm{H}(5)$ & 121.1 \\
\hline $\mathrm{C}(1)-\mathrm{C}(6)-\mathrm{H}(6)$ & 119.5 \\
\hline$C(5)-C(6)-C(1)$ & $121.0(2)$ \\
\hline $\mathrm{C}(5)-\mathrm{C}(6)-\mathrm{H}(6)$ & 119.5 \\
\hline $\mathrm{C}(8)-\mathrm{C}(7)-\mathrm{P}(1)$ & $119.52(18)$ \\
\hline $\mathrm{C}(8)-\mathrm{C}(7)-\mathrm{C}(12)$ & $118.7(2)$ \\
\hline $\mathrm{C}(12)-\mathrm{C}(7)-\mathrm{P}(1)$ & $121.63(18)$ \\
\hline $\mathrm{C}(7)-\mathrm{C}(8)-\mathrm{H}(8)$ & 119.6 \\
\hline $\mathrm{C}(9)-\mathrm{C}(8)-\mathrm{C}(7)$ & $120.8(2)$ \\
\hline $\mathrm{C}(9)-\mathrm{C}(8)-\mathrm{H}(8)$ & 119.6 \\
\hline $\mathrm{C}(8)-\mathrm{C}(9)-\mathrm{H}(9)$ & 121.0 \\
\hline $\mathrm{C}(10)-\mathrm{C}(9)-\mathrm{C}(8)$ & $118.1(2)$ \\
\hline $\mathrm{C}(10)-\mathrm{C}(9)-\mathrm{H}(9)$ & 121.0 \\
\hline $\mathrm{F}(2)-\mathrm{C}(10)-\mathrm{C}(9)$ & $118.2(2)$ \\
\hline $\mathrm{F}(2)-\mathrm{C}(10)-\mathrm{C}(11)$ & $118.9(2)$ \\
\hline$C(11)-C(10)-C(9)$ & $122.9(2)$ \\
\hline $\mathrm{C}(10)-\mathrm{C}(11)-\mathrm{H}(11)$ & 120.8 \\
\hline$C(10)-C(11)-C(12)$ & $118.4(2)$ \\
\hline $\mathrm{C}(12)-\mathrm{C}(11)-\mathrm{H}(11)$ & 120.8 \\
\hline $\mathrm{C}(7)-\mathrm{C}(12)-\mathrm{H}(12)$ & 119.5 \\
\hline
\end{tabular}




\begin{tabular}{|c|c|}
\hline$C(11)-C(12)-C(7)$ & $121.1(2)$ \\
\hline $\mathrm{C}(11)-\mathrm{C}(12)-\mathrm{H}(12)$ & 119.5 \\
\hline $\mathrm{C}(14)-\mathrm{C}(13)-\mathrm{P}(1)$ & $121.67(18)$ \\
\hline $\mathrm{C}(18)-\mathrm{C}(13)-\mathrm{P}(1)$ & $120.05(18)$ \\
\hline $\mathrm{C}(18)-\mathrm{C}(13)-\mathrm{C}(14)$ & $118.1(2)$ \\
\hline $\mathrm{C}(13)-\mathrm{C}(14)-\mathrm{H}(14)$ & 119.6 \\
\hline$C(15)-C(14)-C(13)$ & $120.8(2)$ \\
\hline $\mathrm{C}(15)-\mathrm{C}(14)-\mathrm{H}(14)$ & 119.6 \\
\hline $\mathrm{C}(14)-\mathrm{C}(15)-\mathrm{H}(15)$ & 120.5 \\
\hline$C(16)-C(15)-C(14)$ & $118.9(2)$ \\
\hline $\mathrm{C}(16)-\mathrm{C}(15)-\mathrm{H}(15)$ & 120.5 \\
\hline $\mathrm{F}(3)-\mathrm{C}(16)-\mathrm{C}(15)$ & $119.4(2)$ \\
\hline $\mathrm{F}(3)-\mathrm{C}(16)-\mathrm{C}(17)$ & $118.4(2)$ \\
\hline$C(17)-C(16)-C(15)$ & $122.3(2)$ \\
\hline $\mathrm{C}(16)-\mathrm{C}(17)-\mathrm{H}(17)$ & 120.8 \\
\hline$C(16)-C(17)-C(18)$ & $118.3(2)$ \\
\hline $\mathrm{C}(18)-\mathrm{C}(17)-\mathrm{H}(17)$ & 120.8 \\
\hline $\mathrm{C}(13)-\mathrm{C}(18)-\mathrm{H}(18)$ & 119.2 \\
\hline $\mathrm{C}(17)-\mathrm{C}(18)-\mathrm{C}(13)$ & $121.5(2)$ \\
\hline $\mathrm{C}(17)-\mathrm{C}(18)-\mathrm{H}(18)$ & 119.2 \\
\hline $\mathrm{C}(20)-\mathrm{C}(19)-\mathrm{P}(2)$ & $118.56(17)$ \\
\hline $\mathrm{C}(24)-\mathrm{C}(19)-\mathrm{P}(2)$ & $122.63(18)$ \\
\hline$C(24)-C(19)-C(20)$ & $118.7(2)$ \\
\hline$C(19)-C(20)-H(20)$ & 119.6 \\
\hline$C(21)-C(20)-C(19)$ & $120.7(2)$ \\
\hline
\end{tabular}




\begin{tabular}{|c|c|}
\hline $\mathrm{C}(21)-\mathrm{C}(20)-\mathrm{H}(20)$ & 119.6 \\
\hline $\mathrm{C}(20)-\mathrm{C}(21)-\mathrm{H}(21)$ & 121.0 \\
\hline$C(22)-C(21)-C(20)$ & $118.1(2)$ \\
\hline $\mathrm{C}(22)-\mathrm{C}(21)-\mathrm{H}(21)$ & 121.0 \\
\hline $\mathrm{F}(4)-\mathrm{C}(22)-\mathrm{C}(21)$ & $118.3(2)$ \\
\hline $\mathrm{F}(4)-\mathrm{C}(22)-\mathrm{C}(23)$ & $118.6(2)$ \\
\hline$C(23)-C(22)-C(21)$ & $123.1(2)$ \\
\hline $\mathrm{C}(22)-\mathrm{C}(23)-\mathrm{H}(23)$ & 120.8 \\
\hline$C(22)-C(23)-C(24)$ & $118.4(2)$ \\
\hline $\mathrm{C}(24)-\mathrm{C}(23)-\mathrm{H}(23)$ & 120.8 \\
\hline $\mathrm{C}(19)-\mathrm{C}(24)-\mathrm{H}(24)$ & 119.5 \\
\hline$C(23)-C(24)-C(19)$ & $120.9(2)$ \\
\hline $\mathrm{C}(23)-\mathrm{C}(24)-\mathrm{H}(24)$ & 119.5 \\
\hline$C(26)-C(25)-P(2)$ & $120.83(18)$ \\
\hline $\mathrm{C}(30)-\mathrm{C}(25)-\mathrm{P}(2)$ & $119.40(17)$ \\
\hline$C(30)-C(25)-C(26)$ & $119.6(2)$ \\
\hline $\mathrm{C}(25)-\mathrm{C}(26)-\mathrm{H}(26)$ & 119.9 \\
\hline$C(27)-C(26)-C(25)$ & $120.3(2)$ \\
\hline $\mathrm{C}(27)-\mathrm{C}(26)-\mathrm{H}(26)$ & 119.9 \\
\hline $\mathrm{C}(26)-\mathrm{C}(27)-\mathrm{H}(27)$ & 120.8 \\
\hline$C(28)-C(27)-C(26)$ & $118.3(2)$ \\
\hline $\mathrm{C}(28)-\mathrm{C}(27)-\mathrm{H}(27)$ & 120.8 \\
\hline $\mathrm{F}(5)-\mathrm{C}(28)-\mathrm{C}(27)$ & $118.7(2)$ \\
\hline $\mathrm{F}(5)-\mathrm{C}(28)-\mathrm{C}(29)$ & $118.4(2)$ \\
\hline $\mathrm{C}(29)-\mathrm{C}(28)-\mathrm{C}(27)$ & $123.0(2)$ \\
\hline
\end{tabular}




\begin{tabular}{|c|c|}
\hline $\mathrm{C}(28)-\mathrm{C}(29)-\mathrm{H}(29)$ & 120.9 \\
\hline $\mathrm{C}(28)-\mathrm{C}(29)-\mathrm{C}(30)$ & $118.3(2)$ \\
\hline C(30)-C(29)-H(29) & 120.9 \\
\hline $\mathrm{C}(25)-\mathrm{C}(30)-\mathrm{C}(29)$ & $120.6(2)$ \\
\hline $\mathrm{C}(25)-\mathrm{C}(30)-\mathrm{H}(30)$ & 119.7 \\
\hline $\mathrm{C}(29)-\mathrm{C}(30)-\mathrm{H}(30)$ & 119.7 \\
\hline $\mathrm{C}(32)-\mathrm{C}(31)-\mathrm{P}(2)$ & $118.83(17)$ \\
\hline$C(36)-C(31)-P(2)$ & $122.16(18)$ \\
\hline $\mathrm{C}(36)-\mathrm{C}(31)-\mathrm{C}(32)$ & $118.9(2)$ \\
\hline $\mathrm{C}(31)-\mathrm{C}(32)-\mathrm{H}(32)$ & 119.5 \\
\hline$C(33)-C(32)-C(31)$ & $121.0(2)$ \\
\hline $\mathrm{C}(33)-\mathrm{C}(32)-\mathrm{H}(32)$ & 119.5 \\
\hline $\mathrm{C}(32)-\mathrm{C}(33)-\mathrm{H}(33)$ & 121.0 \\
\hline $\mathrm{C}(34)-\mathrm{C}(33)-\mathrm{C}(32)$ & $118.0(2)$ \\
\hline $\mathrm{C}(34)-\mathrm{C}(33)-\mathrm{H}(33)$ & 121.0 \\
\hline $\mathrm{F}(6)-\mathrm{C}(34)-\mathrm{C}(33)$ & $118.1(2)$ \\
\hline $\mathrm{F}(6)-\mathrm{C}(34)-\mathrm{C}(35)$ & 118.7(2) \\
\hline$C(35)-C(34)-C(33)$ & $123.2(2)$ \\
\hline $\mathrm{C}(34)-\mathrm{C}(35)-\mathrm{H}(35)$ & 121.0 \\
\hline$C(34)-C(35)-C(36)$ & $118.0(2)$ \\
\hline $\mathrm{C}(36)-\mathrm{C}(35)-\mathrm{H}(35)$ & 121.0 \\
\hline $\mathrm{C}(31)-\mathrm{C}(36)-\mathrm{C}(35)$ & $120.8(2)$ \\
\hline $\mathrm{C}(31)-\mathrm{C}(36)-\mathrm{H}(36)$ & 119.6 \\
\hline $\mathrm{C}(35)-\mathrm{C}(36)-\mathrm{H}(36)$ & 119.6 \\
\hline $\mathrm{C}(38)-\mathrm{C}(37)-\mathrm{Pd}(1)$ & $118.05(18)$ \\
\hline
\end{tabular}




\begin{tabular}{|c|c|}
\hline $\mathrm{C}(42)-\mathrm{C}(37)-\mathrm{Pd}(1)$ & $122.73(18)$ \\
\hline $\mathrm{C}(42)-\mathrm{C}(37)-\mathrm{C}(38)$ & $119.2(2)$ \\
\hline $\mathrm{C}(37)-\mathrm{C}(38)-\mathrm{H}(38)$ & 119.9 \\
\hline$C(37)-C(38)-C(39)$ & $120.1(2)$ \\
\hline $\mathrm{C}(39)-\mathrm{C}(38)-\mathrm{H}(38)$ & 119.9 \\
\hline $\mathrm{C}(38)-\mathrm{C}(39)-\mathrm{H}(39)$ & 119.8 \\
\hline $\mathrm{C}(40)-\mathrm{C}(39)-\mathrm{C}(38)$ & $120.4(3)$ \\
\hline C(40)-C(39)-H(39) & 119.8 \\
\hline $\mathrm{C}(39)-\mathrm{C}(40)-\mathrm{H}(40)$ & 120.2 \\
\hline$C(39)-C(40)-C(41)$ & $119.6(2)$ \\
\hline $\mathrm{C}(41)-\mathrm{C}(40)-\mathrm{H}(40)$ & 120.2 \\
\hline $\mathrm{C}(40)-\mathrm{C}(41)-\mathrm{H}(41)$ & 119.7 \\
\hline$C(40)-C(41)-C(42)$ & $120.7(3)$ \\
\hline $\mathrm{C}(42)-\mathrm{C}(41)-\mathrm{H}(41)$ & 119.7 \\
\hline$C(37)-C(42)-C(41)$ & $120.0(2)$ \\
\hline $\mathrm{C}(37)-\mathrm{C}(42)-\mathrm{H}(42)$ & 120.0 \\
\hline $\mathrm{C}(41)-\mathrm{C}(42)-\mathrm{H}(42)$ & 120.0 \\
\hline $\mathrm{H}(43 \mathrm{~A})-\mathrm{C}(43)-\mathrm{H}(43 \mathrm{~B})$ & 107.9 \\
\hline $\mathrm{H}(43 \mathrm{C})-\mathrm{C}(43)-\mathrm{H}(43 \mathrm{D})$ & 108.8 \\
\hline $\mathrm{Cl}(1)-\mathrm{C}(43)-\mathrm{H}(43 \mathrm{~A})$ & 109.2 \\
\hline $\mathrm{Cl}(1)-\mathrm{C}(43)-\mathrm{H}(43 \mathrm{~B})$ & 109.2 \\
\hline $\mathrm{Cl}(1)-\mathrm{C}(43)-\mathrm{Cl}(2)$ & 112.1(3) \\
\hline $\mathrm{Cl}(2)-\mathrm{C}(43)-\mathrm{H}(43 \mathrm{~A})$ & 109.2 \\
\hline $\mathrm{Cl}(2)-\mathrm{C}(43)-\mathrm{H}(43 \mathrm{~B})$ & 109.2 \\
\hline $\mathrm{Cl}(1 \mathrm{~A})-\mathrm{C}(43)-\mathrm{H}(43 \mathrm{C})$ & 110.6 \\
\hline
\end{tabular}




$\begin{array}{lc}\mathrm{Cl}(1 \mathrm{~A})-\mathrm{C}(43)-\mathrm{H}(43 \mathrm{D}) & 110.6 \\ \mathrm{Cl}(2 \mathrm{~A})-\mathrm{C}(43)-\mathrm{H}(43 \mathrm{C}) & 110.6 \\ \mathrm{Cl}(2 \mathrm{~A})-\mathrm{C}(43)-\mathrm{H}(43 \mathrm{D}) & 110.6 \\ \mathrm{Cl}(2 \mathrm{~A})-\mathrm{C}(43)-\mathrm{Cl}(1 \mathrm{~A}) & 105.5(6)\end{array}$


Table S5. Anisotropic displacement parameters $\left(\AA^{2} \times 10^{3}\right)$ for Engle213. The anisotropic displacement factor exponent takes the form: $-2 \pi^{2}\left[h^{2} a^{* 2} U^{11}+\ldots+2 h k a^{*} b^{*} U^{12}\right]$

\begin{tabular}{|c|c|c|c|c|c|c|}
\hline & $\mathrm{U}^{11}$ & $\mathrm{U}^{22}$ & $\mathrm{U}^{33}$ & $\mathrm{U}^{23}$ & $\mathrm{U}^{13}$ & $\mathrm{U}^{12}$ \\
\hline $\mathrm{I}(1)$ & $15(1)$ & $16(1)$ & $14(1)$ & $0(1)$ & $4(1)$ & $3(1)$ \\
\hline $\operatorname{Pd}(1)$ & 11(1) & $11(1)$ & $9(1)$ & $0(1)$ & $3(1)$ & $0(1)$ \\
\hline $\mathrm{P}(1)$ & $12(1)$ & $12(1)$ & $9(1)$ & $0(1)$ & $3(1)$ & $0(1)$ \\
\hline $\mathrm{P}(2)$ & $12(1)$ & $12(1)$ & $9(1)$ & 1(1) & $2(1)$ & $-1(1)$ \\
\hline $\mathrm{F}(1)$ & $13(1)$ & $36(1)$ & $24(1)$ & $1(1)$ & $1(1)$ & $-1(1)$ \\
\hline $\mathrm{F}(2)$ & $51(1)$ & $17(1)$ & $56(1)$ & $12(1)$ & $31(1)$ & $-3(1)$ \\
\hline $\mathrm{F}(3)$ & $46(1)$ & $17(1)$ & $28(1)$ & $-4(1)$ & $22(1)$ & $7(1)$ \\
\hline $\mathrm{F}(4)$ & $24(1)$ & $20(1)$ & $31(1)$ & $2(1)$ & $6(1)$ & $11(1)$ \\
\hline $\mathrm{F}(5)$ & $30(1)$ & 19(1) & $21(1)$ & $-7(1)$ & $2(1)$ & $-8(1)$ \\
\hline $\mathrm{F}(6)$ & $22(1)$ & $47(1)$ & $31(1)$ & $-7(1)$ & $17(1)$ & $-11(1)$ \\
\hline $\mathrm{C}(1)$ & $14(1)$ & $15(1)$ & $10(1)$ & $-4(1)$ & $3(1)$ & $0(1)$ \\
\hline$C(2)$ & $19(1)$ & $13(1)$ & $13(1)$ & $0(1)$ & $5(1)$ & $-2(1)$ \\
\hline$C(3)$ & $20(1)$ & $18(1)$ & $14(1)$ & $1(1)$ & $0(1)$ & $6(1)$ \\
\hline$C(4)$ & $12(1)$ & $24(1)$ & $16(1)$ & $-6(1)$ & $2(1)$ & $0(1)$ \\
\hline$C(5)$ & $19(1)$ & $21(1)$ & $16(1)$ & $2(1)$ & $6(1)$ & $-3(1)$ \\
\hline$C(6)$ & $17(1)$ & $19(1)$ & $13(1)$ & $2(1)$ & $5(1)$ & $1(1)$ \\
\hline$C(7)$ & $17(1)$ & $12(1)$ & $16(1)$ & $2(1)$ & $7(1)$ & $2(1)$ \\
\hline$C(8)$ & $17(1)$ & $16(1)$ & $19(1)$ & $0(1)$ & $8(1)$ & $1(1)$ \\
\hline$C(9)$ & $22(1)$ & $14(1)$ & $32(2)$ & $-2(1)$ & $11(1)$ & $0(1)$ \\
\hline$C(10)$ & $23(1)$ & $14(1)$ & $40(2)$ & $10(1)$ & $19(1)$ & $4(1)$ \\
\hline
\end{tabular}




\begin{tabular}{|c|c|c|c|c|c|c|}
\hline $\mathrm{C}(11)$ & $26(1)$ & $25(1)$ & $22(1)$ & 11(1) & $13(1)$ & $8(1)$ \\
\hline$C(12)$ & $22(1)$ & $17(1)$ & $16(1)$ & 1(1) & $6(1)$ & $4(1)$ \\
\hline$C(13)$ & $15(1)$ & $14(1)$ & $9(1)$ & $2(1)$ & 1(1) & $2(1)$ \\
\hline $\mathrm{C}(14)$ & $19(1)$ & $15(1)$ & $14(1)$ & $3(1)$ & $5(1)$ & $-2(1)$ \\
\hline$C(15)$ & $30(2)$ & $12(1)$ & $17(1)$ & $-1(1)$ & $5(1)$ & $0(1)$ \\
\hline$C(16)$ & $31(2)$ & $15(1)$ & $14(1)$ & $2(1)$ & $9(1)$ & $7(1)$ \\
\hline$C(17)$ & $22(1)$ & $22(1)$ & $18(1)$ & $2(1)$ & $9(1)$ & $3(1)$ \\
\hline $\mathrm{C}(18)$ & $17(1)$ & $17(1)$ & $15(1)$ & $-2(1)$ & $2(1)$ & $-1(1)$ \\
\hline C(19) & $14(1)$ & $13(1)$ & $8(1)$ & $0(1)$ & $2(1)$ & $0(1)$ \\
\hline$C(20)$ & $18(1)$ & $21(1)$ & $12(1)$ & $4(1)$ & $4(1)$ & $-1(1)$ \\
\hline $\mathrm{C}(21)$ & $12(1)$ & $27(1)$ & $15(1)$ & $2(1)$ & $3(1)$ & $2(1)$ \\
\hline$C(22)$ & $20(1)$ & $15(1)$ & $14(1)$ & $-1(1)$ & 1(1) & $6(1)$ \\
\hline$C(23)$ & $22(1)$ & $13(1)$ & $20(1)$ & 1(1) & $5(1)$ & $-1(1)$ \\
\hline$C(24)$ & $15(1)$ & $18(1)$ & $17(1)$ & $-1(1)$ & $6(1)$ & $-1(1)$ \\
\hline$C(25)$ & $10(1)$ & $16(1)$ & 11(1) & $-2(1)$ & $4(1)$ & $-1(1)$ \\
\hline$C(26)$ & $19(1)$ & $14(1)$ & $15(1)$ & $4(1)$ & $4(1)$ & $1(1)$ \\
\hline$C(27)$ & $18(1)$ & $24(1)$ & 11(1) & $0(1)$ & $-1(1)$ & $0(1)$ \\
\hline$C(28)$ & $16(1)$ & $19(1)$ & $18(1)$ & $-6(1)$ & $7(1)$ & $-5(1)$ \\
\hline$C(29)$ & $20(1)$ & $12(1)$ & $20(1)$ & $2(1)$ & $4(1)$ & $-2(1)$ \\
\hline$C(30)$ & $16(1)$ & $19(1)$ & 11(1) & 1(1) & $3(1)$ & $-1(1)$ \\
\hline $\mathrm{C}(31)$ & $13(1)$ & $14(1)$ & $11(1)$ & $3(1)$ & $2(1)$ & $3(1)$ \\
\hline$C(32)$ & $17(1)$ & $13(1)$ & 11(1) & $0(1)$ & $3(1)$ & $0(1)$ \\
\hline$C(33)$ & $14(1)$ & $18(1)$ & $17(1)$ & $2(1)$ & $2(1)$ & $-3(1)$ \\
\hline$C(34)$ & $14(1)$ & $26(1)$ & $19(1)$ & $4(1)$ & $7(1)$ & $-1(1)$ \\
\hline$C(35)$ & $20(1)$ & $25(1)$ & $17(1)$ & $-6(1)$ & $9(1)$ & $-1(1)$ \\
\hline
\end{tabular}




\begin{tabular}{lllllll}
$\mathrm{C}(36)$ & $16(1)$ & $18(1)$ & $15(1)$ & $-2(1)$ & $1(1)$ & $-3(1)$ \\
$\mathrm{C}(37)$ & $15(1)$ & $20(1)$ & $9(1)$ & $1(1)$ & $3(1)$ & $3(1)$ \\
$\mathrm{C}(38)$ & $16(1)$ & $24(1)$ & $16(1)$ & $2(1)$ & $4(1)$ & $1(1)$ \\
$\mathrm{C}(39)$ & $16(1)$ & $44(2)$ & $22(1)$ & $7(1)$ & $8(1)$ & $4(1)$ \\
$\mathrm{C}(40)$ & $28(2)$ & $50(2)$ & $26(2)$ & $9(1)$ & $12(1)$ & $23(1)$ \\
$\mathrm{C}(41)$ & $38(2)$ & $26(2)$ & $21(1)$ & $-1(1)$ & $6(1)$ & $18(1)$ \\
$\mathrm{C}(42)$ & $23(1)$ & $20(1)$ & $14(1)$ & $0(1)$ & $3(1)$ & $4(1)$ \\
$\mathrm{C}(43)$ & $19(1)$ & $27(2)$ & $31(2)$ & $9(1)$ & $5(1)$ & $-2(1)$ \\
$\mathrm{Cl}(1)$ & $32(1)$ & $22(1)$ & $24(1)$ & $3(1)$ & $1(1)$ & $-5(1)$ \\
$\mathrm{Cl}(2)$ & $27(1)$ & $49(2)$ & $28(1)$ & $9(1)$ & $-4(1)$ & $-4(1)$ \\
$\mathrm{Cl}(1 \mathrm{~A})$ & $32(1)$ & $22(1)$ & $24(1)$ & $3(1)$ & $1(1)$ & $-5(1)$ \\
$\mathrm{Cl}(2 \mathrm{~A})$ & $27(1)$ & $49(2)$ & $28(1)$ & $9(1)$ & $-4(1)$ & $-4(1)$ \\
& & & & & \\
\hline
\end{tabular}


Table S6. Hydrogen coordinates ( x 104) and isotropic displacement parameters $\left(\AA^{2} \times 10^{3}\right)$ for Engle213.

\begin{tabular}{|c|c|c|c|c|}
\hline & $\mathrm{x}$ & $\mathrm{y}$ & $\mathrm{z}$ & $\mathrm{U}(\mathrm{eq})$ \\
\hline $\mathrm{H}(2)$ & 4727 & 3788 & 4416 & 18 \\
\hline $\mathrm{H}(3)$ & 6323 & 4076 & 4741 & 22 \\
\hline $\mathrm{H}(5)$ & 6192 & 6680 & 3592 & 22 \\
\hline $\mathrm{H}(6)$ & 4611 & 6329 & 3238 & 19 \\
\hline $\mathrm{H}(8)$ & 2535 & 2755 & 2996 & 20 \\
\hline $\mathrm{H}(9)$ & 1991 & 1009 & 3298 & 26 \\
\hline $\mathrm{H}(11)$ & 2630 & 2199 & 4925 & 28 \\
\hline $\mathrm{H}(12)$ & 3176 & 3931 & 4625 & 22 \\
\hline $\mathrm{H}(14)$ & 3884 & 7136 & 4061 & 19 \\
\hline $\mathrm{H}(15)$ & 3259 & 8526 & 4562 & 24 \\
\hline $\mathrm{H}(17)$ & 1034 & 6470 & 4467 & 24 \\
\hline $\mathrm{H}(18)$ & 1641 & 5098 & 3948 & 20 \\
\hline $\mathrm{H}(20)$ & 423 & 6000 & 1566 & 20 \\
\hline $\mathrm{H}(21)$ & -521 & 7656 & 1340 & 22 \\
\hline $\mathrm{H}(23)$ & 1480 & 9292 & 782 & 22 \\
\hline $\mathrm{H}(24)$ & 2433 & 7655 & 1022 & 20 \\
\hline $\mathrm{H}(26)$ & 1074 & 5126 & 432 & 19 \\
\hline $\mathrm{H}(27)$ & 380 & 3524 & -112 & 22 \\
\hline H(29) & 1337 & 1392 & 1241 & 21 \\
\hline
\end{tabular}




\begin{tabular}{lcccc}
$\mathrm{H}(30)$ & 2048 & 2989 & 1781 & 18 \\
$\mathrm{H}(32)$ & 3858 & 6865 & 1841 & 17 \\
$\mathrm{H}(33)$ & 5112 & 7240 & 1454 & 20 \\
$\mathrm{H}(35)$ & 4215 & 4744 & 260 & 24 \\
$\mathrm{H}(36)$ & 2918 & 4438 & 623 & 20 \\
$\mathrm{H}(38)$ & 910 & 4384 & 2634 & 22 \\
$\mathrm{H}(39)$ & -420 & 5274 & 2778 & 33 \\
$\mathrm{H}(40)$ & -464 & 7282 & 2906 & 40 \\
$\mathrm{H}(41)$ & 826 & 8407 & 2895 & 34 \\
$\mathrm{H}(42)$ & 2151 & 7539 & 2737 & 23 \\
$\mathrm{H}(43 \mathrm{~A})$ & 4103 & 9920 & 2501 & 31 \\
$\mathrm{H}(43 \mathrm{~B})$ & 3786 & 10983 & 2837 & 31 \\
$\mathrm{H}(43 \mathrm{C})$ & 3574 & 10964 & 2856 & 31 \\
$\mathrm{H}(43 \mathrm{D})$ & 4203 & 10149 & 2564 & 31 \\
\hline & & & \\
\hline
\end{tabular}




\section{NMR SPECTRA}

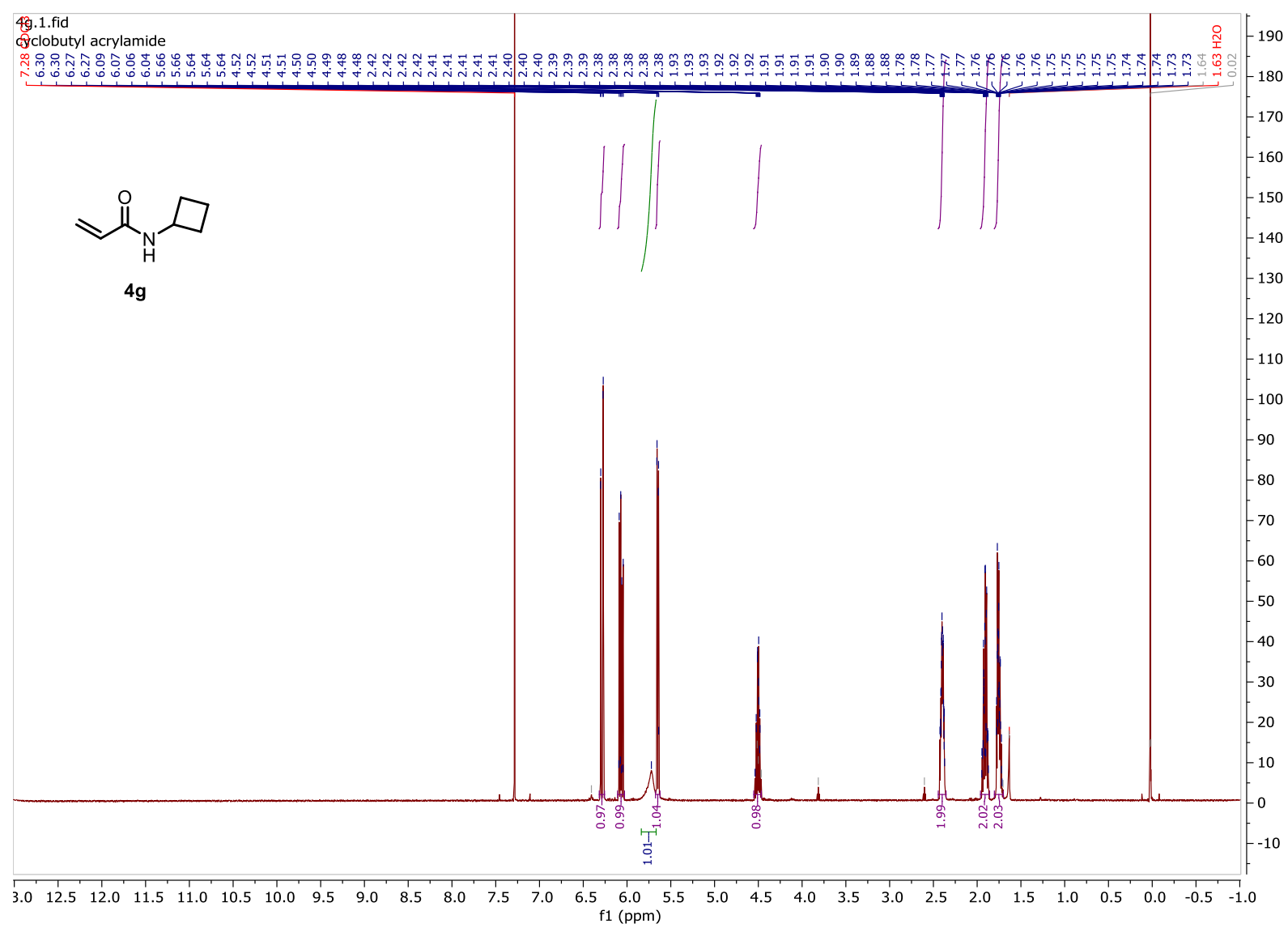




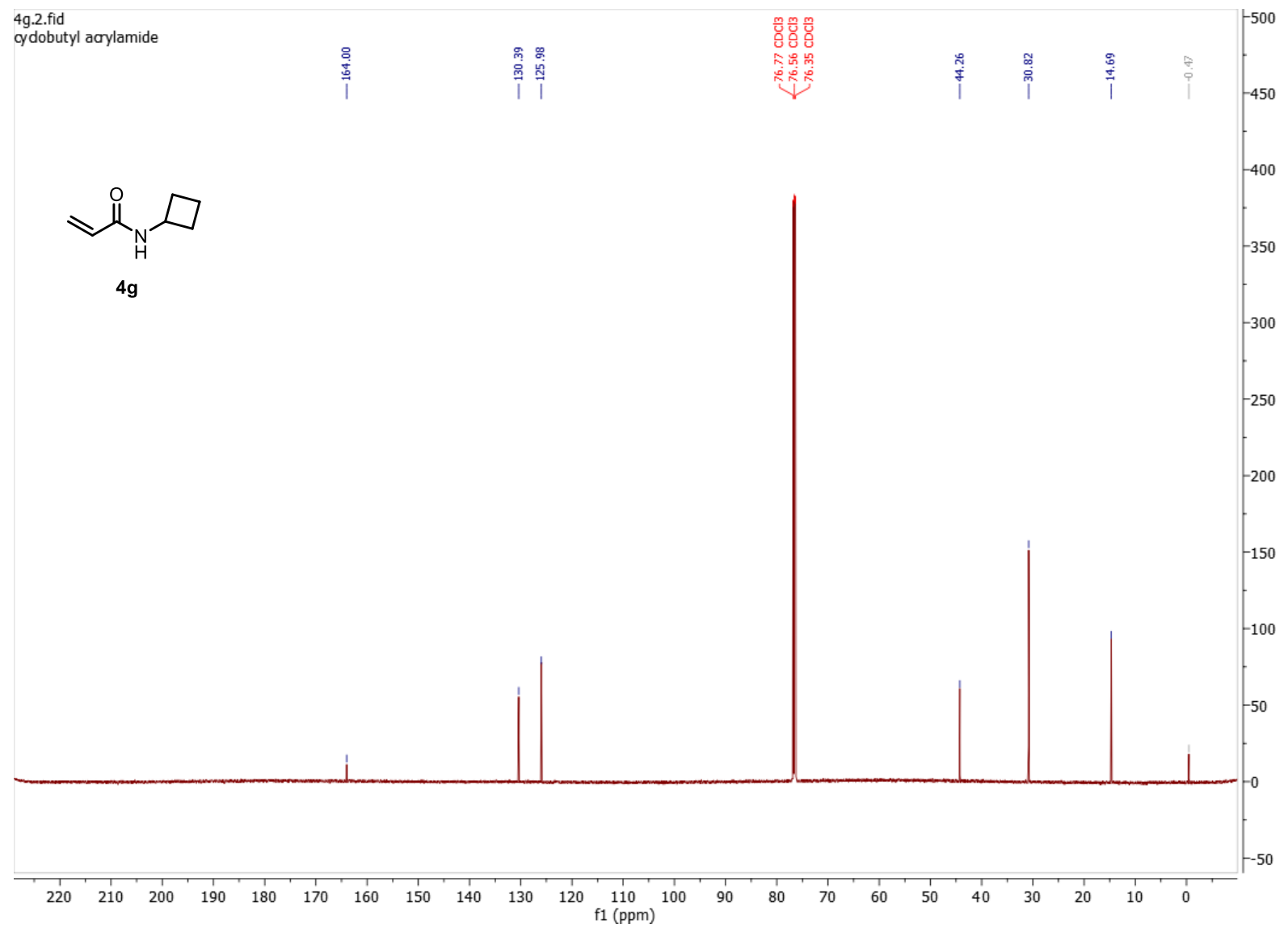




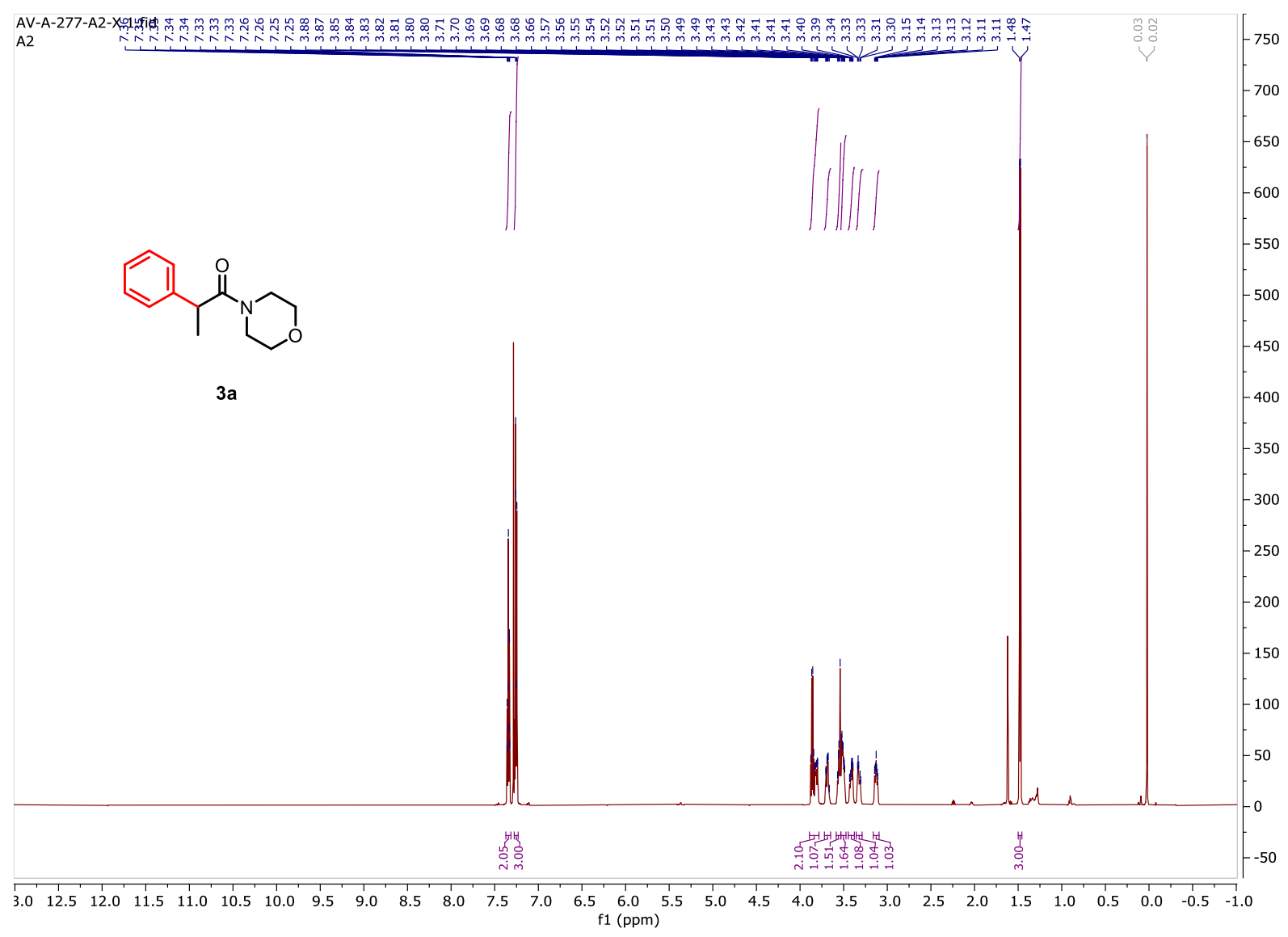




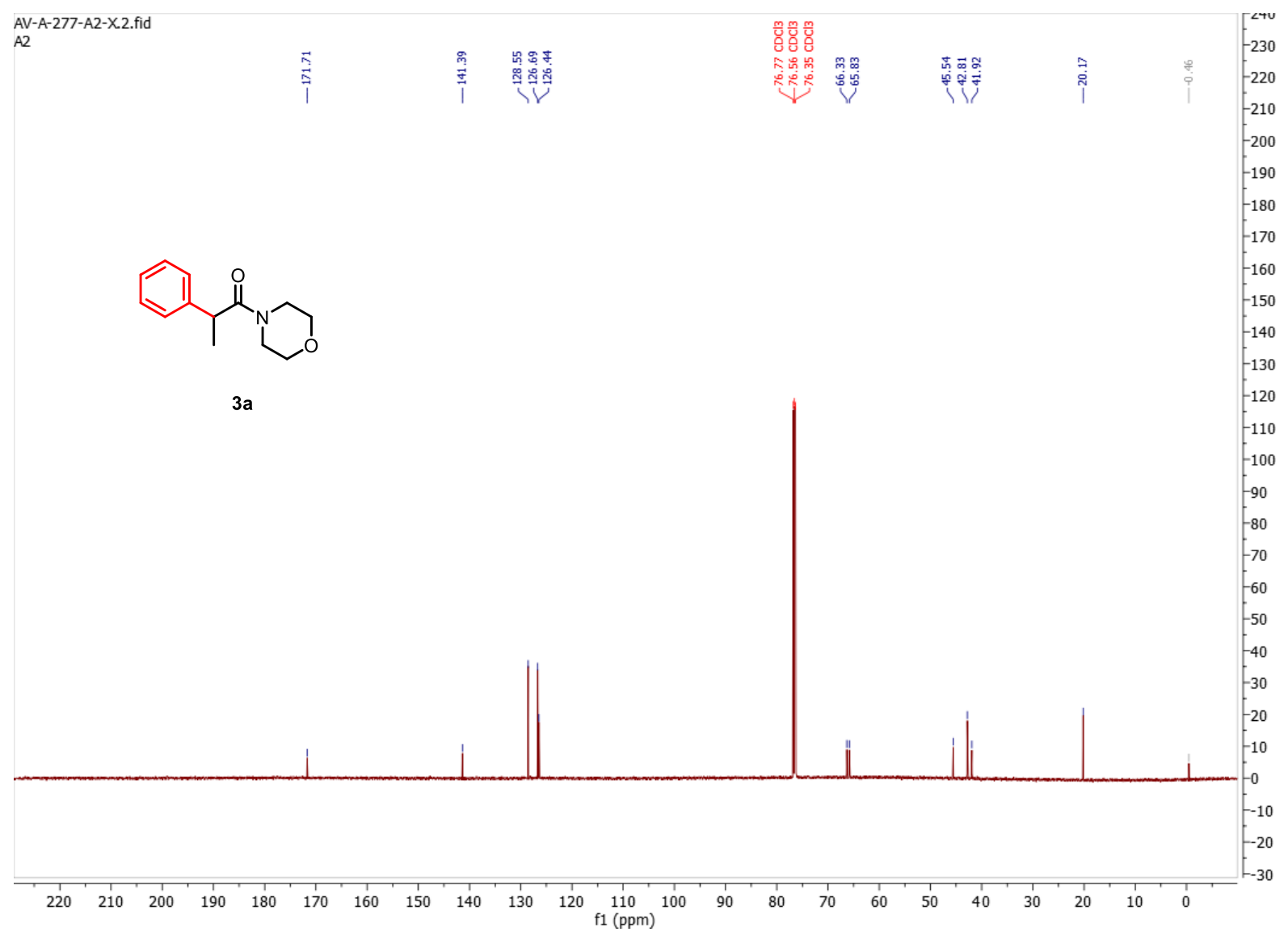




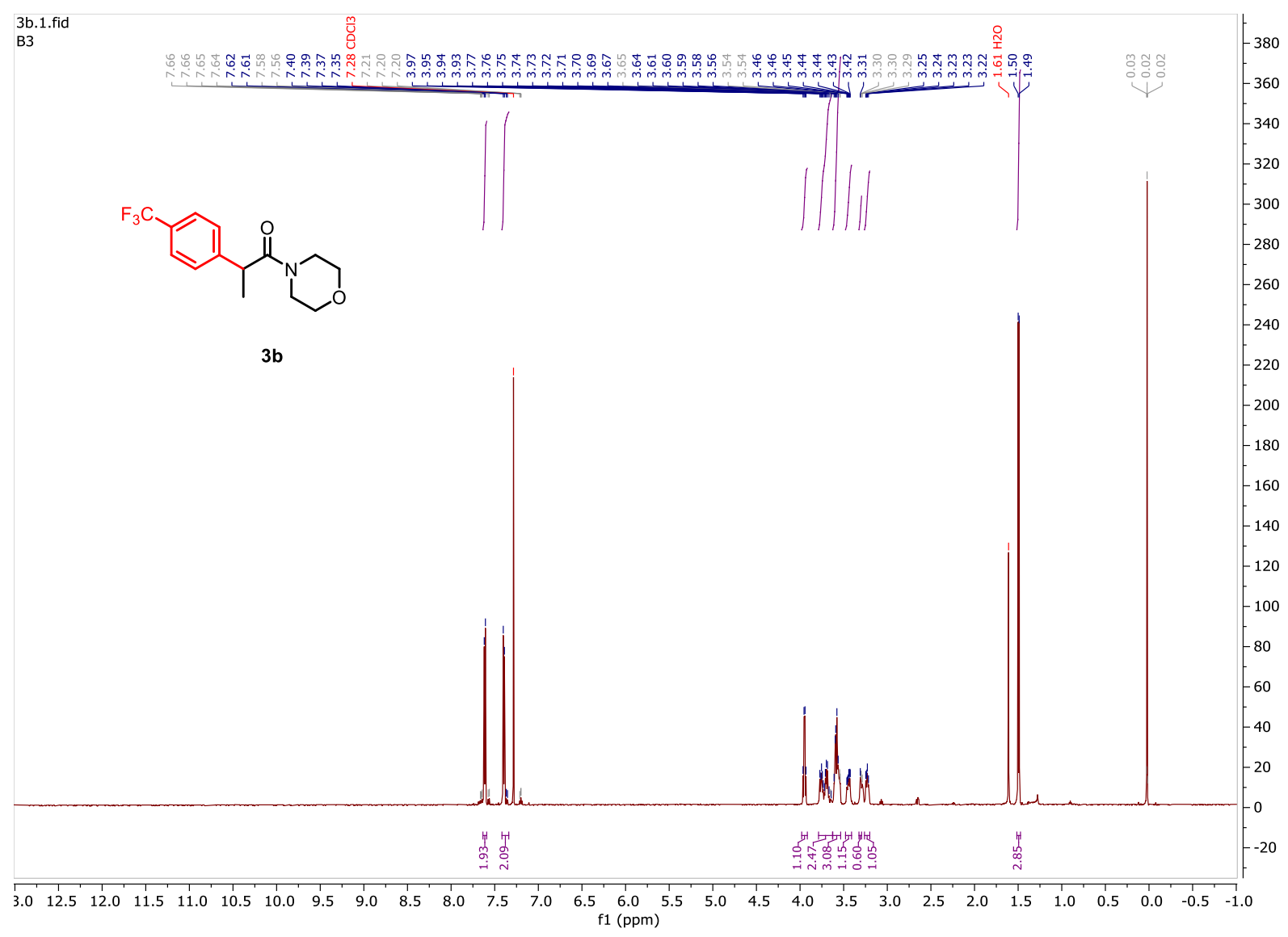




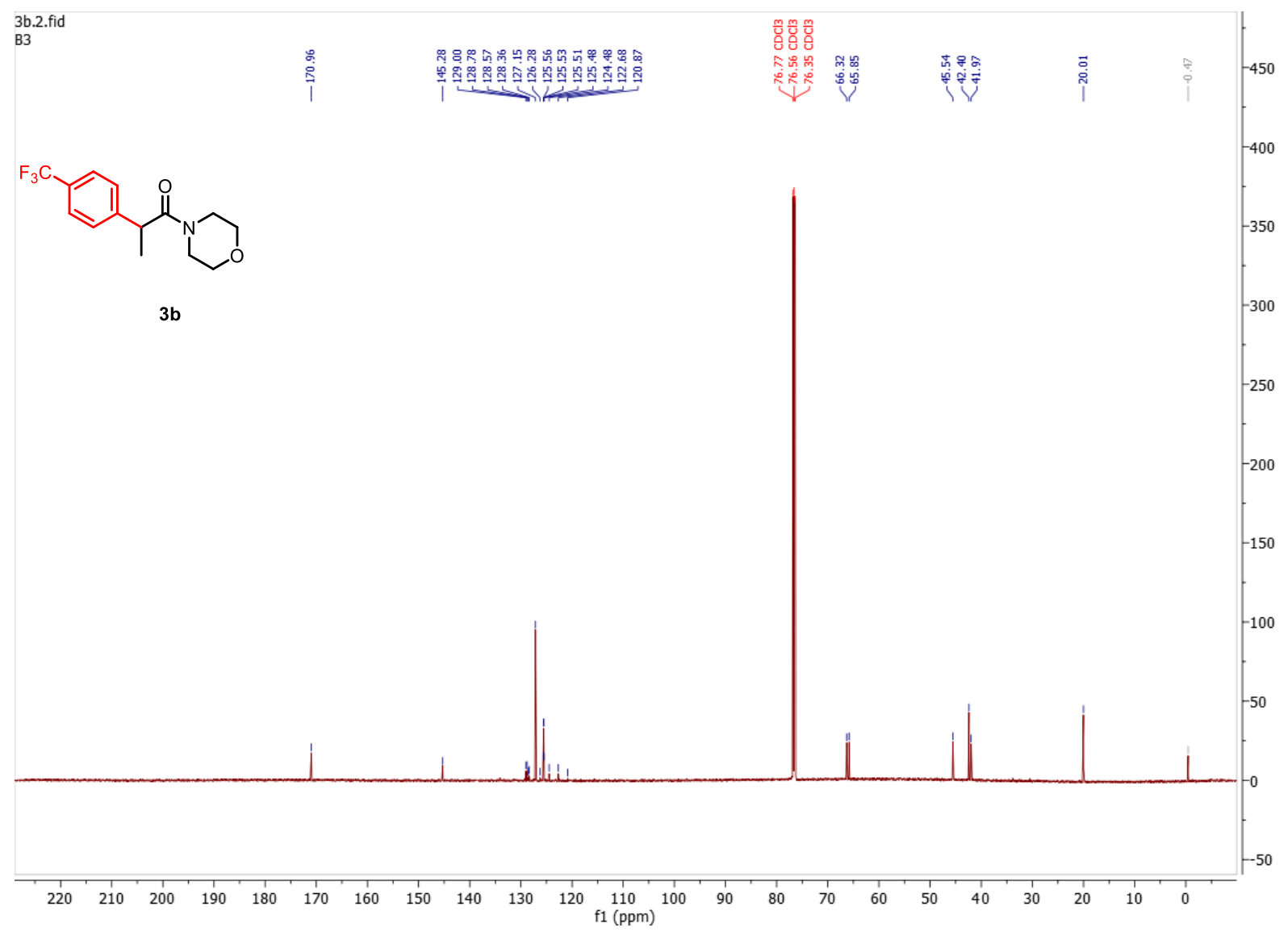




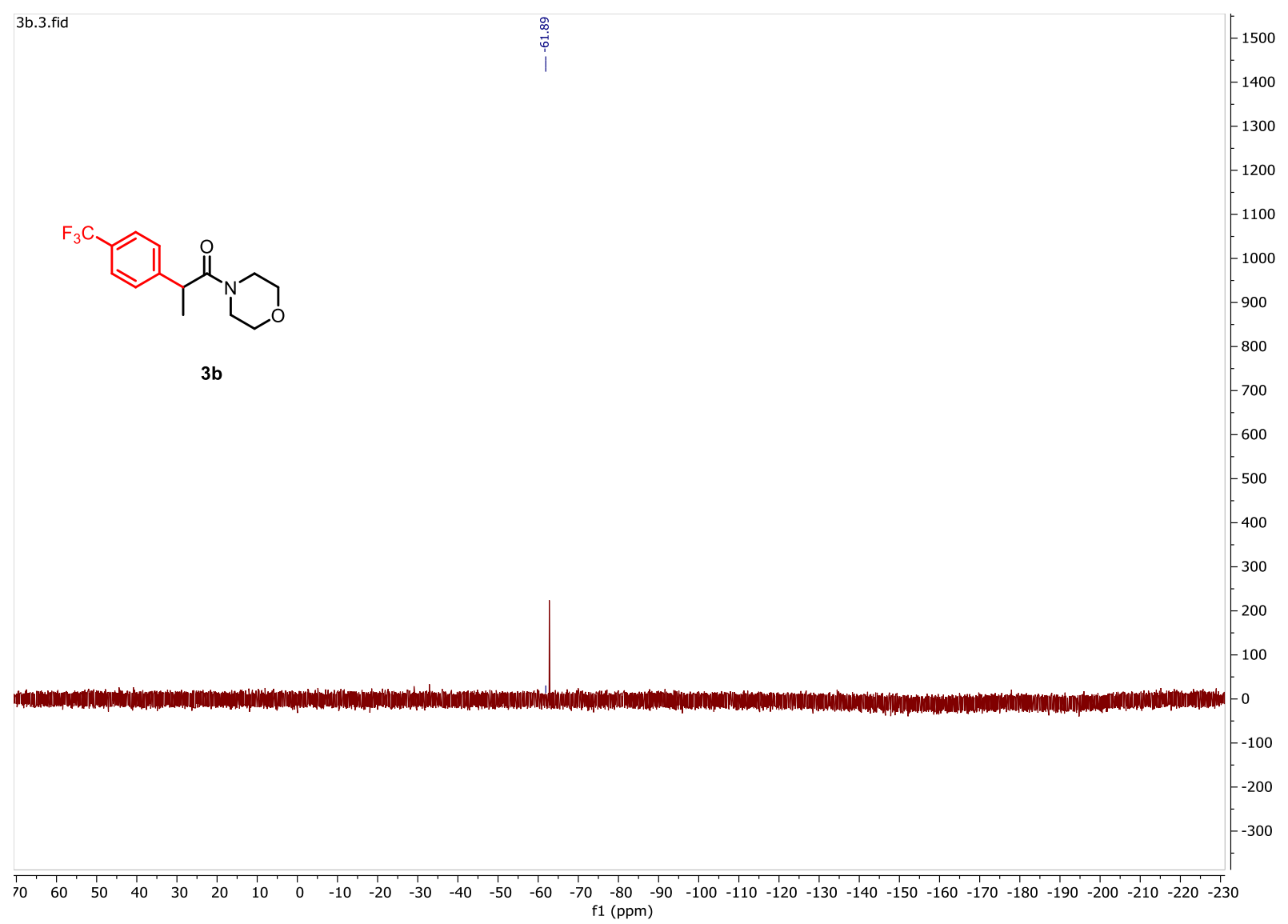




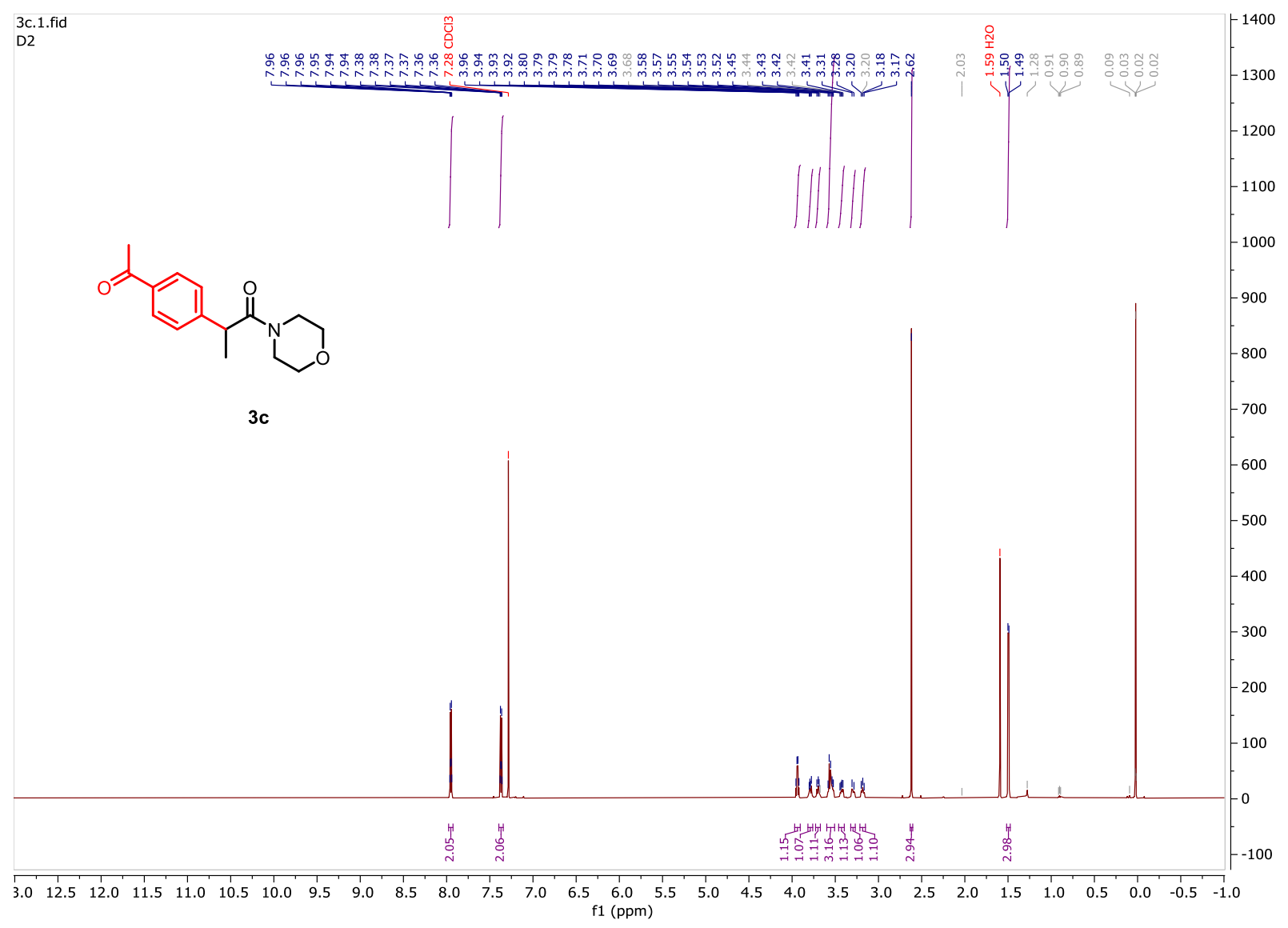




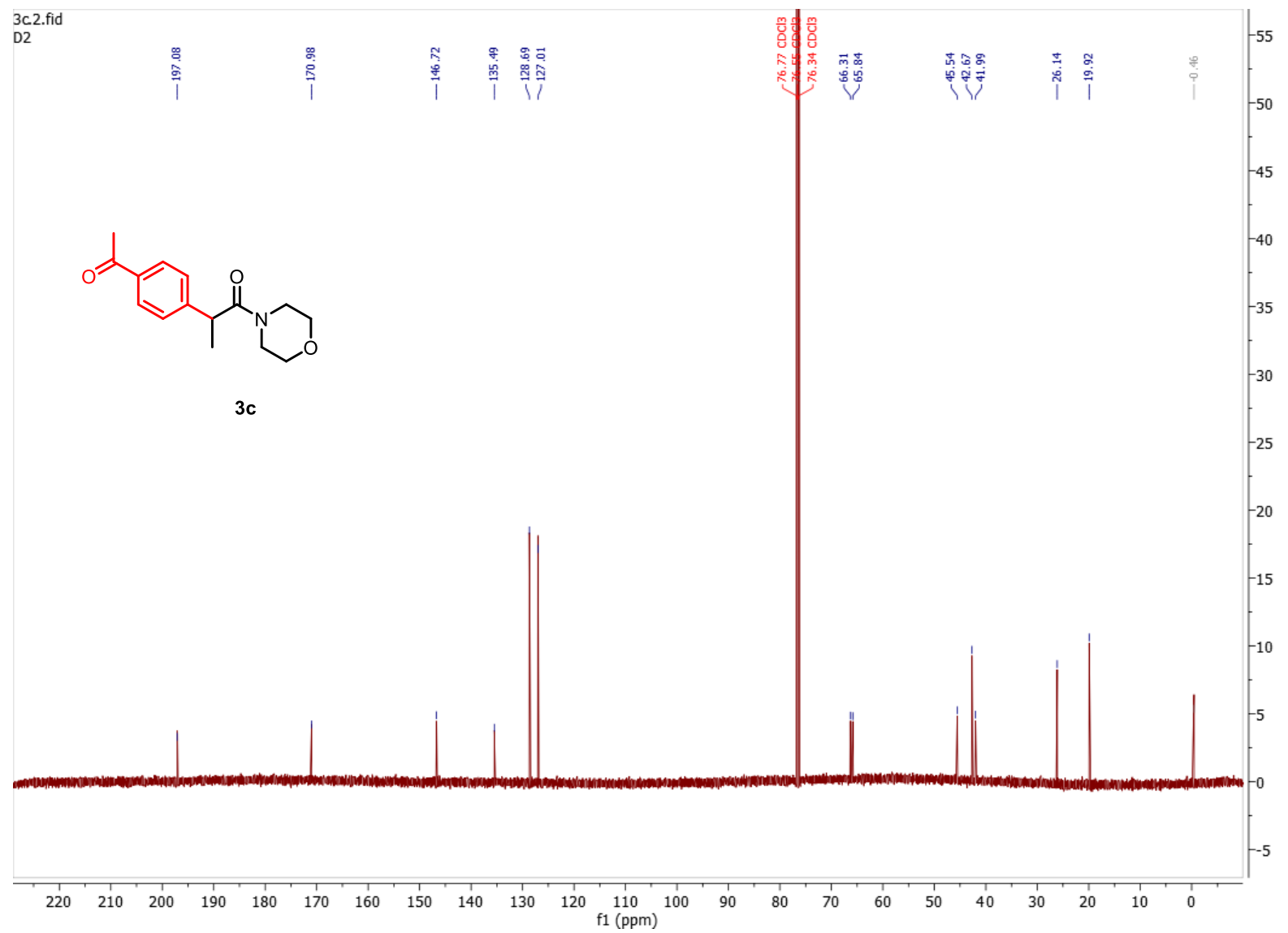




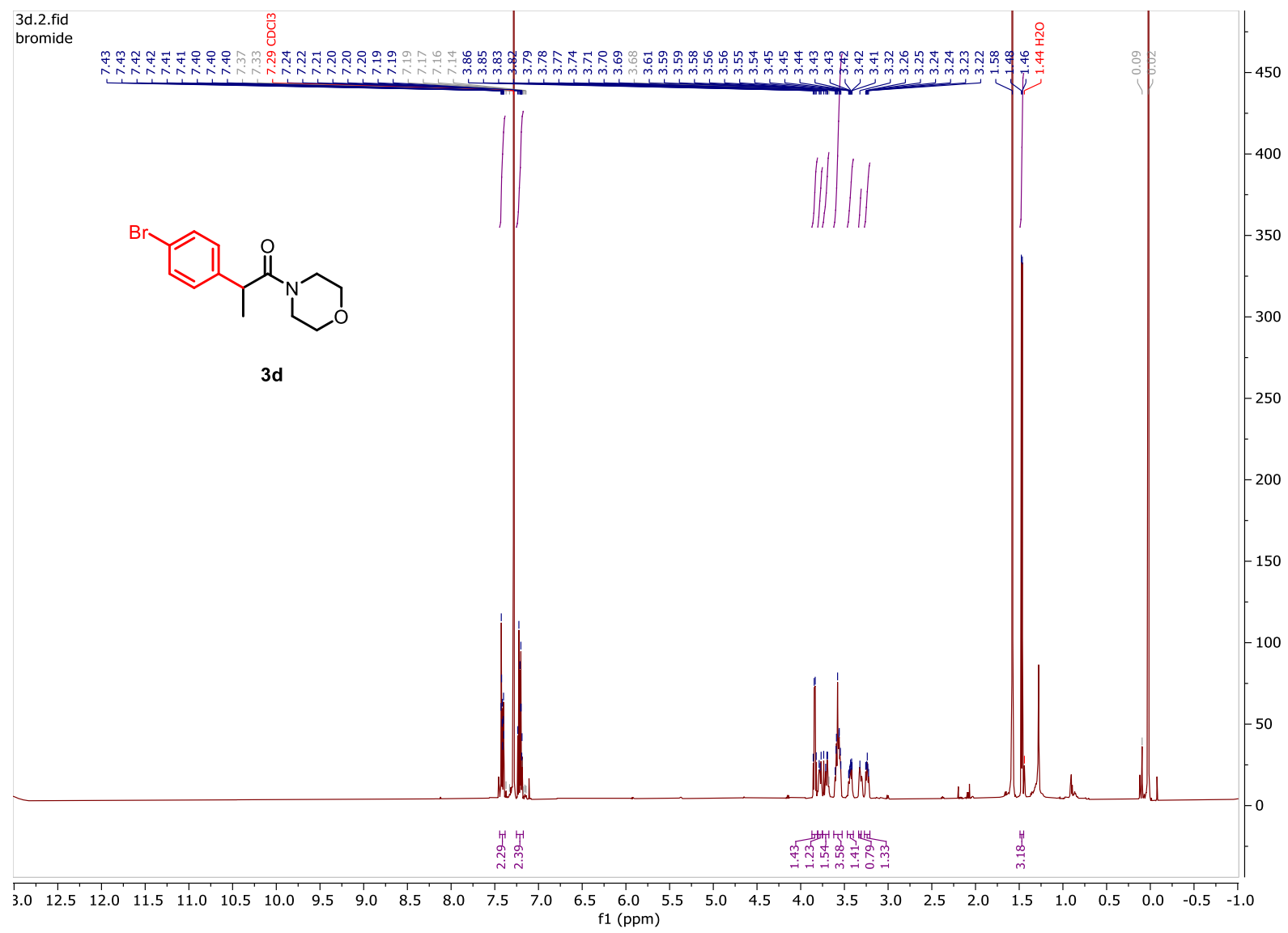




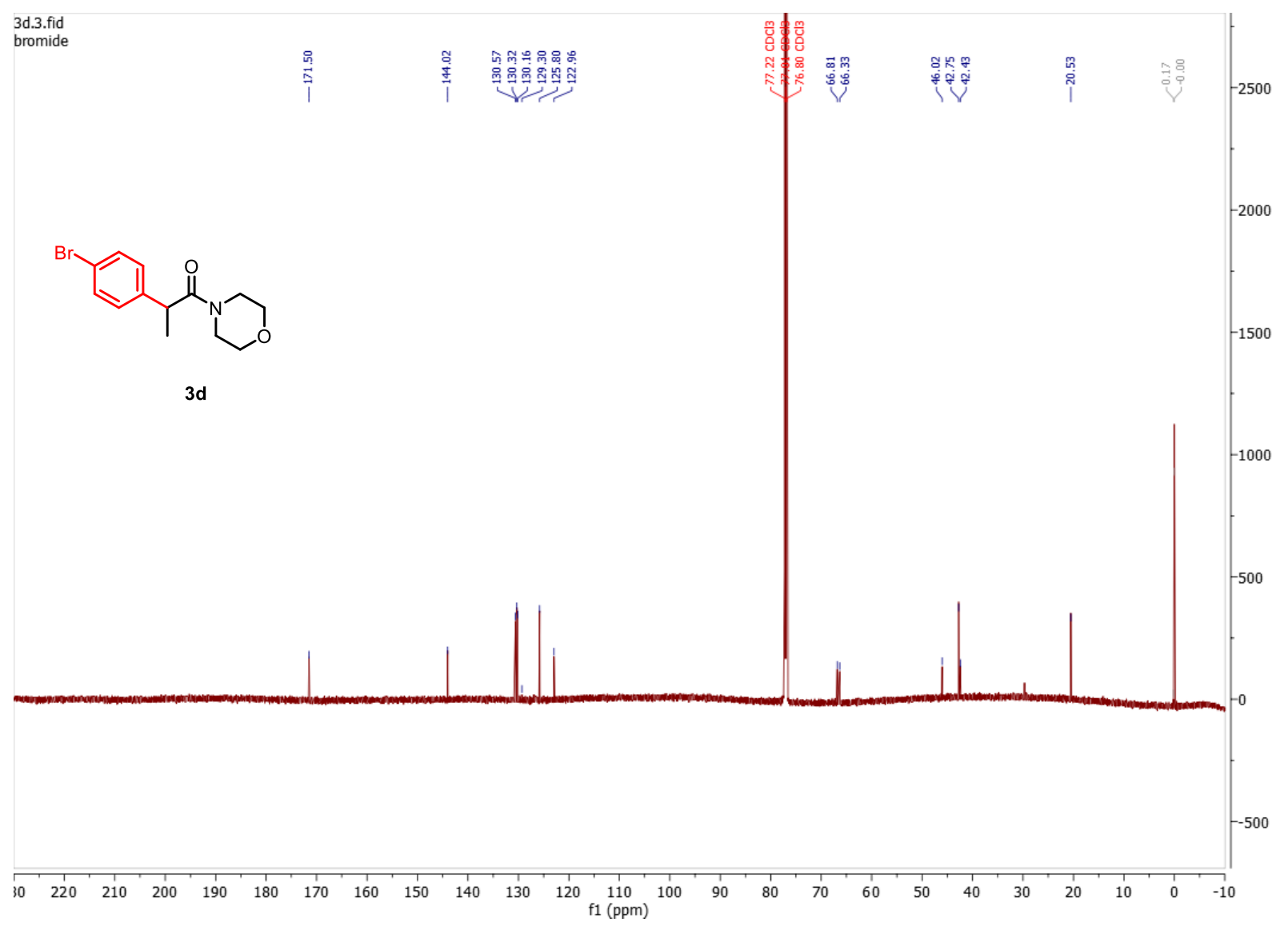




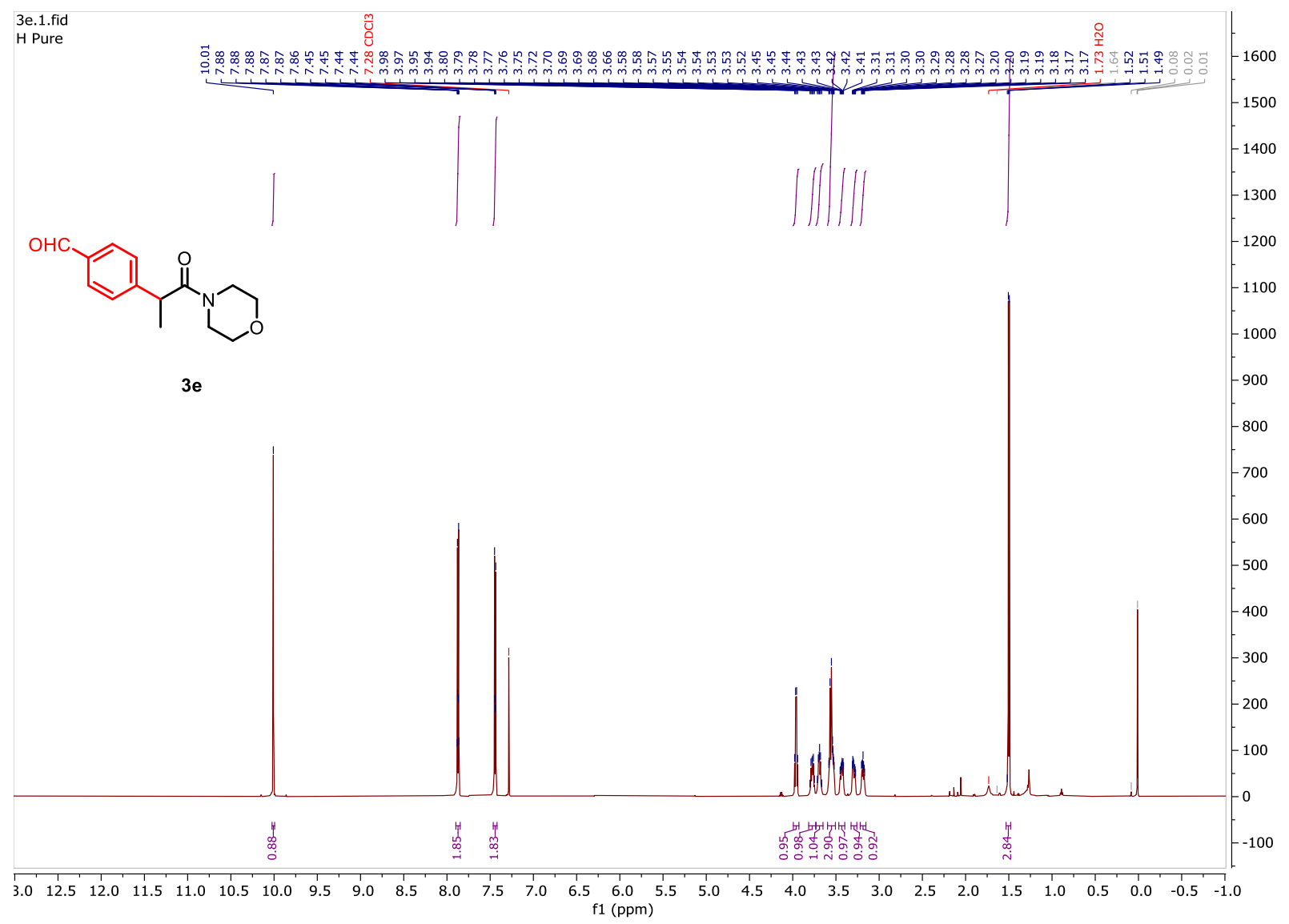




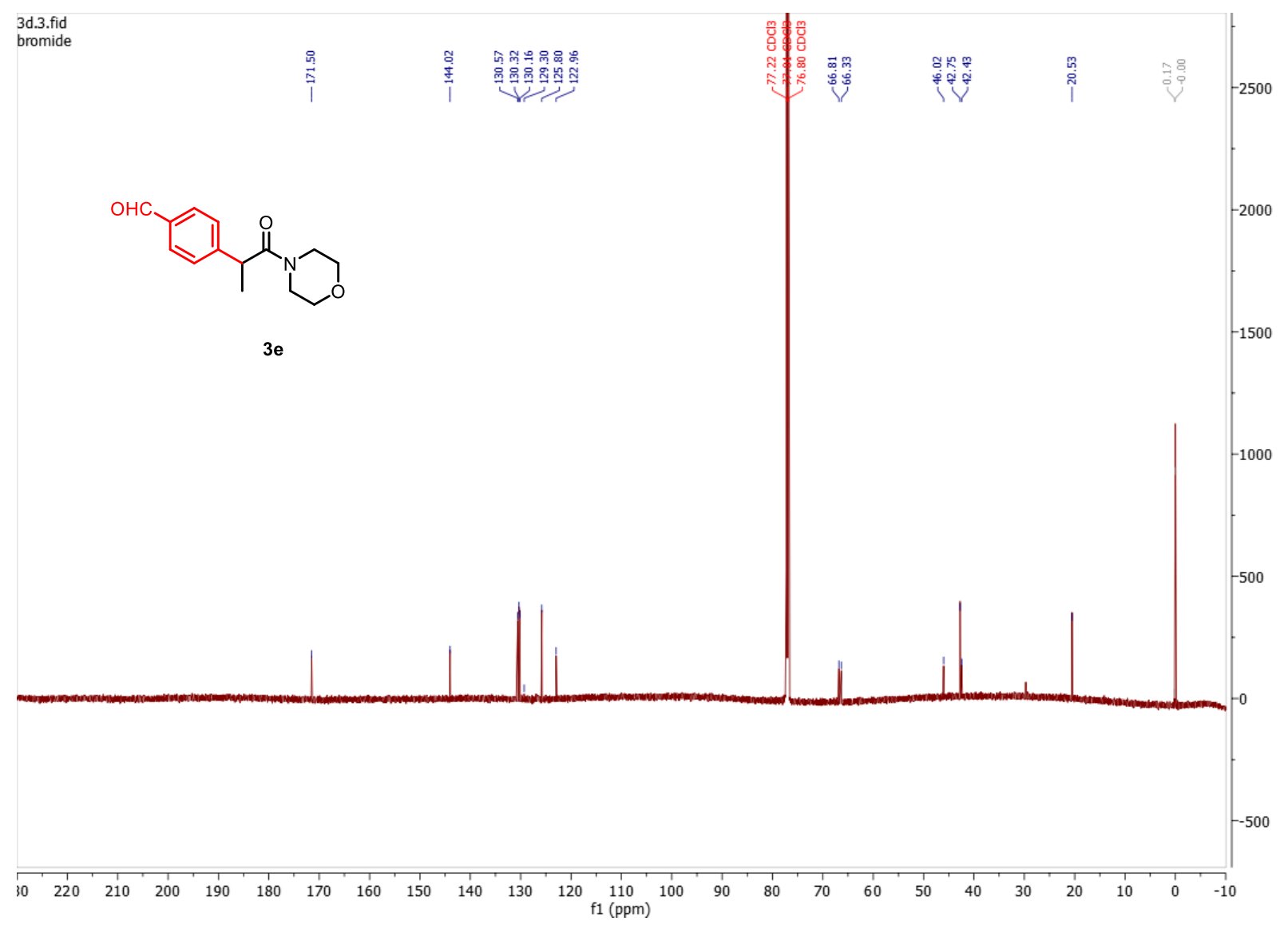




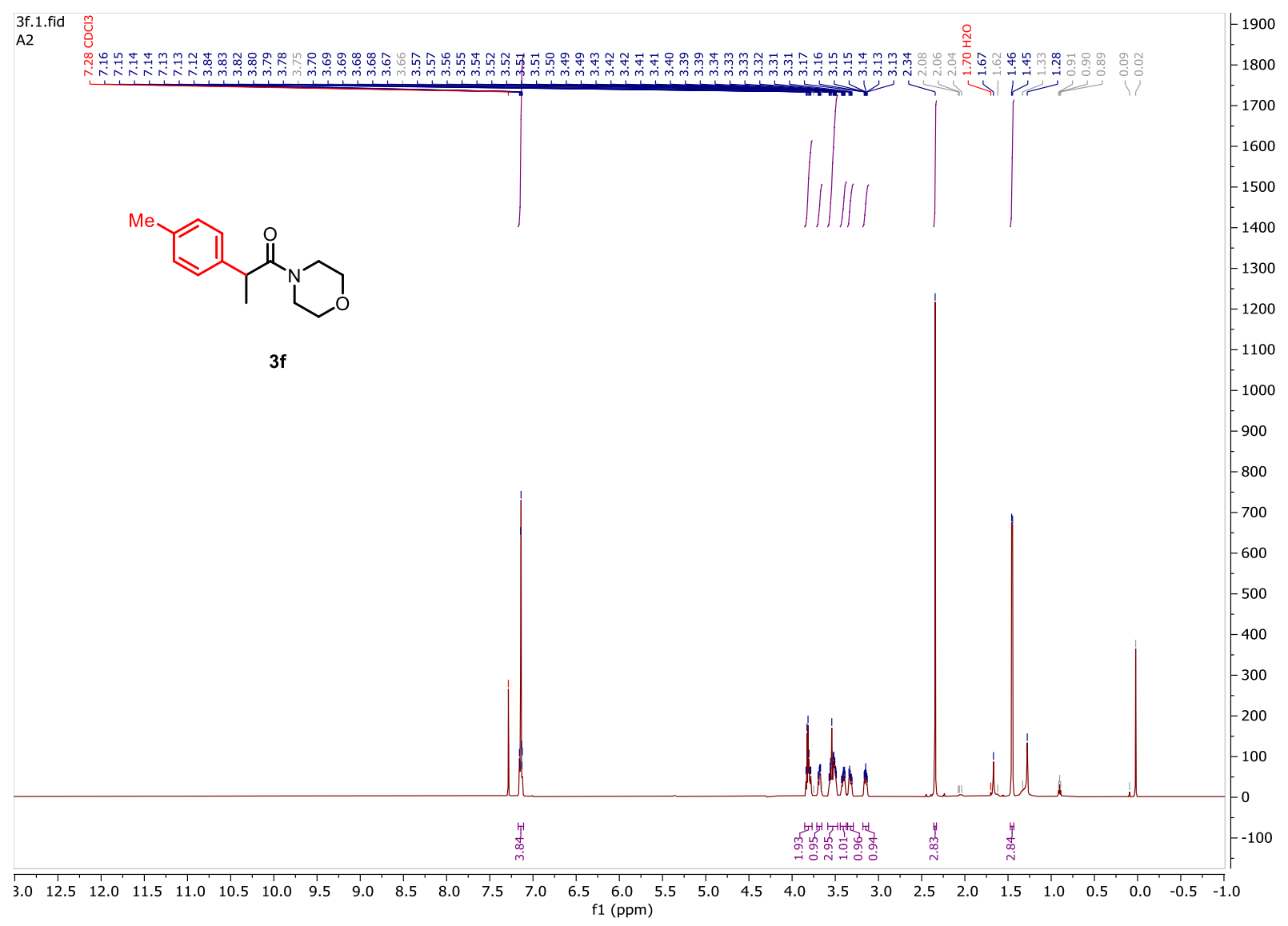




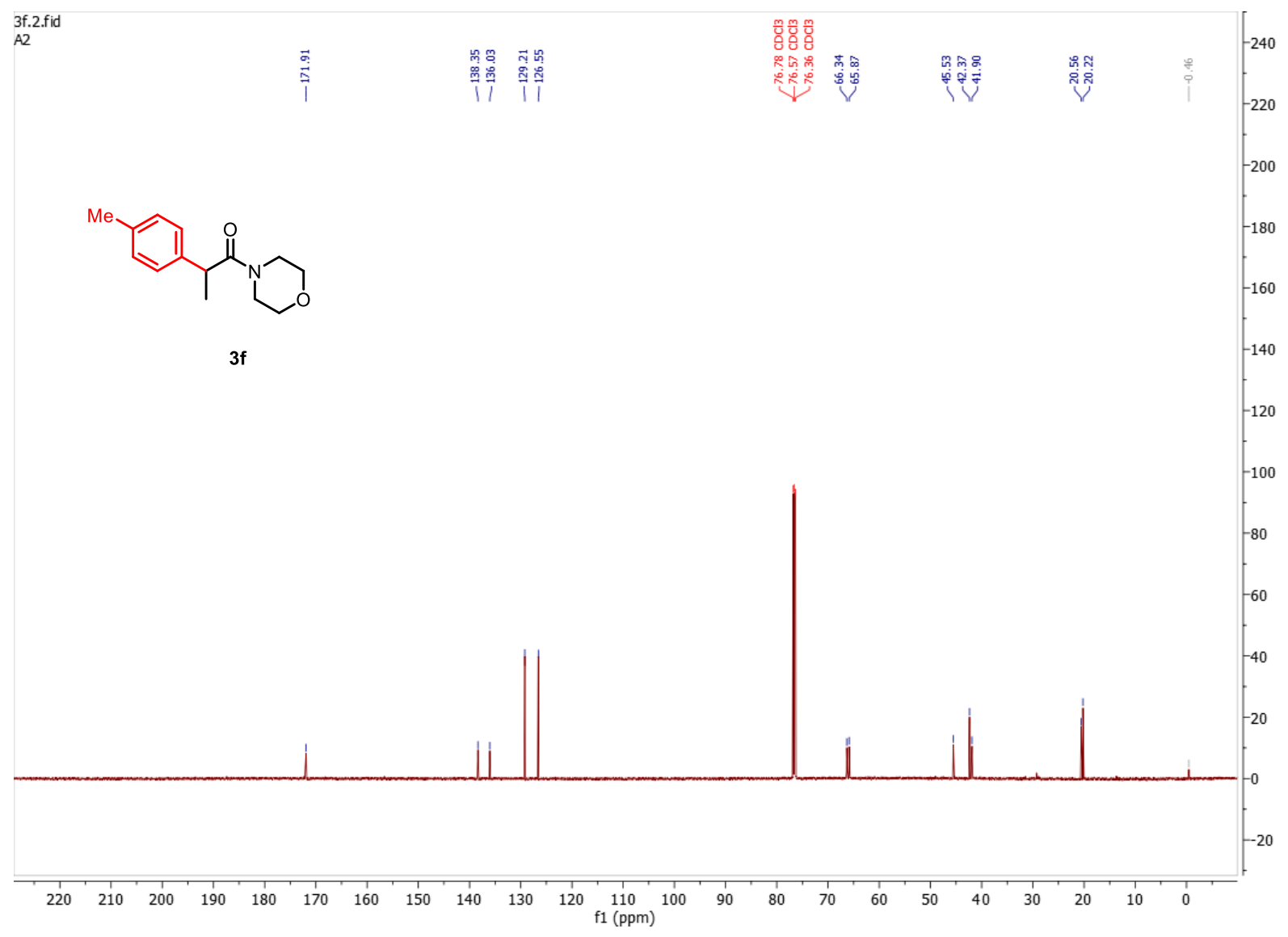




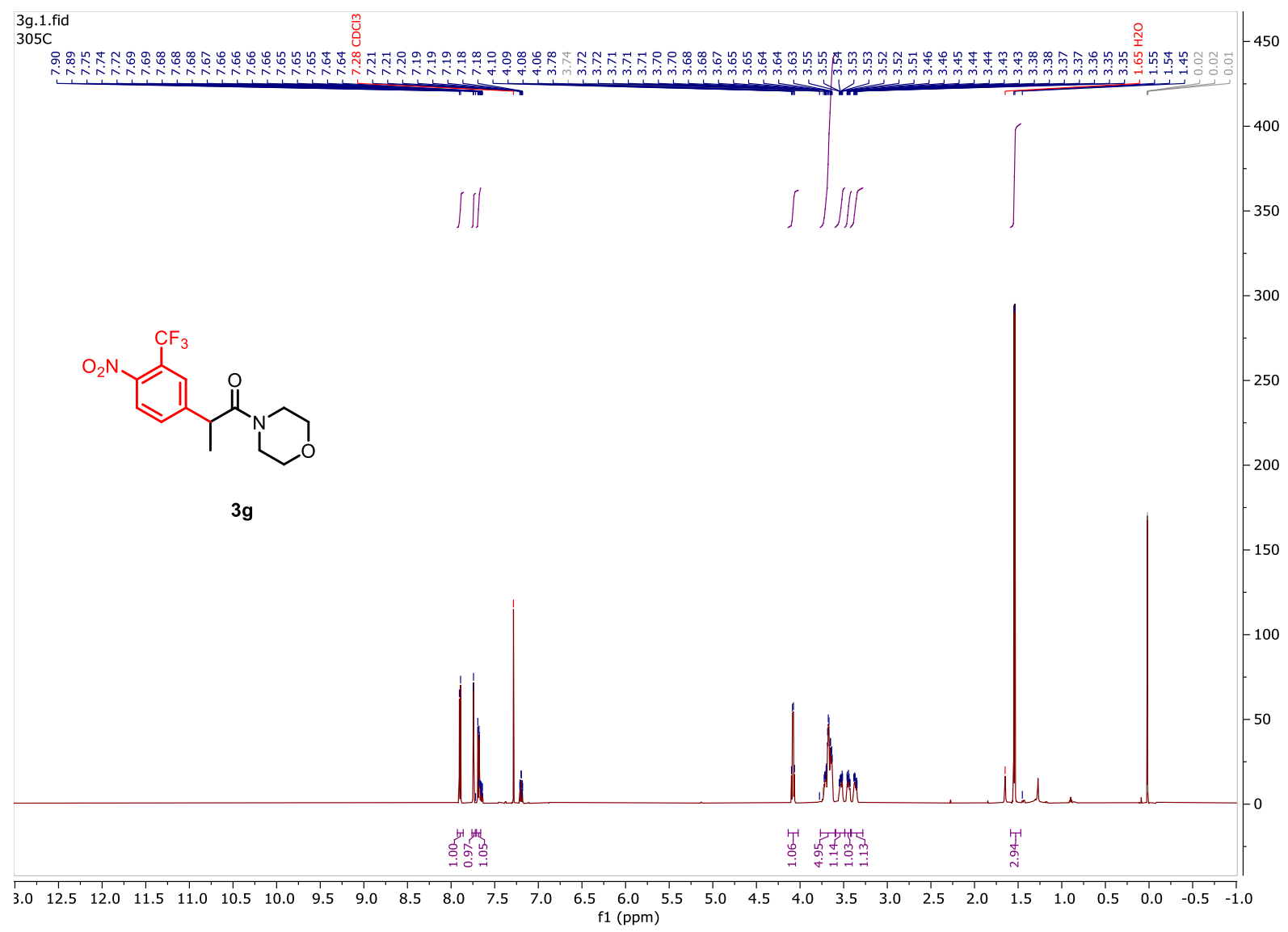




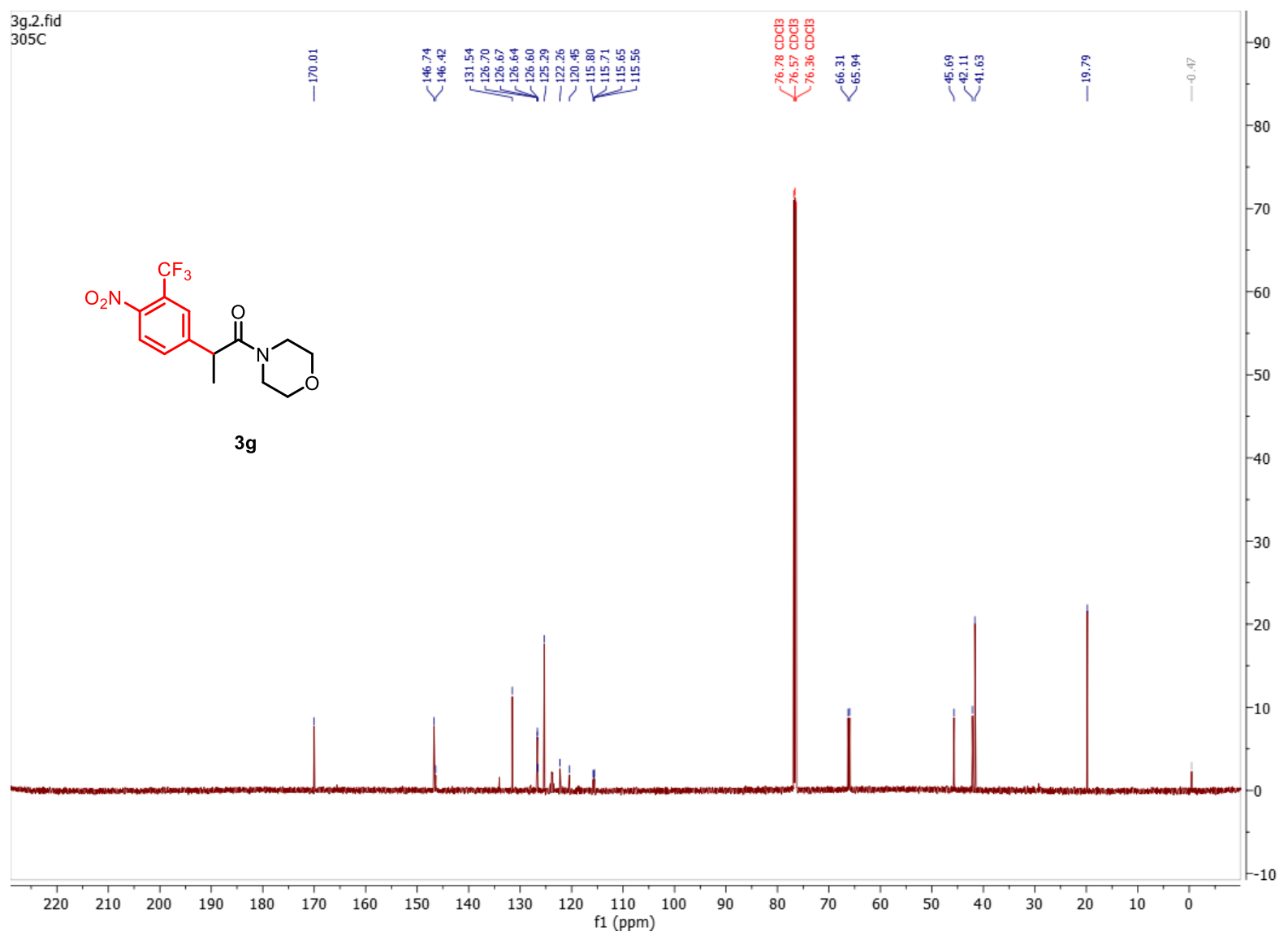




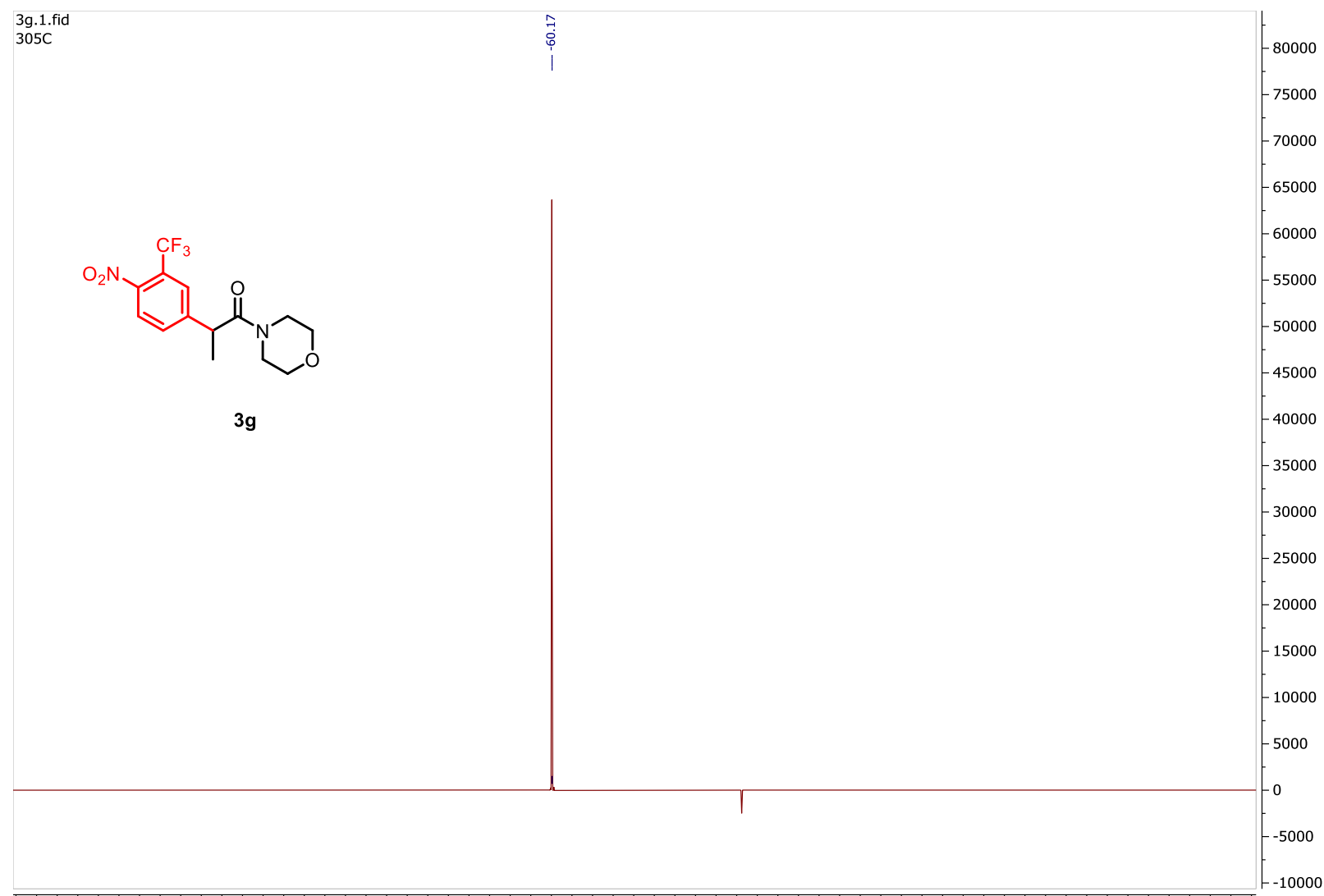

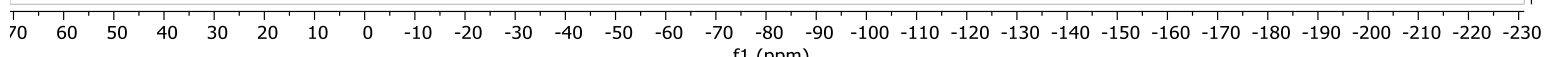




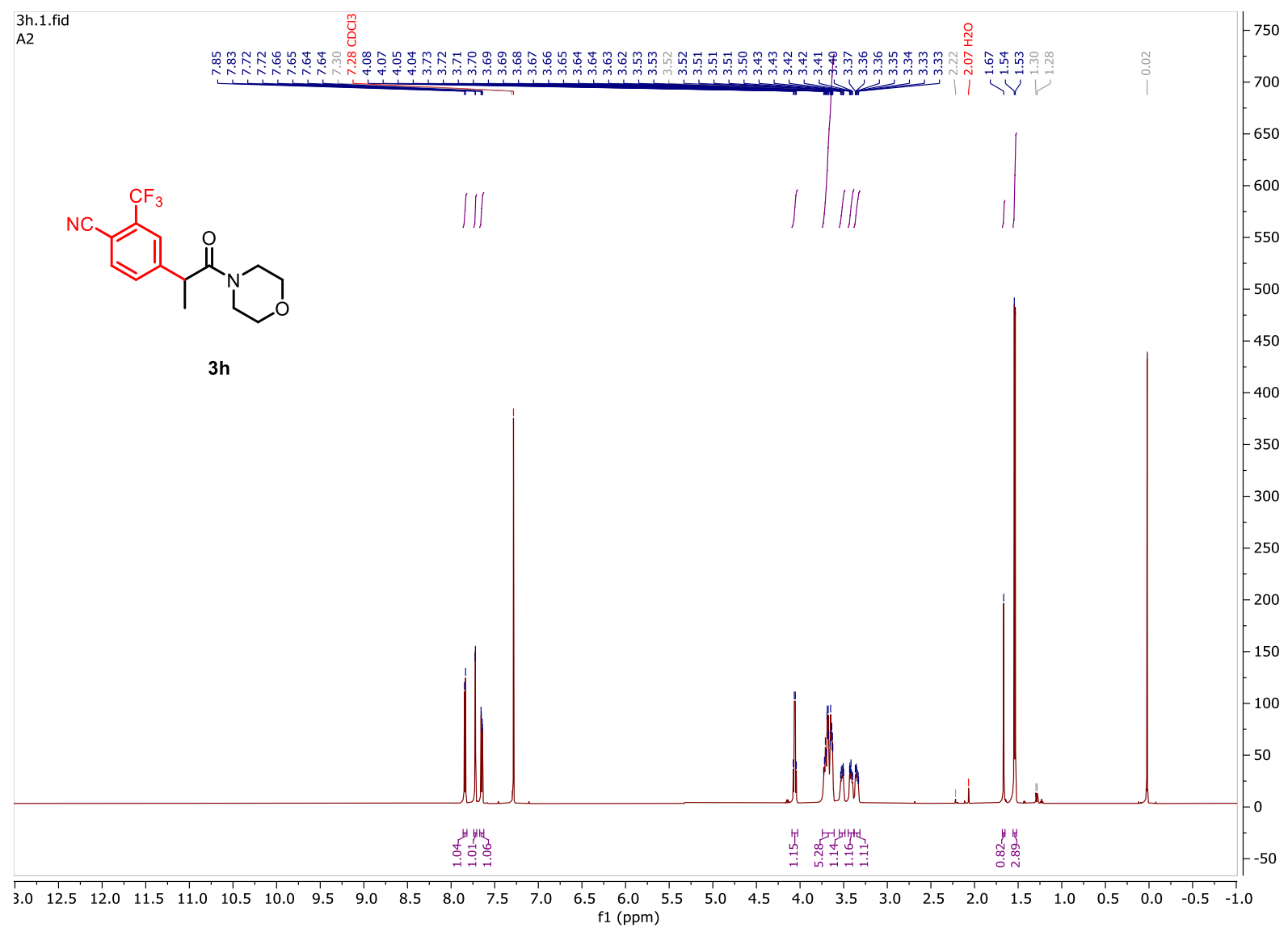




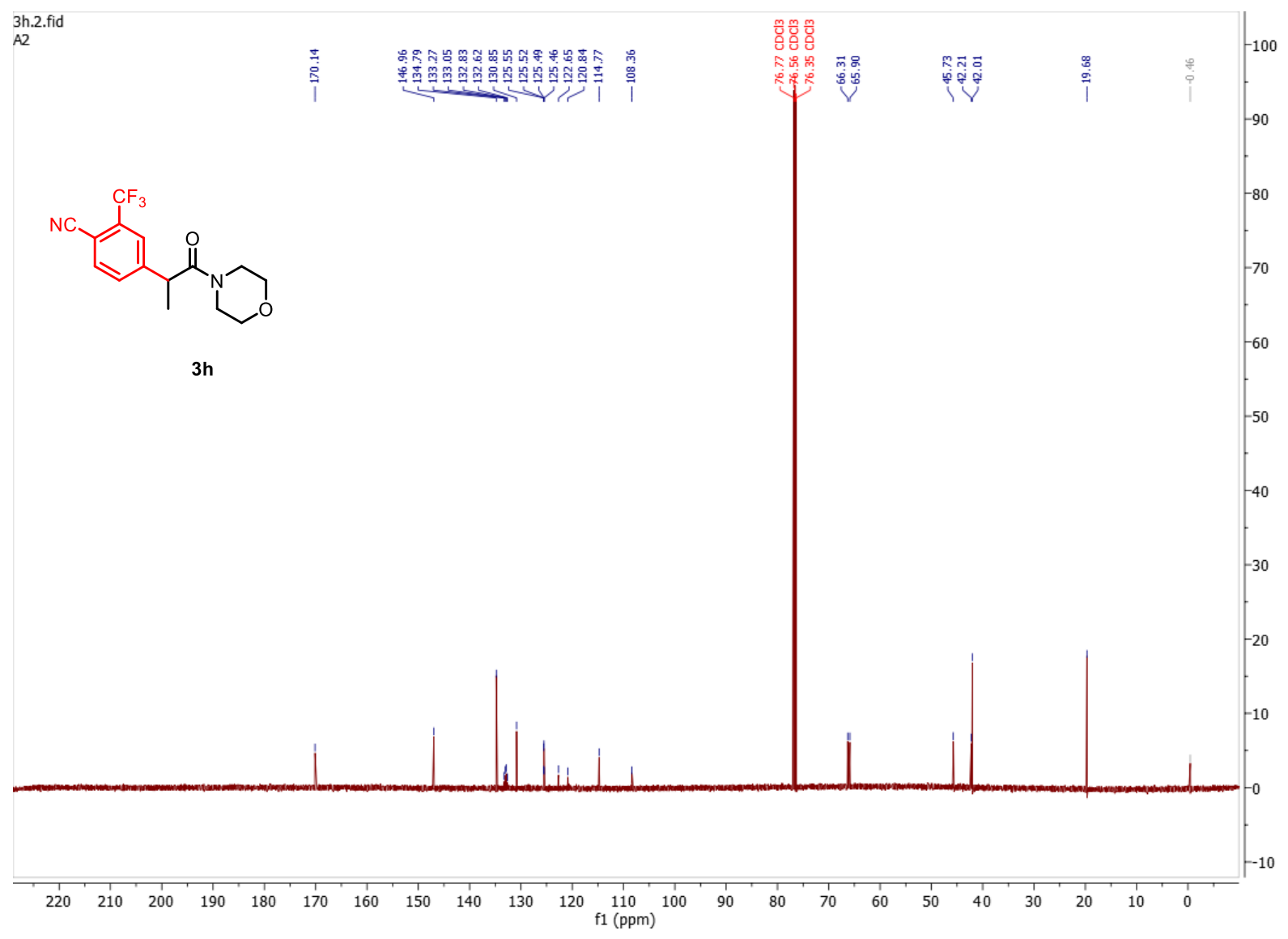




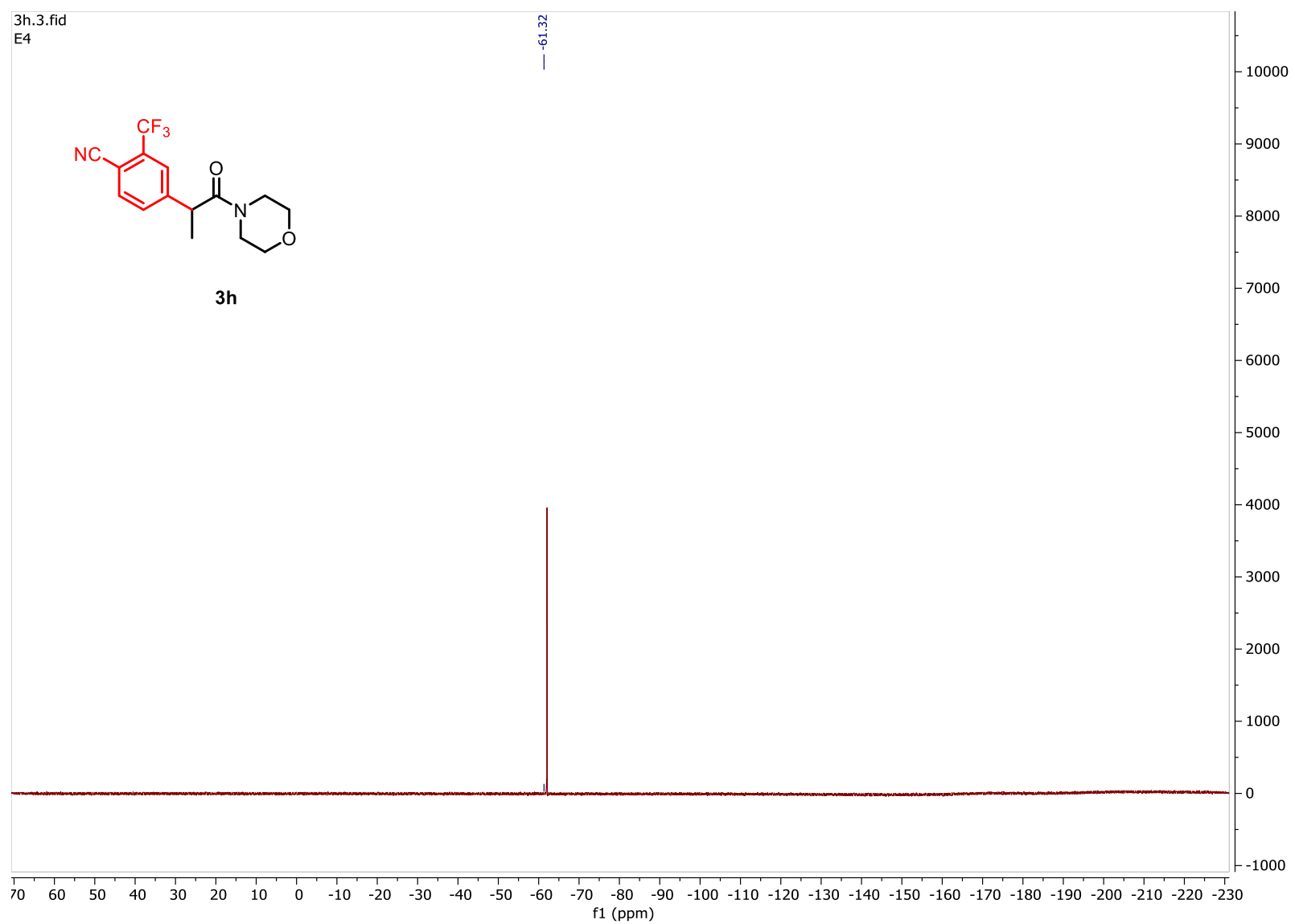




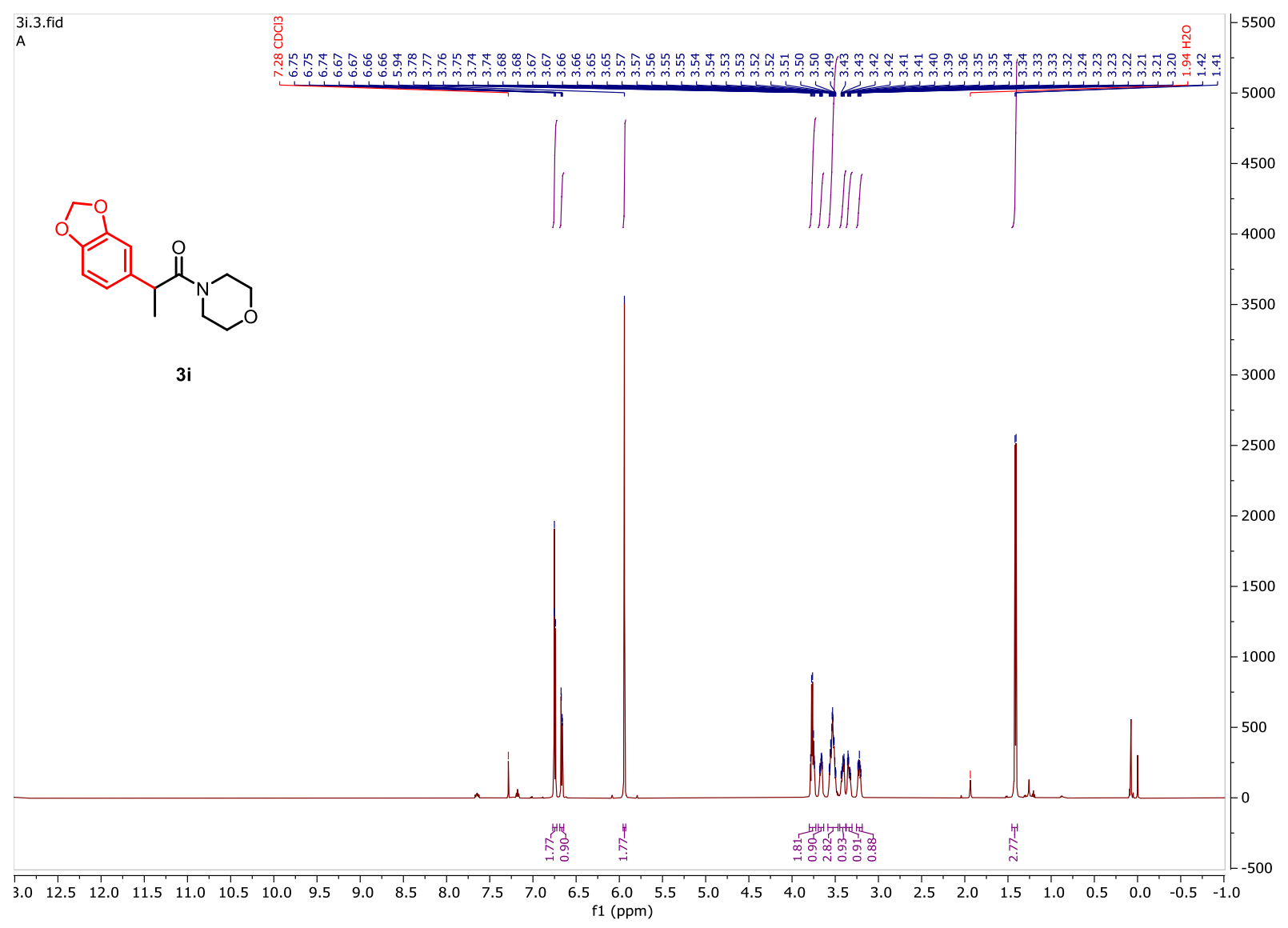




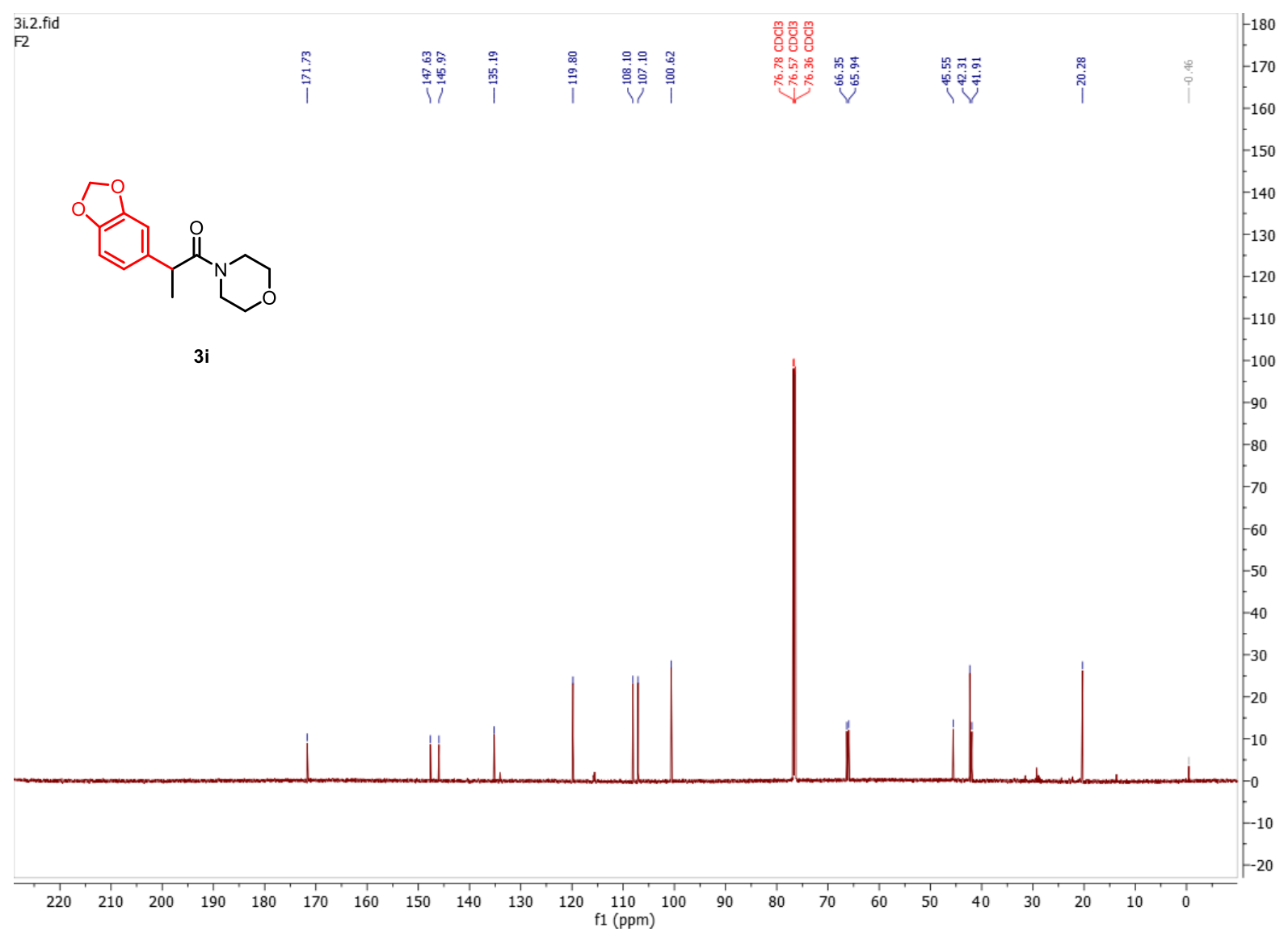




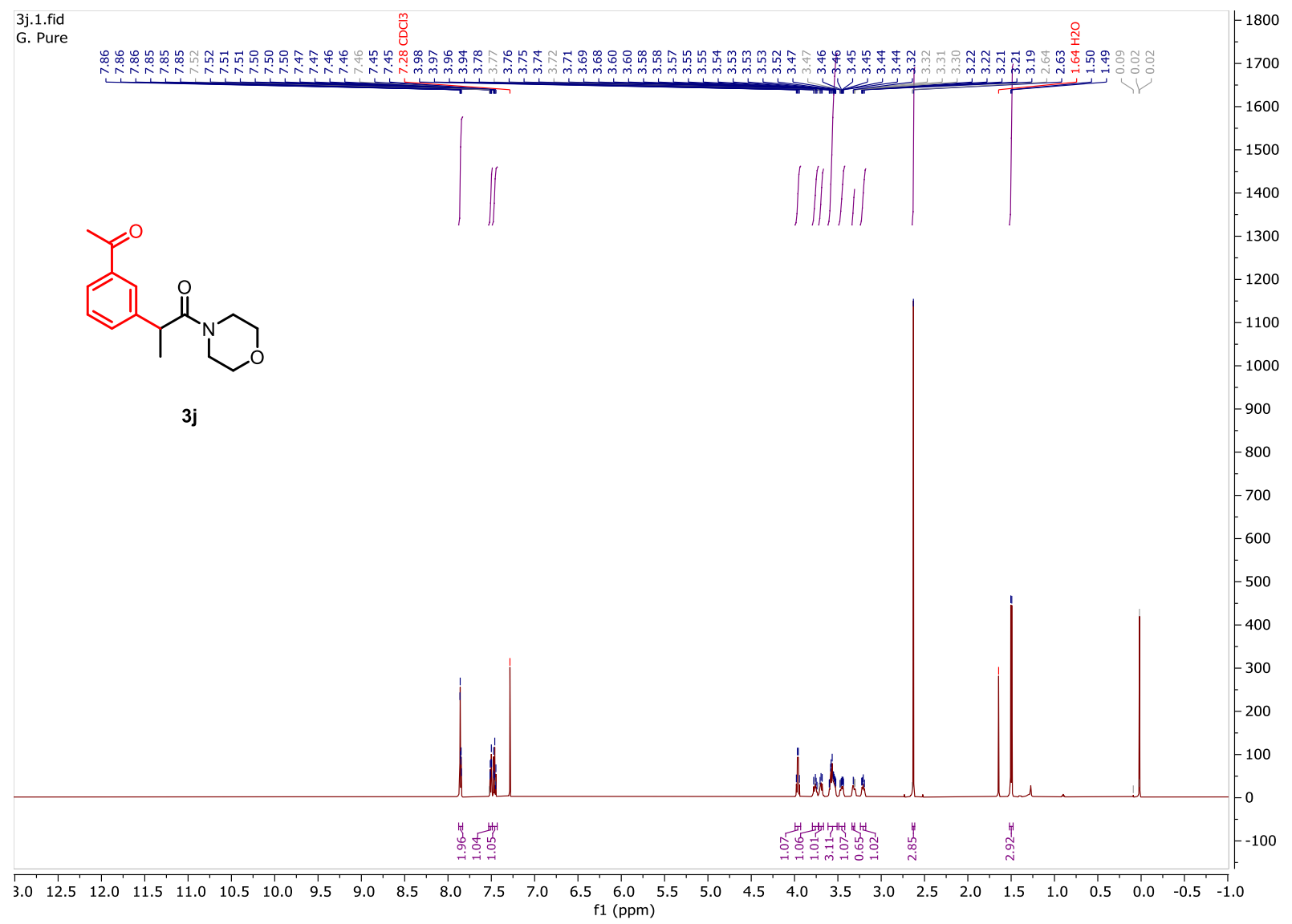




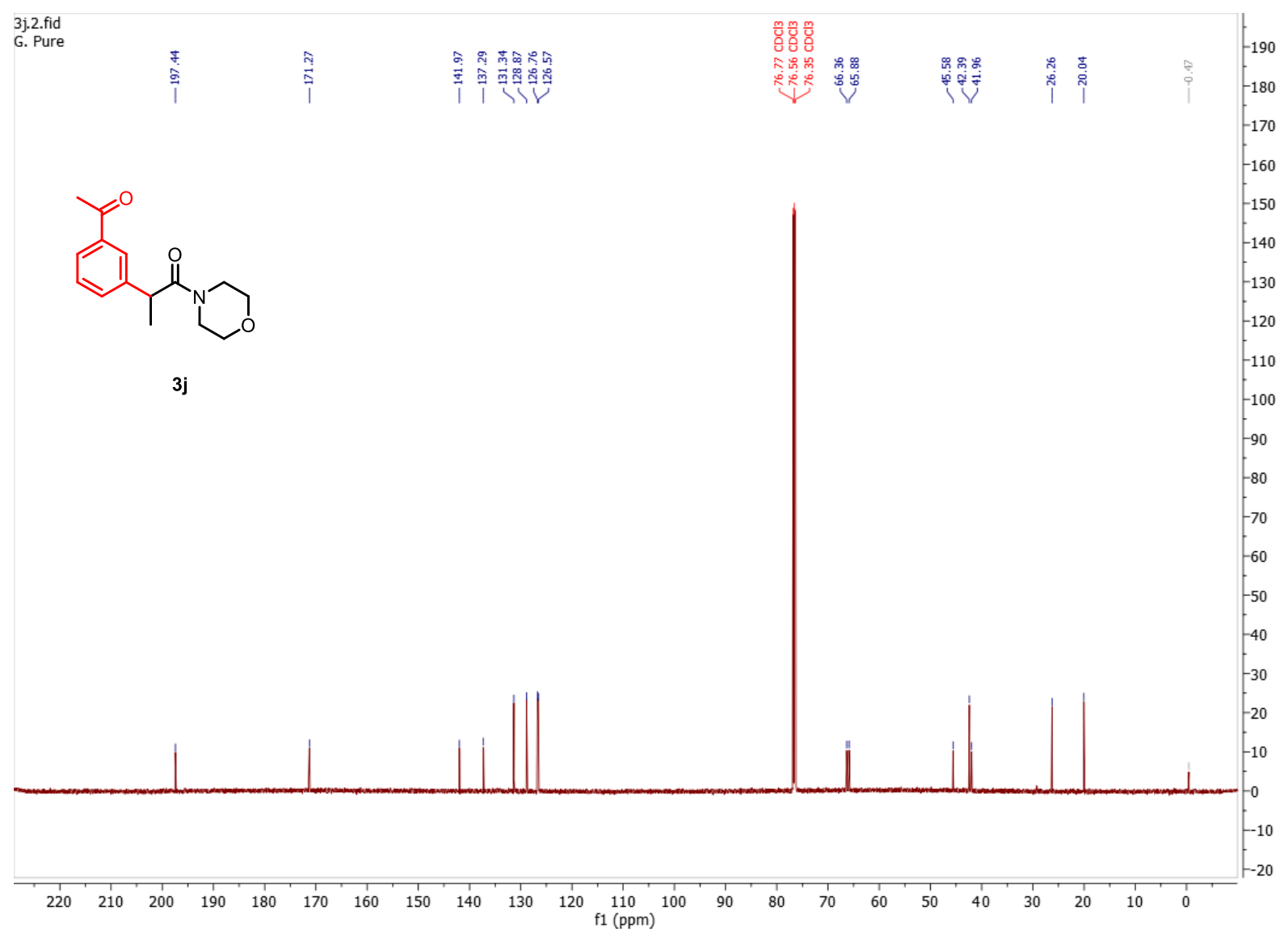




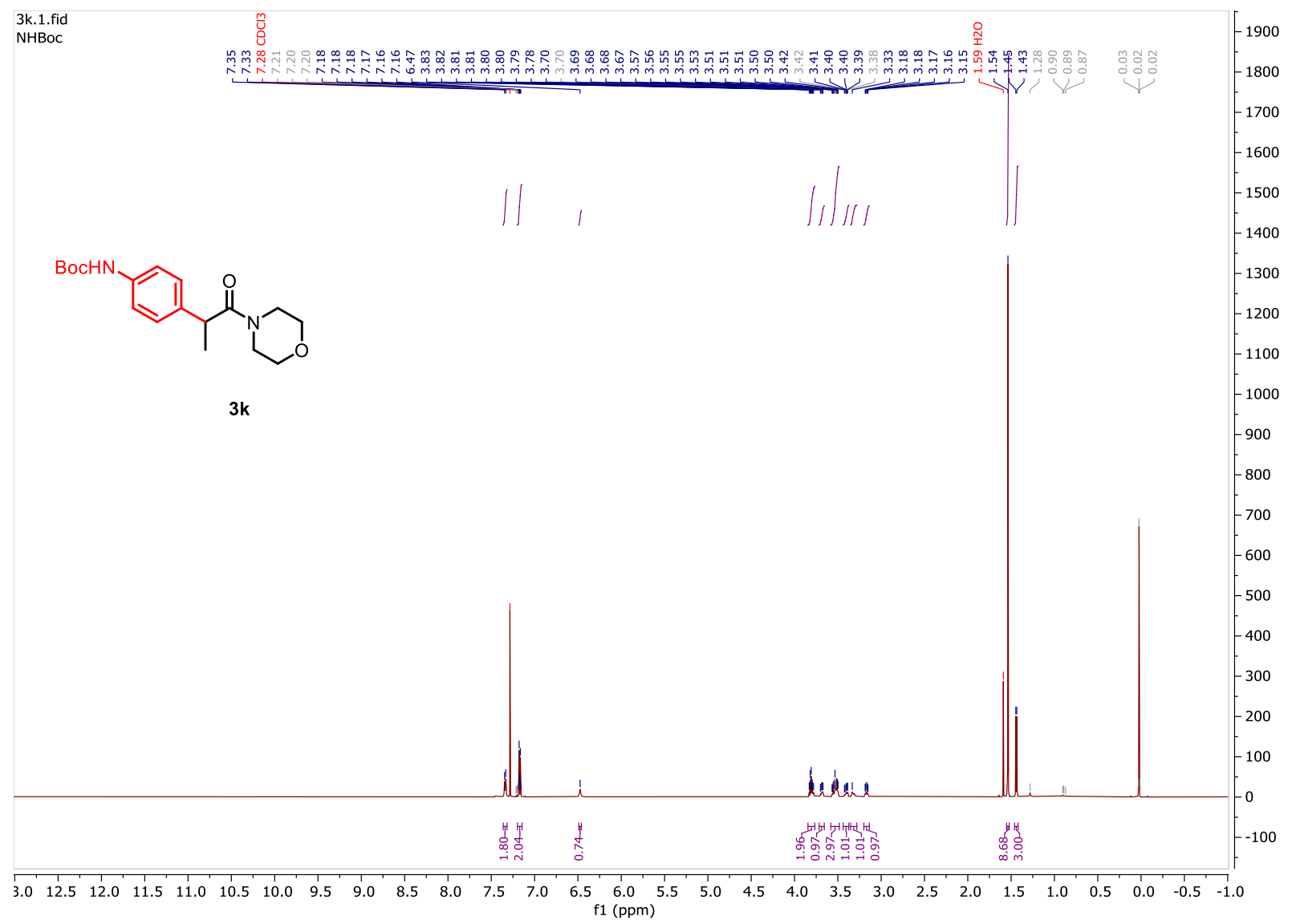




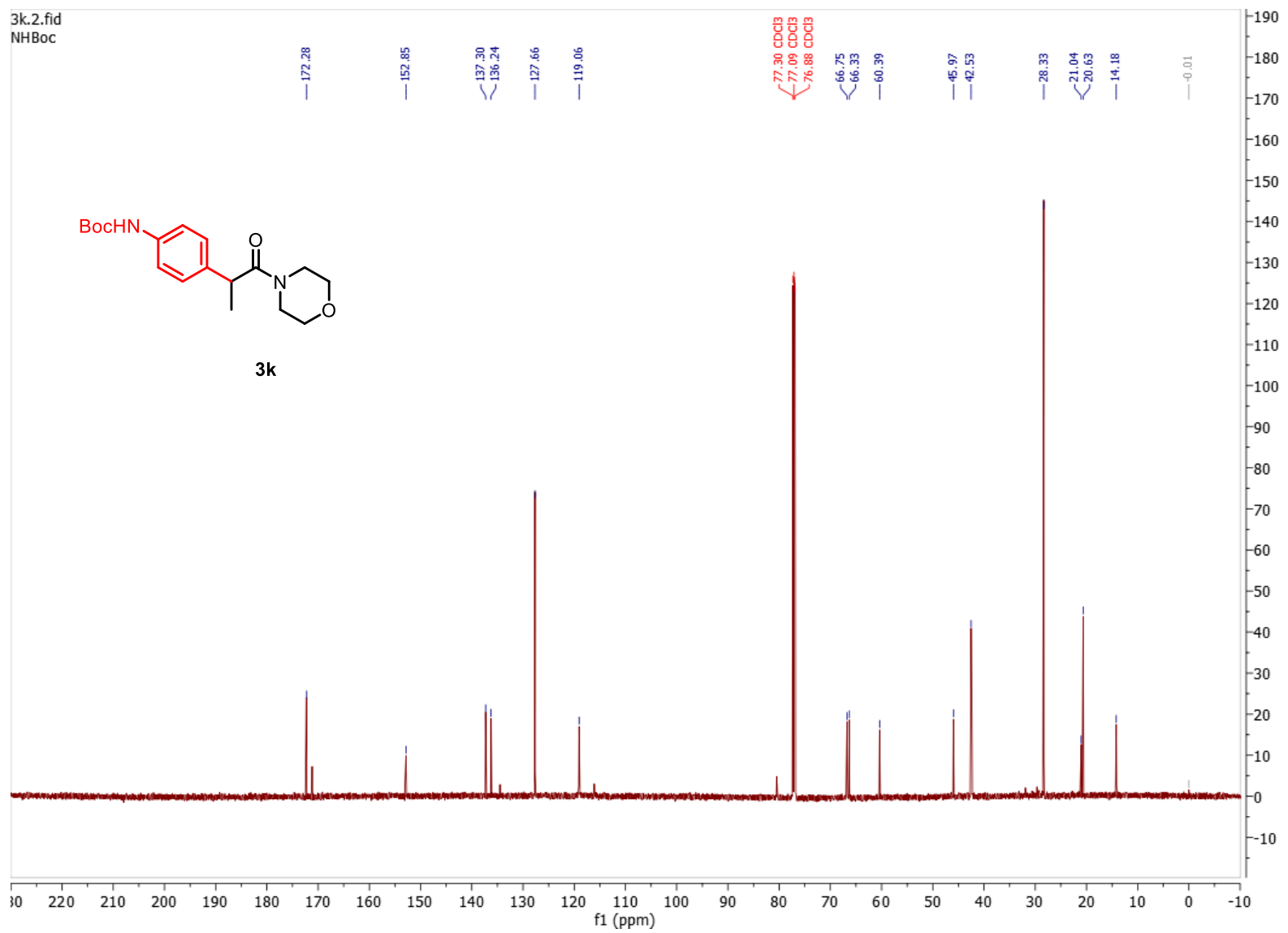




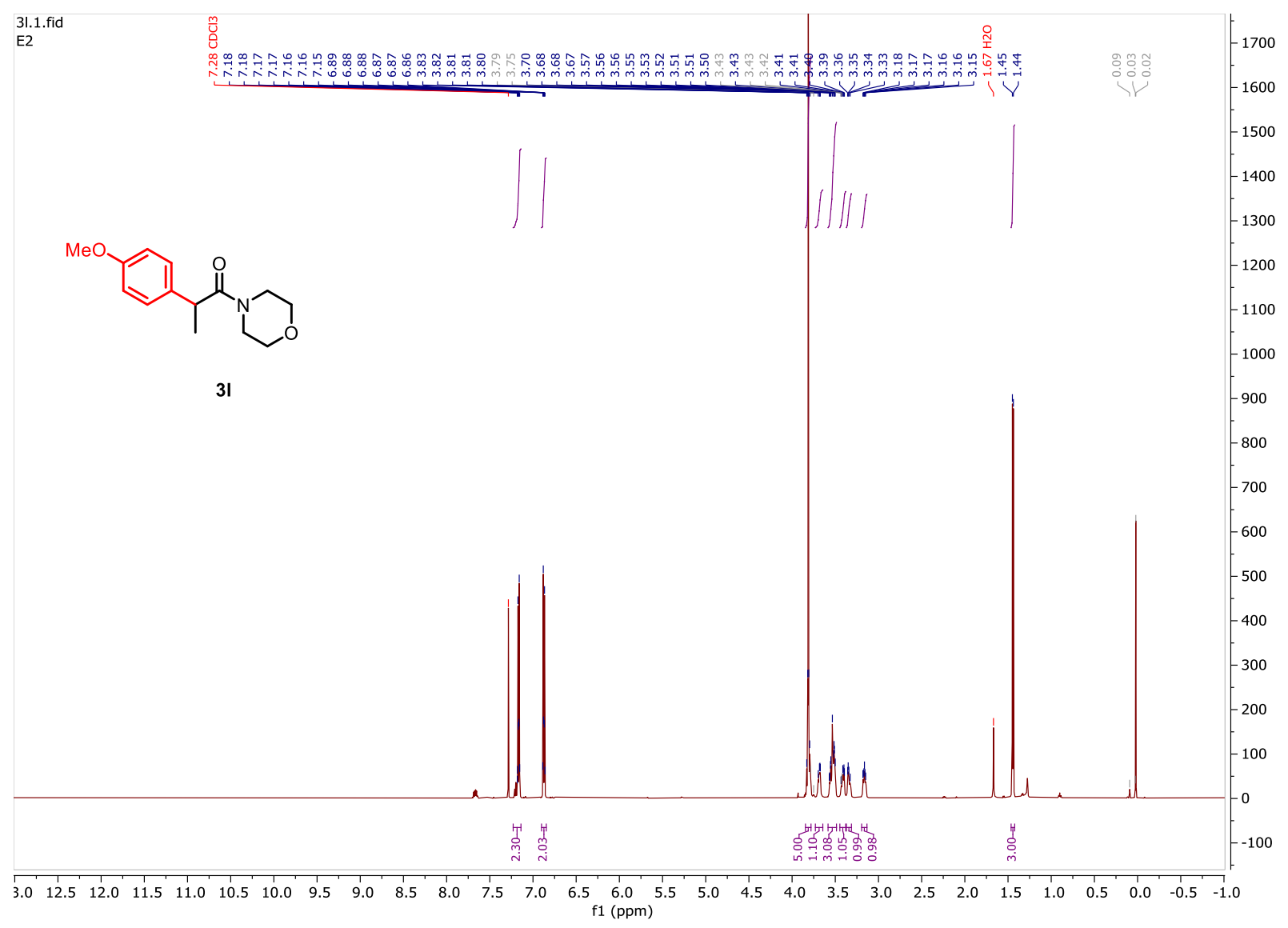




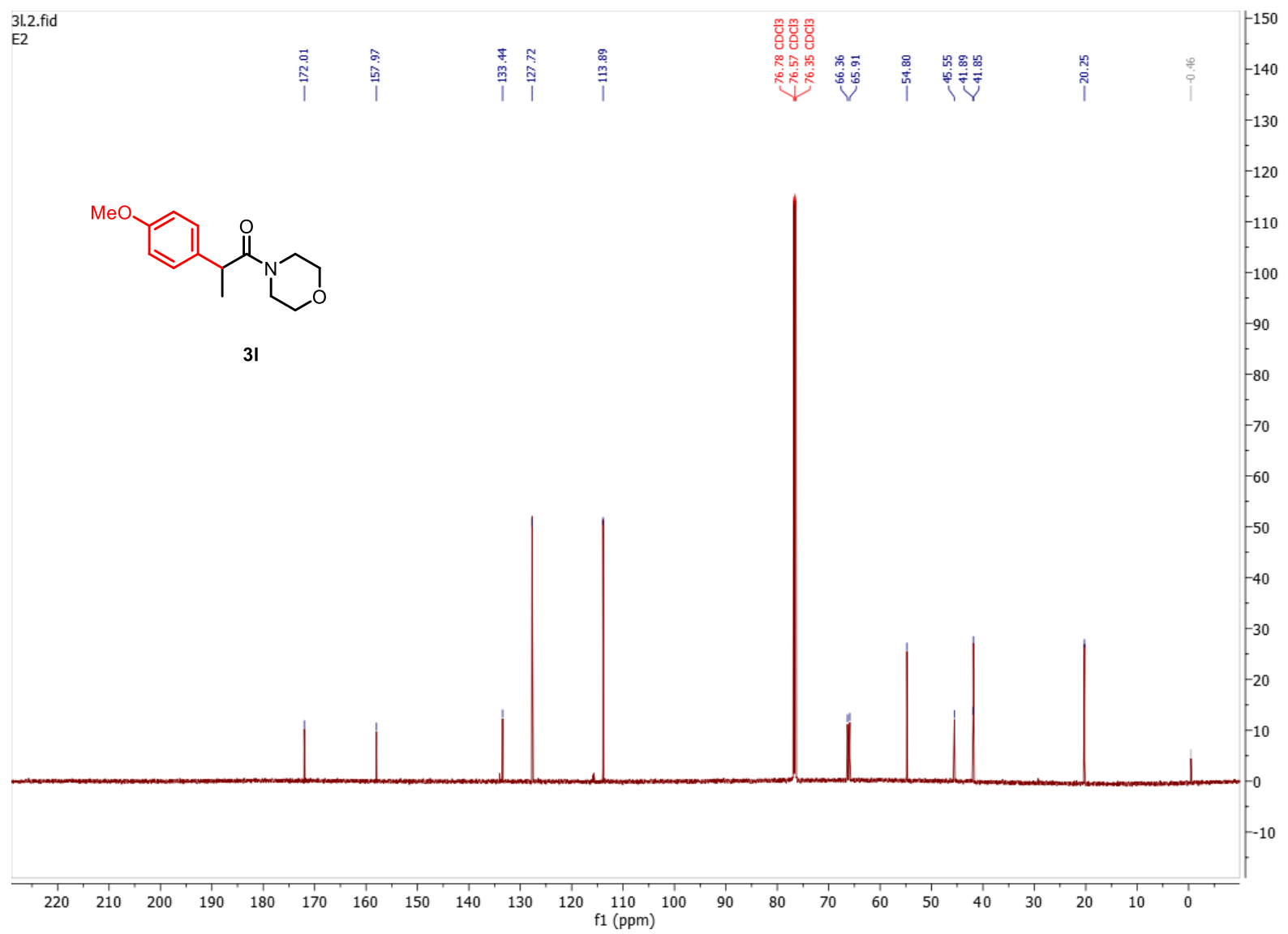




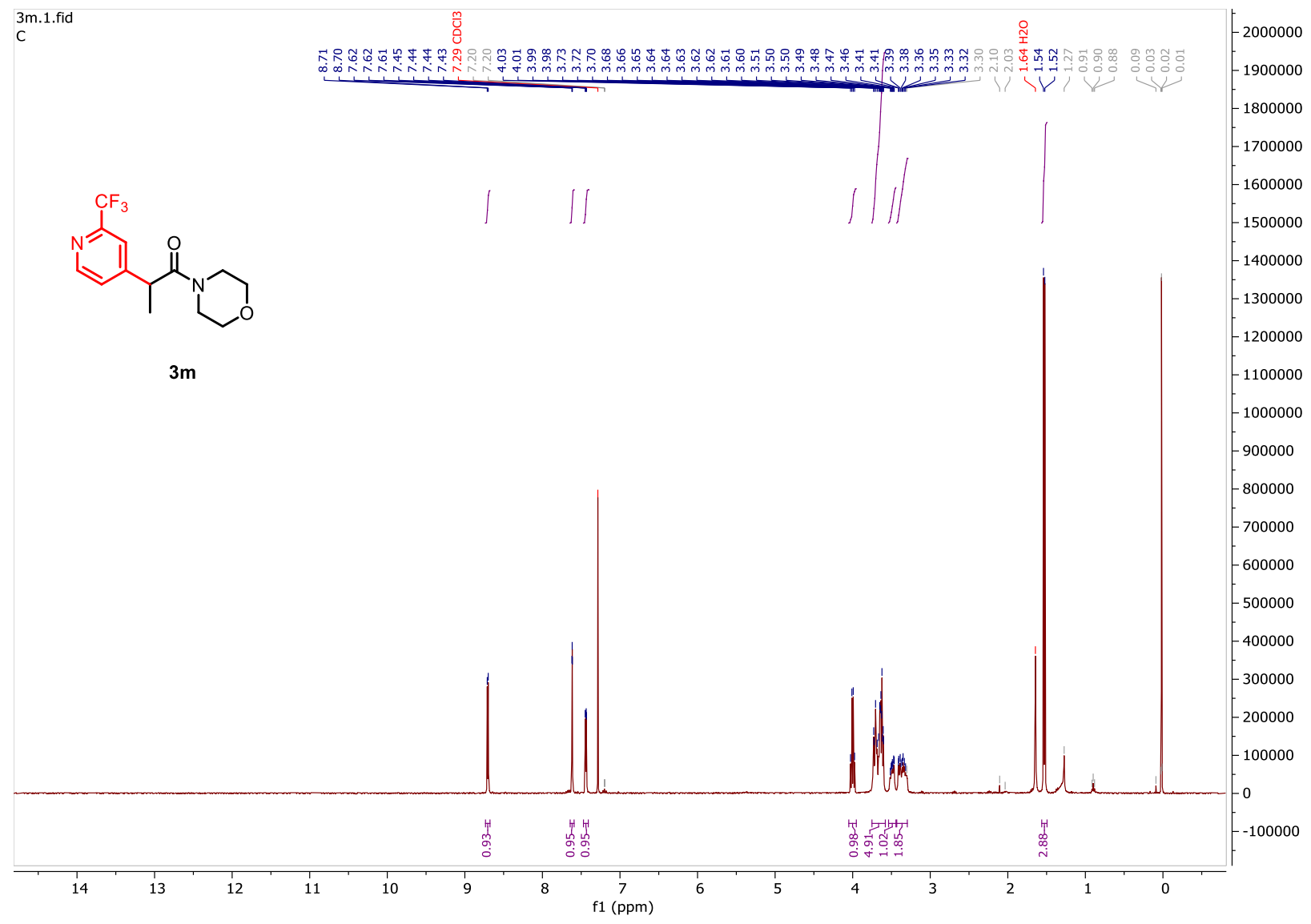




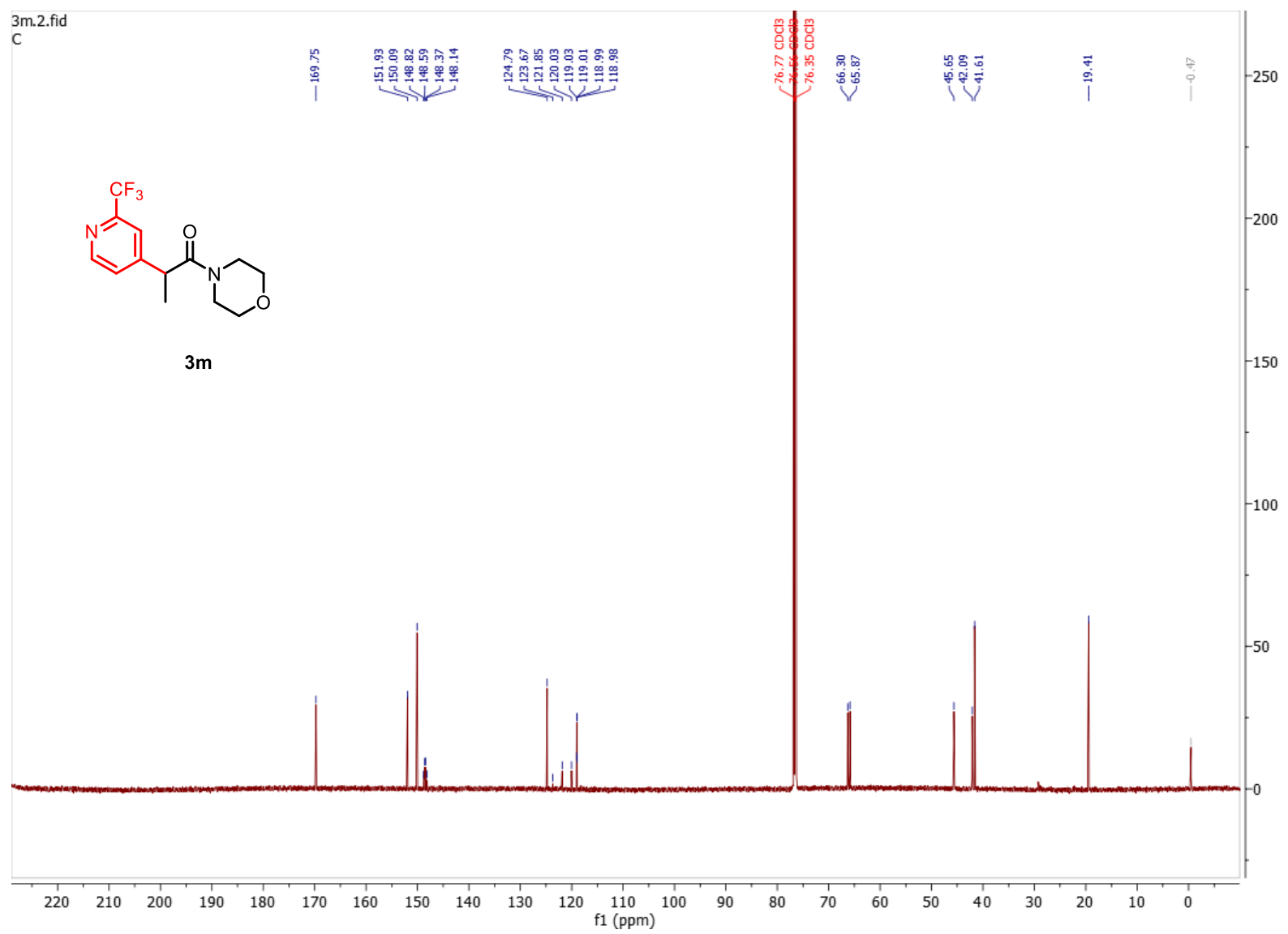




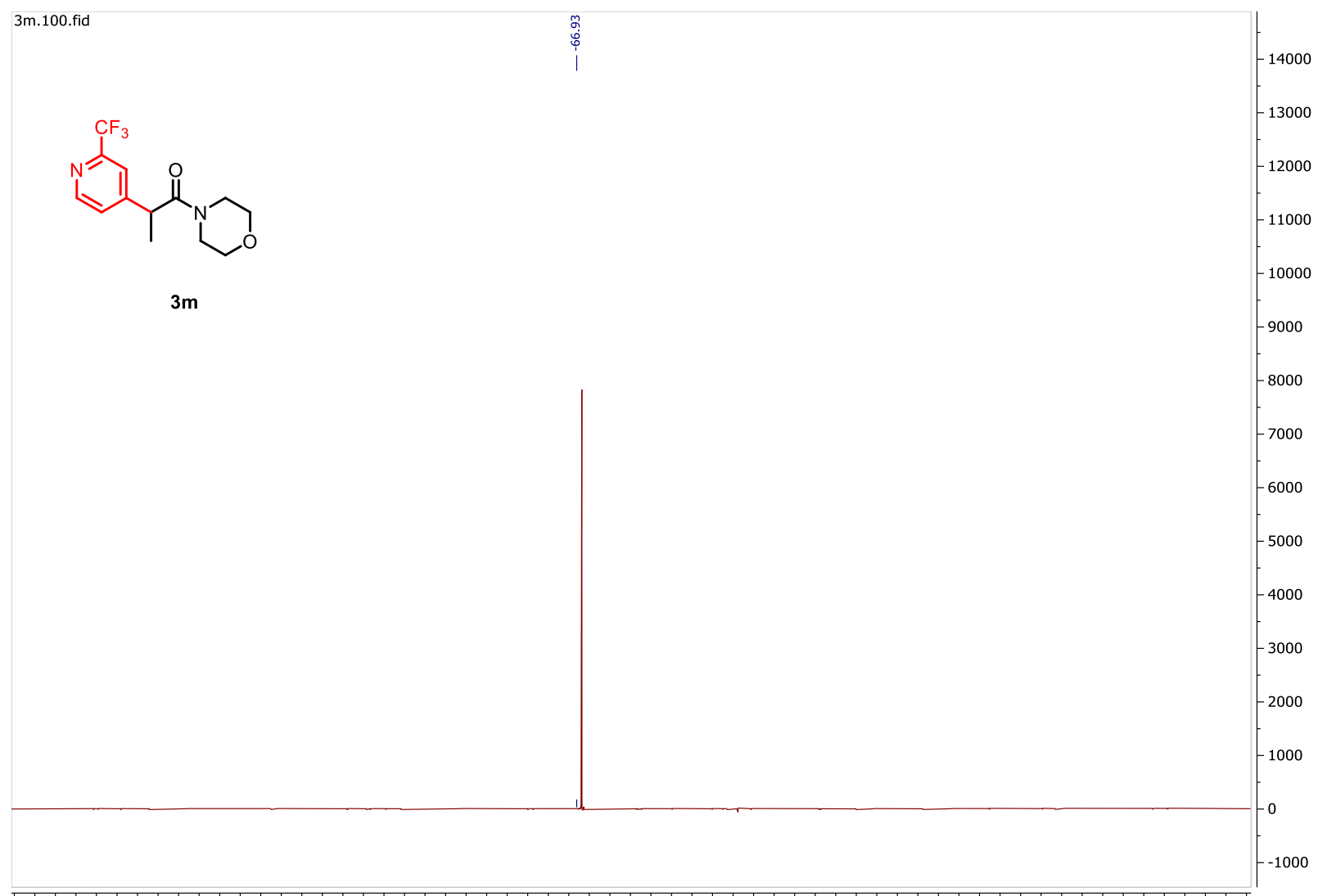

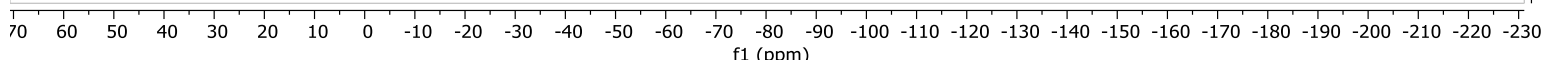




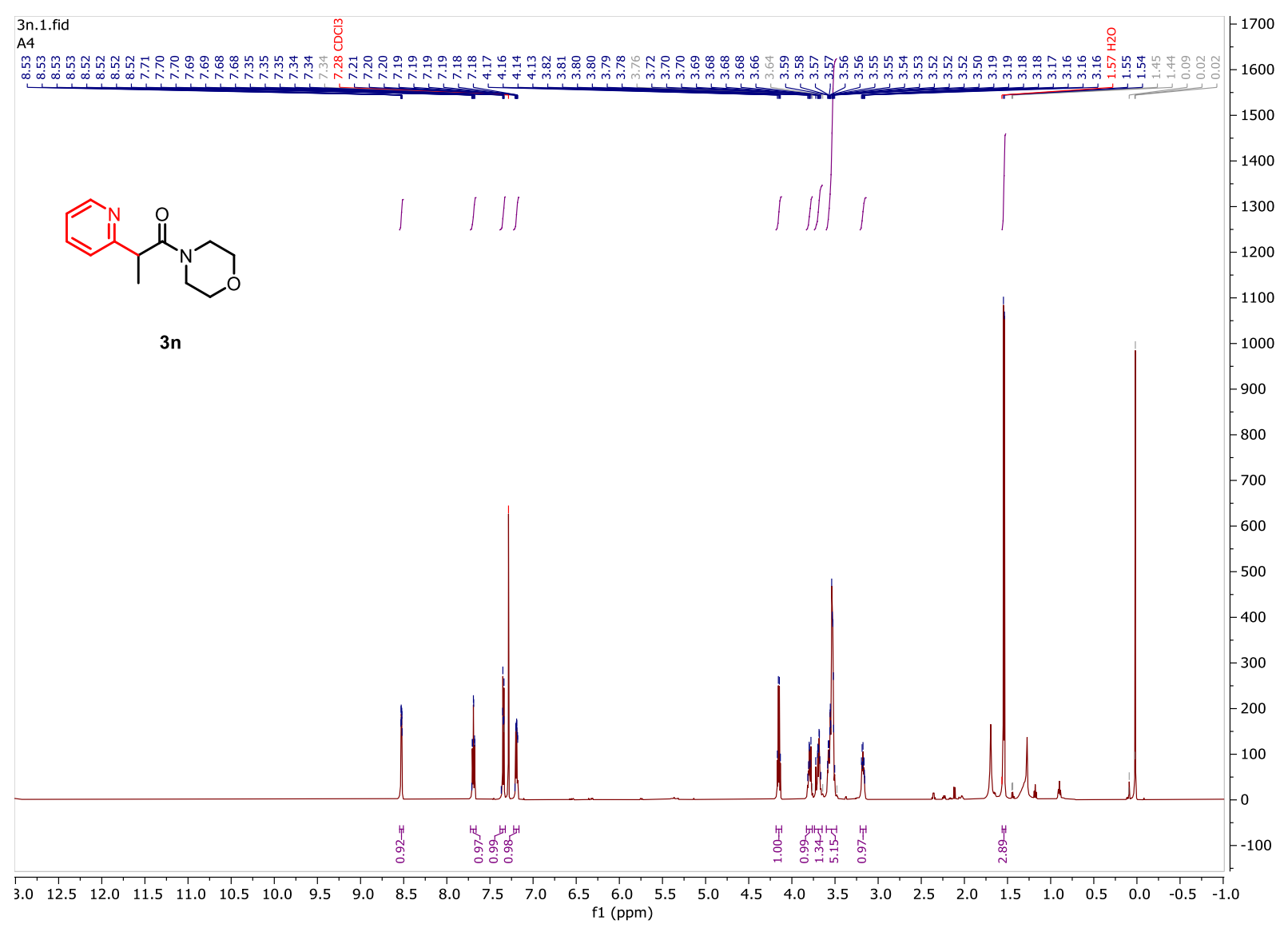




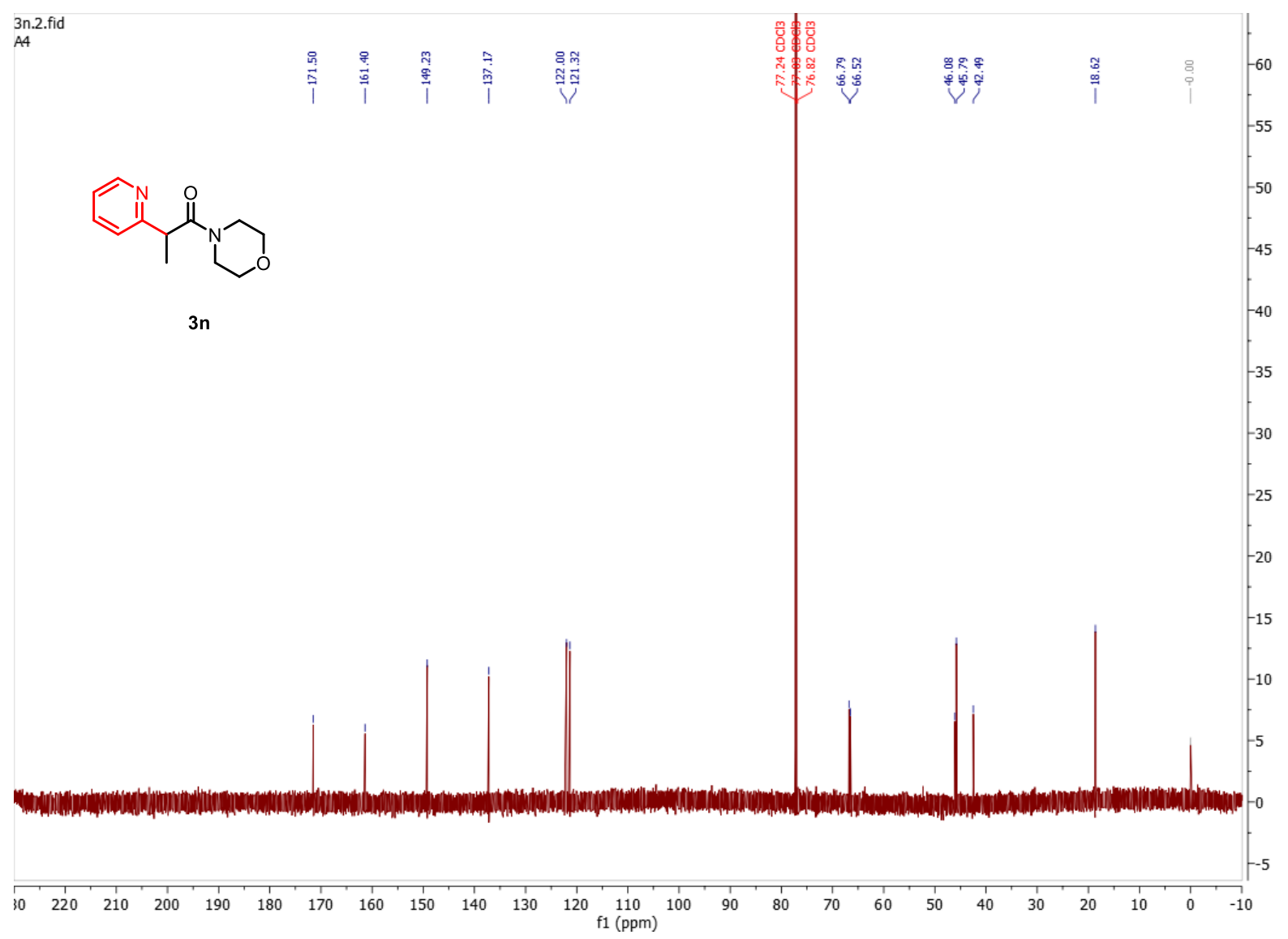




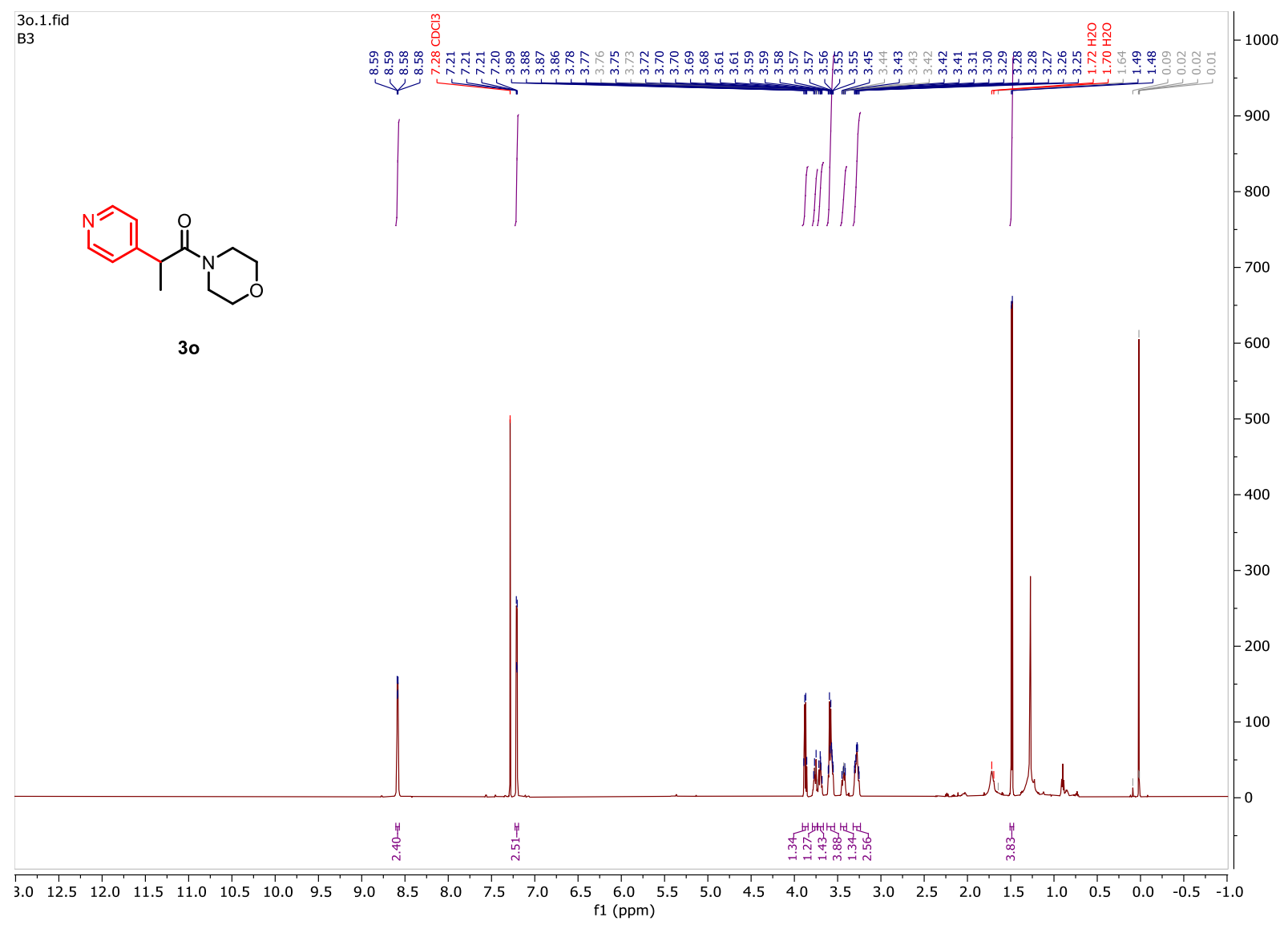




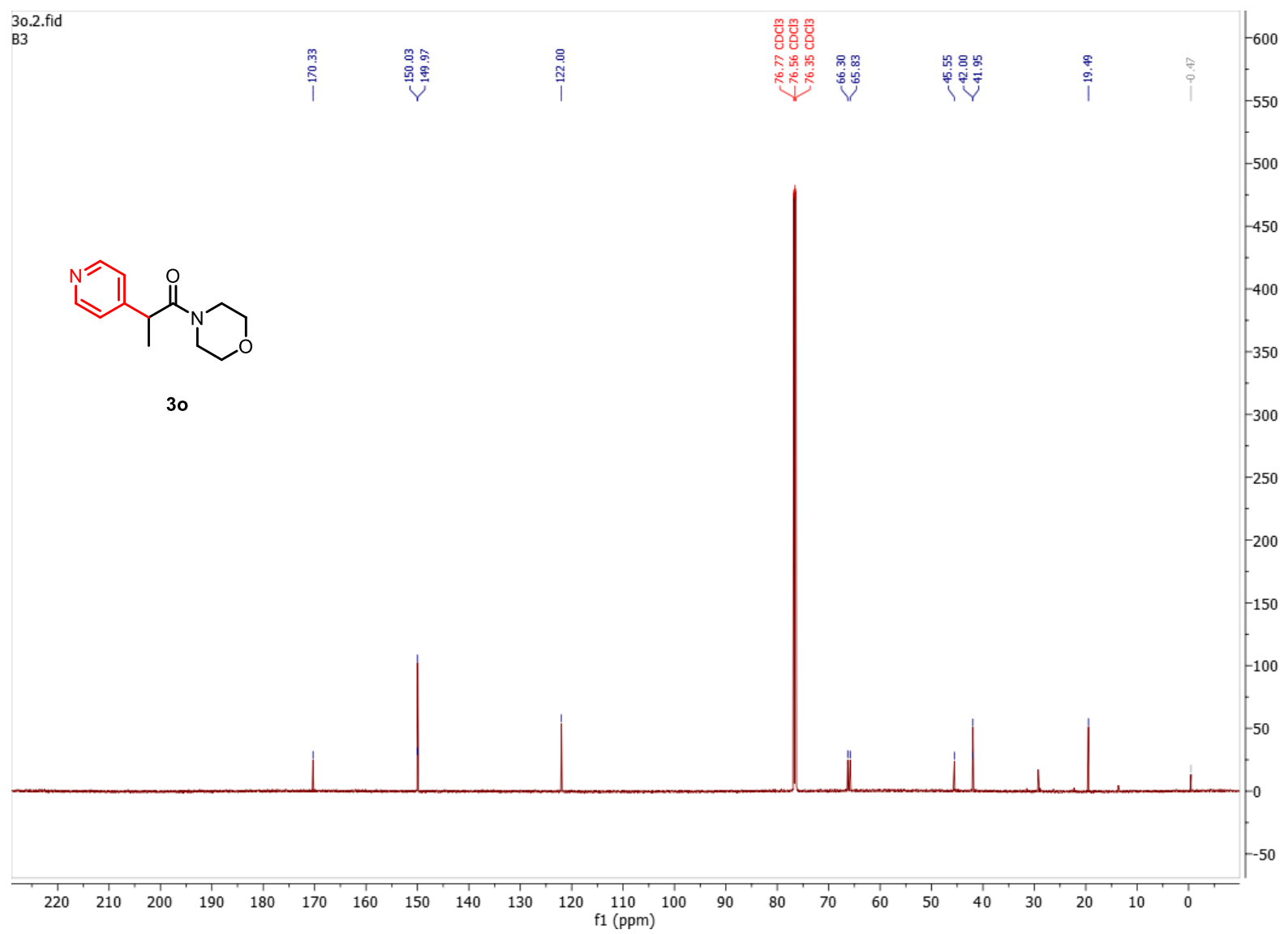









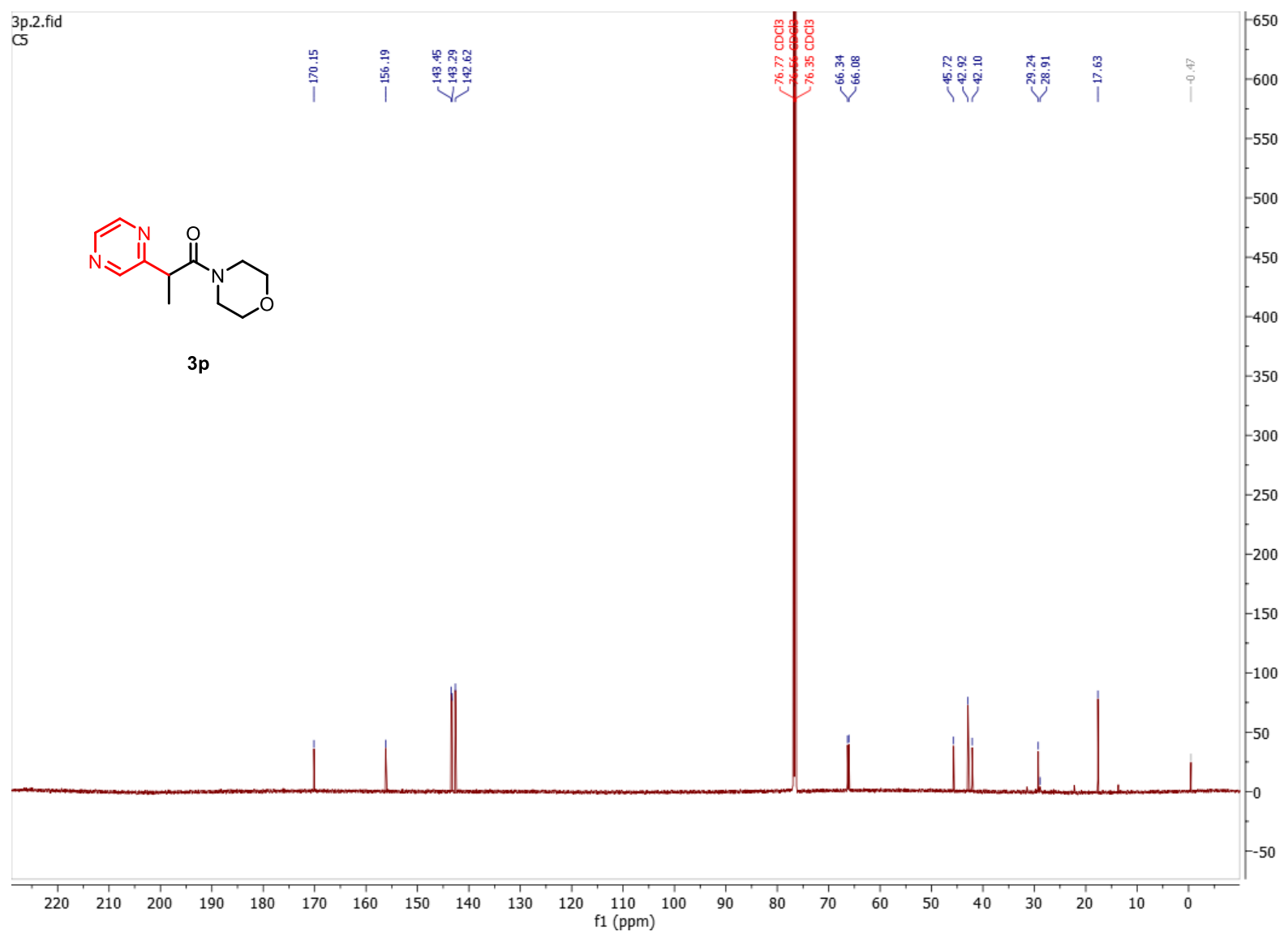




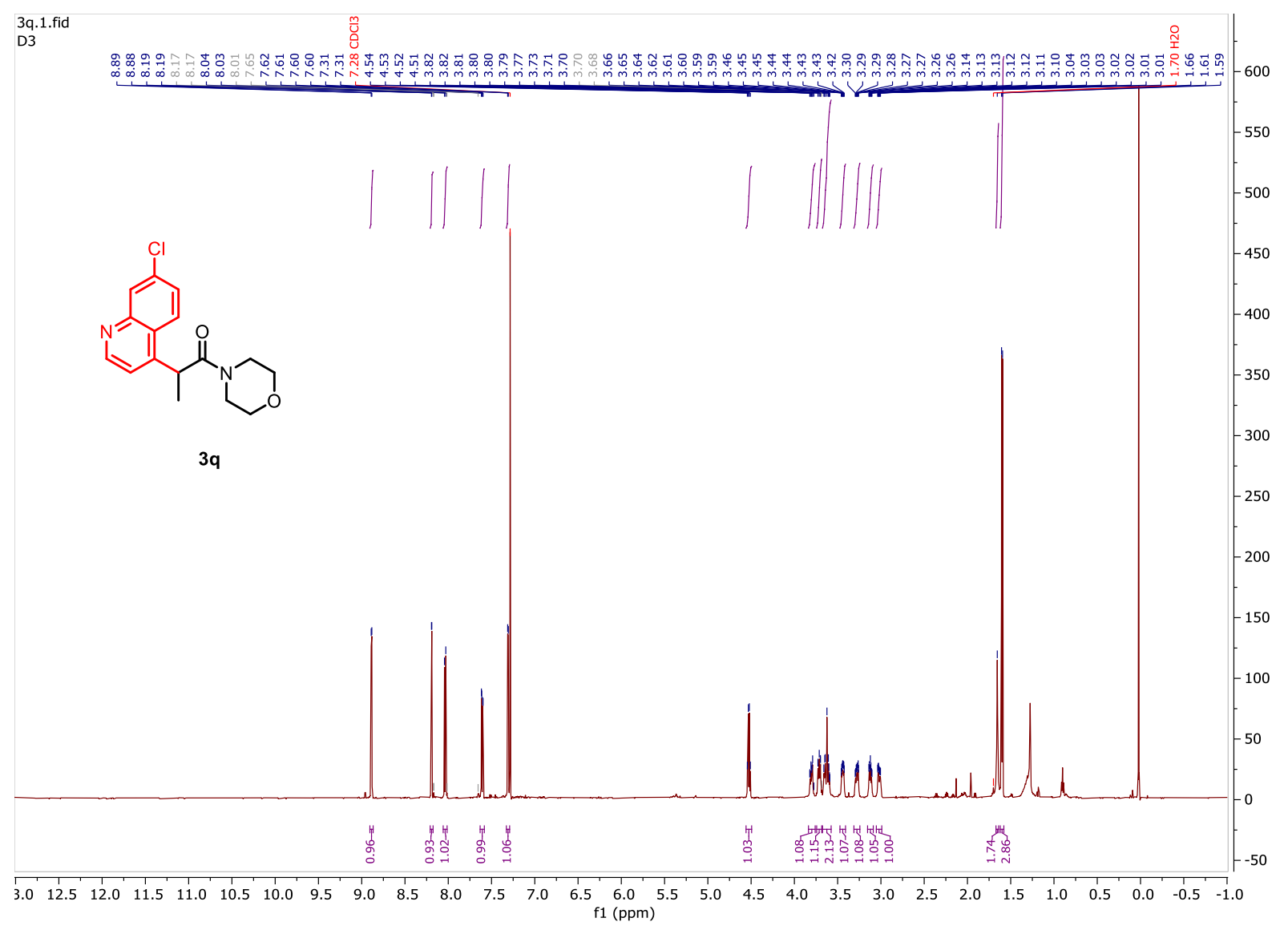




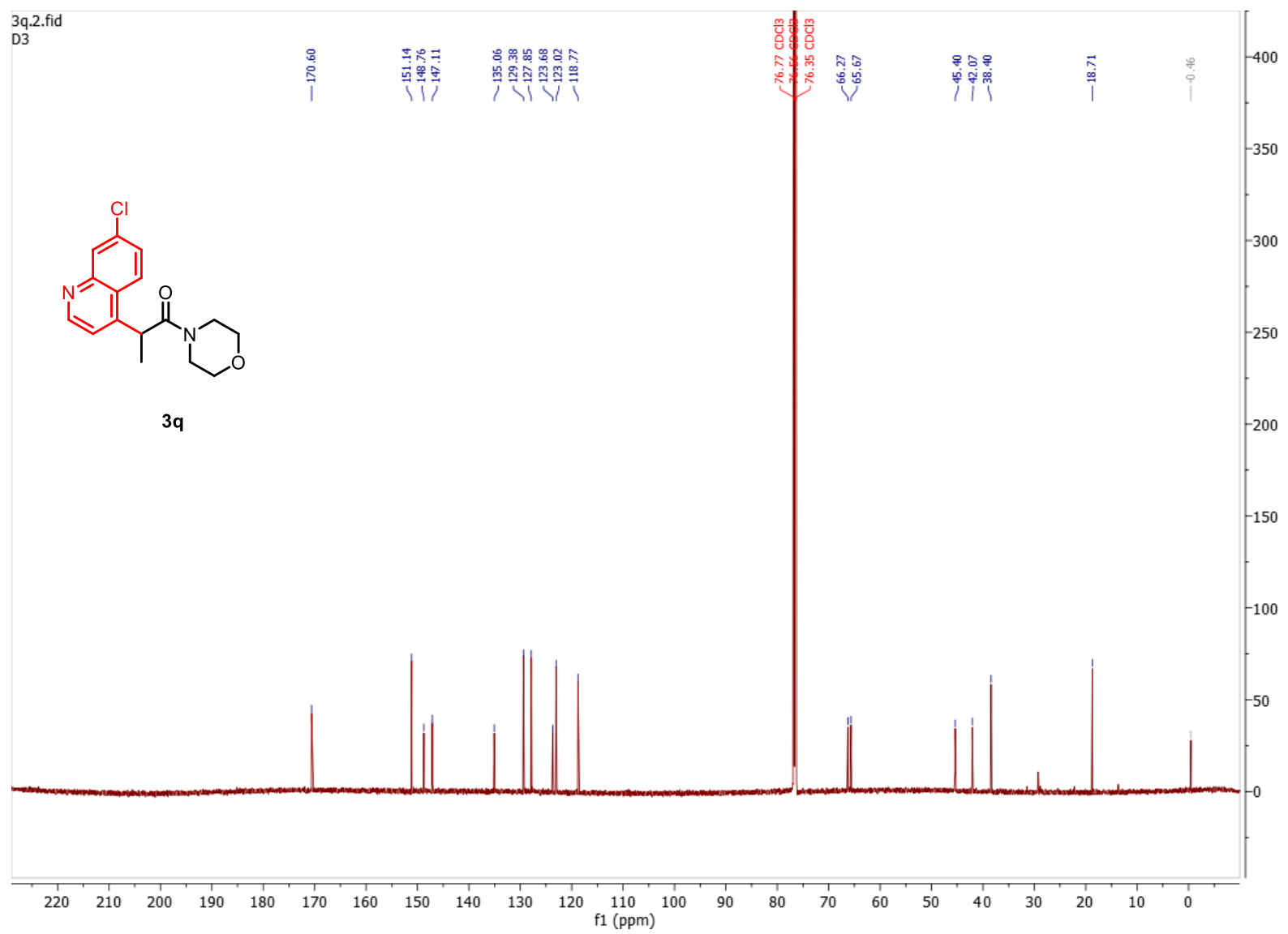




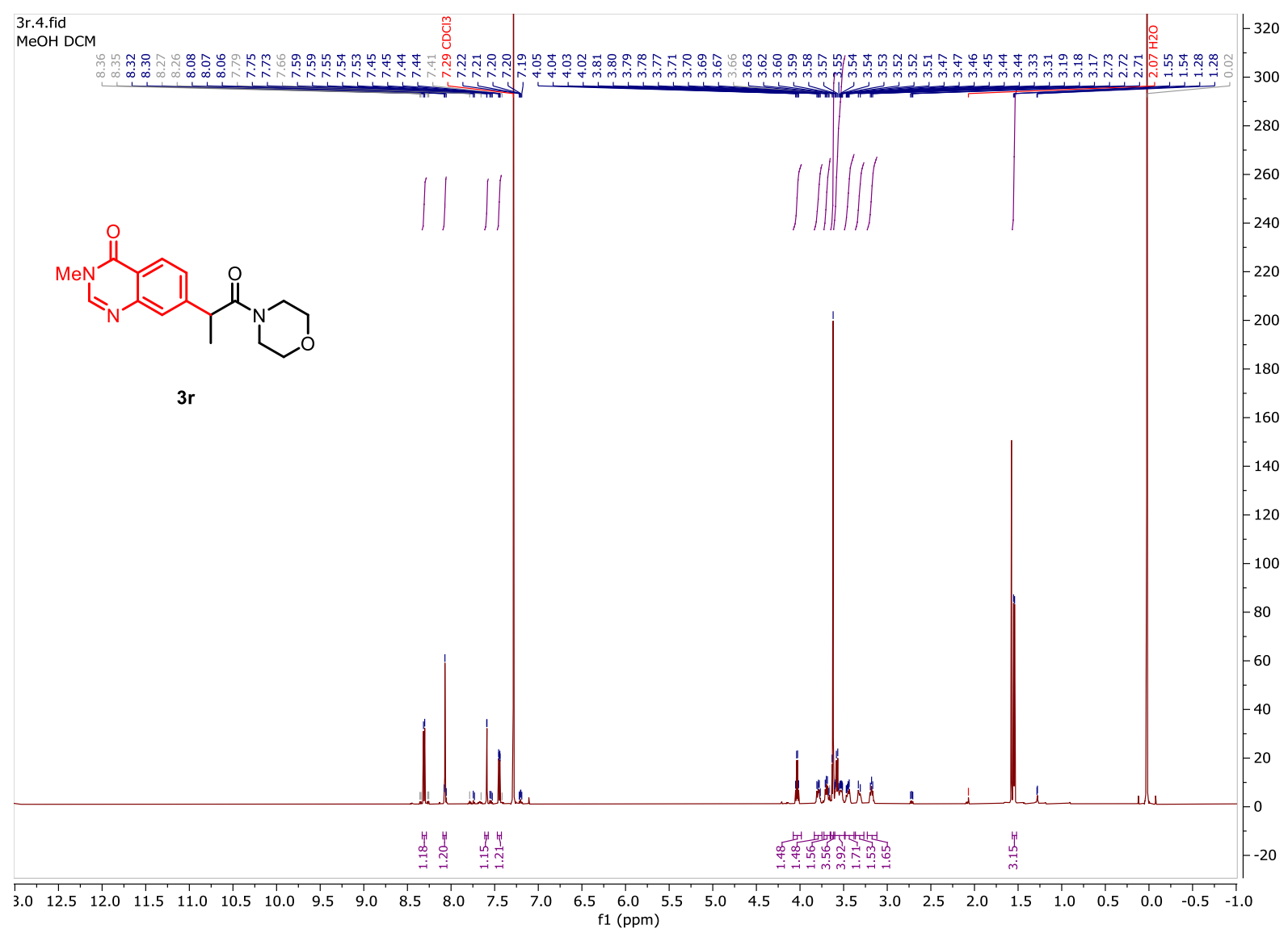




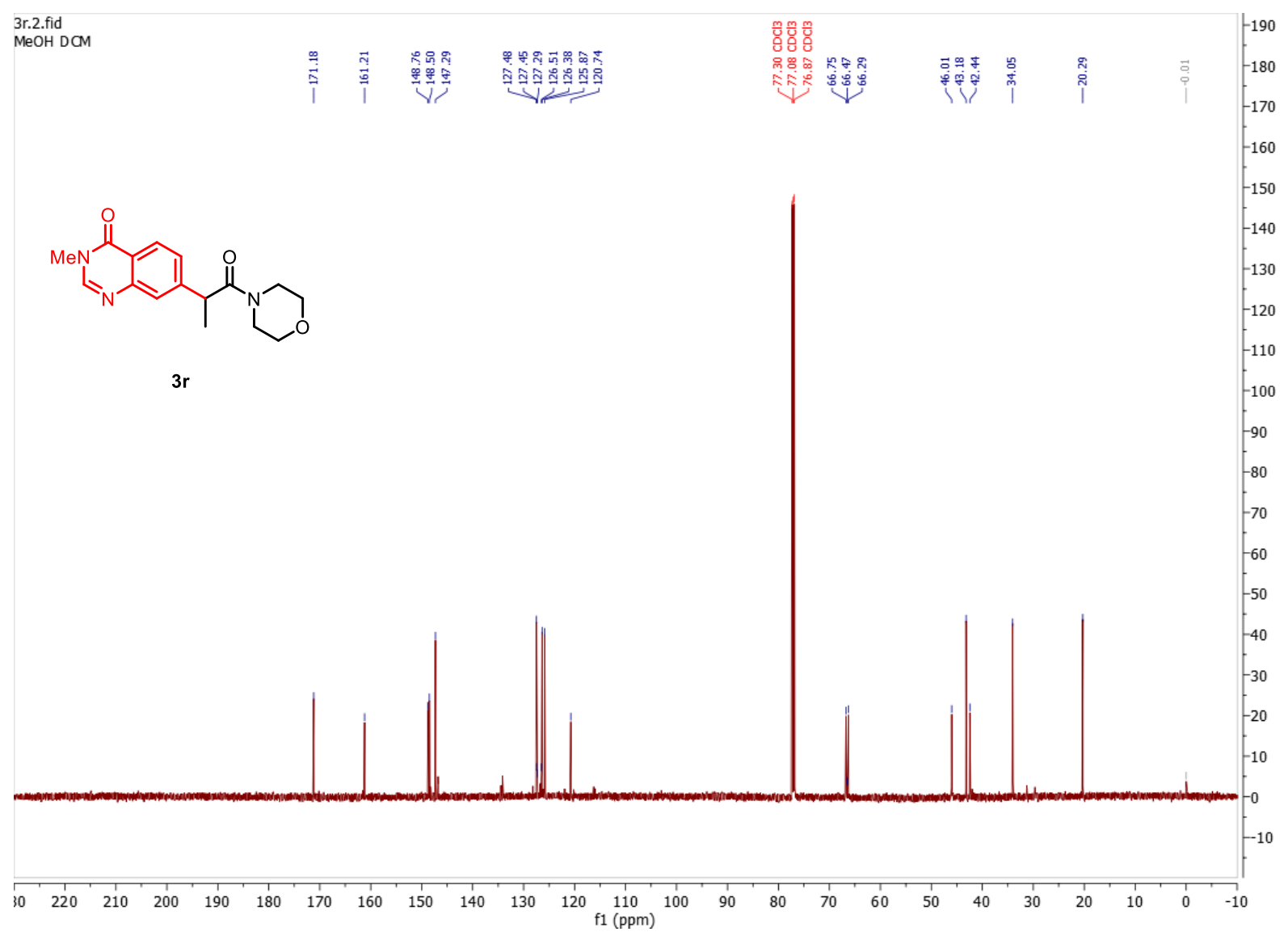




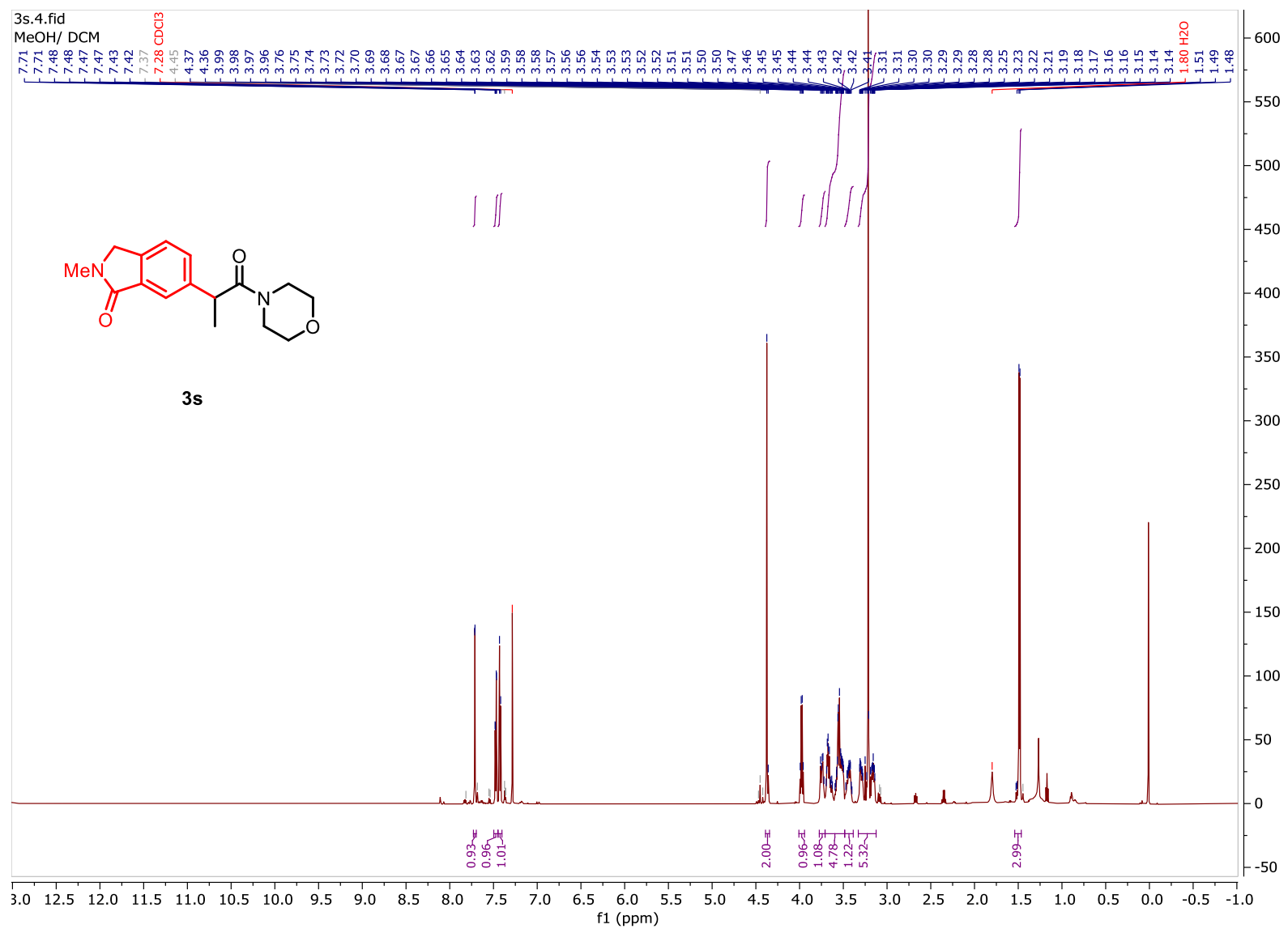

S90 


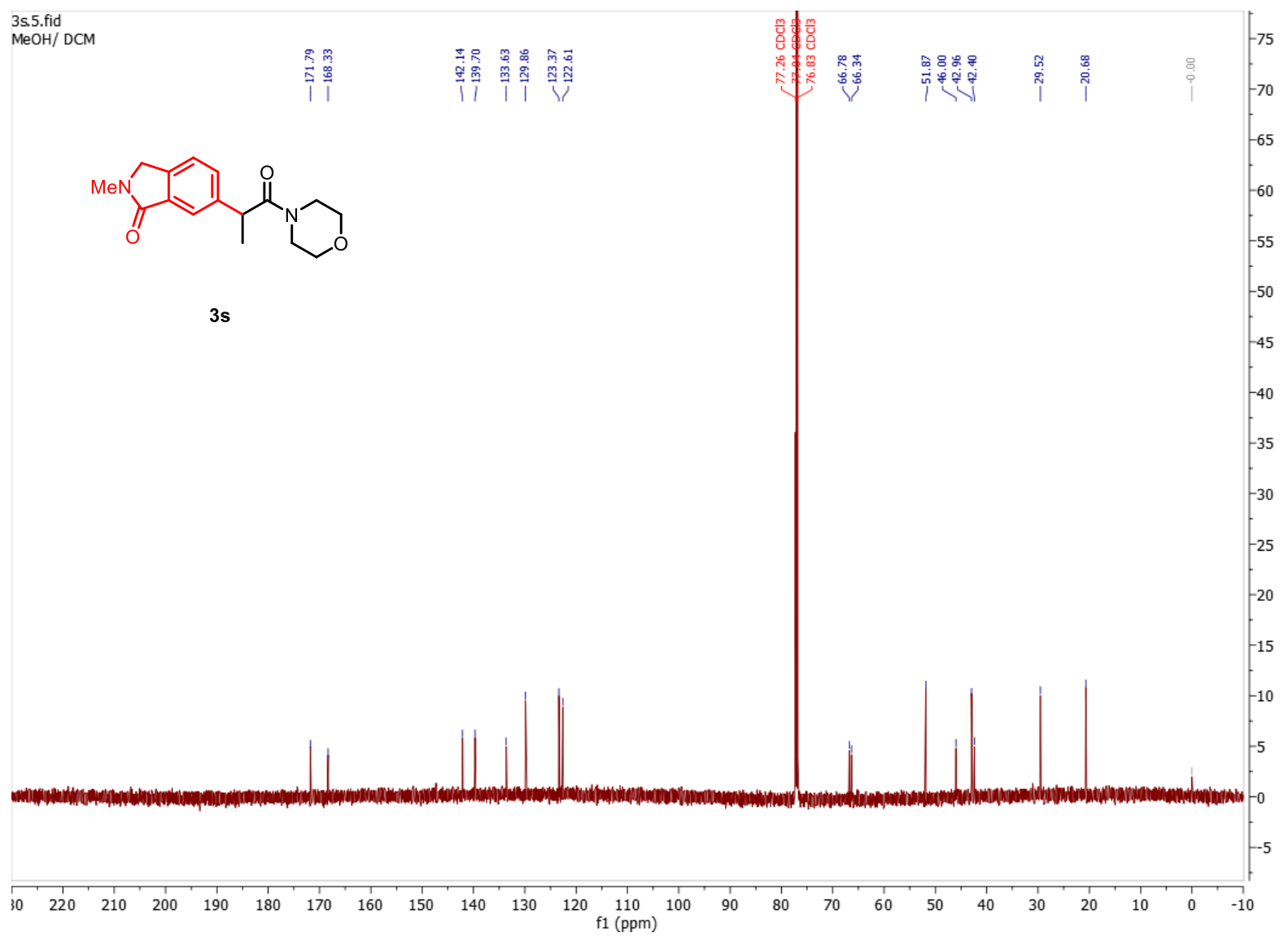




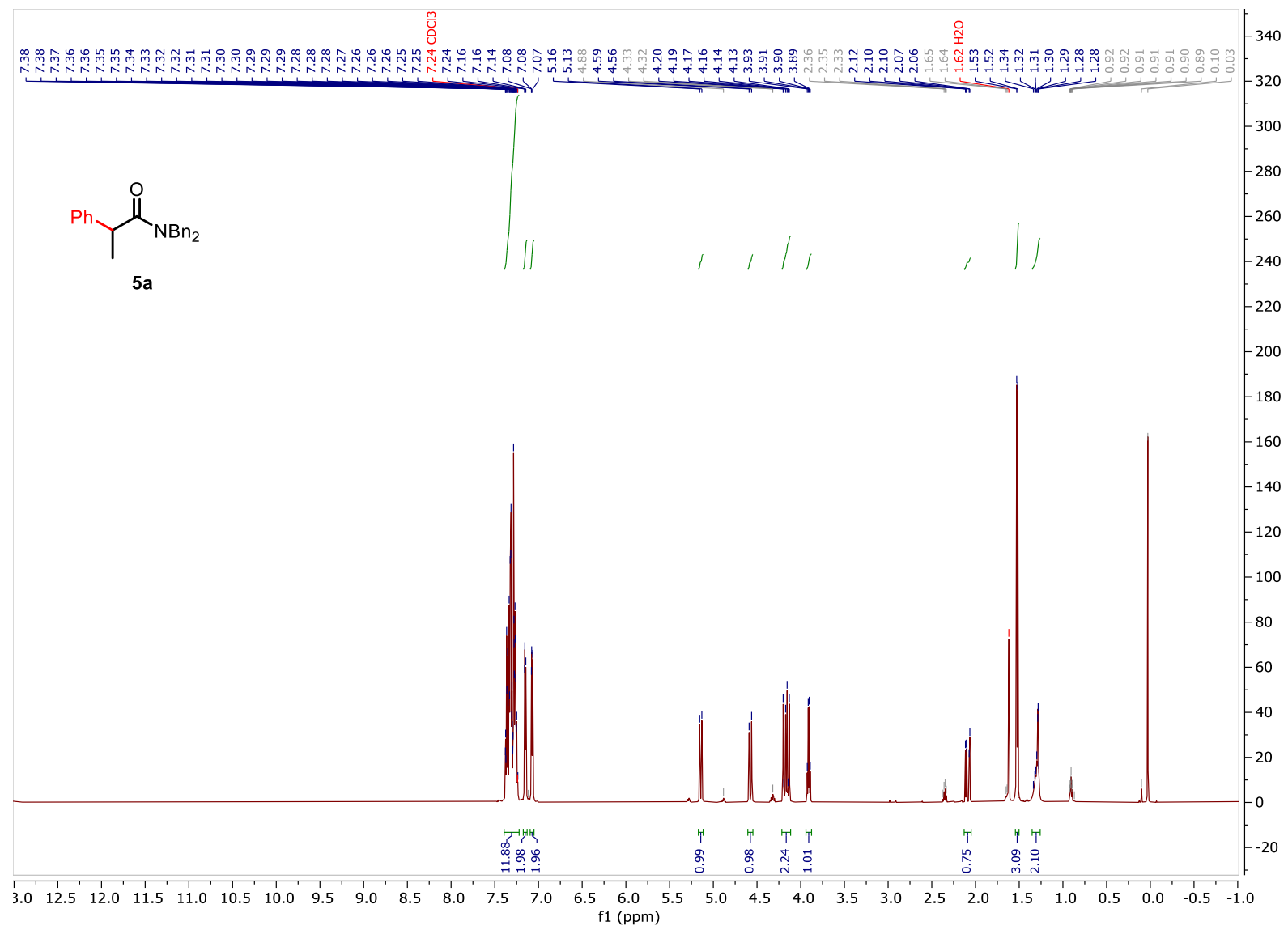




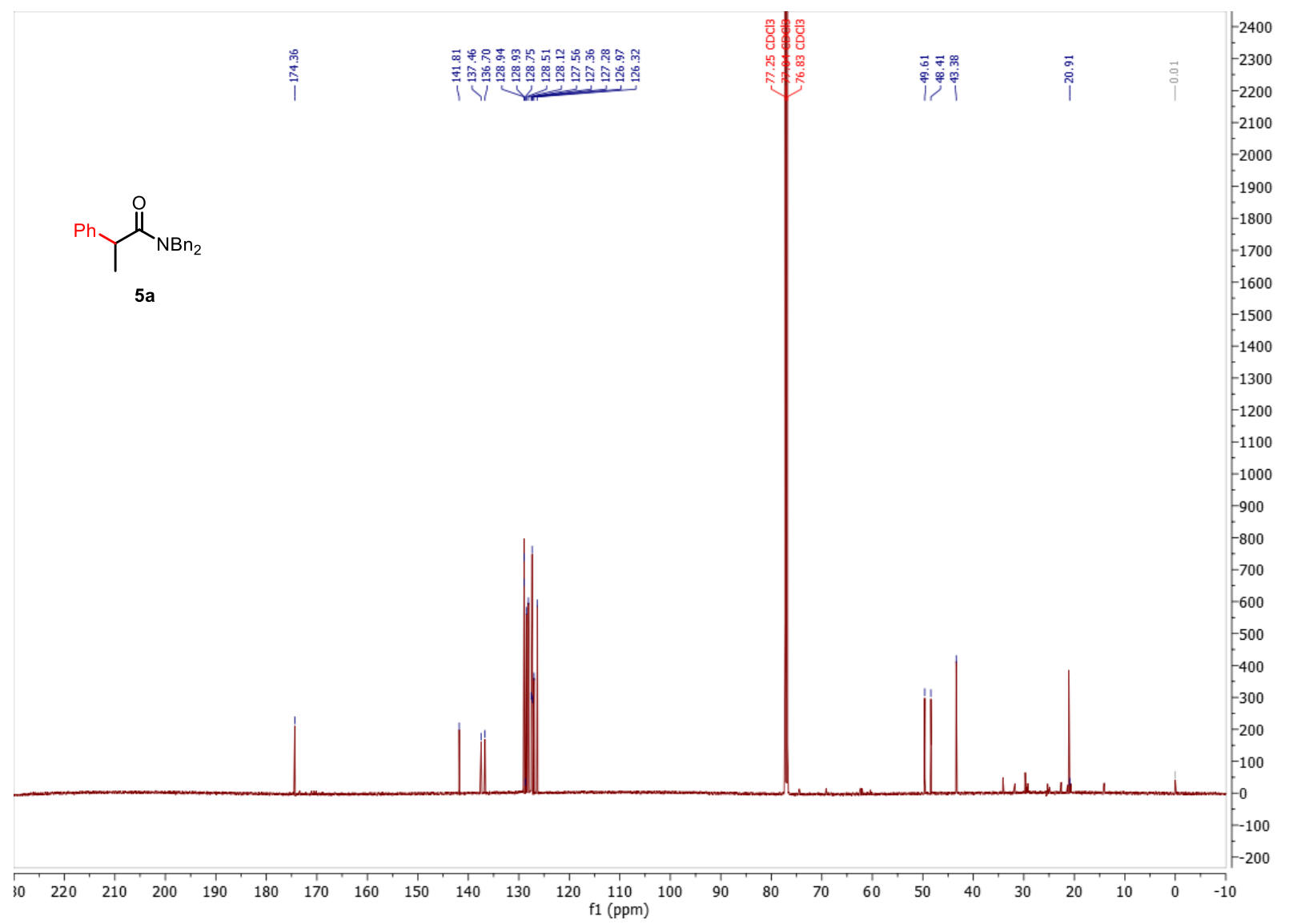




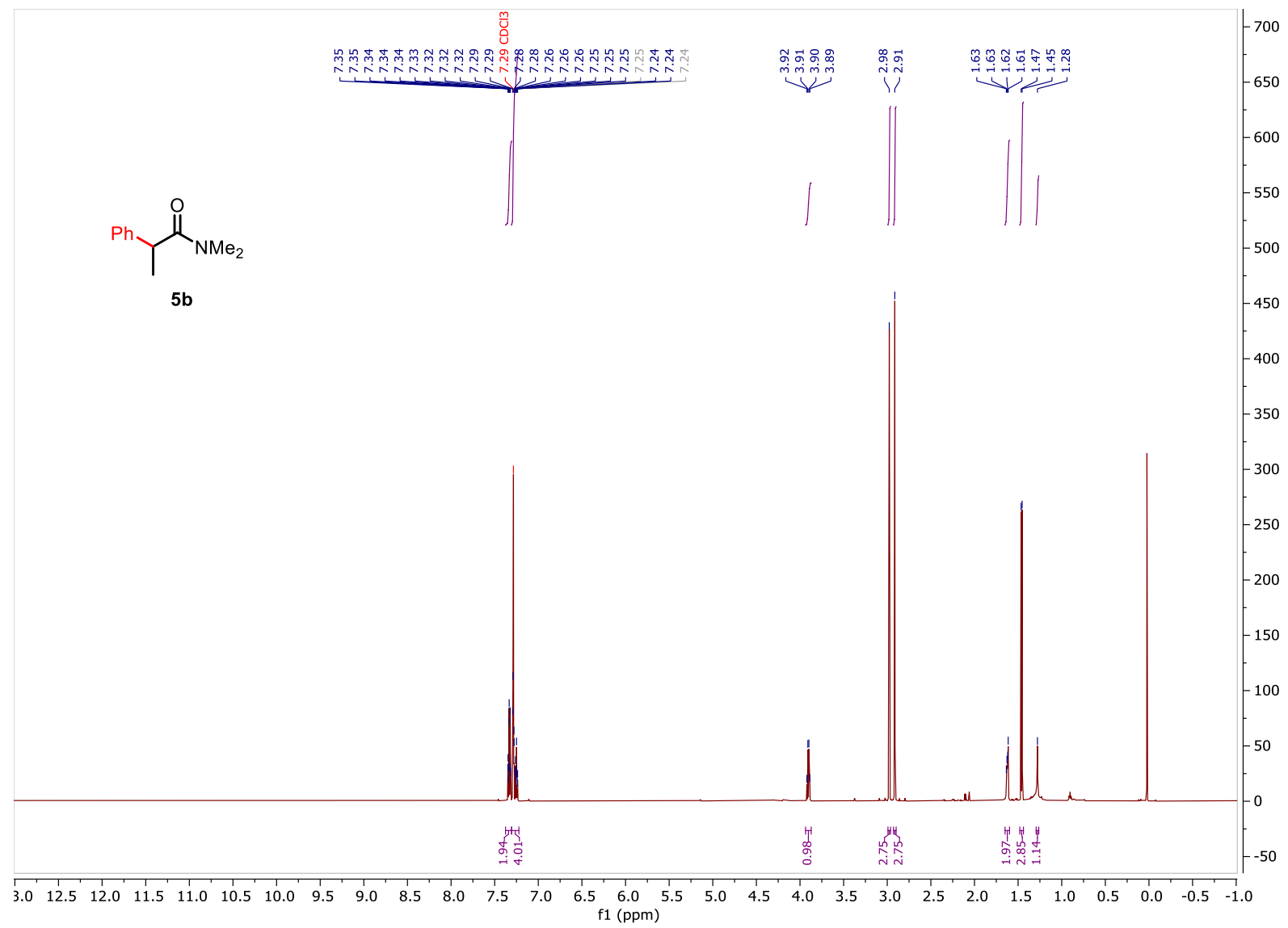




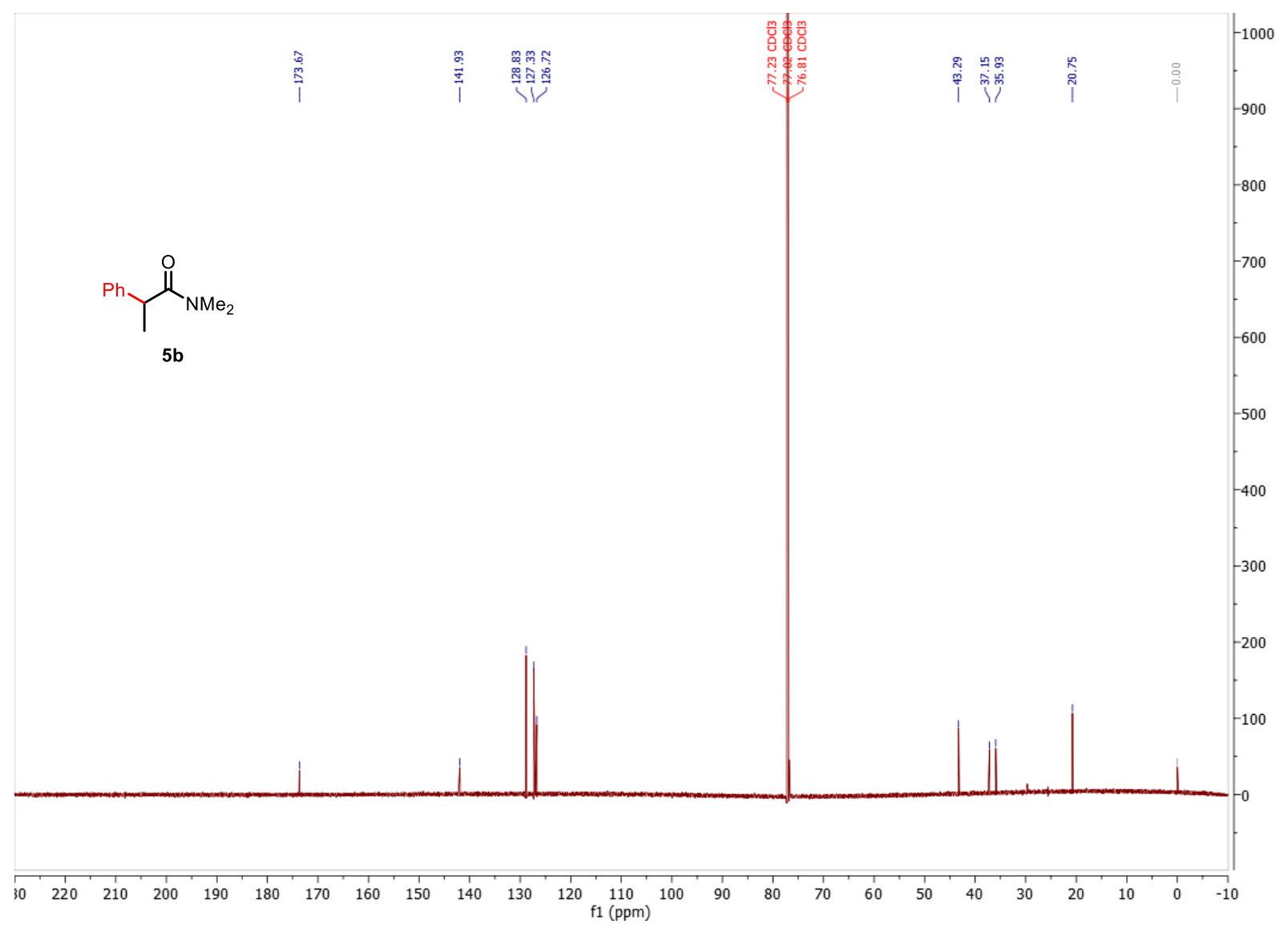




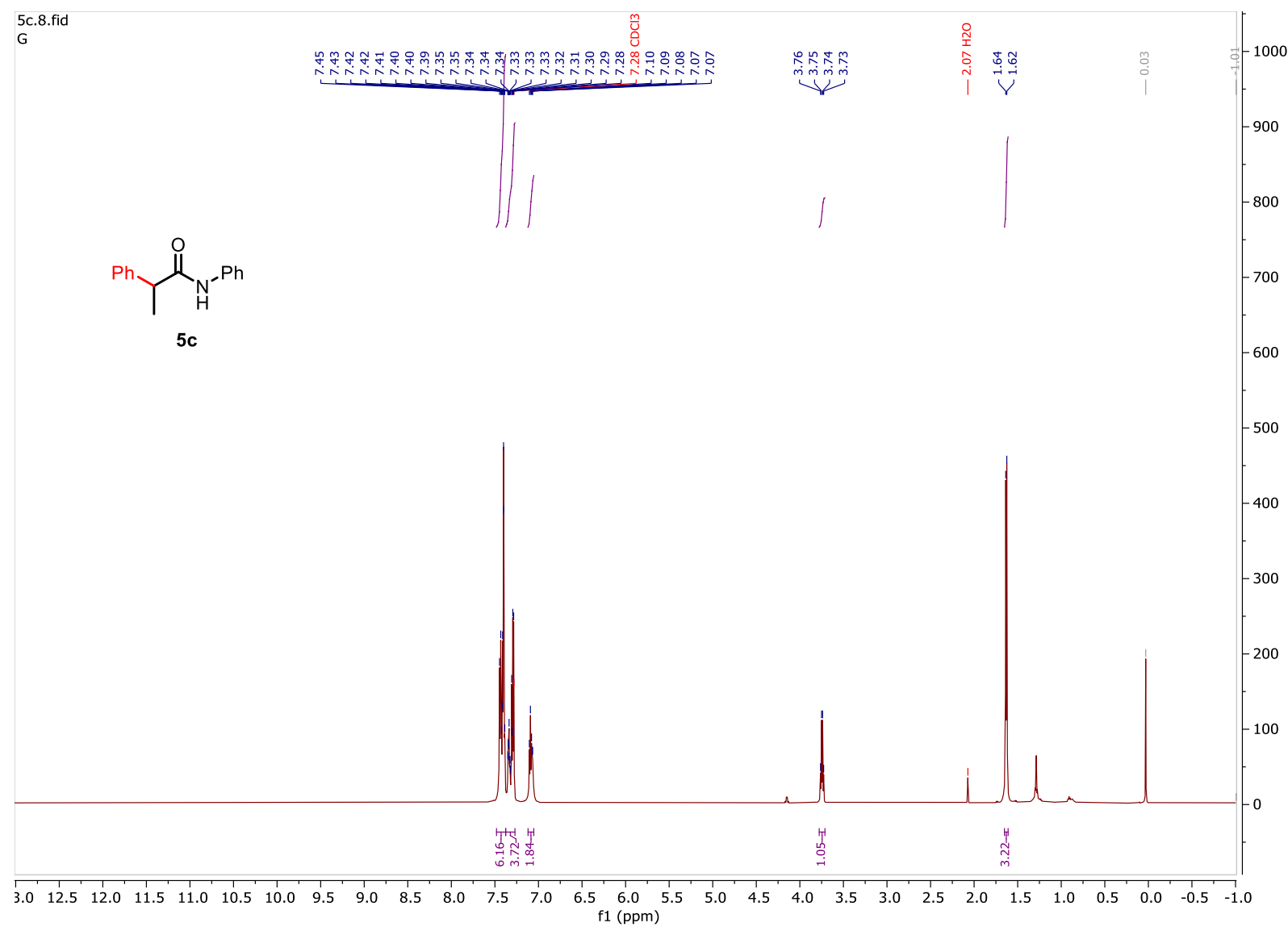




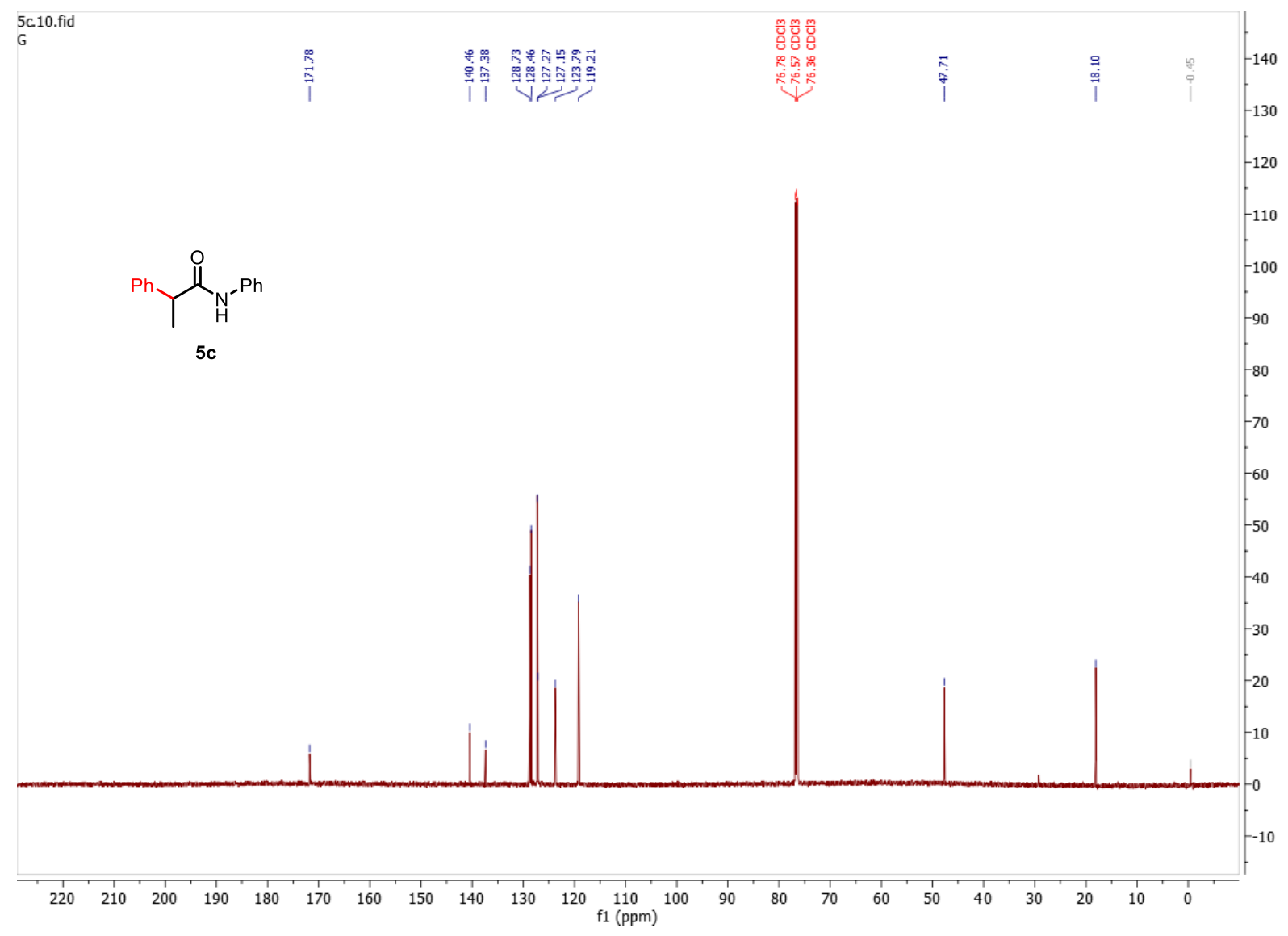




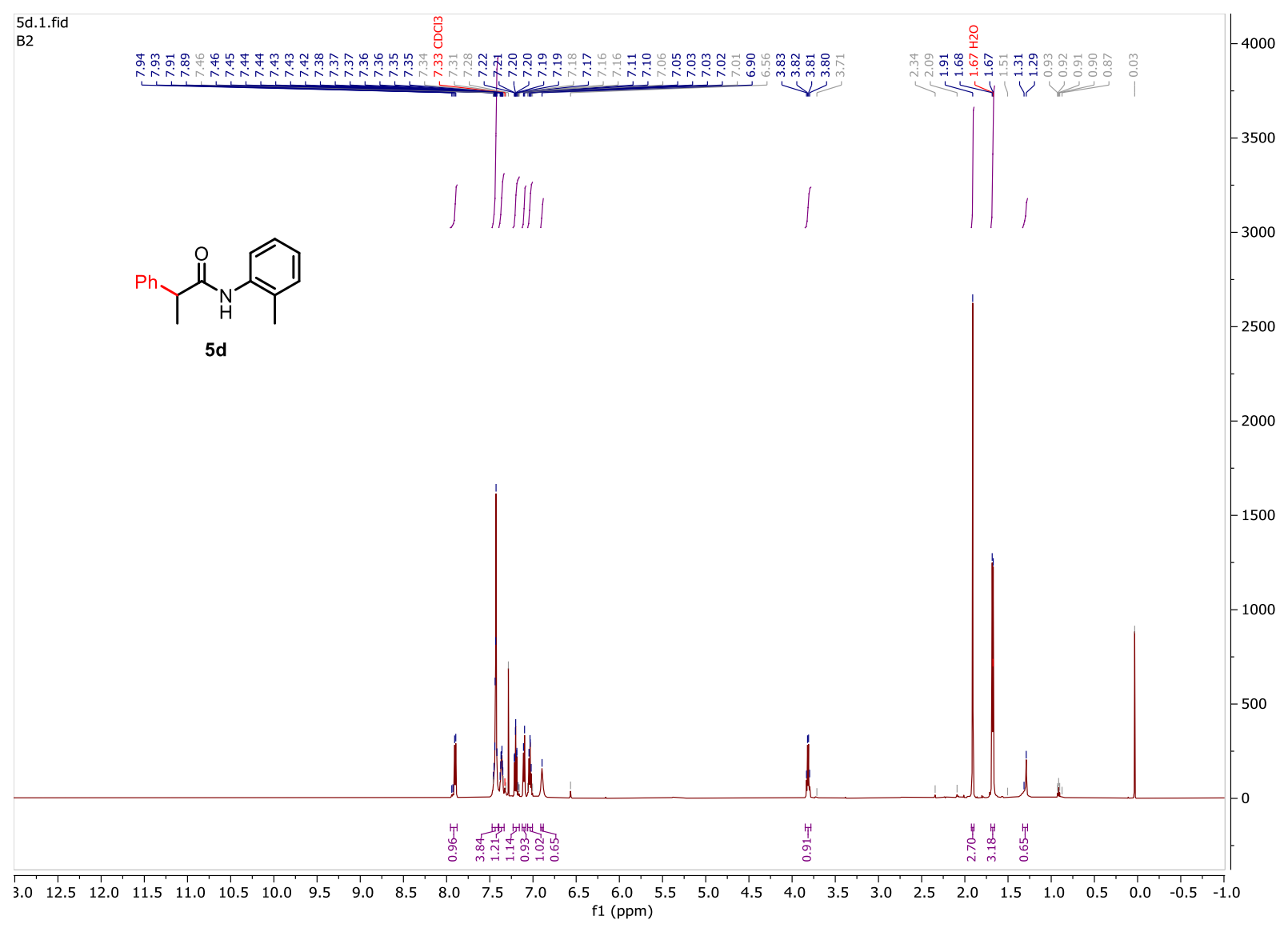




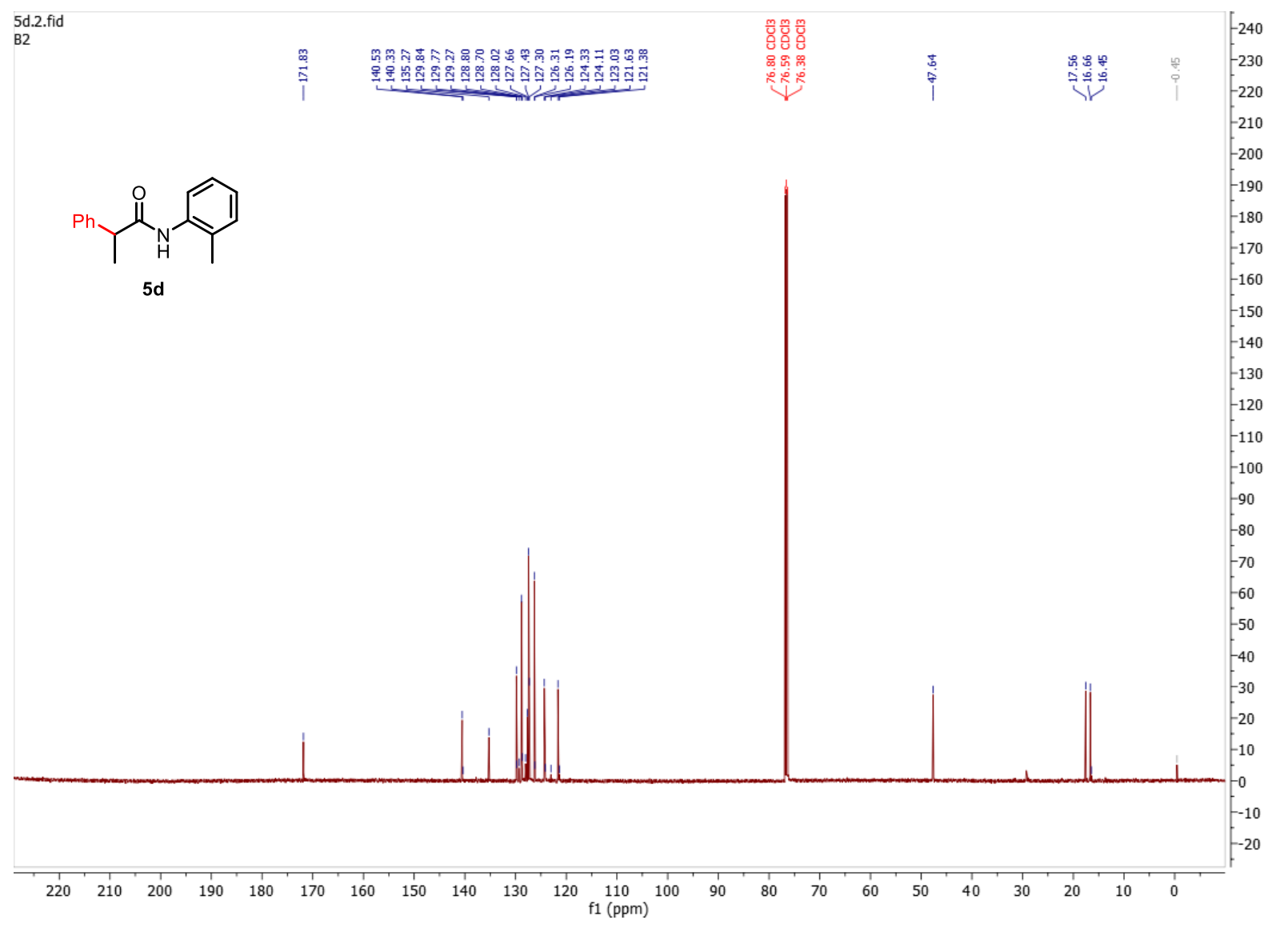




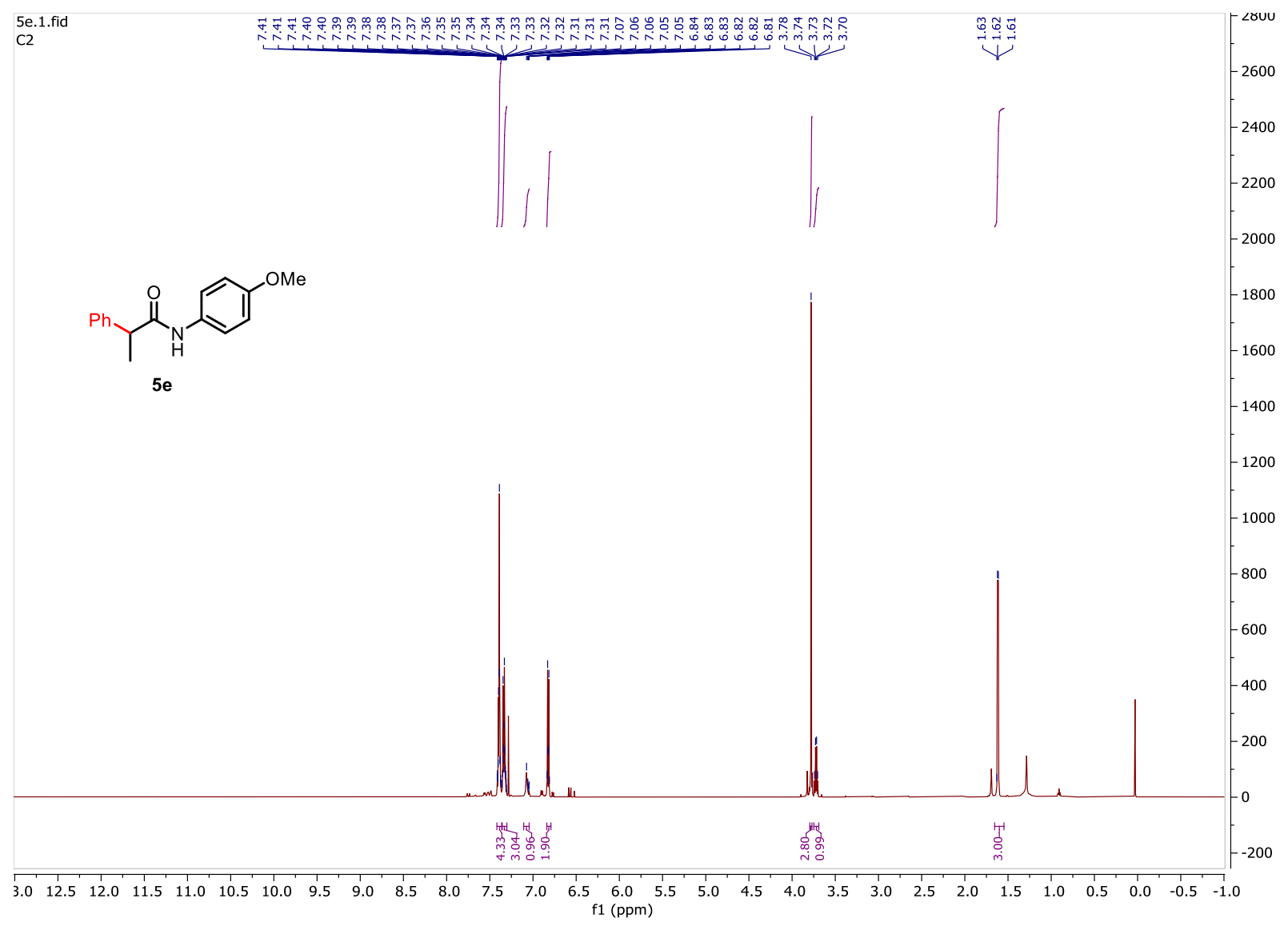




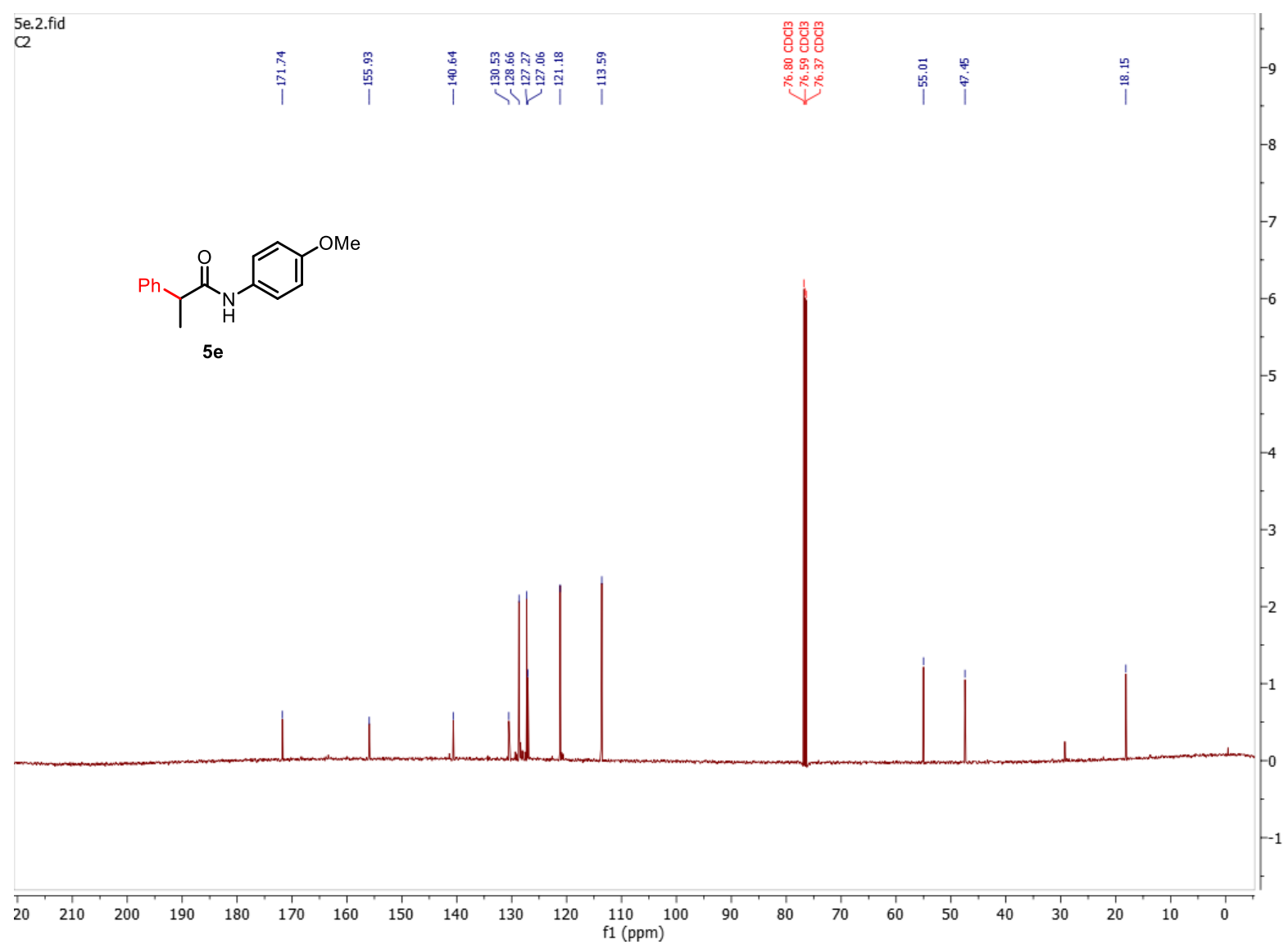




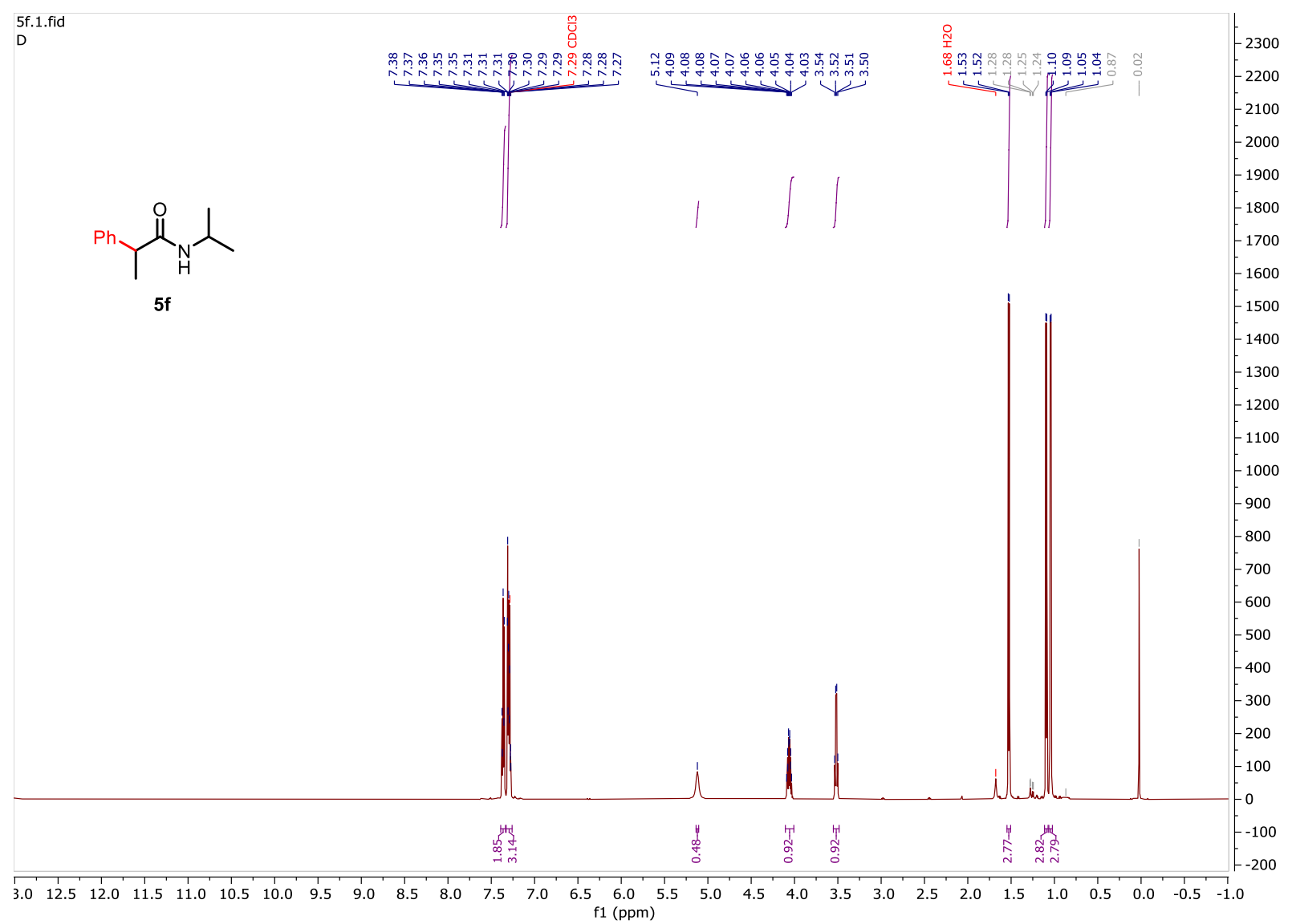




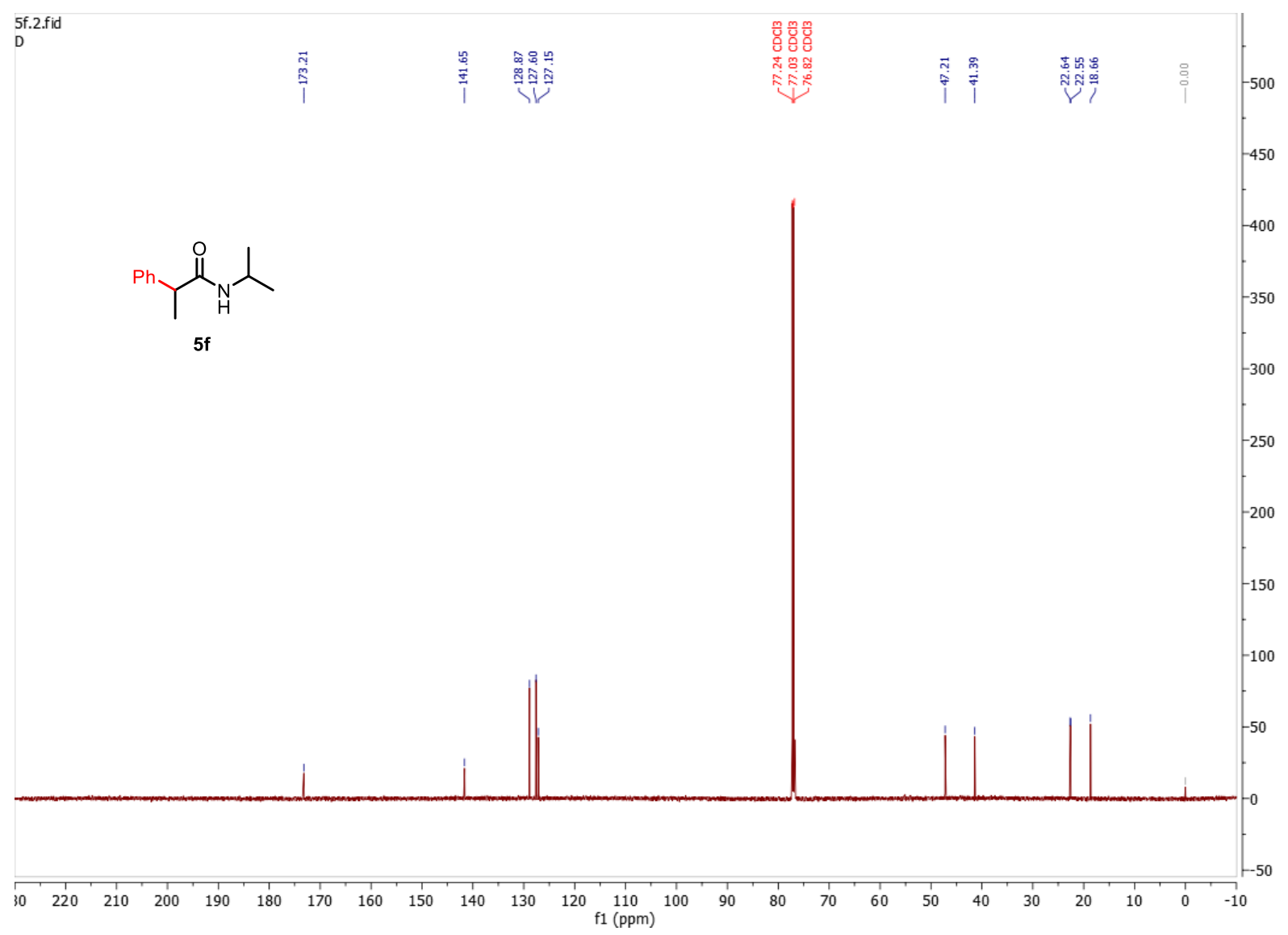




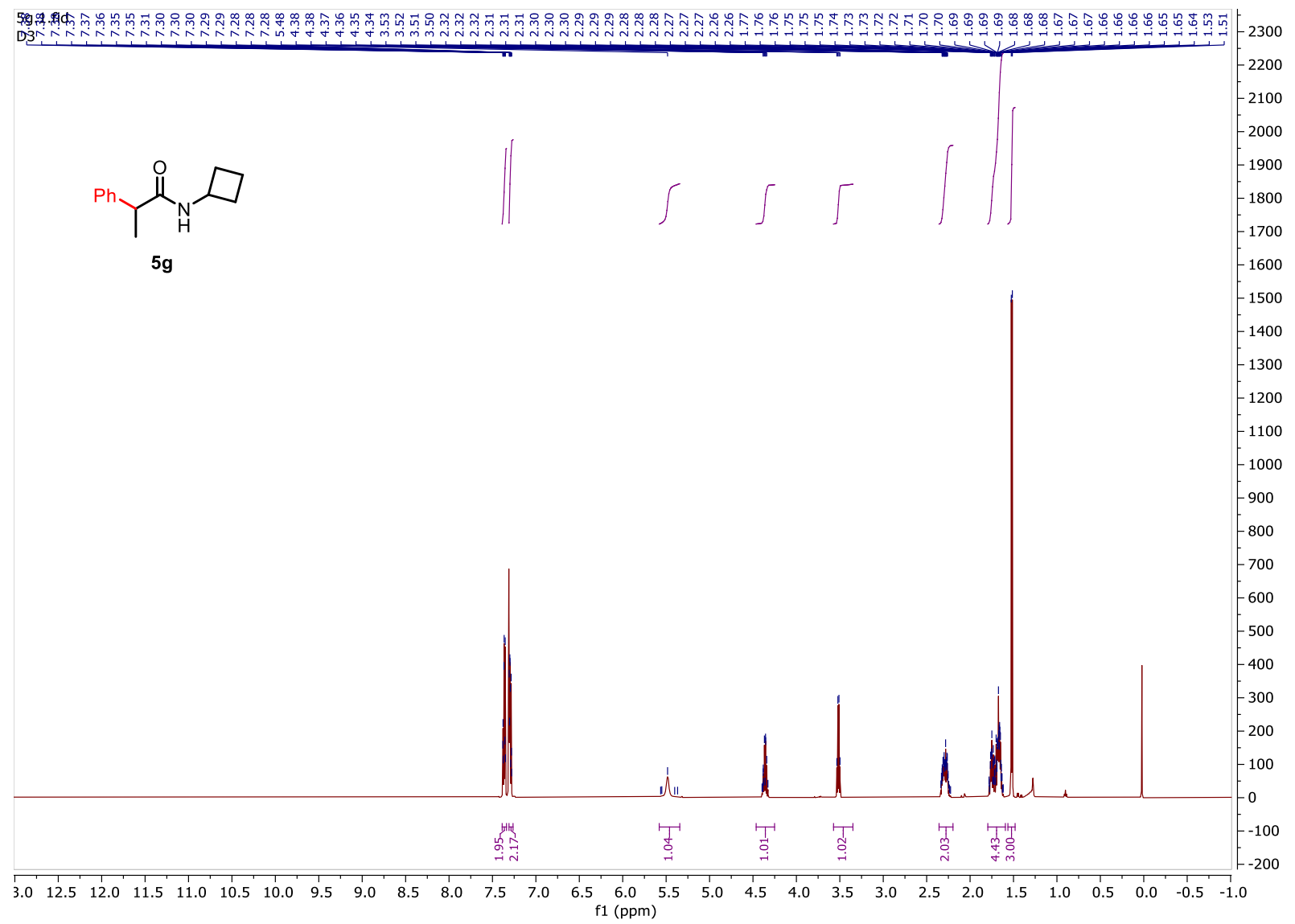




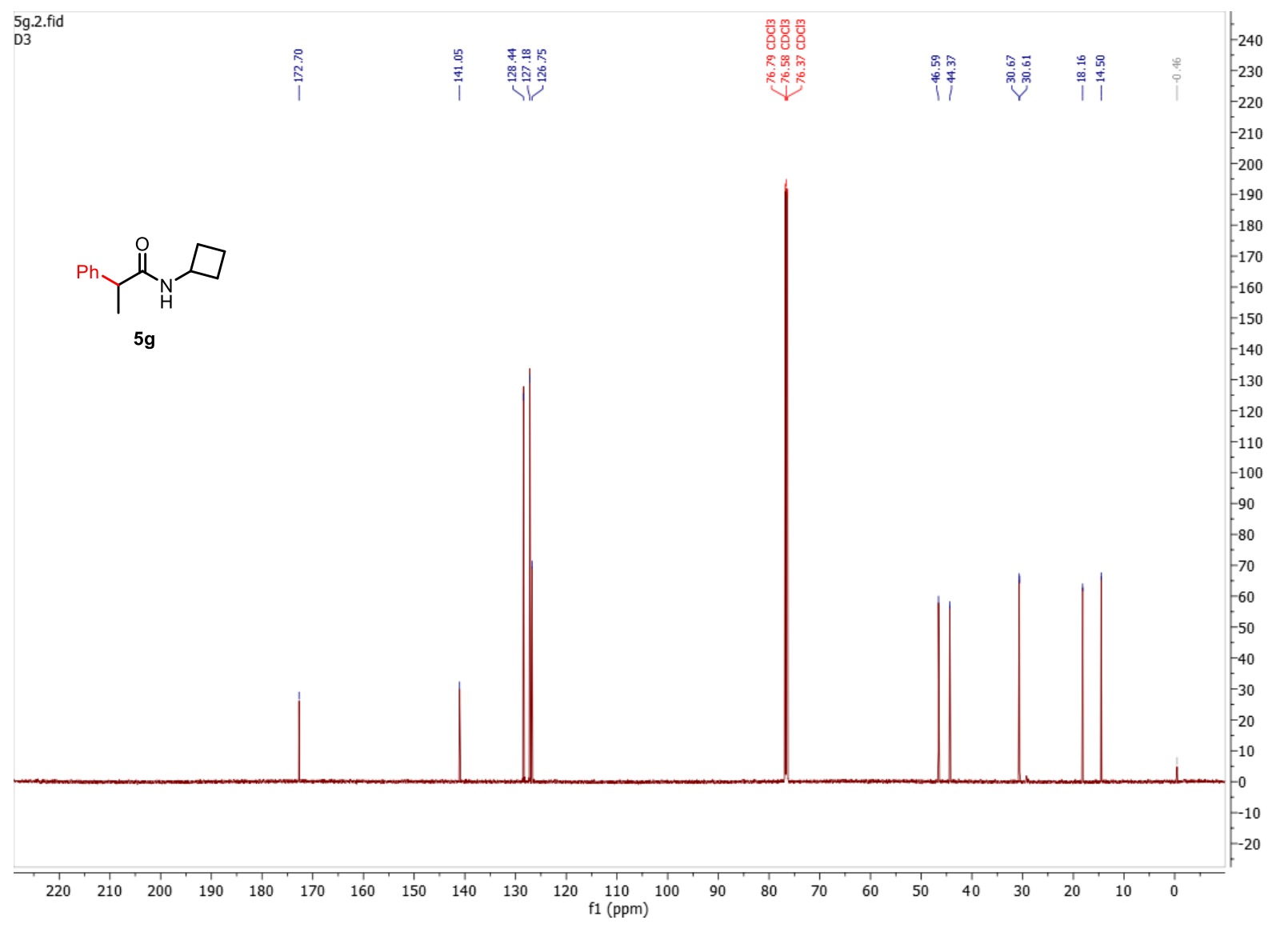




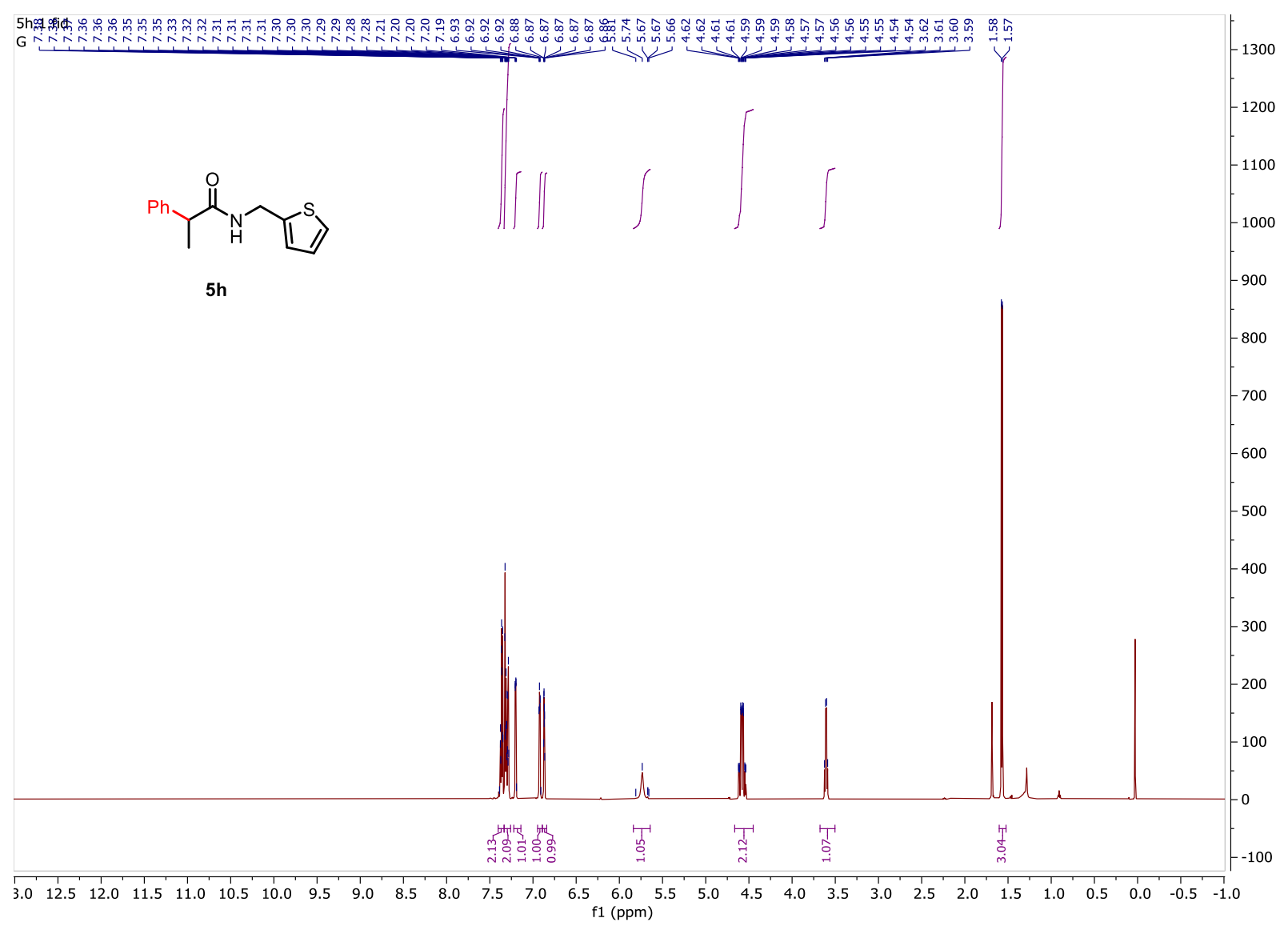




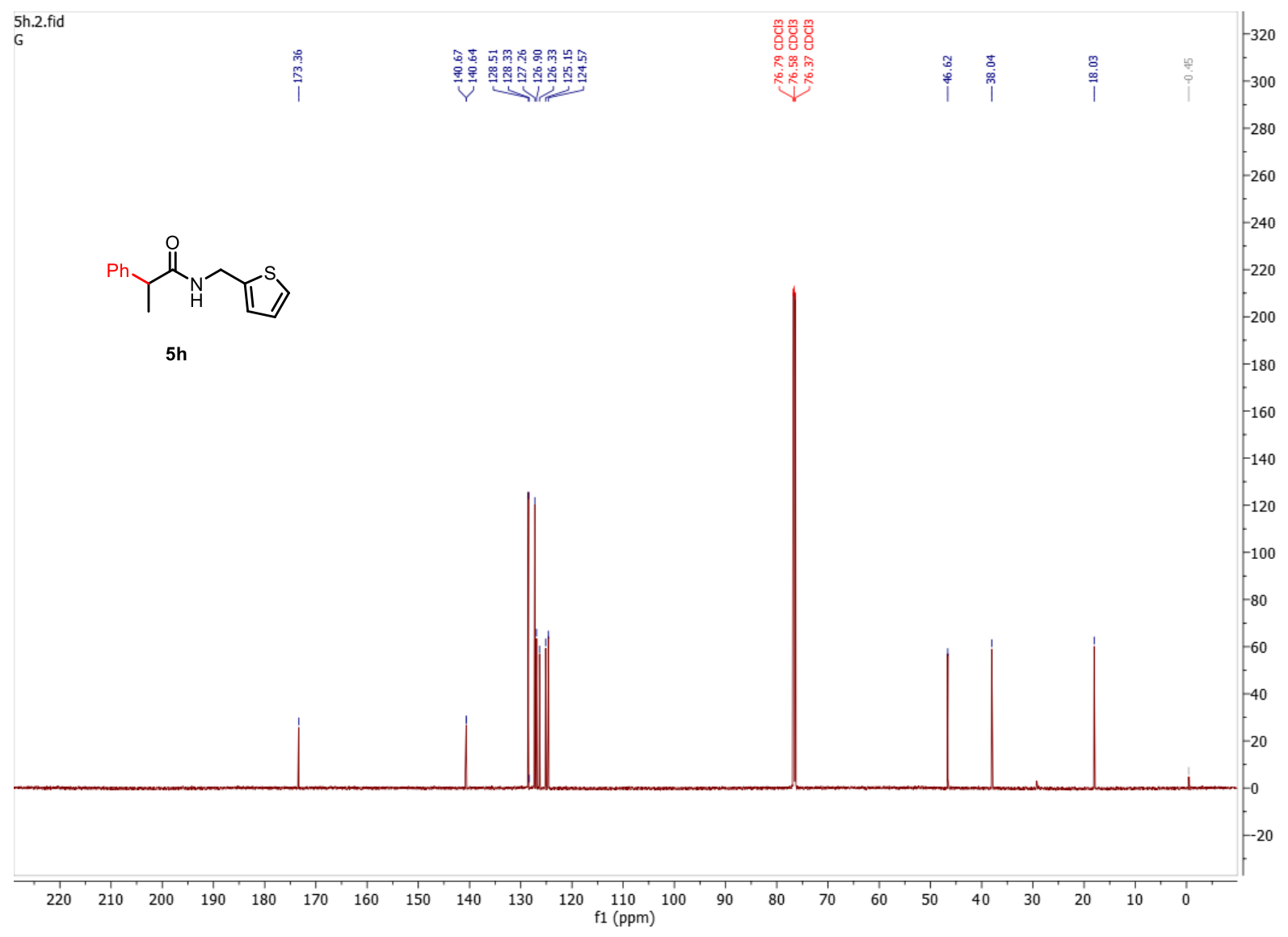




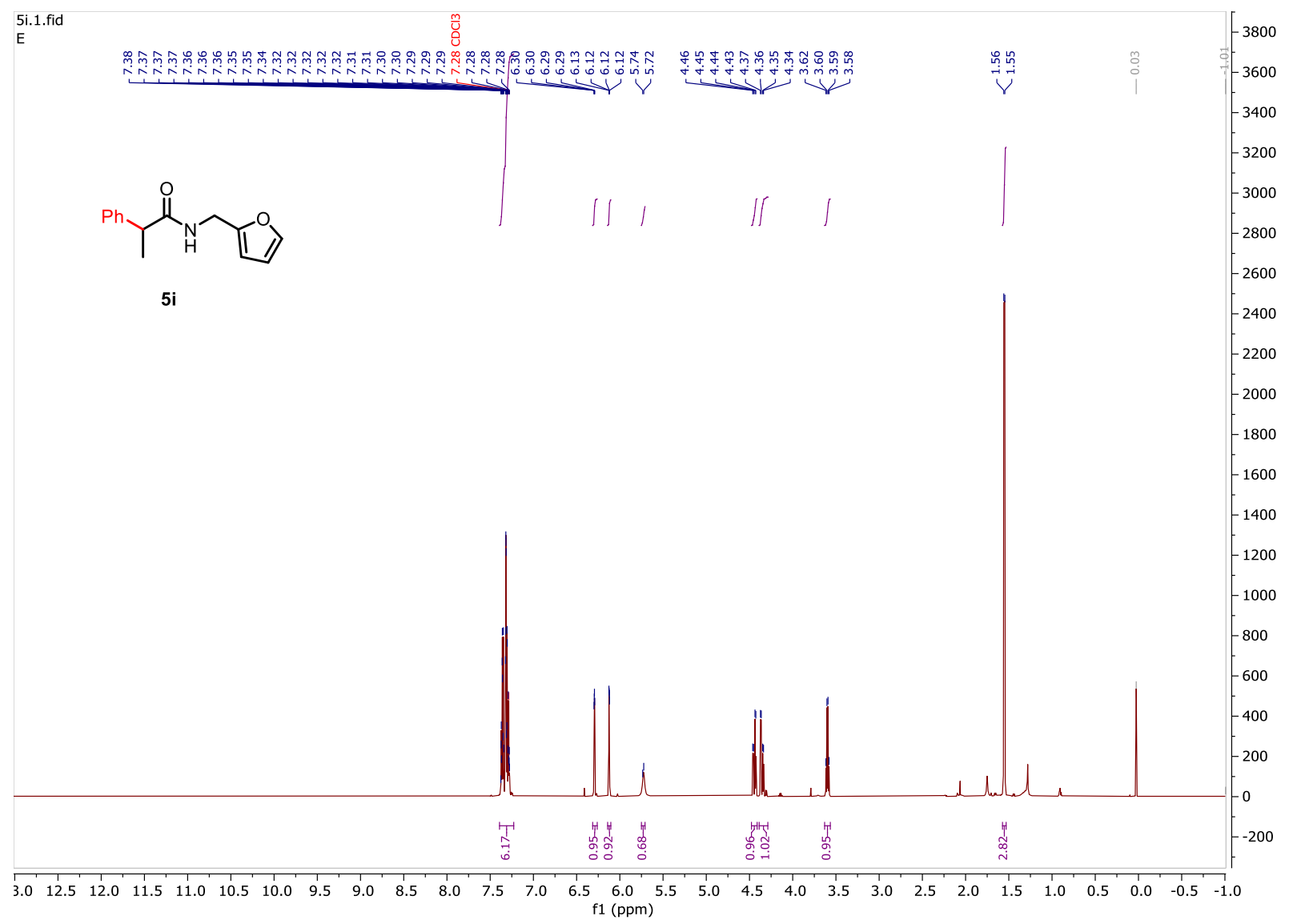




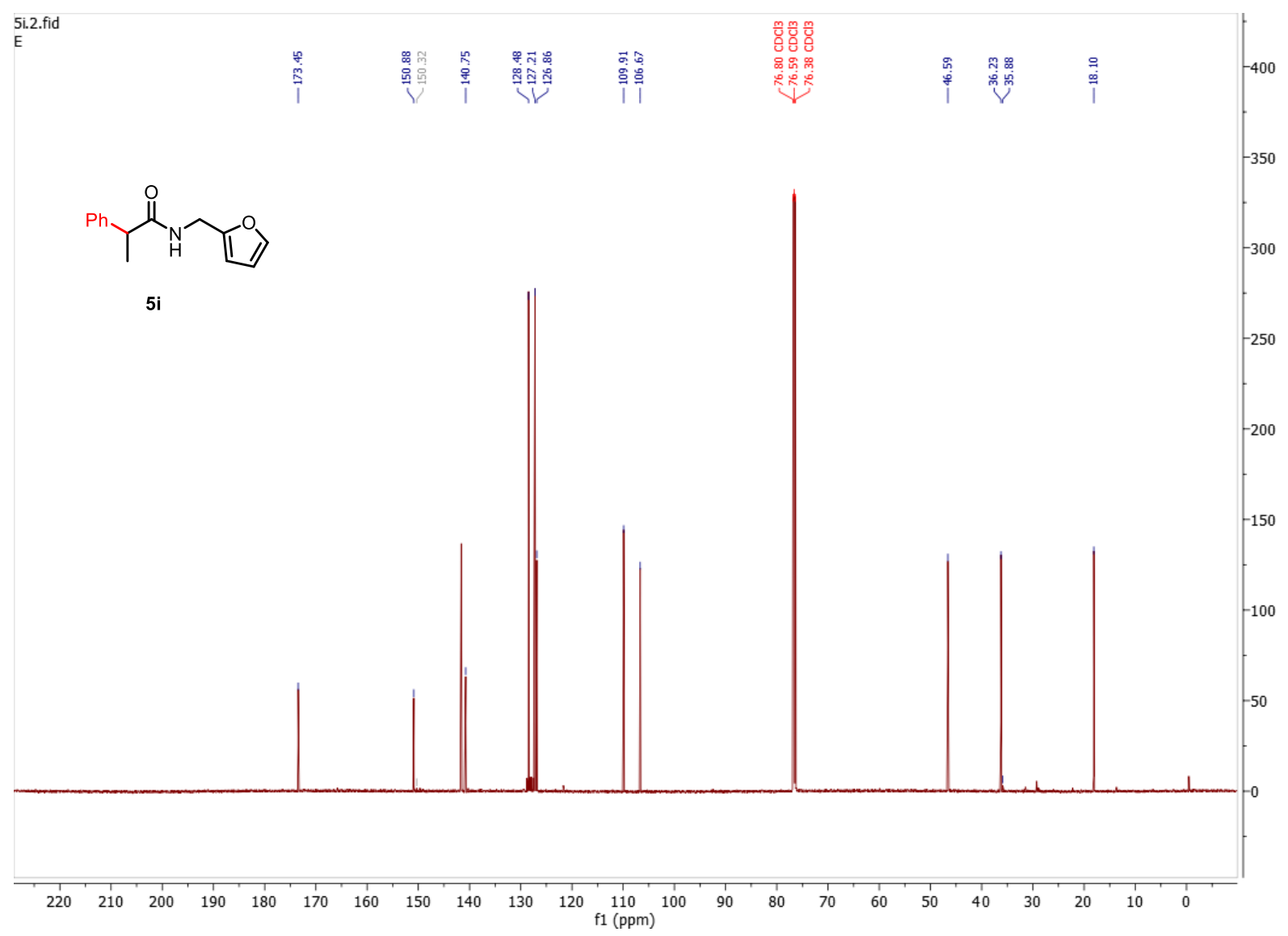




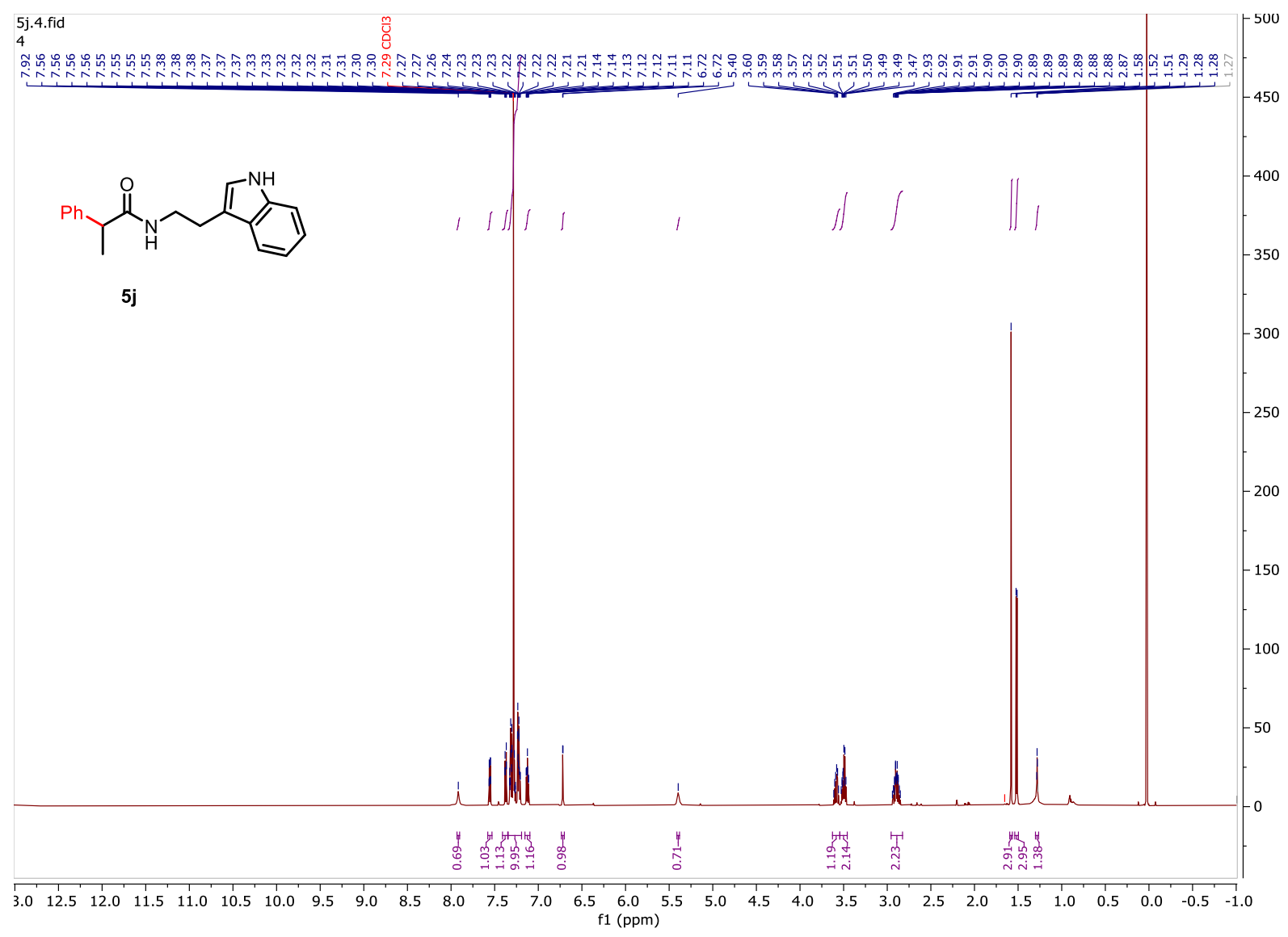




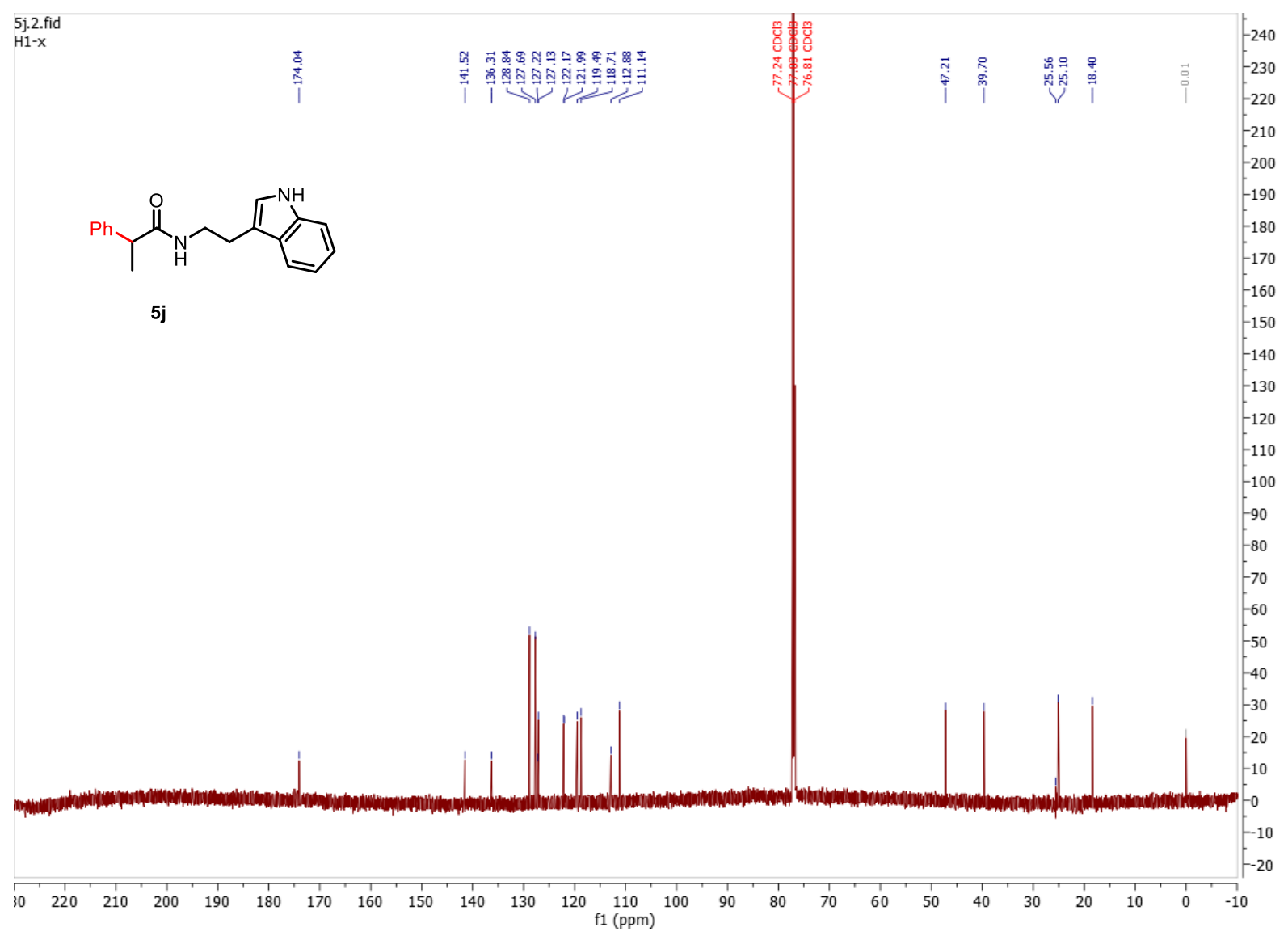




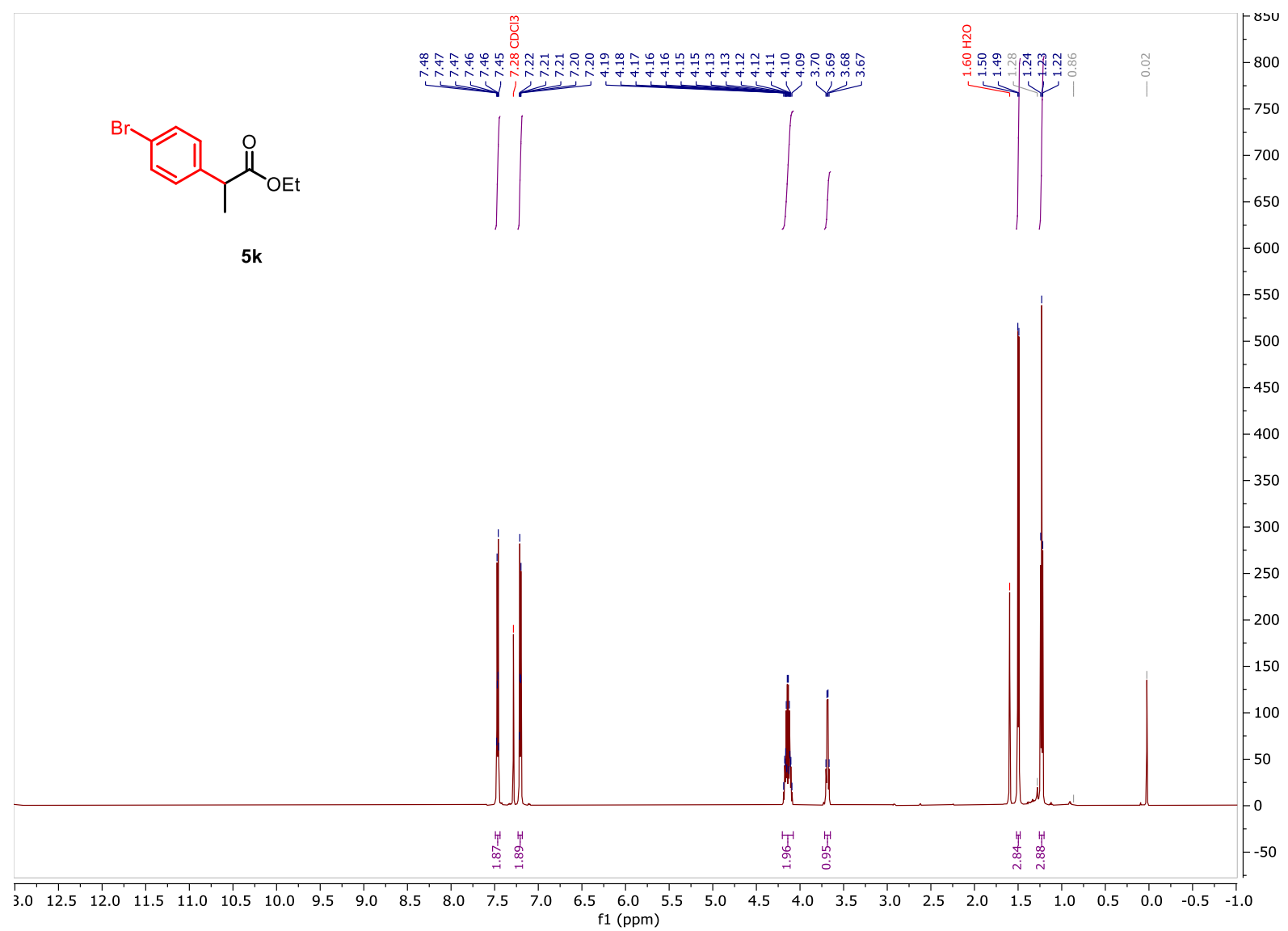




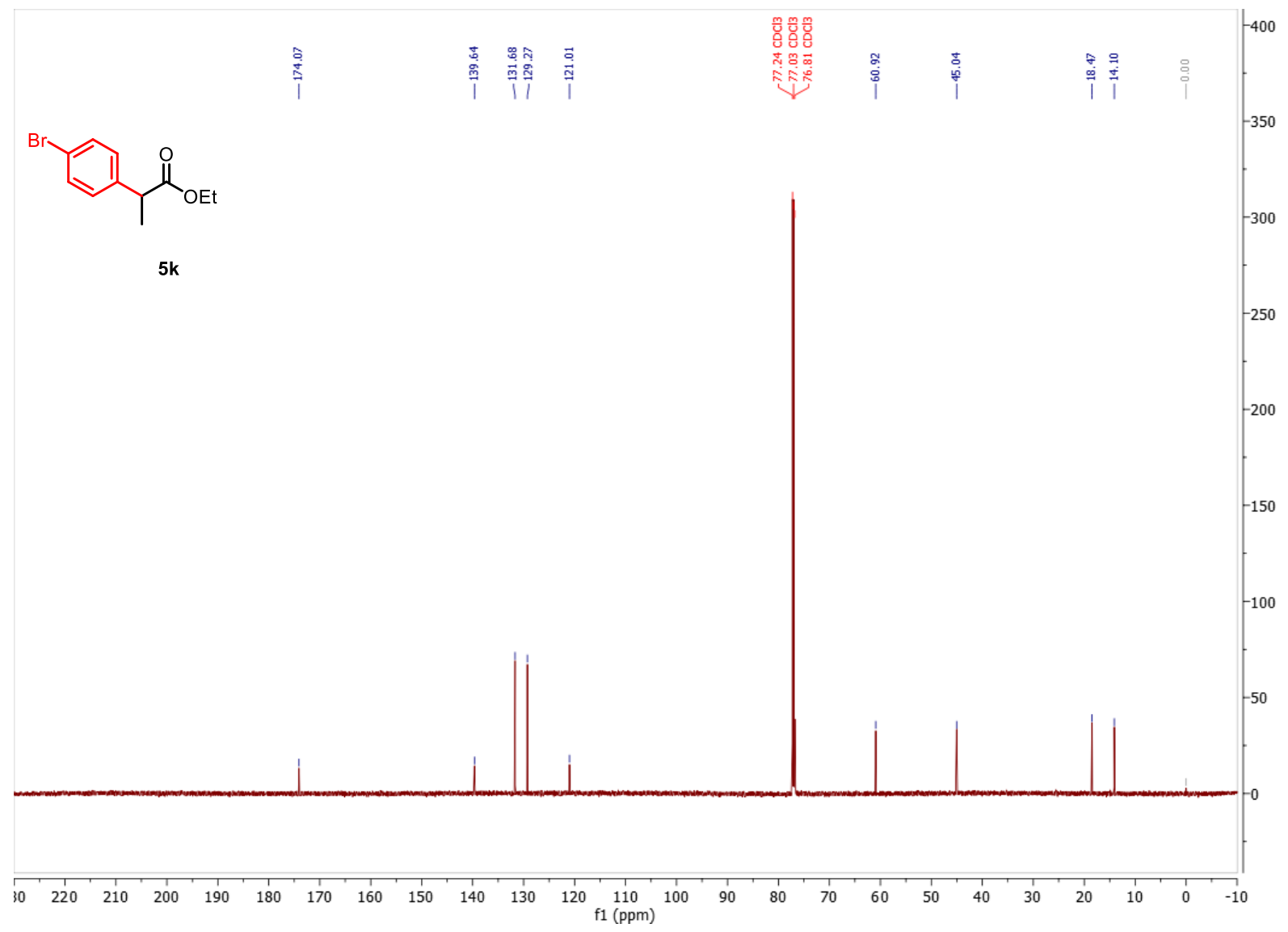

S113 


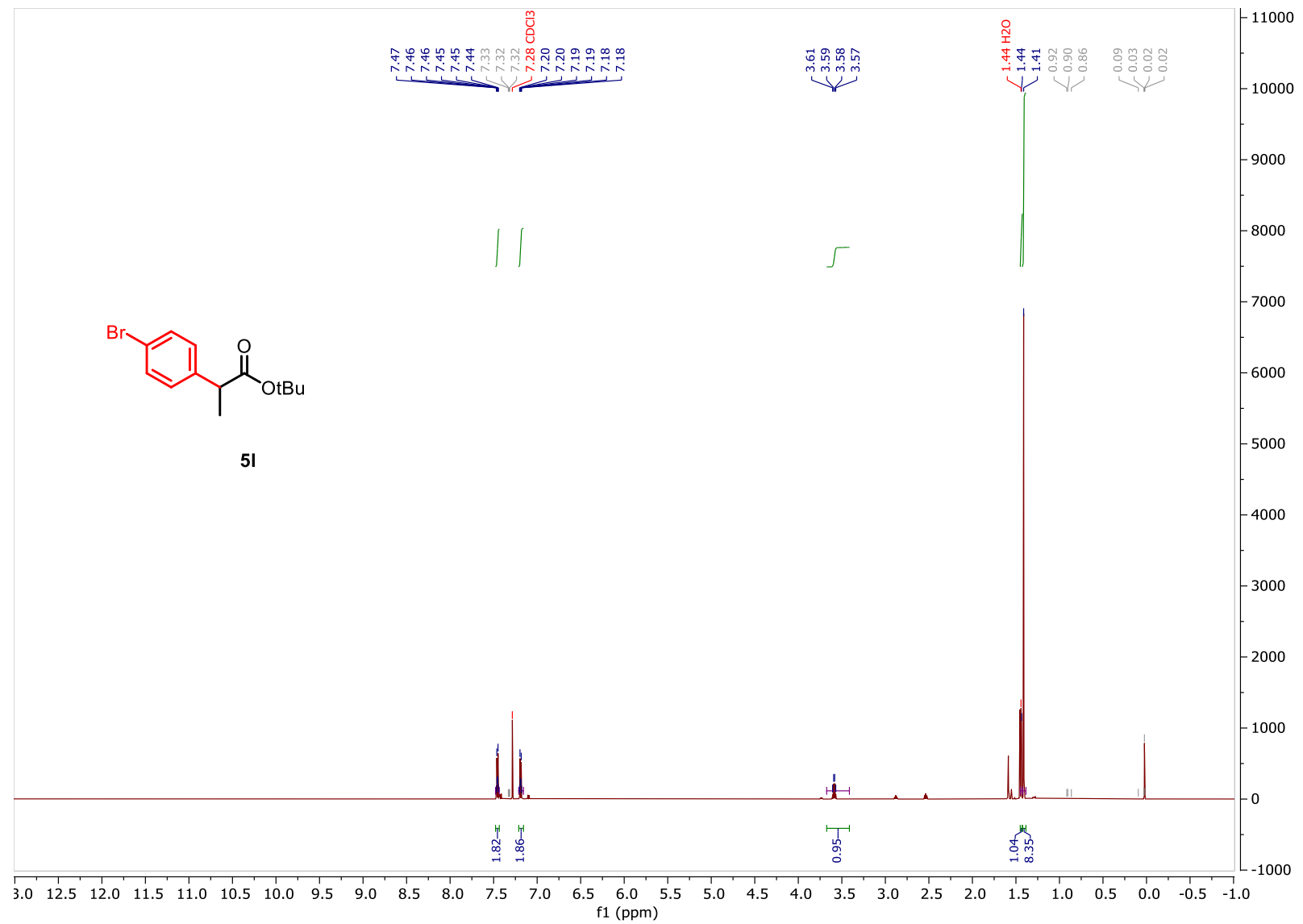




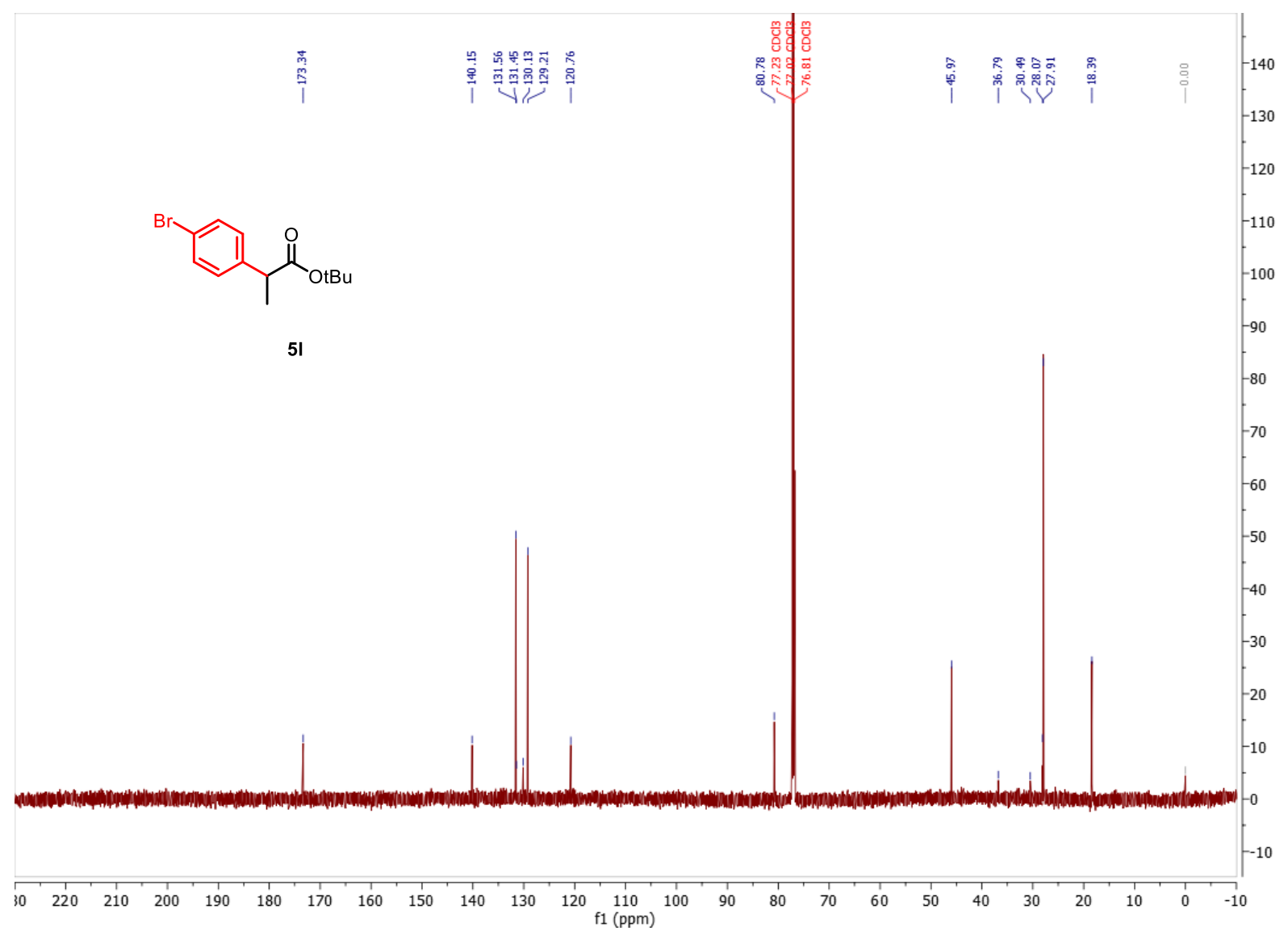

S115 


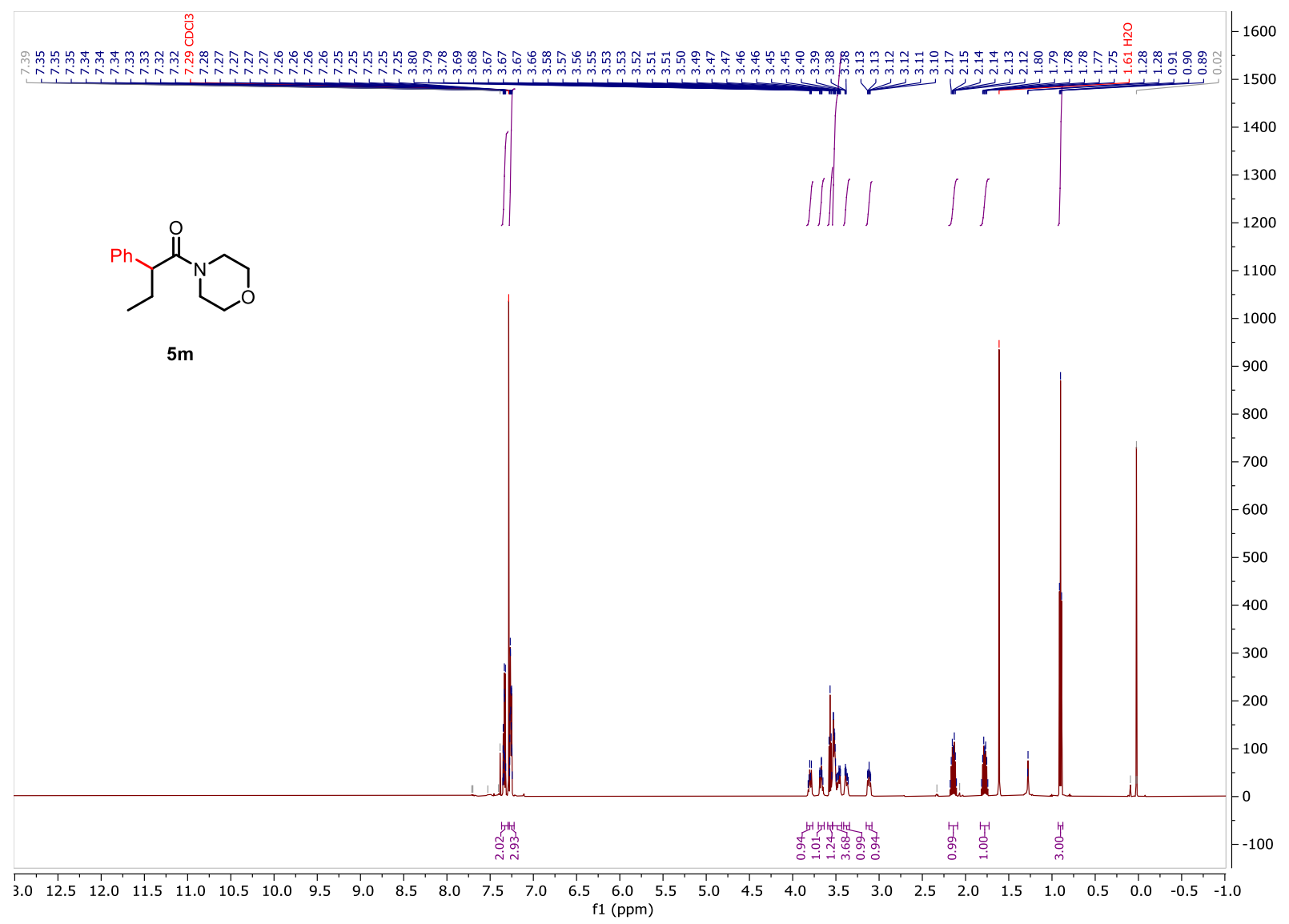




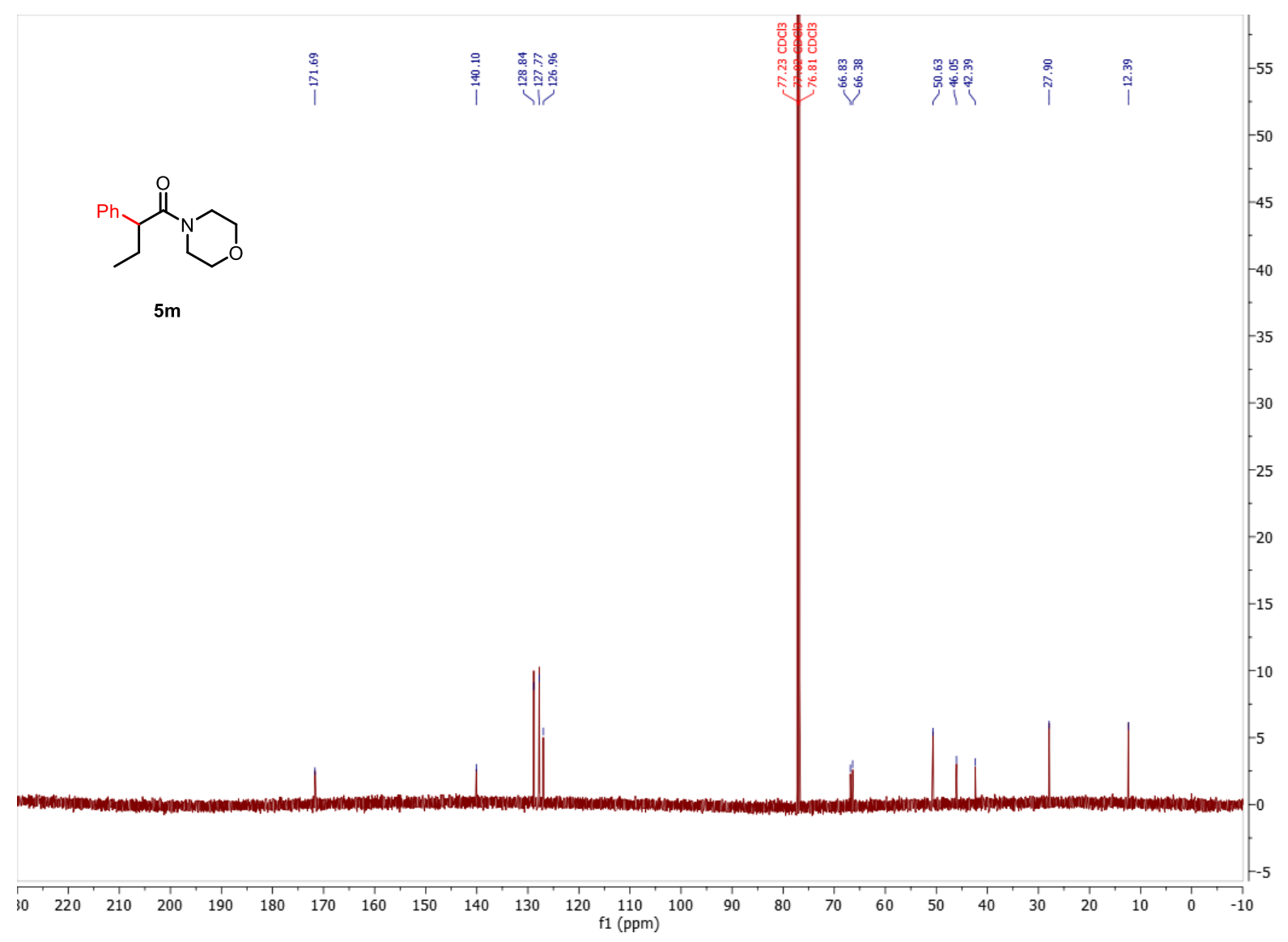




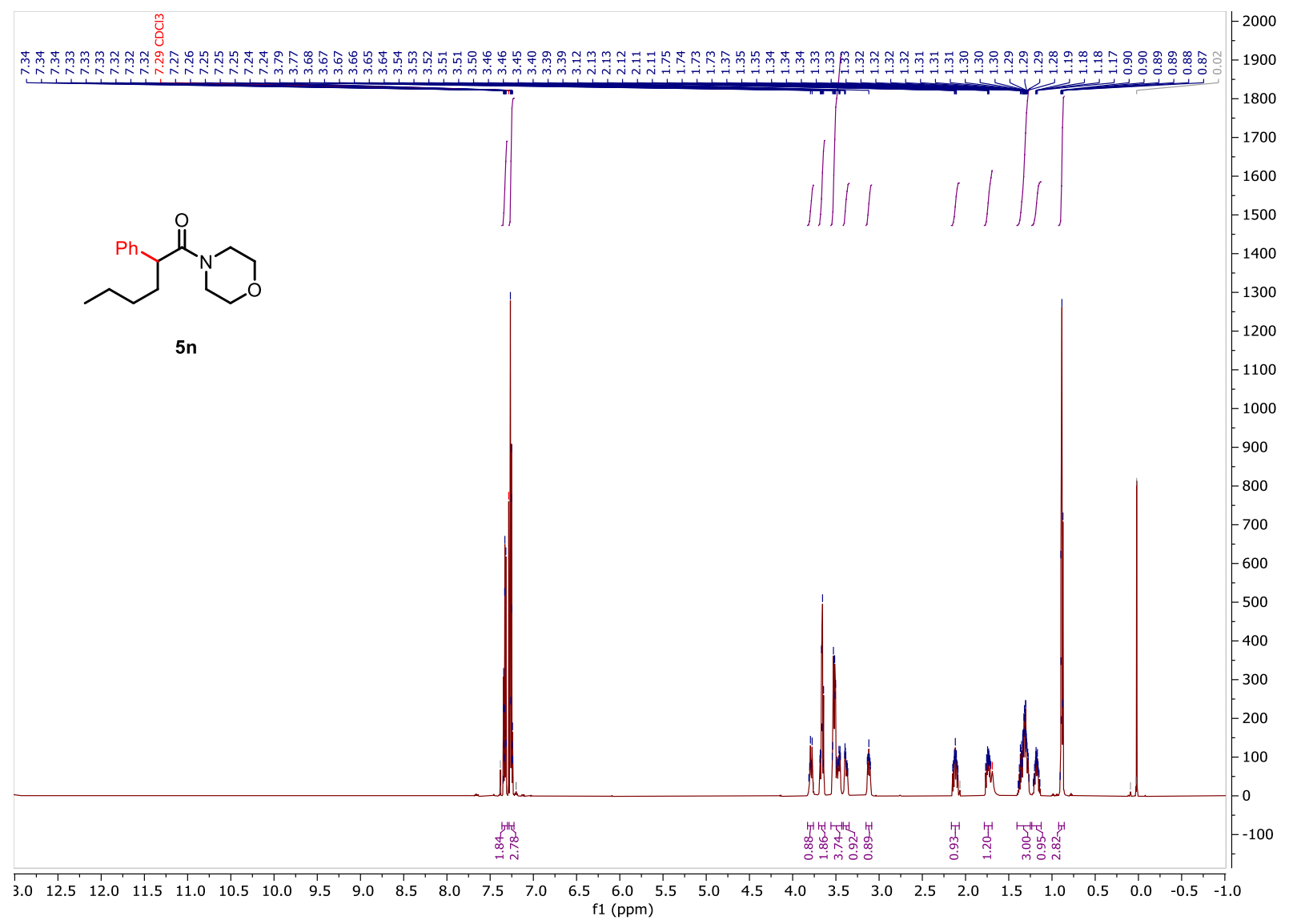




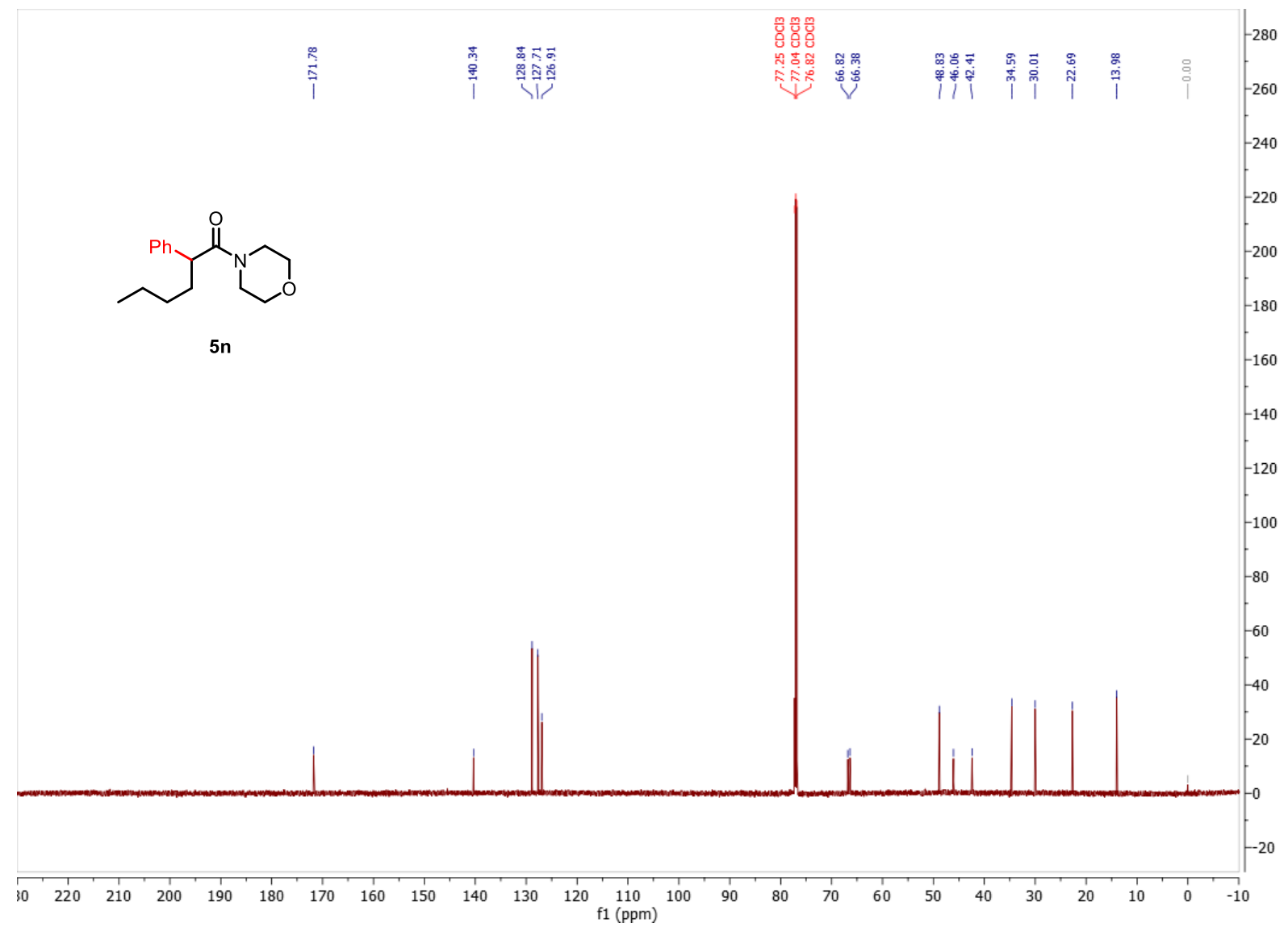




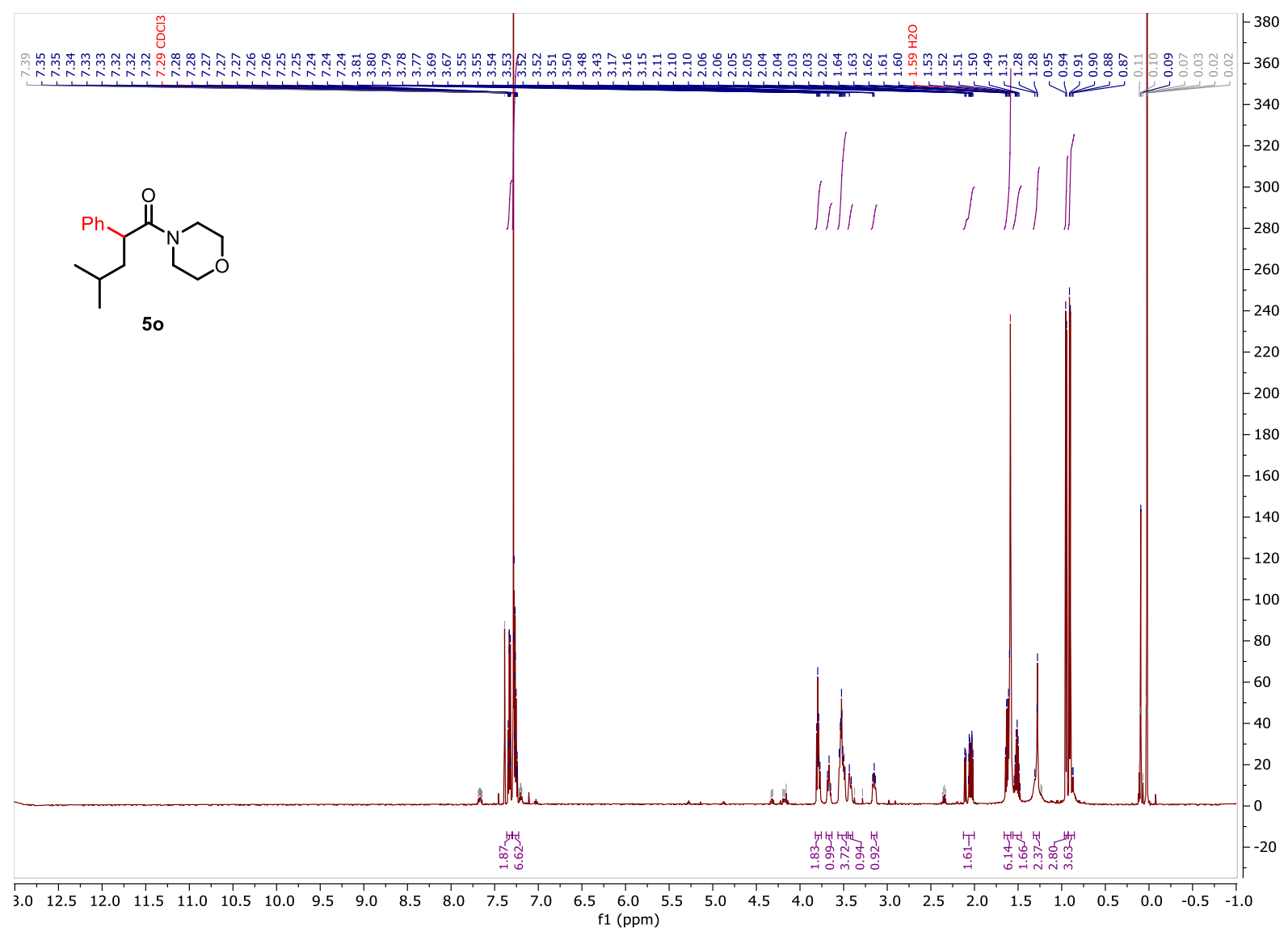




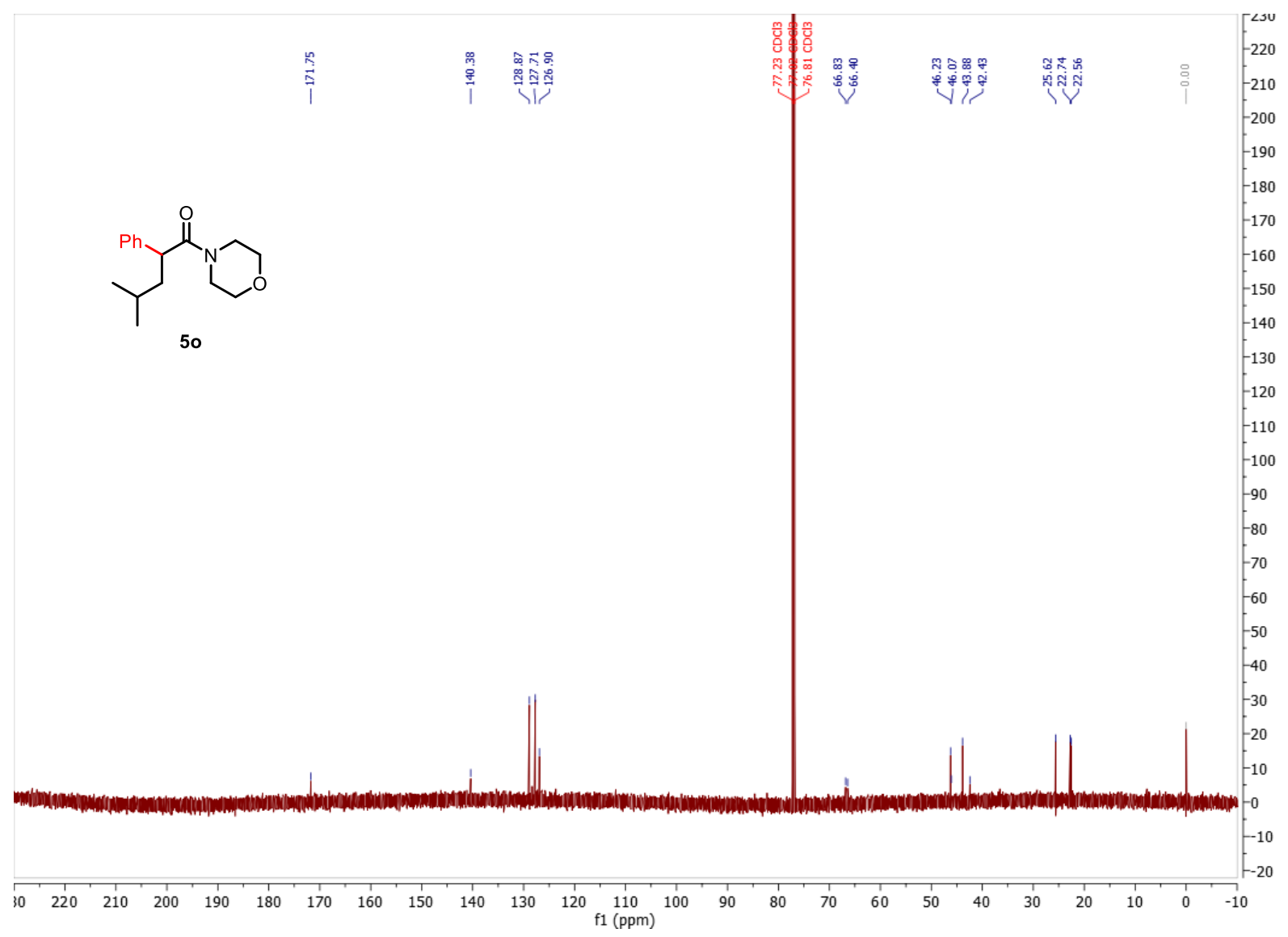




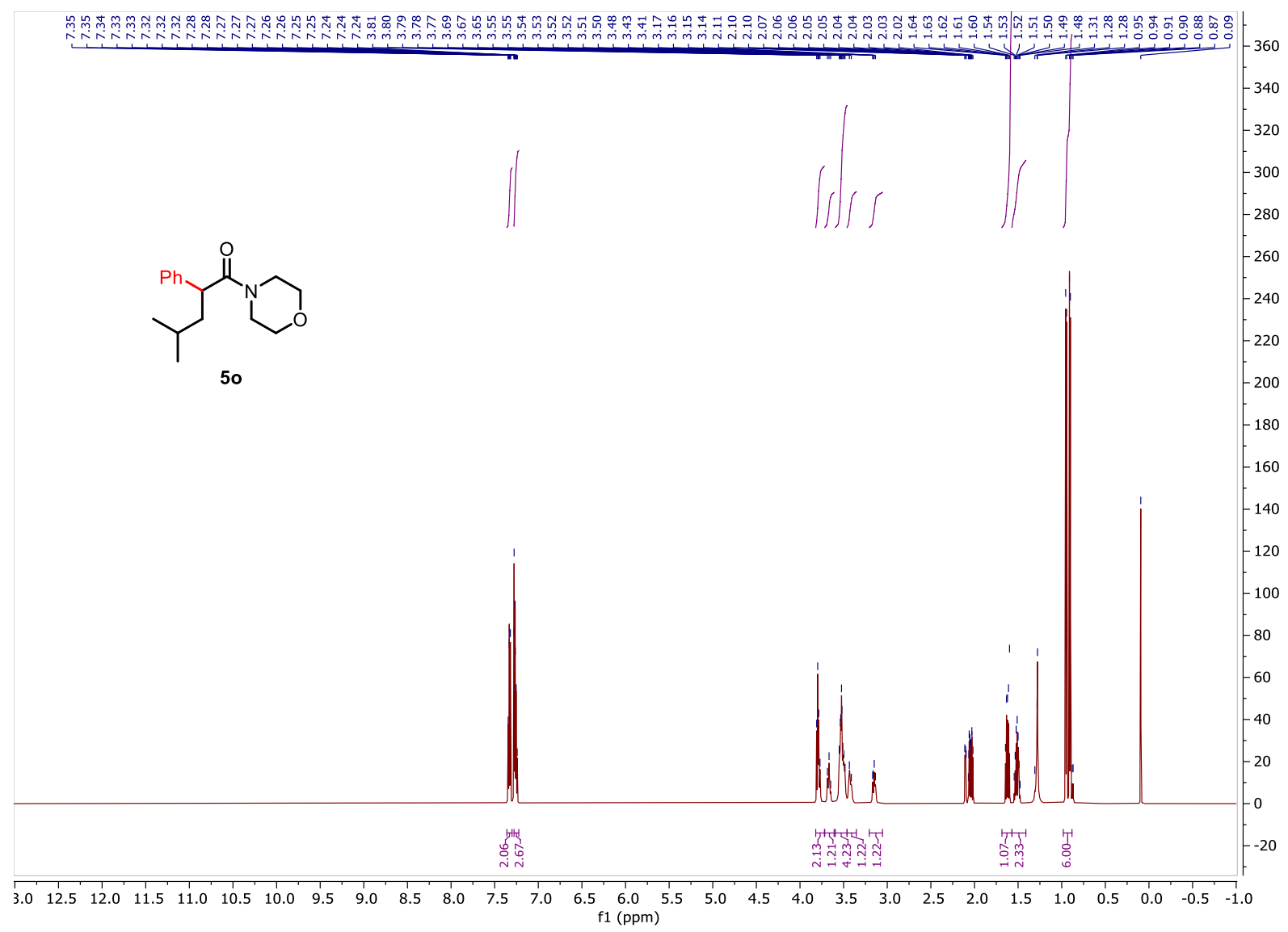




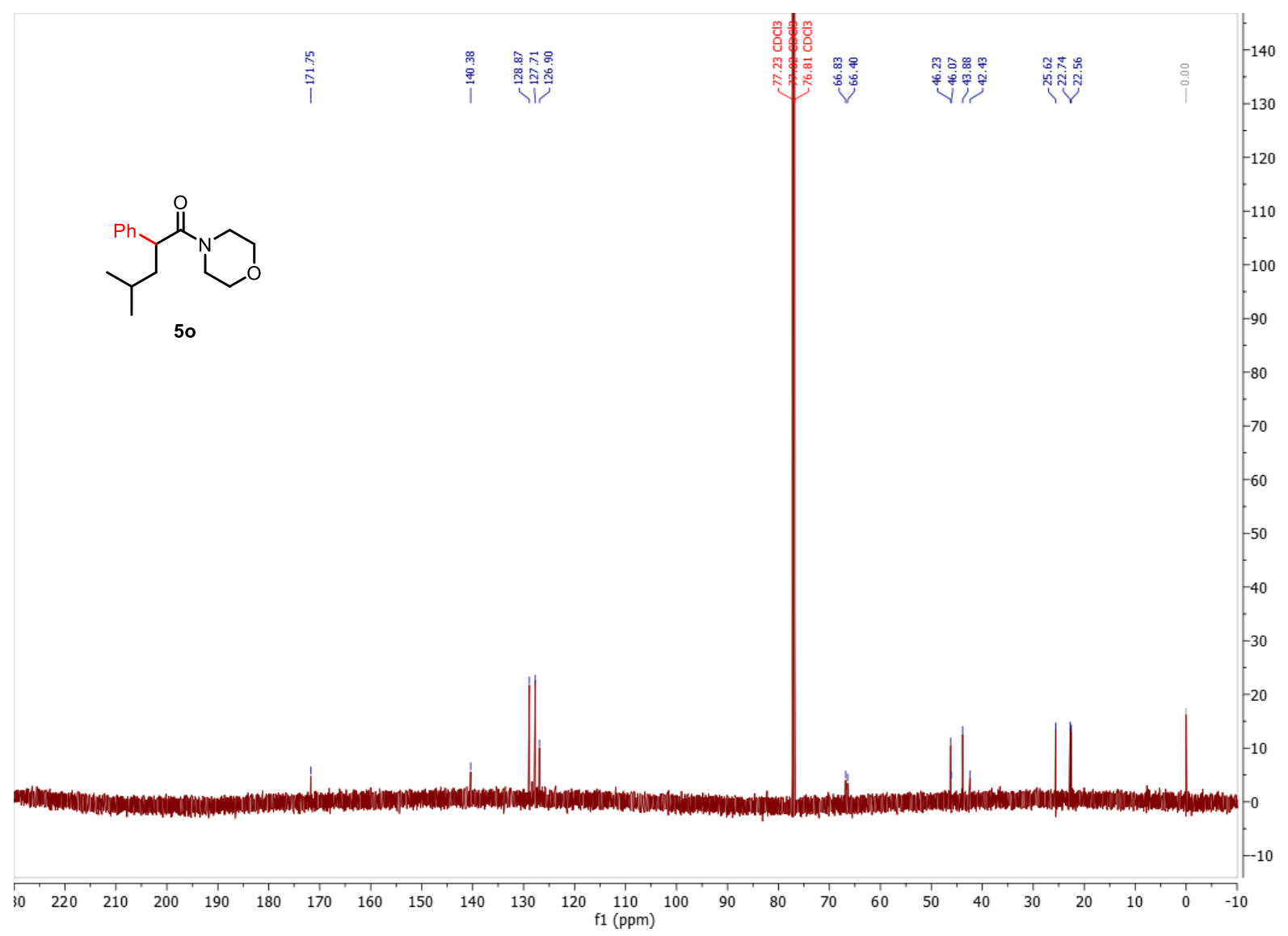




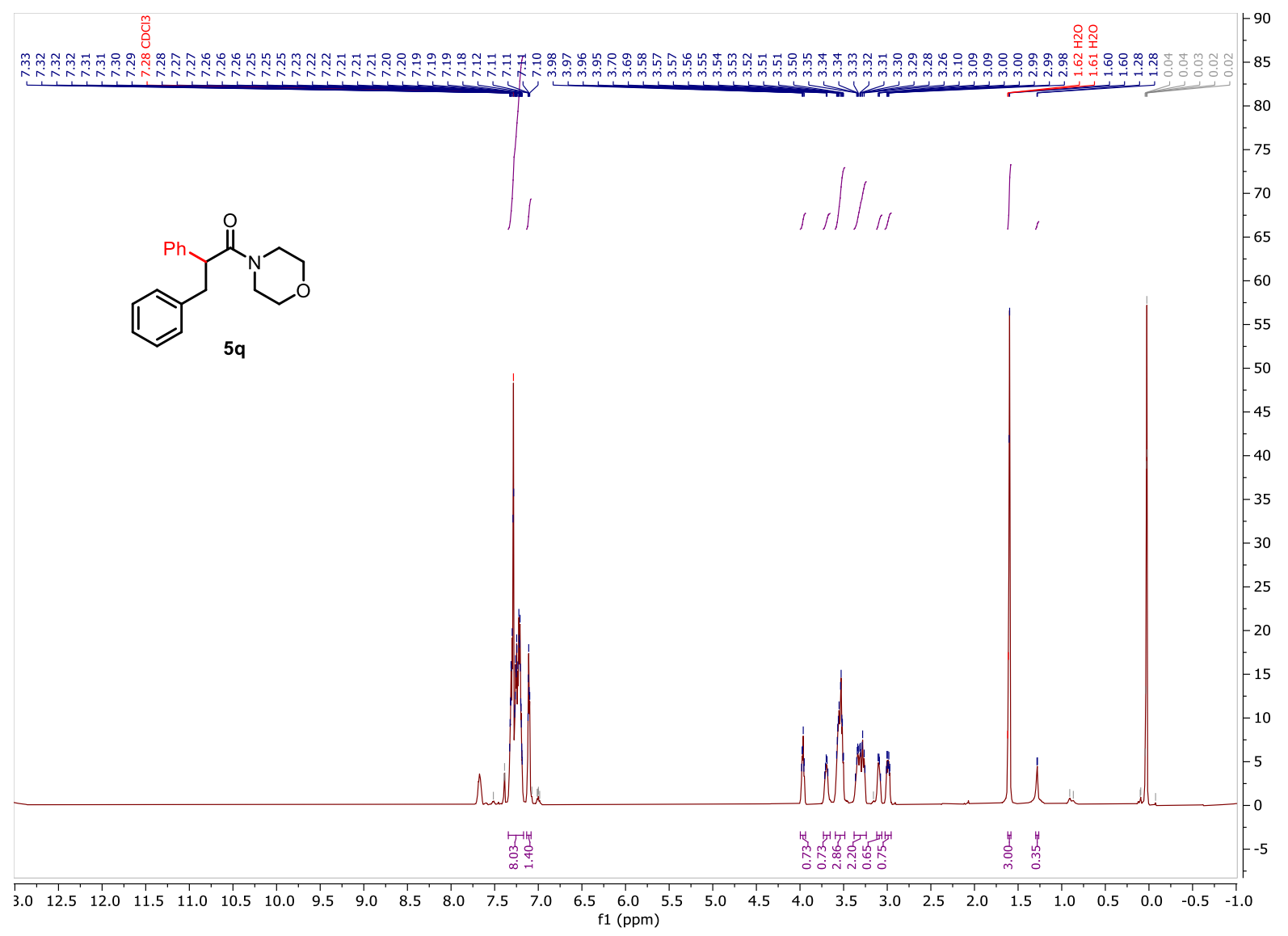




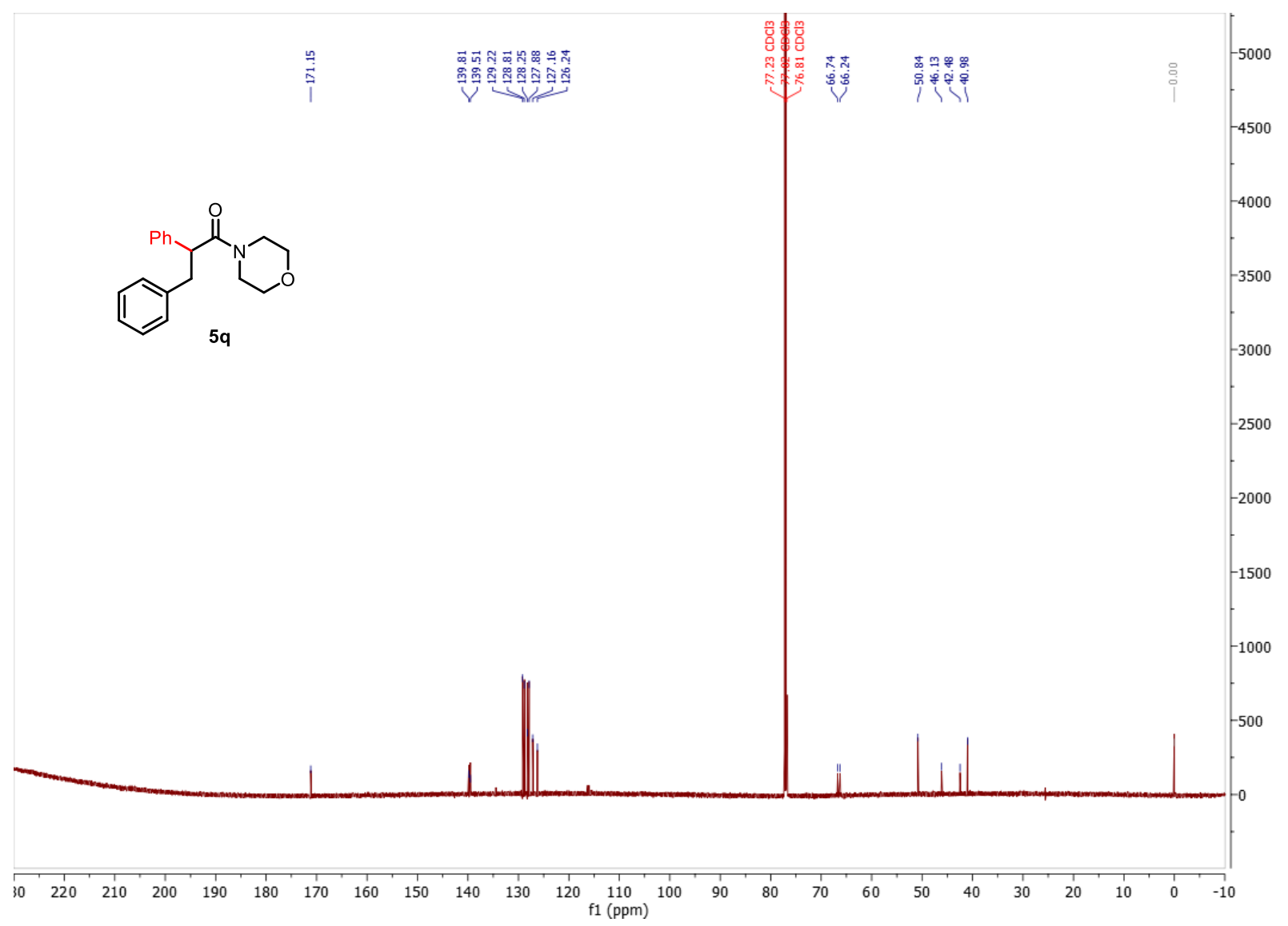




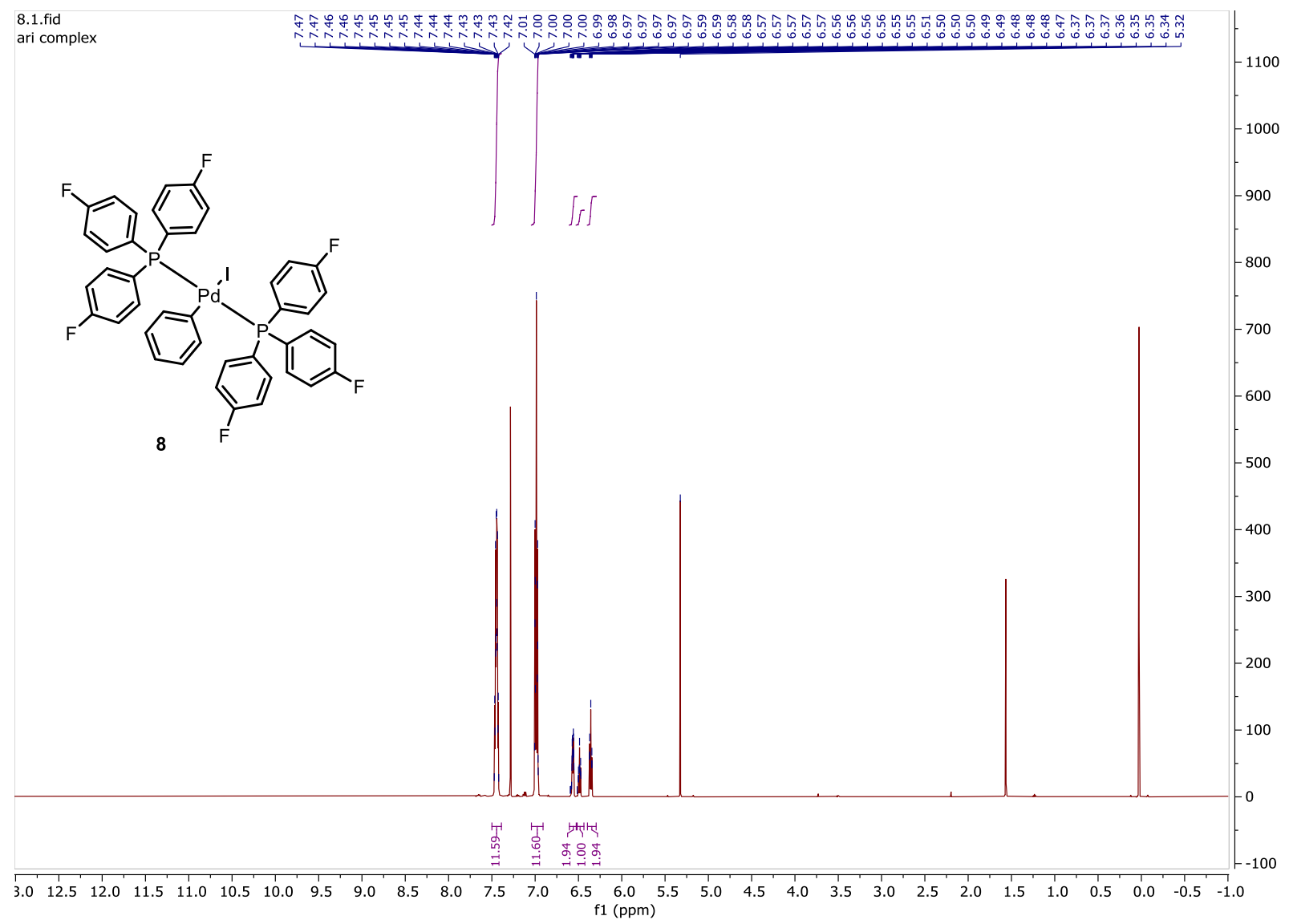




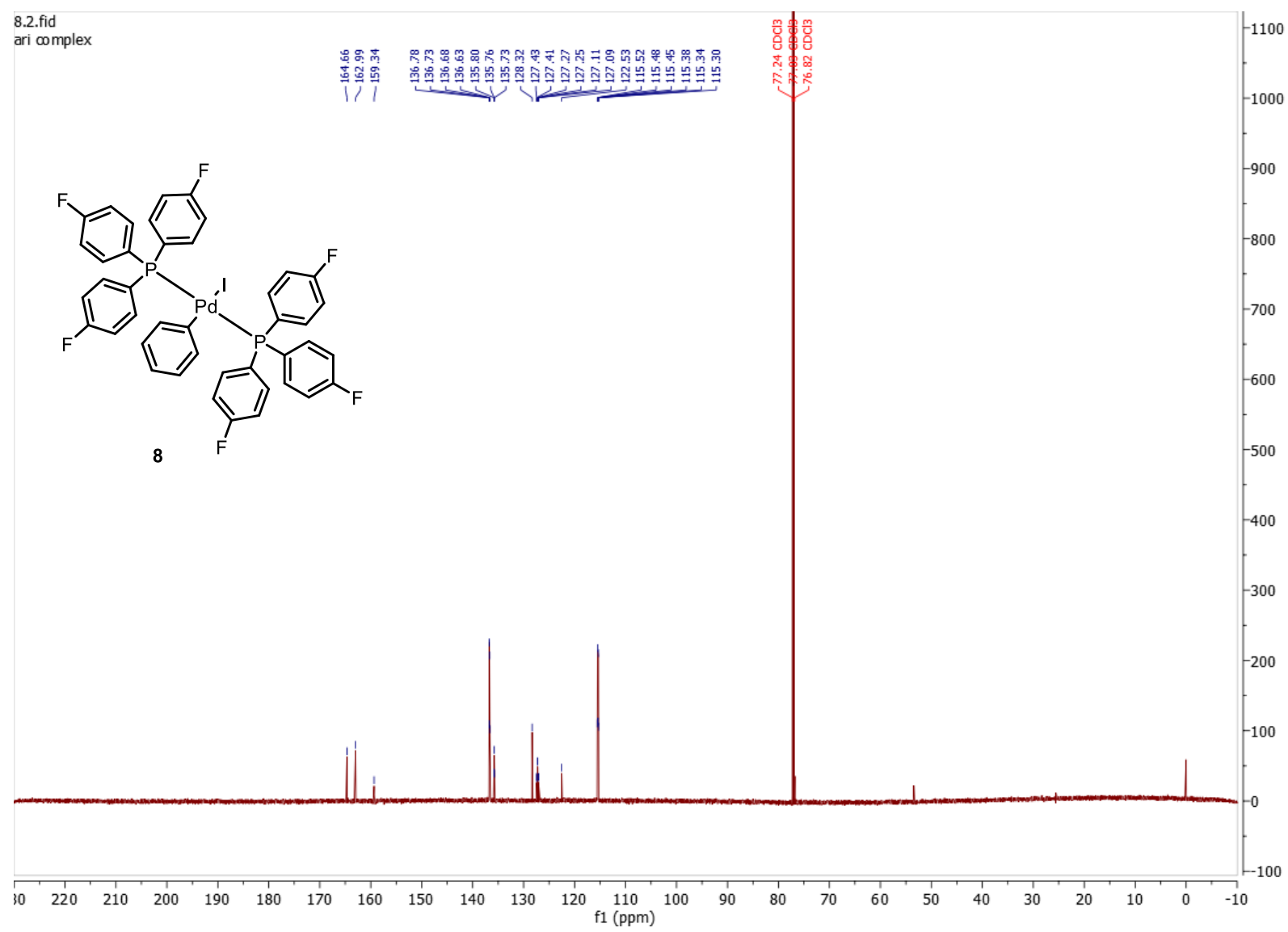




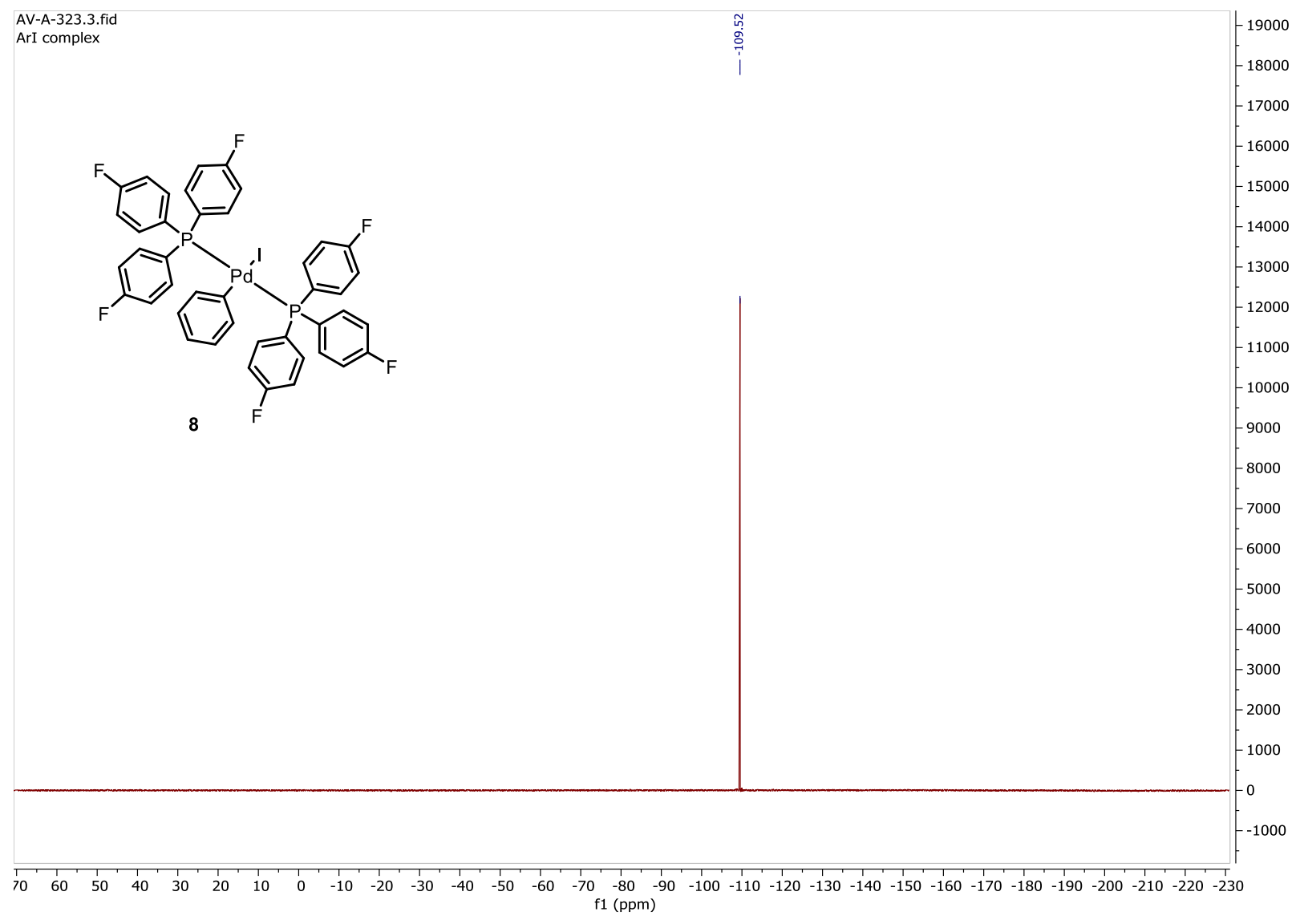

